UCRL-53635

Distribution Category UC-34A

UCRI, $=-53635$

DE85 014144

\title{
Spatial Distribution of Radiation from the \\ Beam Line VIII-W 15-Period Wiggler
}

\author{
E. M. Lent
}

W. C. Dickinson

Manuscript date: May 13, 1985

\section{LAWRENCE LIVERMORE NATIONAL LABORATORY \\ University of California - Livermore, California - 94550}

Available from: National Technical Information Service • U.S. Department of Commerce 5285 Port Royal Road • Springfield, VA 22161 • \$14.50 per copy • (Microfiche \$4.50)

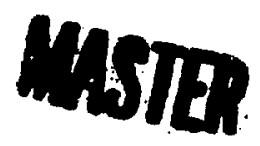


Abstract. . . . . . . . . . . . . . . . . . . . . 1

Introduction. . . . . . . . . . . . . . . . . . . . . 1

Discussion. . . . . . . . . . . . . . . . . . . . . 2

Explanation of Figures. . . . . . . . . . . . . . . . . . 4

References. .......................... 6

Appendix A - Illustration of Contributions of Smearing

Functions on Photon Spatial Distributions . . . . . . . . . . . 7

Appendix B - Computer-Generated Figures . . . . . . . . . . . . 16

Appendix C - Tables of Integrated Absolute Numbers of Photons . . . . 81 
We have written a computer program to calculate the spatial distribution of the radiation from insertion devices operating in nominal wiggler mode $(K=15.6)$, for which the incoherent emission dominates, and our asamption of negligible coherent emiasion is valid. This program has been applied to the 15-period wiggler now being deaigned by the Lawrence Berkeley Laboratory for Beam Line VIII-W on the Stanford Positron-Electron Accumulation Ring (SPEAR). An approximate spreading function has been applied at each photon energy to account for the intrinsic photon divergence. The effects of the finite wiggler length and the spatial and angular spread of the electron beam have been included.

Graphical plots are provided for three different electron energies, 1.8 $\mathrm{GeV}, 3.0 \mathrm{GeV}$, and $3.4 \mathrm{GeV}$, and for a range of photon energies. Separate plots are provided for total radiation, parallel polarization component, and perpendicular polarization component.

\section{INTRODUCTION}

The University of California/National Laboratory Team plans to install a 15-period wiggler as part of the Stanford Positron-Electron Accumlation Ring (SPEAR) when SPEAR shts down for the the summer of 1986. The wiggler, which is being designed and built by the Lawrence Berkeley Laboratory, has spatially periodic, high magnetic fields that confine the electron beam to a horizontal, sinusoidal path, thus producing copious amounts of radiation with no net horizontal displacement or deflection of the electron beam. To properly design experimental equipment for the wiggler beam line (Beam Line VIII-W), it is desirable to accurately characterize the spatial distribution of the wiggler's radiation as a function of photon energy. 
Schwinger[1] presents an expression for the instantaneous power radiated in a narrow energy band by a monoenergetic electron in circular orbit. This expression can be used in the following convenient, form:

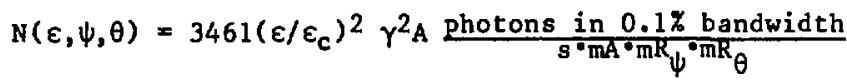

where $\psi$ is the angle between the direction of emission and the instantaneous orbital plane, and $\theta$ is the angle, projected on the orbital plane, between the direction of emission and direction of motion. The beam is characterized

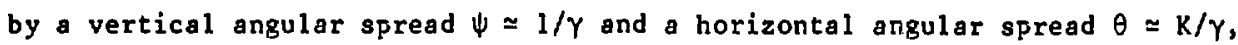
where

$\gamma=E / m_{0} c^{2}$

$\mathrm{K}=0.0934 \mathrm{~B} \lambda$,

$E$ is the electron energy in GeV, B is the magnetic field strength in $k G$, and $\lambda$ is the wiggler wavelength in $\mathrm{cm}$. The factor $A$ is given by

$A=\left(1+x^{2}\right)^{2}\left[k_{2 / 3}^{2}(\xi)+\frac{x^{2}}{1+x^{2}} k_{1 / 3}^{2}(\xi)\right]$,

where

$x=\gamma \psi$,

and

$\xi=\frac{\varepsilon}{2 \varepsilon_{c}}\left(1+X^{2}\right)^{3 / 2}$

The critical energy $\varepsilon_{c}$ is given by

$\varepsilon_{c}(k e v)=2.218 \mathrm{E}^{3} / \rho=0.06651 \mathrm{BE}^{2}$, 
where $\rho$ is radius of curvature in $m$. The factors $k_{1 / 3}$ and $k_{2 / 3}$ are modified Bessel functions of the second kind, for which a tabular listing of values is given in Chapter 2 of [2]. The $K_{2 / 3}$ term in the expression for $A$ represents radiation polarized with the electric vector parallel to the orbital plane, whereas the $k_{1 / 3}$ term represents radiation polarized with the electric vector perpendicular to the orbital plane. Note that in the orbital plane $(\psi=0)$, the radiation is $100 \%$ horizontally polarized.

Eq. (1) is to be applied over the s: soidal path of the electron as it traverses the wiggler. For a given photon energy, this is done by dividing a single quadrant of a wiggler wavelength $\lambda$ into a number of intervals of equal length. Each interval is characterized by average values of $p_{,} E_{c}$, and $\theta$. The total radiation at angle $\theta$ is then obtained by multiplying by twice the number of wiggler perioda.*

It is convenient to expreas Eq. (1) in terms of a spatial distribution of radiation in the $x-y$ plane at a distance $R$ from the wiggler source. Hence,

$N(\varepsilon, x, y, R)=3461\left(\varepsilon / \varepsilon_{c}\right)^{2} \frac{Y^{2} A}{R^{2}} \frac{\text { photons in } 0.1 \% \text { bandwidth }}{s^{*} \mathrm{~mA}^{\cdot} \mathrm{mm}_{\mathrm{x}}{ }^{\cdot} \mathrm{mm}_{\mathrm{y}}}$,

where $R$ is in $m$. If the total length of the wiggler is much less than $R$, all radiation can be assumed (to good approximation) to originate from the center point of the wiggler length. However, our wiggler will be $2 \mathrm{~m}$ long, and the $x-y$ plane of interest is $8.5 \mathrm{~m}$ from the wiggler center (the position of the first reflecting mirror). Therefore, we must take into account the finite photon source length by applying Eq. (8) to several points along the wiggler length and appropriately summing the results.

Eq. (1) applies only to electrons traveling in circular orbit. For the sinus udal path of an electron in our wiggler, a horizontal spreading function must be applied to Eq. (1). This is approximated by a Gaussian distribution with standard deviaton at low photon energy $[3]\left(\varepsilon \ll \varepsilon_{c}\right)$

$\sigma=\frac{1}{\gamma}\left(\varepsilon_{c} / \varepsilon\right)^{1 / 3}$

and for high photon energy $\left(\varepsilon>\varepsilon_{c}\right)$,

* Two quadrants in each wiggler period contribute radiation to one side of the wiggler axis, and the other two quadrants to the other side. For our wiggler, since the horizontal excursion of the electron from the wiggler axis is only about $0.05 \mathrm{~mm}$, this spreading effect can be neglected. 
$\sigma=\frac{1}{\gamma}\left(\varepsilon_{c} / 3 \varepsilon\right)^{1 / 2}$

A suitably smooth transition is made between these two functions over our range of photon energies. Although the above widths are valid only for unpolarized radiation, this same spreading was also applied to the horizontal and vertical components. A more exact treatment is beyond the scope of this work.

Also, a "smearing" function must be introduced to take into account the spatial and angular smearing of the electron beam in the storage ring. For the SPEAR, this smearing can be characterized by the following set of vertical and horizontal standard deviations:

$$
\begin{array}{ll}
\sigma_{\mathrm{H}} \simeq 2.5 \mathrm{~mm} & \sigma_{\mathrm{V}} \simeq 0.15 \mathrm{~mm} \\
\sigma_{\mathrm{H}}^{\prime} \simeq 0.18 \mathrm{mR} & \sigma_{\mathrm{V}}^{\prime} \simeq 0.03 \mathrm{mR}
\end{array}
$$

At the distance $R$ from the photon source, these angular and spatial spreads can be combined into a single horizontal and a single vertical standard deviation. Therefore, for $R=8.5 \mathrm{~m}, \sigma_{H}$ (TOTAL) $=2.9 \mathrm{~mm}$ and $\sigma_{\mathrm{V}}($ TOTAL $)=0.3 \mathrm{~mm}$.

This smearing is accomplished by first doing a one-dimensional convolution in the horizontal $(x)$ direction for each vertical $(y)$ displacement and then convolving in the vertical direction for each horizontal displacement. In each case, the Gaussian of appropriate width is used in the convolution.

\section{EXPLANATION OF FIGURES}

In Appendix A, we show, as a matter of interest, the individual contributions of each of these smearing effects on the final photon spatial distributions. We conclude that for low photon energies $\left(\varepsilon \ll \varepsilon_{c}\right)$, the final photon spatial distributions are determined almost entirely by the intrinsic spreading of the photon beam, whereas at high photon energies $\left(\varepsilon \geq \varepsilon_{c}\right)$, the effect of the electron beam spatial and angular spread on the final photon distributions, although not terribly important, is still not negligible. 
In Appendix B, one set of computer-generated figurea is provided for each of the following three electron energies: $1.80 \mathrm{GeV}, 3.00 \mathrm{GeV}$, and $3.40 \mathrm{GeV}$. It should be noted that all the plots include the three swearing effect: described above: 1) the intrinsic horizontal spread of the photon beam, 2) the effect of finite wiggler length, and 3 ) the effect of the assumed spatial and angular apread of the electron beam in the SPEAR. Each figure is an isometric plot of photon intenaity, in a single quadrant, for the listed photon energy at $8.50 \mathrm{~m}$ from the center of the wiggler. For each electron energy, plots are provided for the following photon energies: $20 \mathrm{eV}, 100 \mathrm{eV}, 500 \mathrm{eV}, 1 \mathrm{keV}, \varepsilon_{\mathrm{c}} / 2$, $\varepsilon_{c}$, and $5 \varepsilon_{c}$. The wiggler wavelength $\left(\lambda_{w}\right)$ is $12.85 \mathrm{~cm}$, and the maximum wiggler magnetic field $\left(B_{0}\right)$ is $13.0 \mathrm{kG}$. The critical energy, $\varepsilon_{c}$, is given for the point along the electron's sinusoidal path with the smallest radius of curvature.

Plots are provided for total intensity, intensity of the parallel polarization component, and intensity of the perpendicular polarization component. For the total and parallel polarization-intensity plots, the absolute intensity at the center of the photon distribution $(x=0, y=0)$ is 1 isted in the lower left-hand corner of each plot. For the perpendicular polarization plots, the listed absolute intensity is for the point of maximum intensity along the $y$ axis $(x=0)$. Intensity is given in the following units:

$$
\frac{\text { photons in } 0.1 \% \text { bandwidth at energy } \varepsilon}{s^{\circ} \mathrm{mA} \cdot \mathrm{mm}_{\mathrm{x}} \mathrm{mm}_{\mathrm{y}}}
$$

Each isointensity curve in a plot represents a fraction (listed in the table in the upper right-hand corner) of the listed absolute intensity. Two sets of intensity curves are shown in each plot. For the total and parallel polarization-intensity plots, these curves represent photon intensity along the $x$ axis at $y=0$ and along the $y$ axis at $x=0$. For the perpendicular polarization intensity plots, these curves represent photon intensity along the $y$ axis at $x=0$ and along the $x$ axis at the value of $y$ for which the absolute intensity is given. In Appendix C, we provide, for each of the above plots, able listing the integrated absolute number of photons (per second, per $\mathrm{mA}$ in $0.1 \%$ bandwidth) in bins that are $2 \mathrm{~mm}$ high by 5 or $10 \mathrm{~mm}$ wide. 


\section{REFERENCES}

1. J. Schwinger, "On the Classical Radiation of Accelerated Electrons," Phys. Rev. 75, 1912 (1949).

2. H. Winick and S. Doniach (Eds.), Synchrotron Radiation Research, Plenum Press, New York, (1980).

3. J.D. Jackson, Classical Electrodynamics, Wiley Sons, New York, (1962). 


\section{ILLUSTRATION OF CONTRIBUTIONS OF SMEARING FUNCTIONS}

ON PHOTON SPATIAL DISTRIBUTIONS

It is interesting to plot the photon spatial distributions as given by the Schwinger formula, Eq. (1), and then add the requisite smearing contributiona, one at a time, to observe the effect of each in determining the final spatial diatributions. The following figures show the results at one electron energy, $E=3 \mathrm{GeV}$, and two photon energies, $\varepsilon=20 \mathrm{eV}$ and $\varepsilon=\varepsilon_{c}=7.78 \mathrm{keV}$.

The first set of four figures is for $\varepsilon=20 \mathrm{eV}$. Figure $A 1$ is just a plot of Eq. (1). Note the very sharp cutoff at the outer edge of the distribution. In Fig. A2 is the distribution when the intrinsic horizontal spread of the photon beam is convoluted with Eq. (1). Although there is no effect on the intensity at the center $(x=0, y=0)$, there is a strong smearing out of the distribution at the outer edge. To this smeared distribution, Fig. A3 adds the finite wiggler length, which has almost no effect on the spatial distribution. Finally, Fig. A4 adds the convolution of the assumed spatial and angular spread of the electron beam in the storage ring. Again, there is almost no effect on the spatial distribution. Therefore, we conclude that at very low photon energiea, the final spatial distribution is almost completely governed by the intrinsic vertical and horizontal spreading of the photon beam.

The second set of four figures is for $\varepsilon=\varepsilon_{c}=7.78 \mathrm{keV}$. Figure A5 is again a plot of Eq. (1). Figure A6 convolutes the intrinsic horizontal spread of the photon beam. The effect is seen to be negligible. Figure A7 adds the finite wiggler length. The result is a small additional increase in the horizontal beam spread. Figure $A 8$ adds the effect of convoluting the assumed spatial and angular spread of the electron beam in the storage ring. This results in an additional small spreading of the photon distribution, both vertically and horizontally. We conclude that at higher energies, the only noticeable effect of the smearing functions on the final photon spatial distribution is that due to the spatial and angular spread of the electron beam, and even this is not very important. 
wiggler: 30 poles; $B=13.0 \mathrm{hG} ; 1$ ambda $=12.85 \mathrm{~cm}$

$8.5 \mathrm{~m}$ from center of wiggler to mirror

electron energy $=3.00$ e+00 GeV critical energy $=7.78 \mathrm{e}+00 \mathrm{heV}$
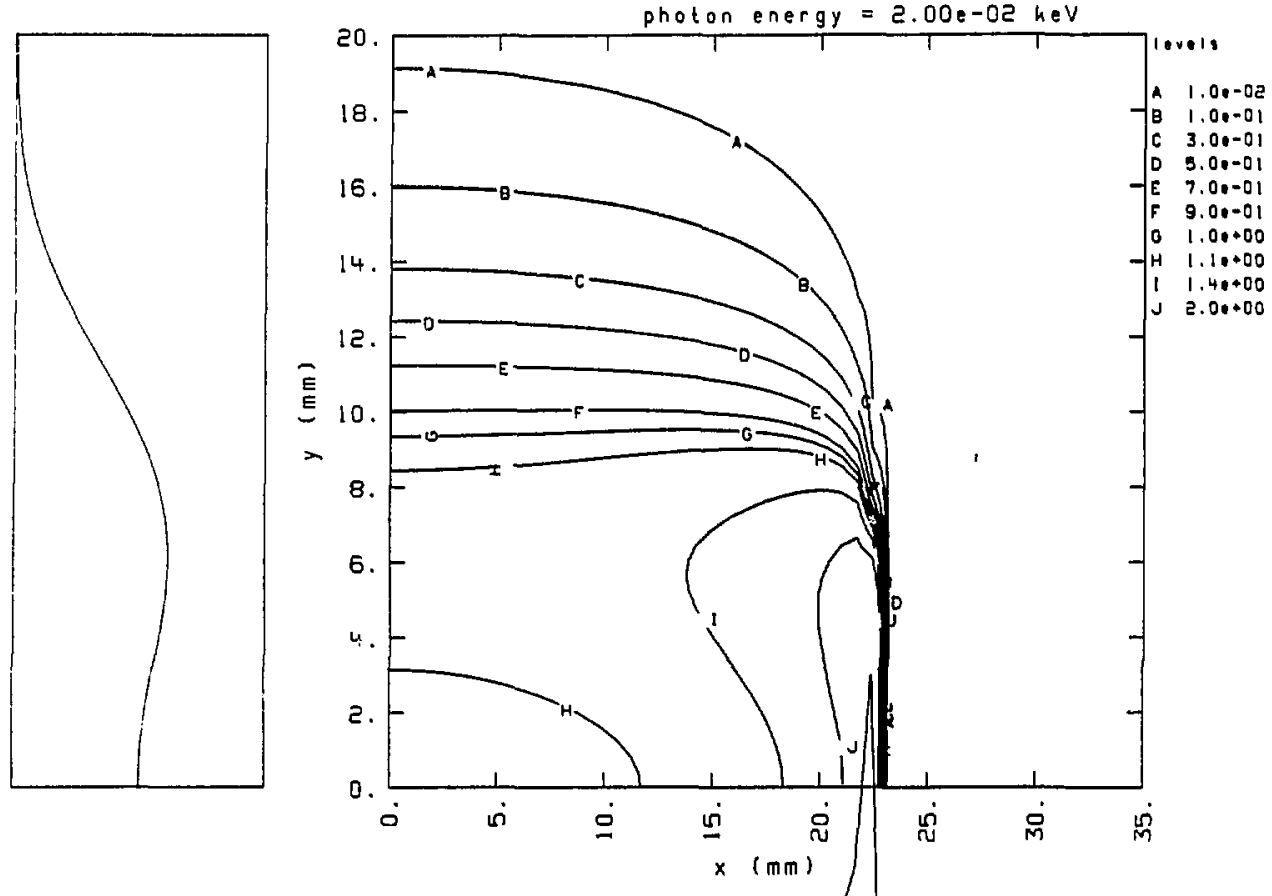

normalized to

$z=2.70 \mathrm{e}+09$

at $x=0$.

$y=0$.

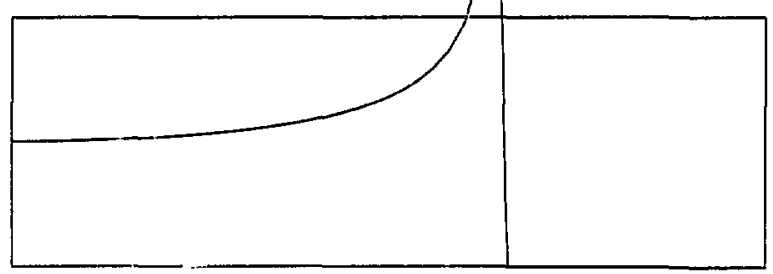

Fig. Al. 
wiggler: 30 poles; $B=13.0 \mathrm{hG} ; 1$ ambda $=12.85 \mathrm{~cm}$

8.5 m irom center of wiggler to. :rror

electron energy $=3.00 \mathrm{e}+00 \mathrm{GeV}$ critical energy $=7.78 \mathrm{G}+00 \mathrm{heV}$
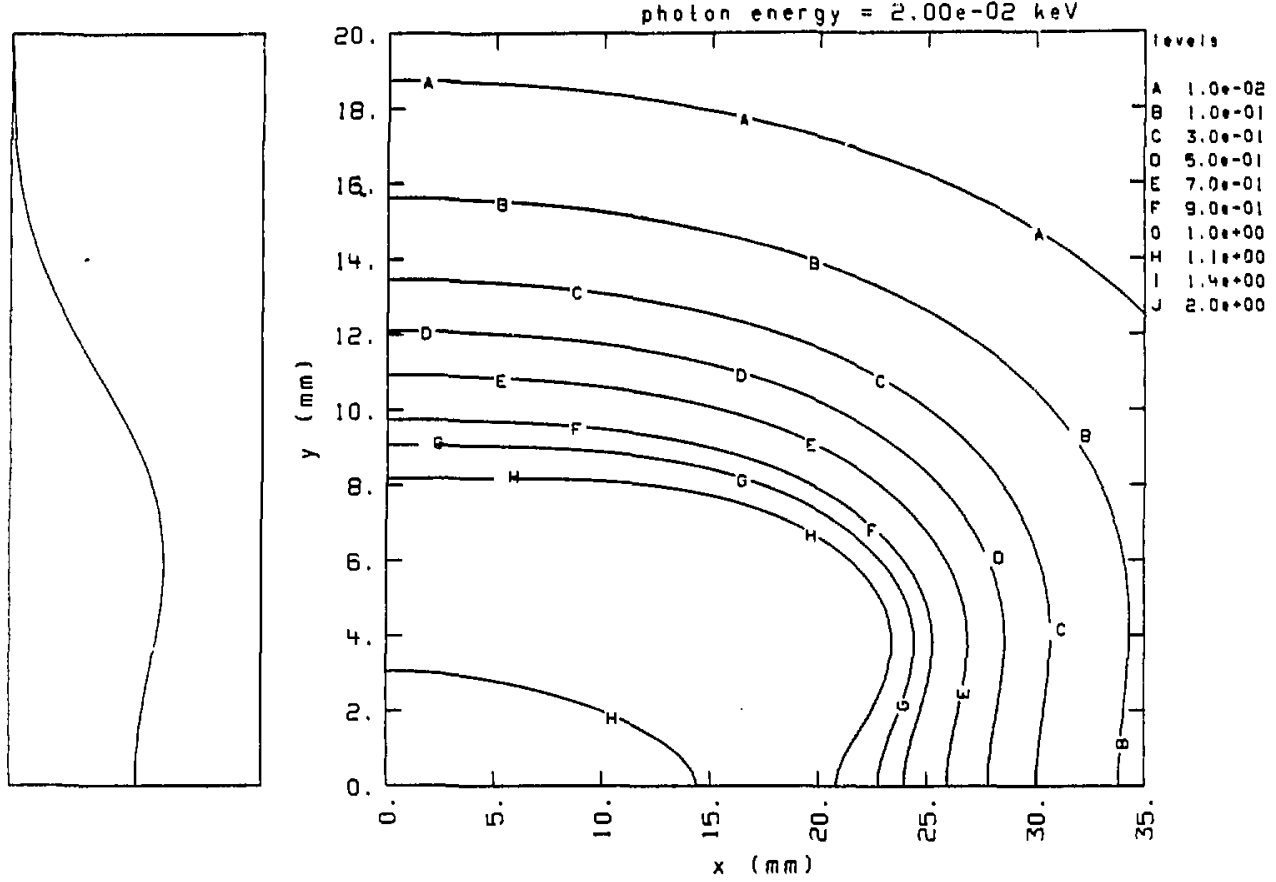

normalized to

$z=2.68 e+09$

at $x=0$.

$y=0$.

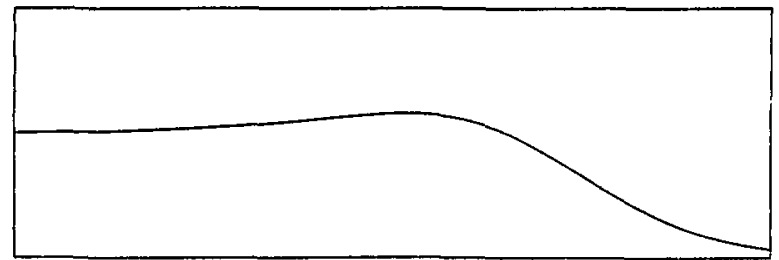

Fig. A2. 


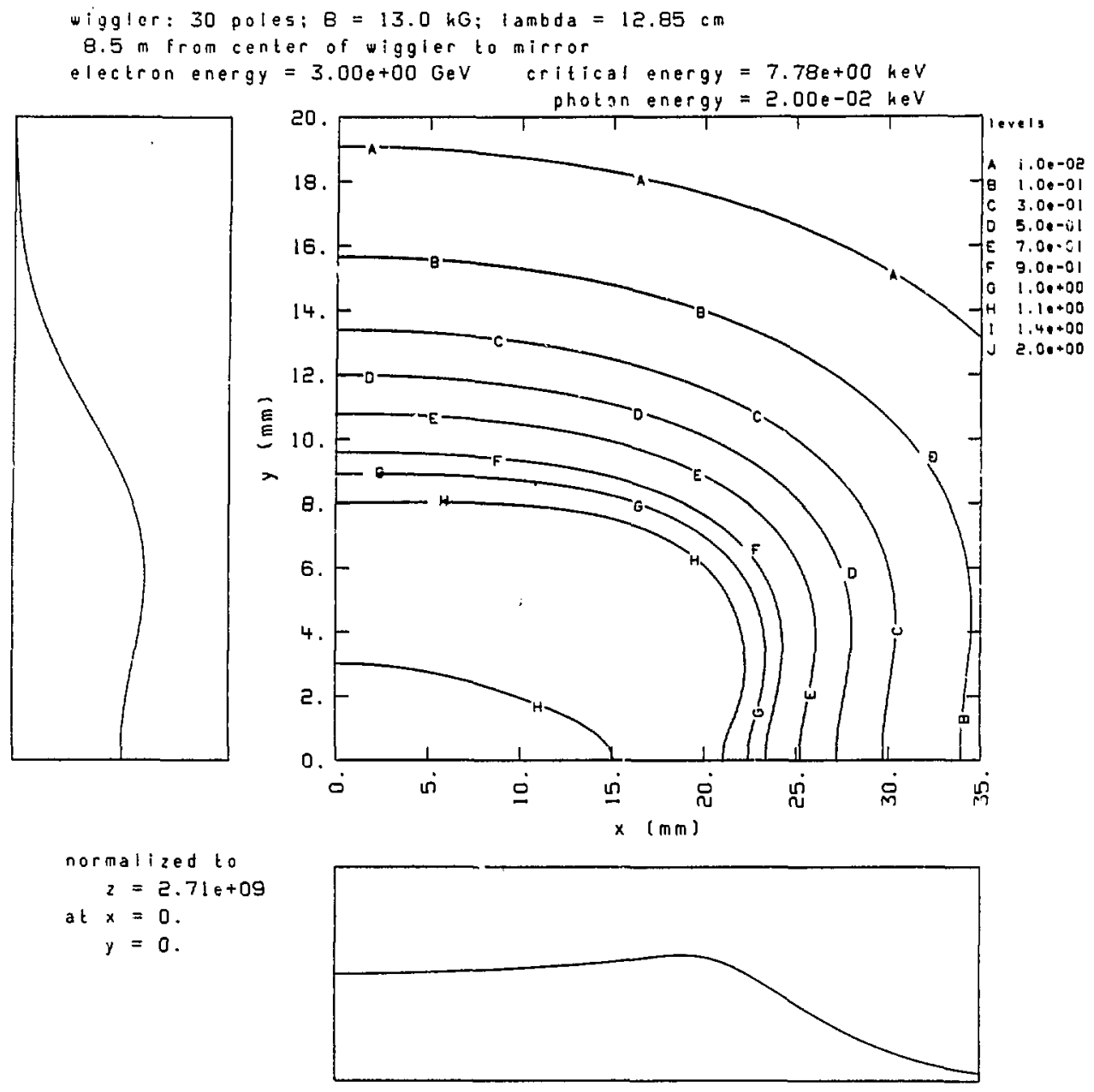

Fig. A3. 


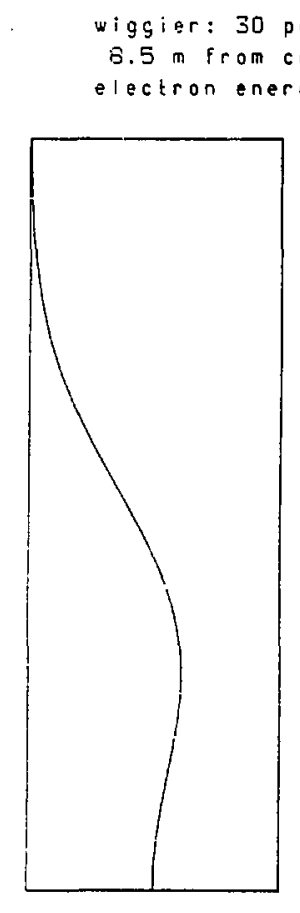

oles: $B=13.0 \mathrm{kG}$; lambda $=12.85 \mathrm{~cm}$

b.5 m from center of wiggler to mirror

electron energy $=3.00 e+00 \mathrm{GeV}$ critical energy $=7.78 \mathrm{e}+00 \mathrm{keV}$

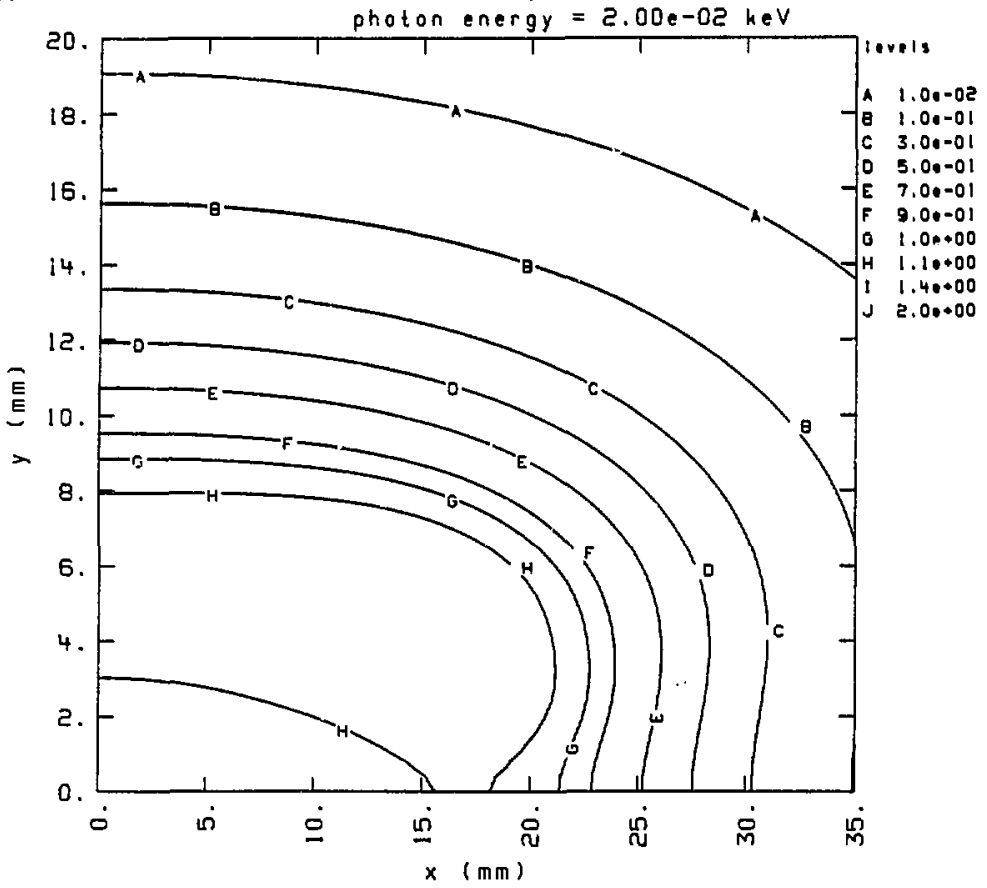

normalized to

$z=2.73 e+09$

at $x=0$.

$y=0$.

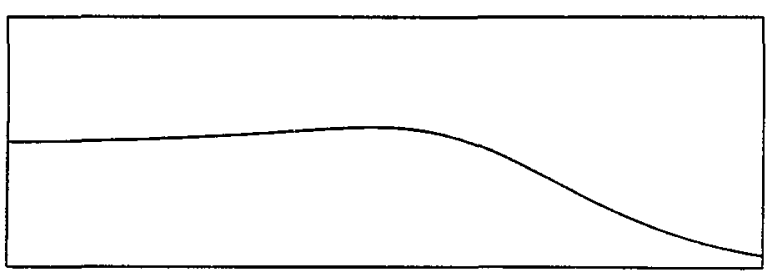

Fig. A4. 


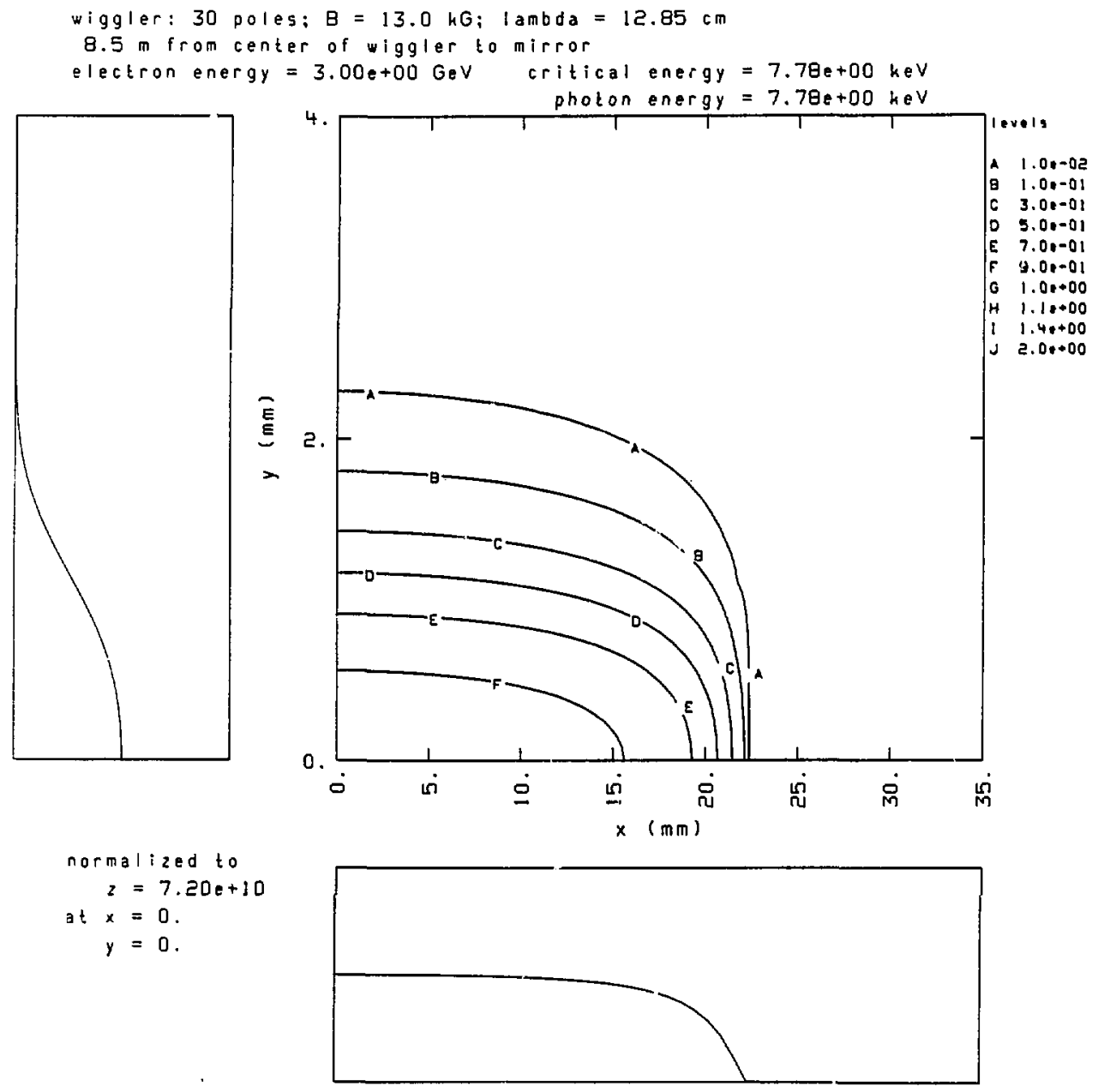

Fig. A5. 


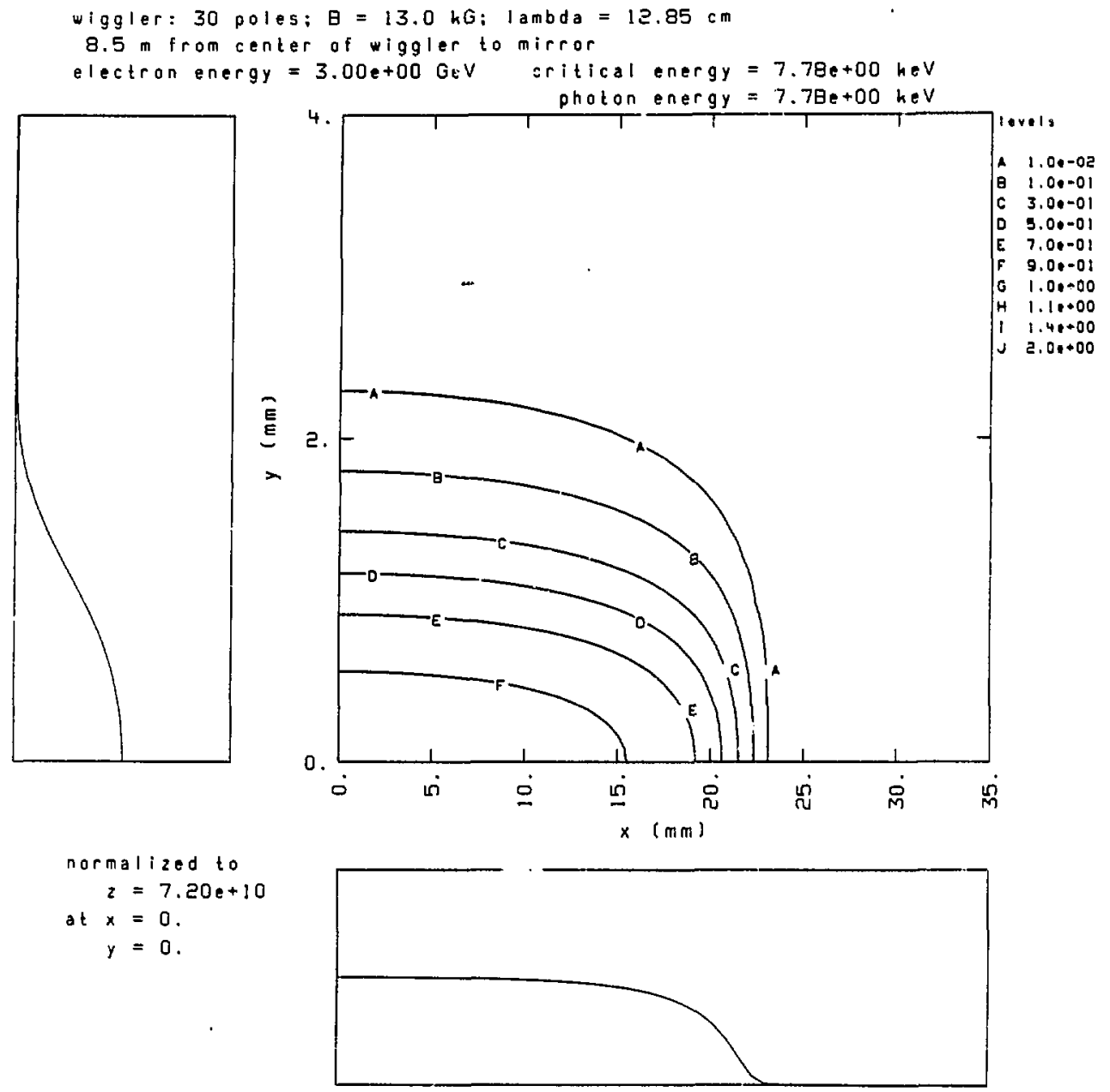

Fig. A6. 


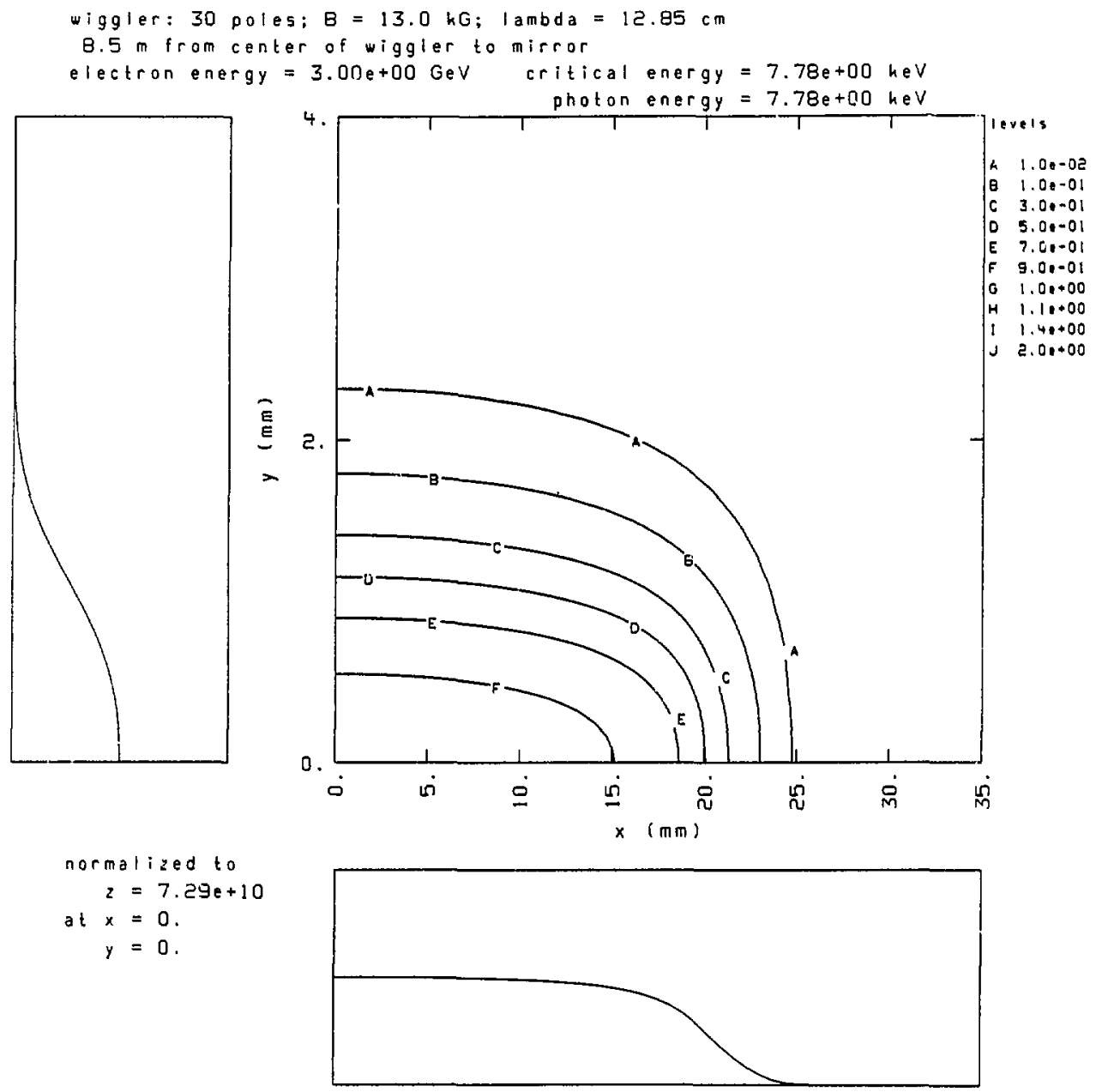

Fig. A7. 


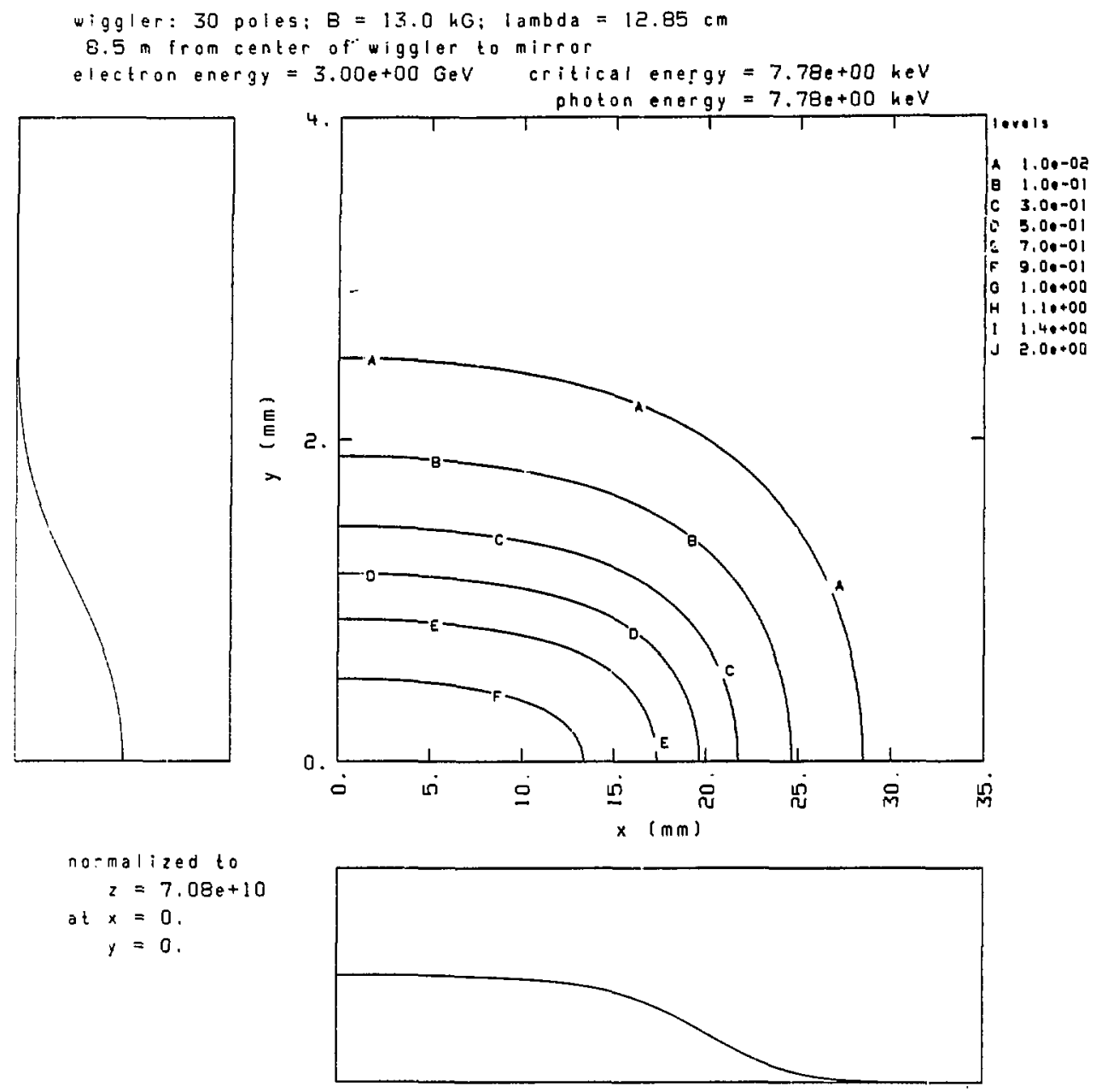

Fig. A8. 
List of figures:

Total intensity for $E=1.8 \mathrm{GeV}\left(\varepsilon_{c}=2.80 \mathrm{keV}\right)$

Figure

B1.1

B1.2

B1. 3

B1. 4

B1. 5

B1. 6

B1. 7
Photon Energy

$20 \mathrm{eV}$

$100 \mathrm{eV}$

$500 \mathrm{eV}$

$1 \mathrm{keV}$

$\varepsilon_{c} / 2$

$\varepsilon_{\mathrm{c}}$

$5 \varepsilon_{c}$
Page

18

19

20

21

22

23

24

Parallel polarization intensity for $E=1.8 \mathrm{GeV}\left(\varepsilon_{\mathrm{c}}=2.80 \mathrm{keV}\right)$

Figure

B2. 1

B2. 2

B2. 3

B2. 4

B2. 5

B2. 6

B2. 7
Photon Enery:

$20 \mathrm{eV}$

$500 \mathrm{eV}$

$1 \mathrm{keV}$

$\varepsilon_{\mathrm{c}} / 2$

$\varepsilon_{\mathrm{c}}$

$5 \varepsilon_{c}$

Page

25

26

27

28

29

30

31

Perpendicular polarization intensity for $E=1.8 \mathrm{GeV}\left(\varepsilon_{c}=2.80 \mathrm{keV}\right)$

Figure

B3.1

B3. 2

B 3.3

B3. 4

B3. 5

B3.6

B 3.7
Photon Energy

$20 \mathrm{eV}$

$100 \mathrm{eV}$

$5 \mathrm{CO}$ eV

$1 \mathrm{keV}$

$\varepsilon_{\mathrm{c}} / 2$

$\varepsilon_{c}$

$5 \varepsilon_{c}$
Page

33

34

35

36

37

38

Total intensity for $E=3.0 \mathrm{GeV}\left(\varepsilon_{c}=7.78 \mathrm{keV}\right)$

\begin{tabular}{lr} 
Figure & Photon Energy \\
\hline B 4.1 & $20 \mathrm{eV}$ \\
B 4.2 & $100 \mathrm{eV}$ \\
B4.3 & $500 \mathrm{eV}$ \\
B 4.4 & $1 \mathrm{keV}$ \\
B 4.5 & $\varepsilon_{\mathrm{c}} / 2$ \\
B 4.6 & $\varepsilon_{\mathrm{c}}$ \\
B4.7 & $5 \varepsilon_{\mathrm{c}}$
\end{tabular}


Parallel polarization intensity for $E=3.0 \mathrm{GeV}\left(\varepsilon_{\mathrm{c}}=7.78 \mathrm{keV}\right)$

\begin{tabular}{lrr} 
Figure & Photon Energy & Page \\
\cline { 2 - 2 } & $20 \mathrm{eV}$ & 46 \\
B5.2 & $100 \mathrm{eV}$ & 47 \\
B5.3 & $500 \mathrm{eV}$ & 48 \\
B5.4 & $1 \mathrm{keV}$ & 49 \\
B5.5 & $\varepsilon_{\mathrm{c}} / 2$ & 50 \\
B5.6 & $\varepsilon_{\mathrm{c}}$ & 51 \\
B5.7 & $5 \varepsilon_{\mathrm{c}}$ & 52
\end{tabular}

Perpendicular polarization intensity for $E=3.0 \mathrm{GeV}\left(\varepsilon_{\mathrm{c}}=7.78 \mathrm{keV}\right)$

\begin{tabular}{lrr} 
Figure & Photon Energy & Page \\
\cline { 2 - 2 } & $20 \mathrm{eV}$ & 53 \\
B6.2 & $100 \mathrm{eV}$ & 54 \\
B6.3 & $500 \mathrm{eV}$ & 55 \\
B6.4 & $1 \mathrm{keV}$ & 56 \\
B6.5 & $\varepsilon_{\mathrm{c}} / 2$ & 57 \\
B6.6 & $\varepsilon_{\mathrm{c}}$ & 58 \\
B6.7 & $5 \varepsilon_{\mathrm{c}}$ & 59
\end{tabular}

Total intensity for $E=3.4 \mathrm{GeV}\left(\varepsilon_{c}=10.00 \mathrm{keV}\right)$

\begin{tabular}{|c|c|}
\hline Figure & Photon Energy \\
\hline $\begin{array}{l}\text { B7.1 } \\
\text { B7.2 }\end{array}$ & $\begin{array}{r}20 \mathrm{eV} \\
100 \mathrm{eV}\end{array}$ \\
\hline $\begin{array}{l}\text { B7. } 3 \\
\text { B7. } 4\end{array}$ & $\begin{array}{r}500 \mathrm{eV} \\
1 \mathrm{keV}\end{array}$ \\
\hline $\begin{array}{l}\text { B7. } 5 \\
\text { B7.6 } \\
\text { B7.7 }\end{array}$ & $\begin{array}{r}\varepsilon_{c} / 2 \\
\varepsilon_{c} \\
5 \varepsilon_{c}\end{array}$ \\
\hline
\end{tabular}

Parallel polarization intensity for $E=3.4 \mathrm{GeV}\left(\varepsilon_{\mathrm{c}}=10.00 \mathrm{keV}\right)$

\begin{tabular}{lr} 
Figure & Photon Energy \\
\hline B8.1 & $20 \mathrm{eV}$ \\
B8.2 & $100 \mathrm{eV}$ \\
B8.3 & $500 \mathrm{eV}$ \\
B8.4 & $1 \mathrm{keV}$ \\
B8.5 & $\varepsilon_{\mathrm{c}} / 2$ \\
B8.6 & $\varepsilon_{\mathrm{c}}$ \\
B8.7 & $5 \varepsilon_{\mathrm{c}}$
\end{tabular}

Perpendicular polarization intensity for $E=3.4 \mathrm{GeV}\left(\varepsilon_{\mathrm{c}}=10.00 \mathrm{keV}\right)$

\begin{tabular}{lr} 
Figure & Photon Energy \\
\cline { 2 - 2 } B9.1 & $20 \mathrm{eV}$ \\
B9.2 & $100 \mathrm{eV}$ \\
B9.3 & $500 \mathrm{eV}$ \\
B9.4 & $1 \mathrm{keV}$ \\
B9.5 & $\varepsilon_{\mathrm{c}} / 2$ \\
B9.6 & $\varepsilon_{\mathrm{c}}$ \\
B9.7 & $5 \varepsilon_{\mathrm{C}}$
\end{tabular}




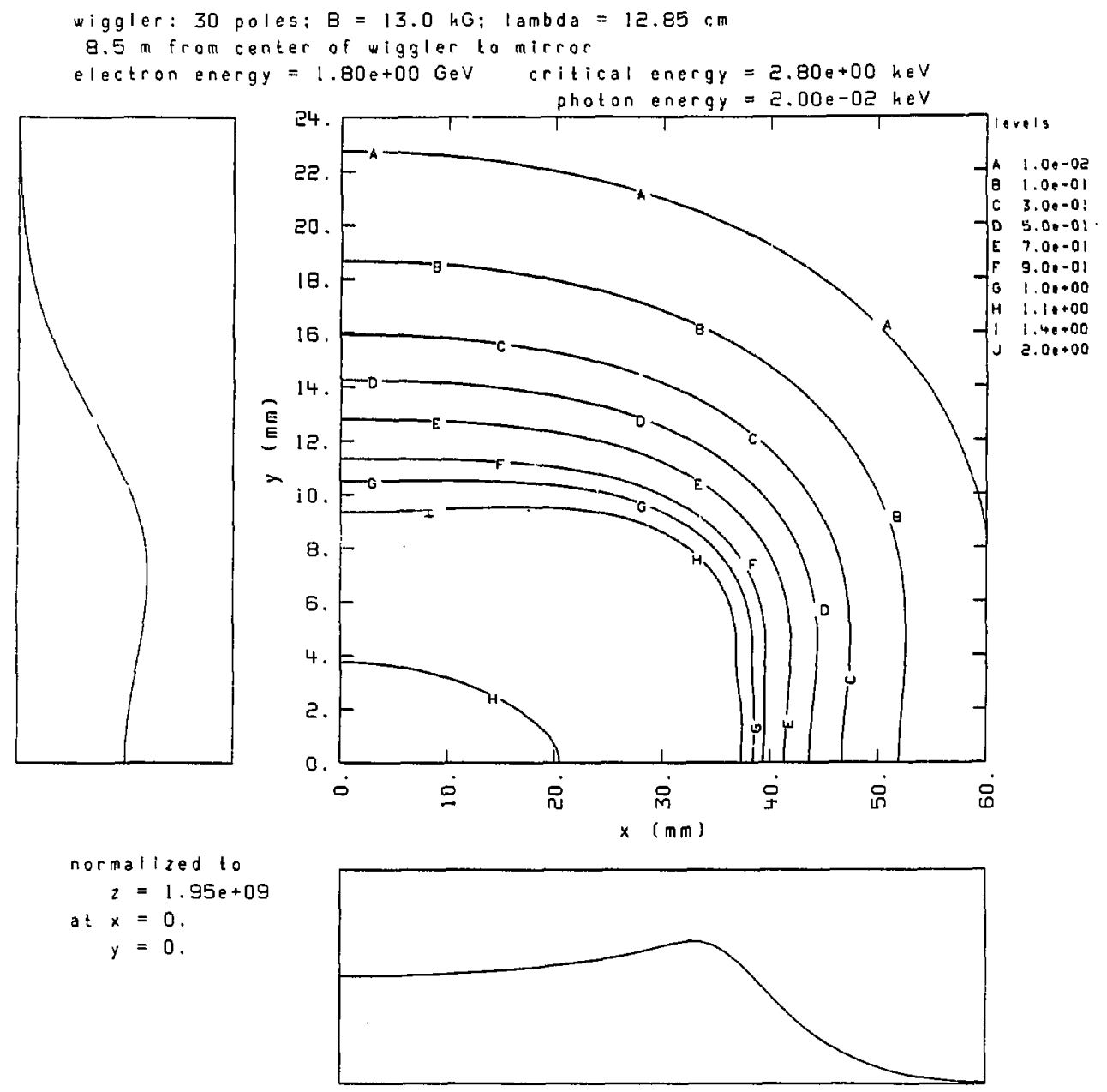

Fig. B1.1. 


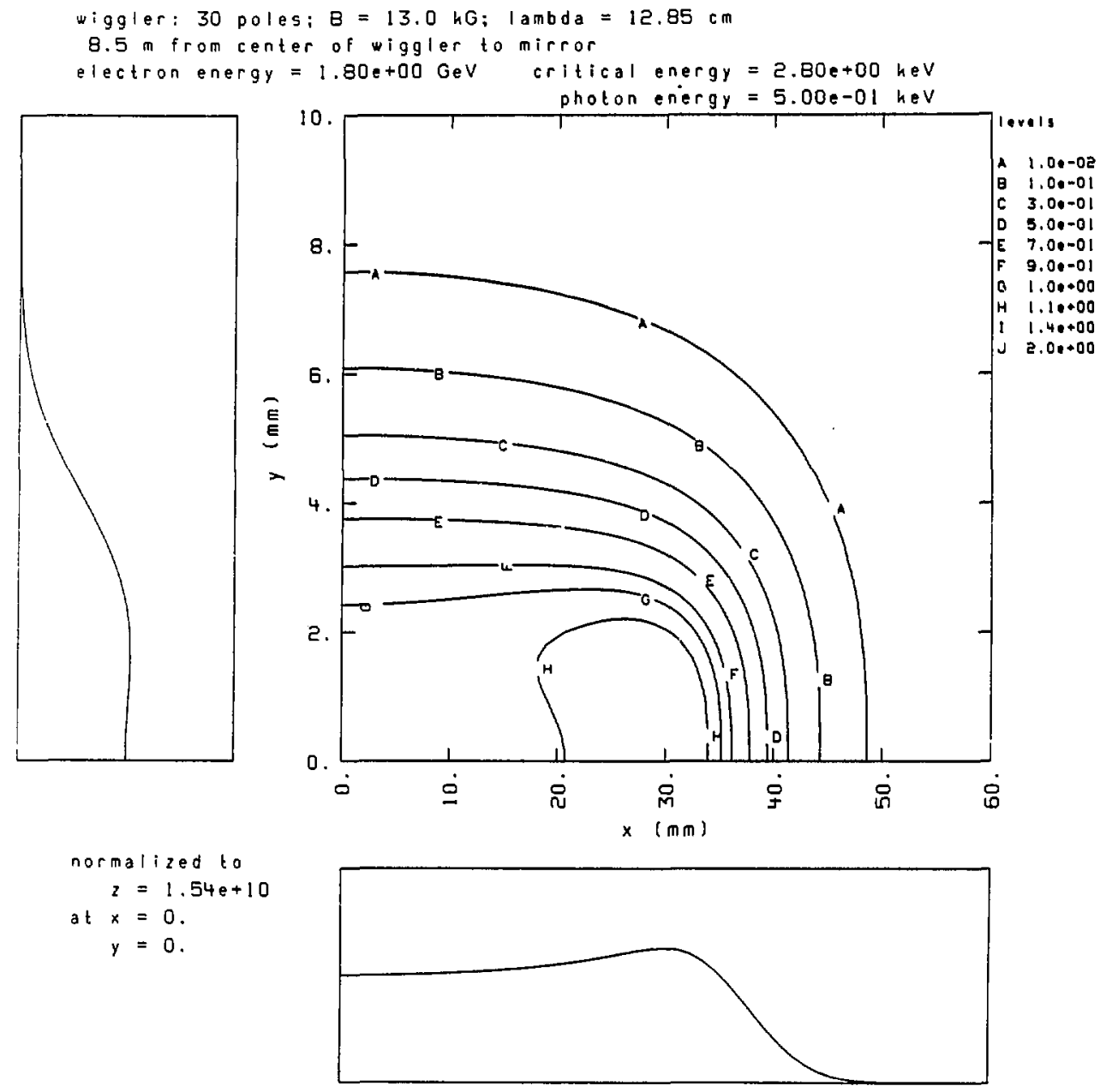

Fig. Bl.2. 


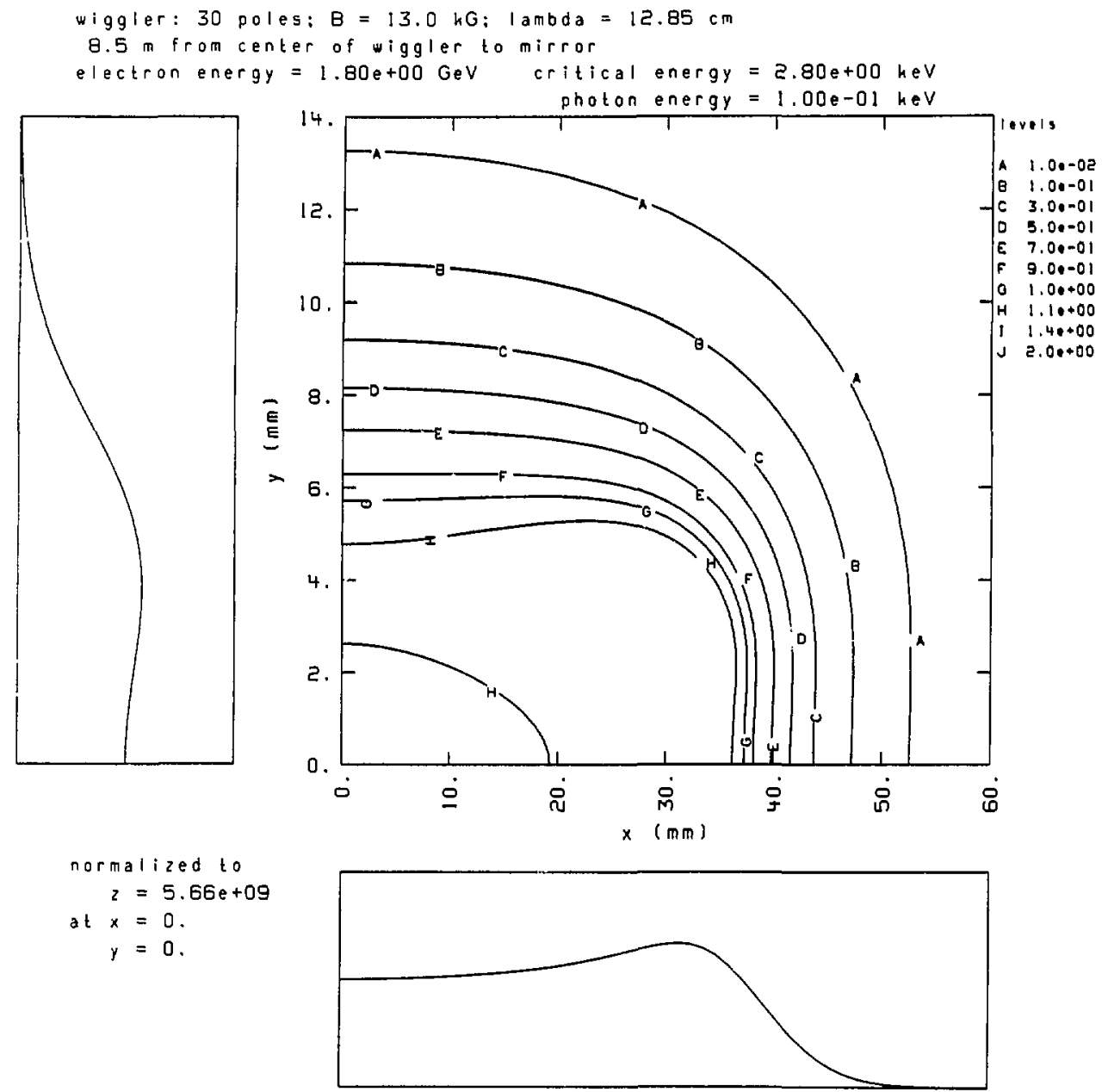

Fig. B1.3. 


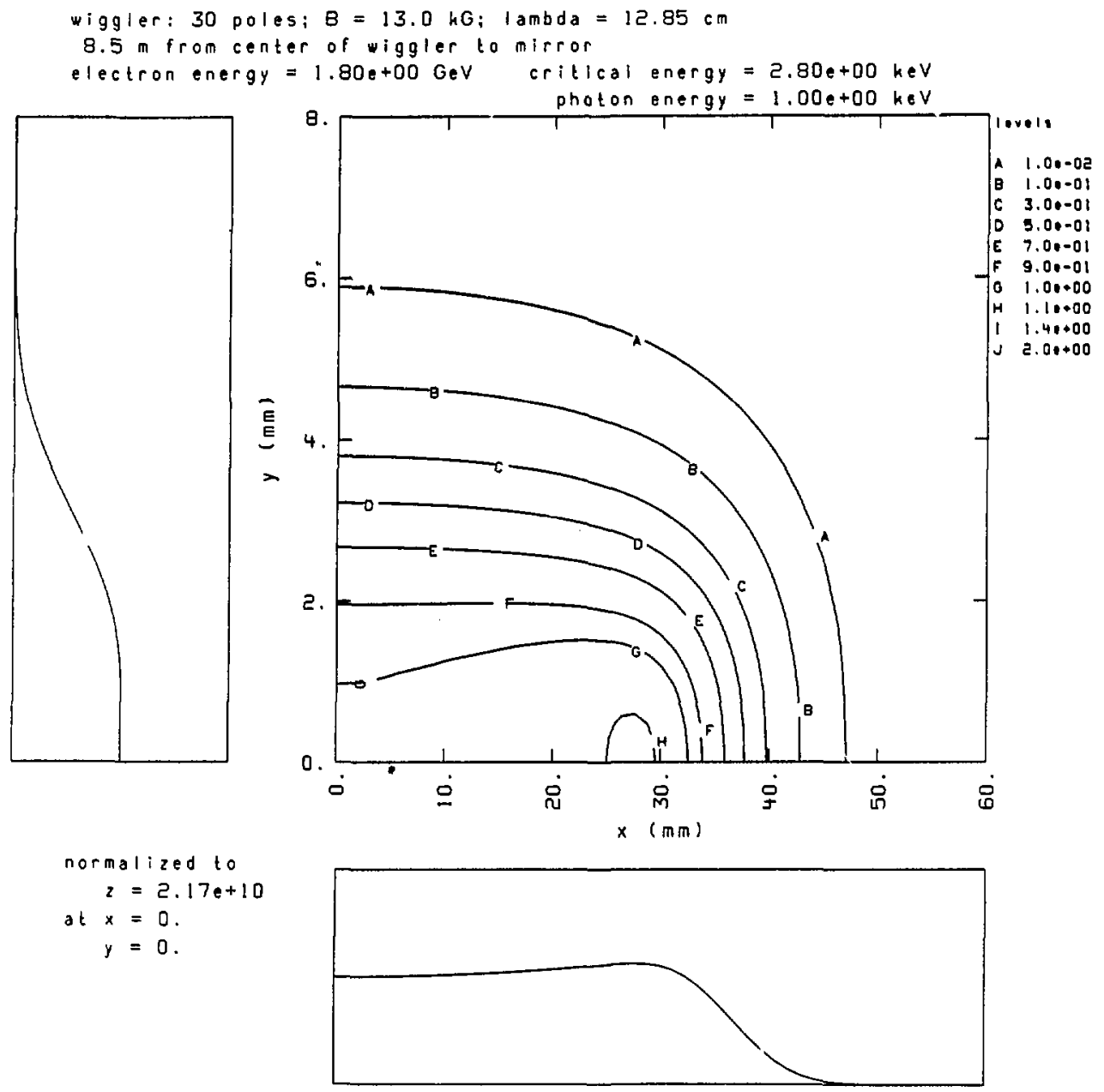

Fig. B1.4. 


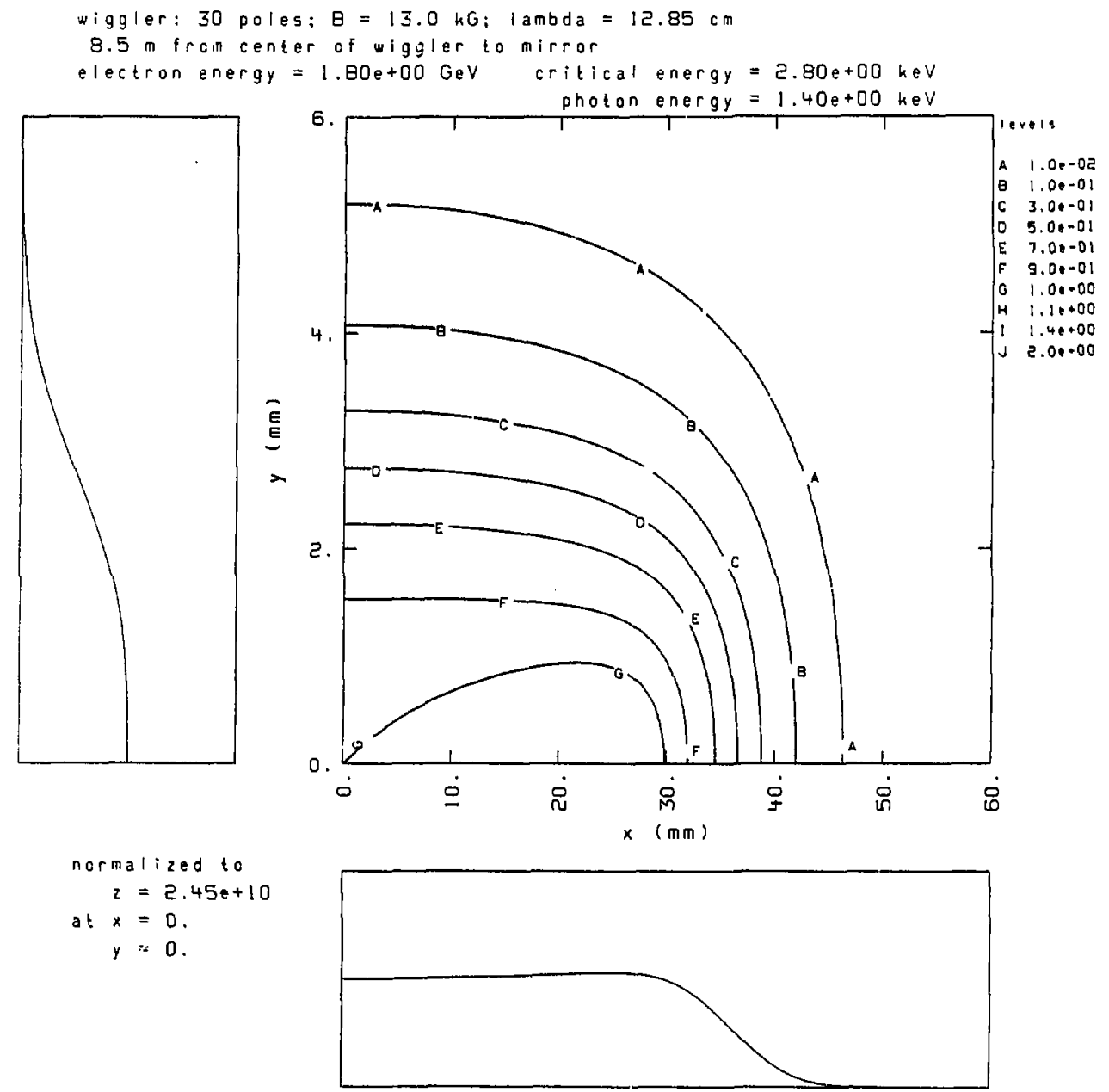

Fig. 81.5 . 


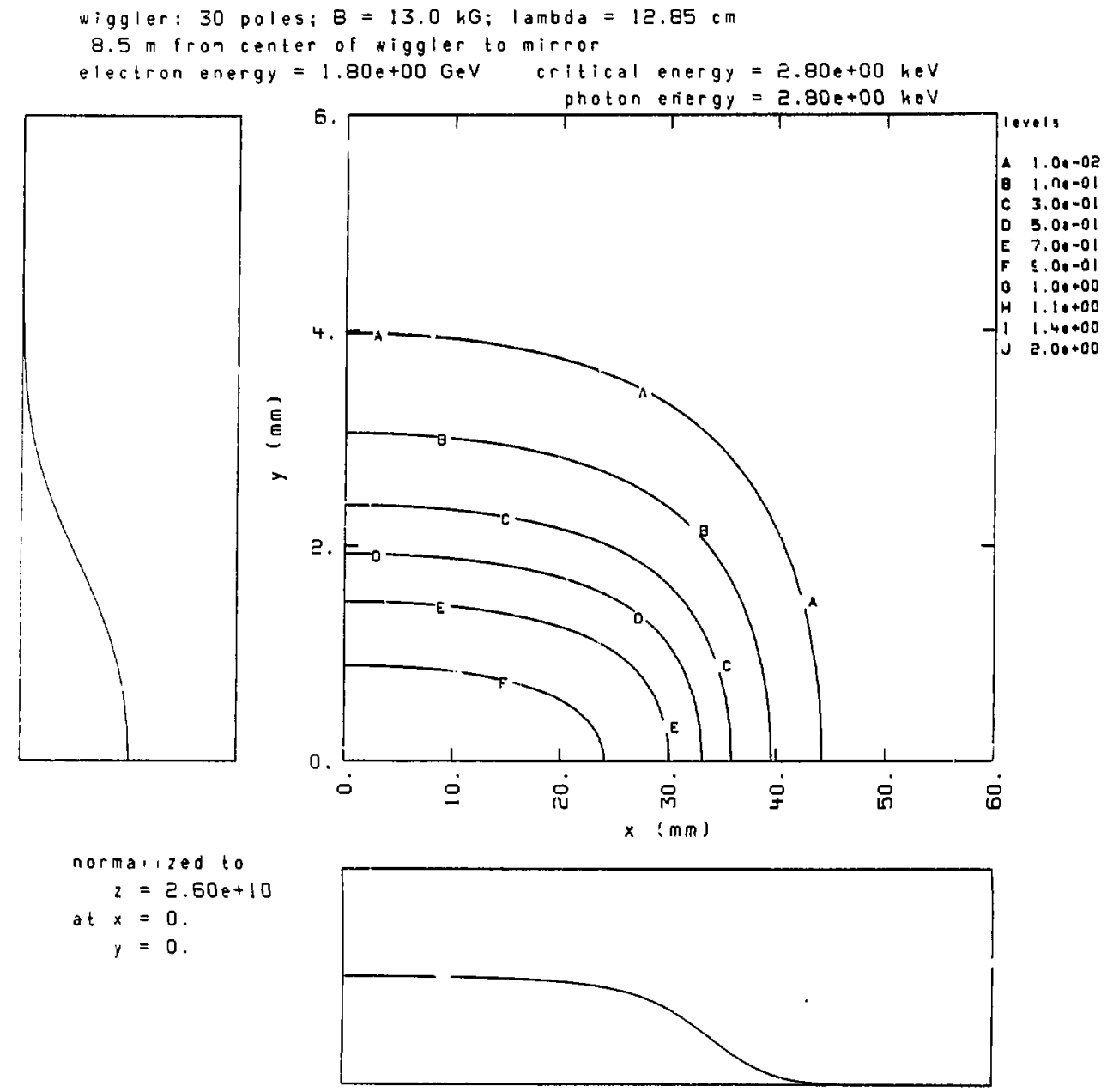

Fig. B1.6. 


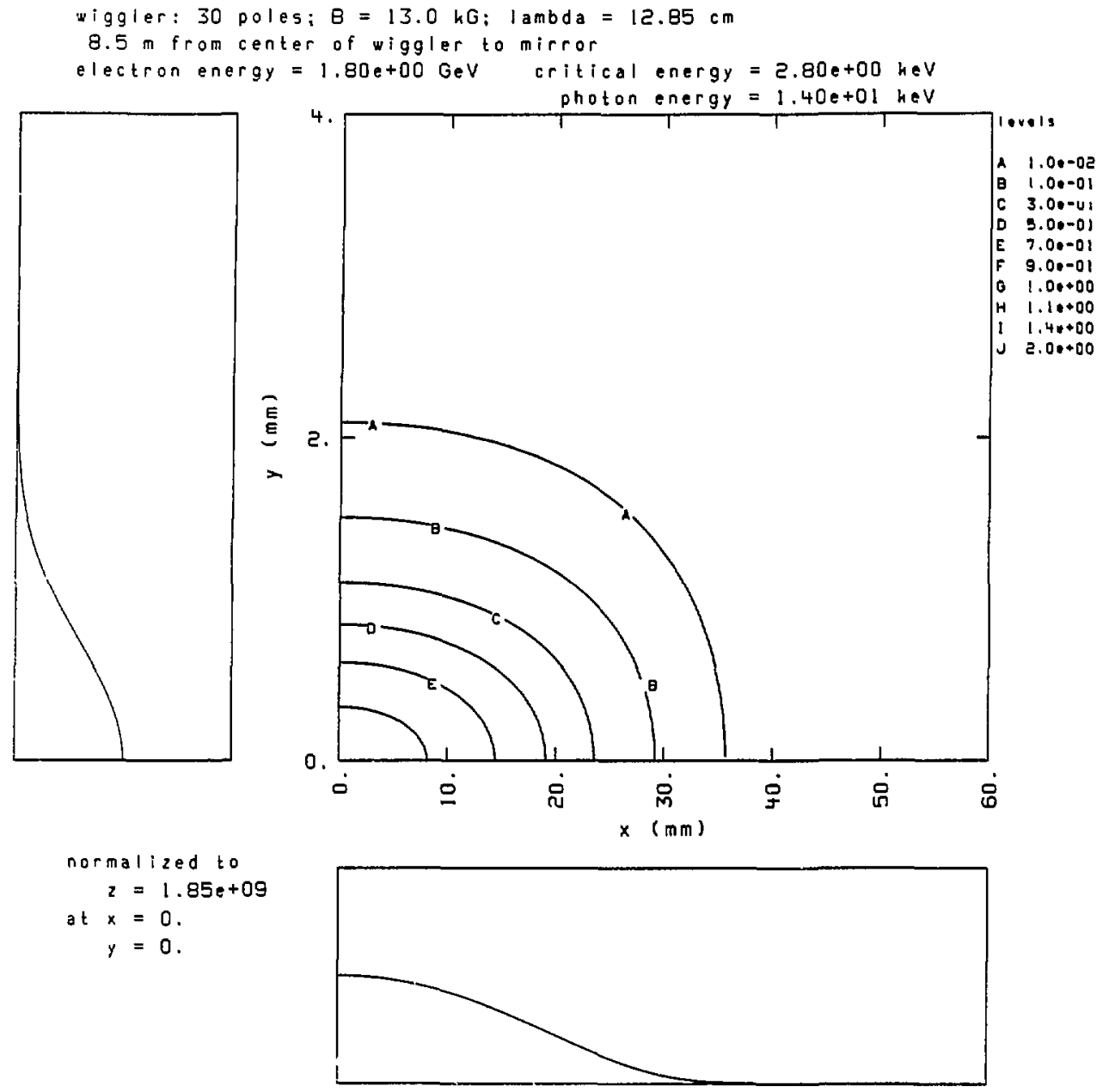

Fig. B1.7. 


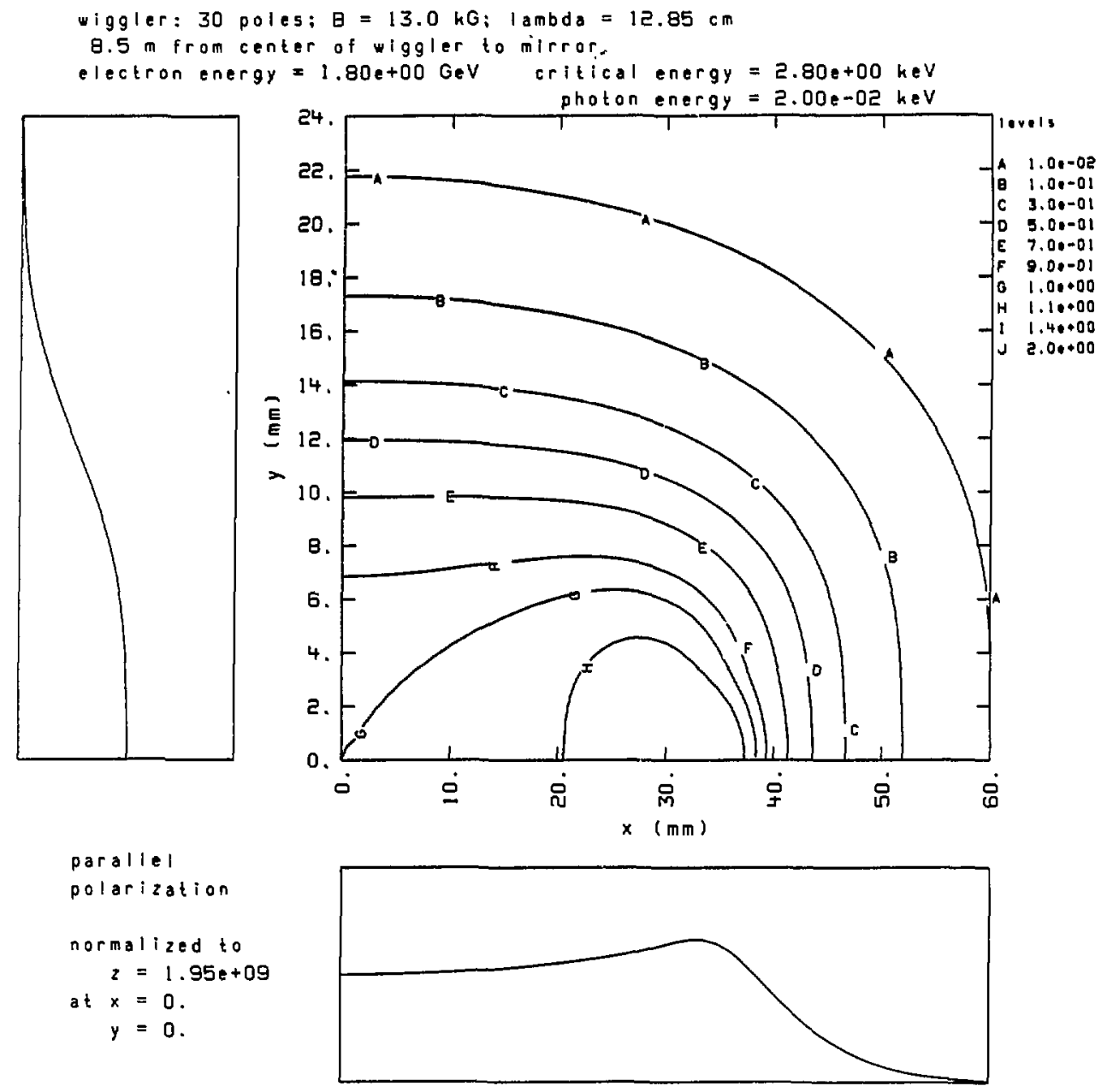

Fig. B2.1. 
wiggler: 30 poles: $B=13.0 \mathrm{hG} ; \mathrm{lambda}=12.85 \mathrm{~cm}$

$8.5 \mathrm{~m}$ from center of wiggler to mirror

electron energy $=1.80 \mathrm{e}+00 \mathrm{GeV}$ critical energy $=2.80 \mathrm{e}+00 \mathrm{heV}$
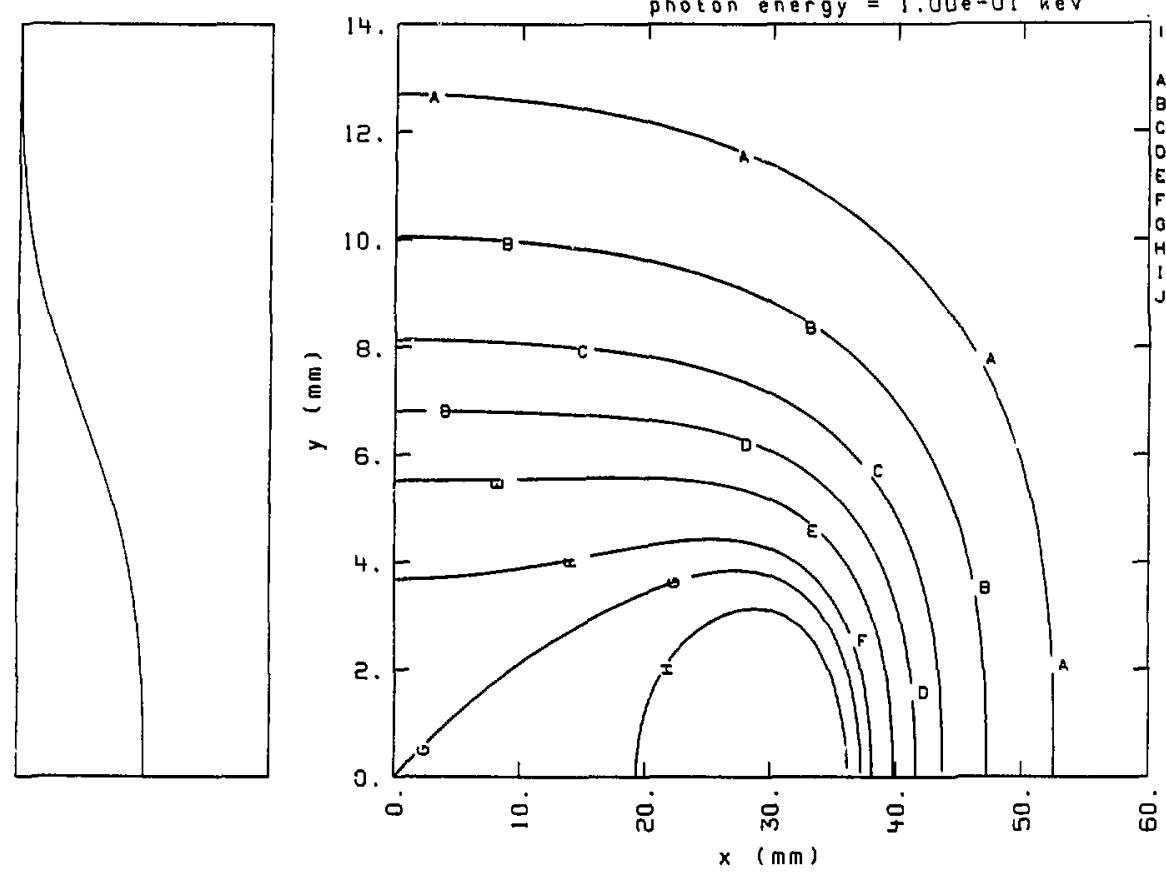

paral Iel

polarization

normalized to

$z=5.65 *+09$

at $x=0$.

$y=0$.

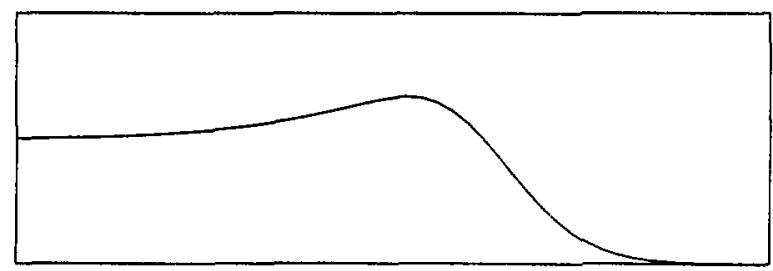

Fig. B2.2. 


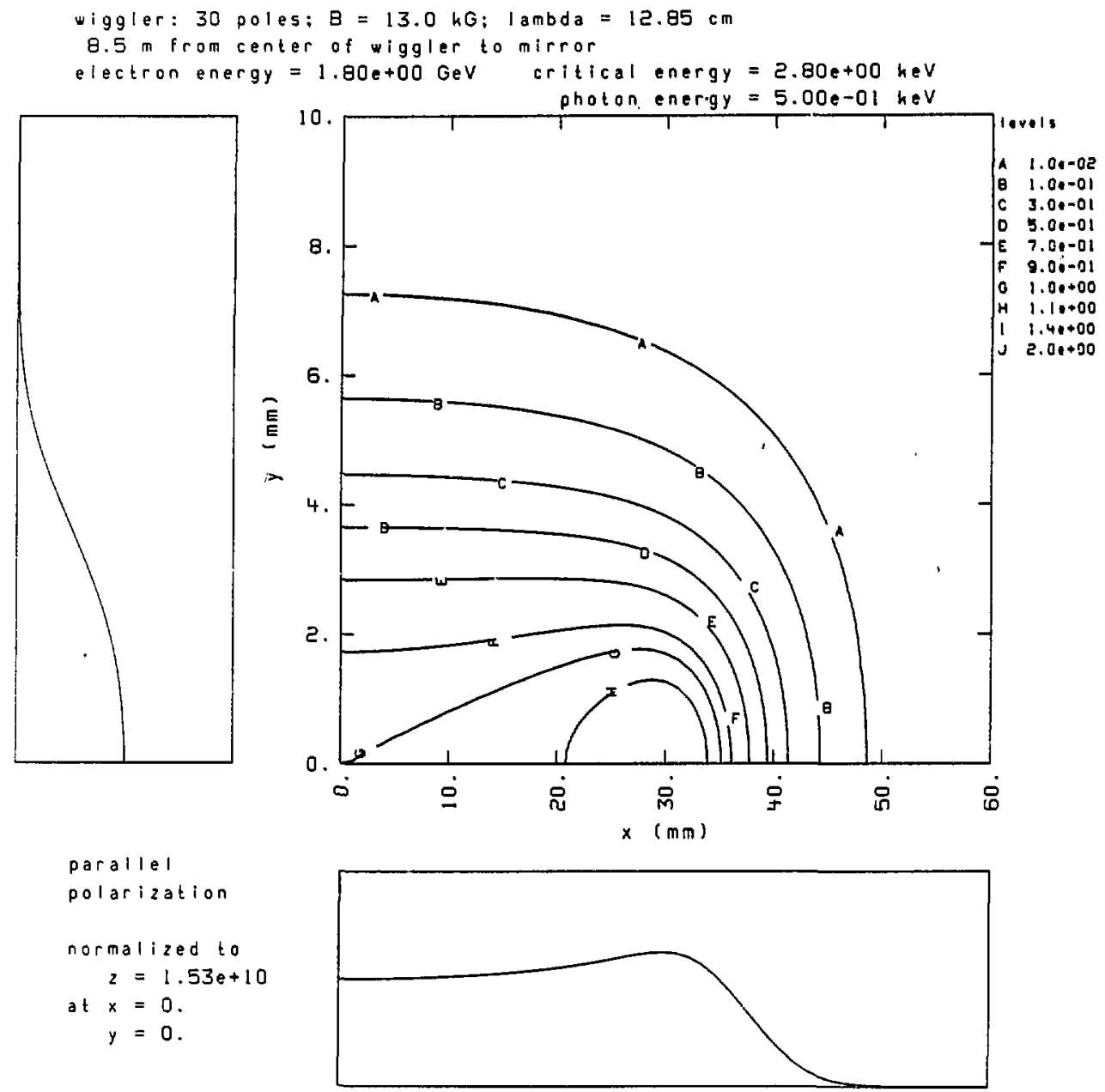

Fig. B2.3. 

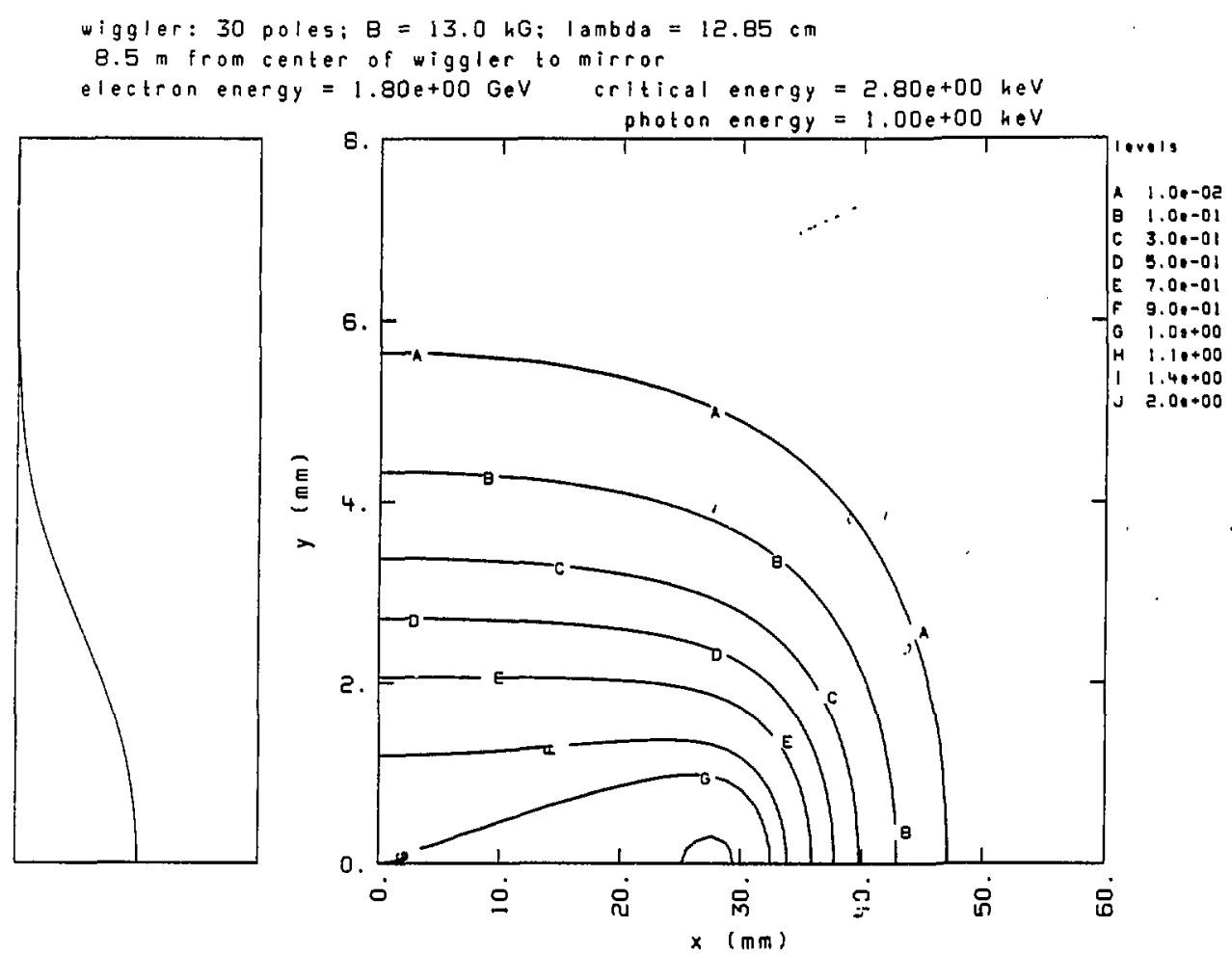

parallel

polarization

normalized to

$z=2.16 e+10$

at $x=0$.

$y=0$.

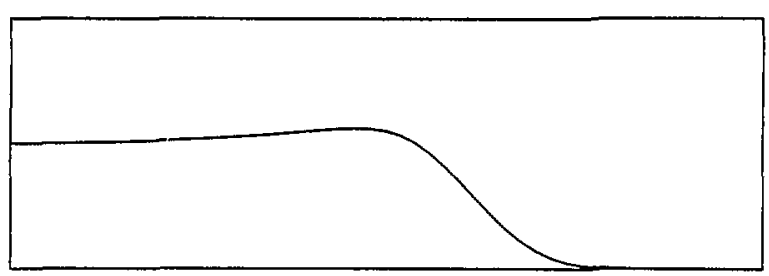

Fig. B2.4. 


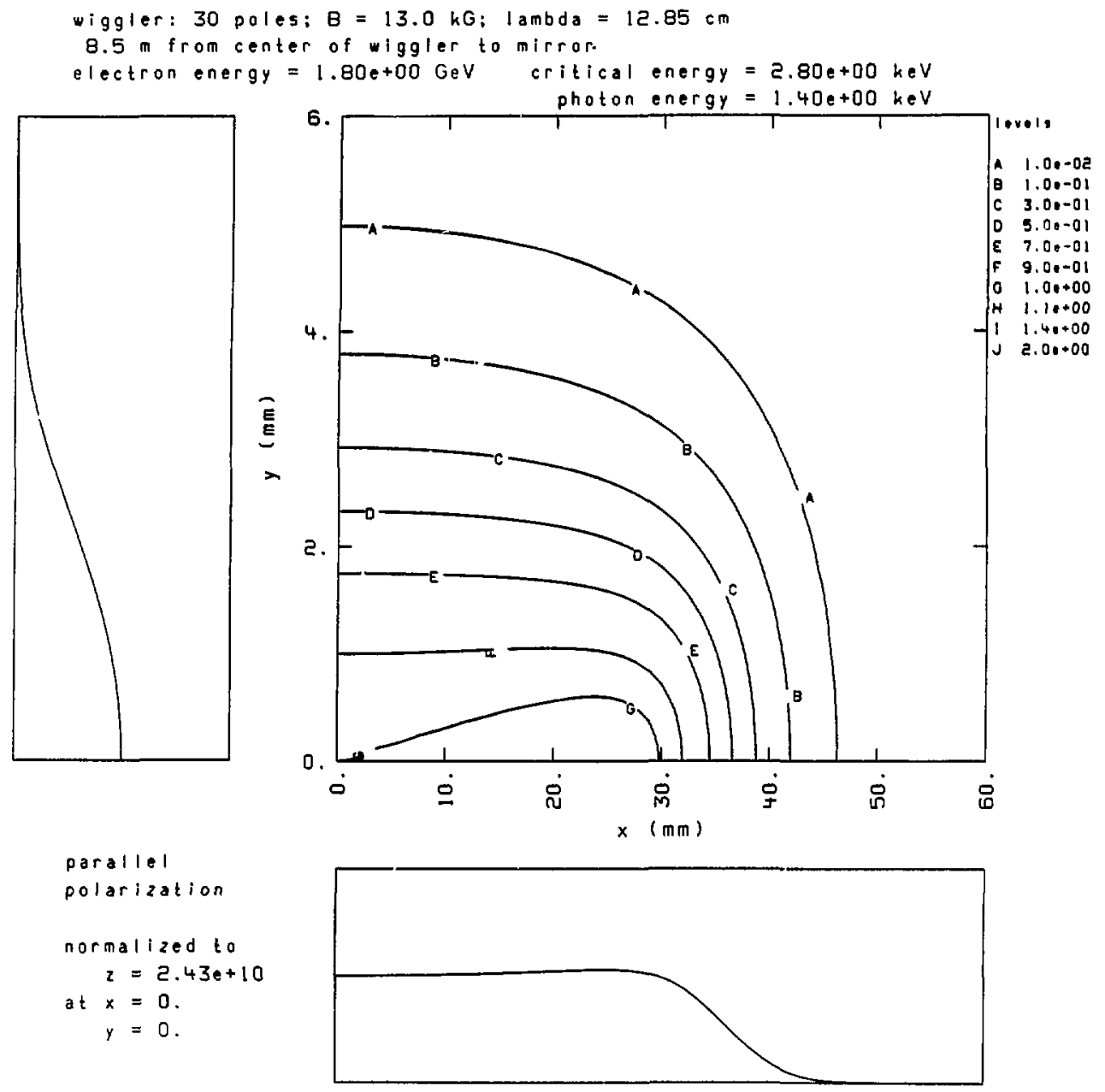

Fig. B2.5. 


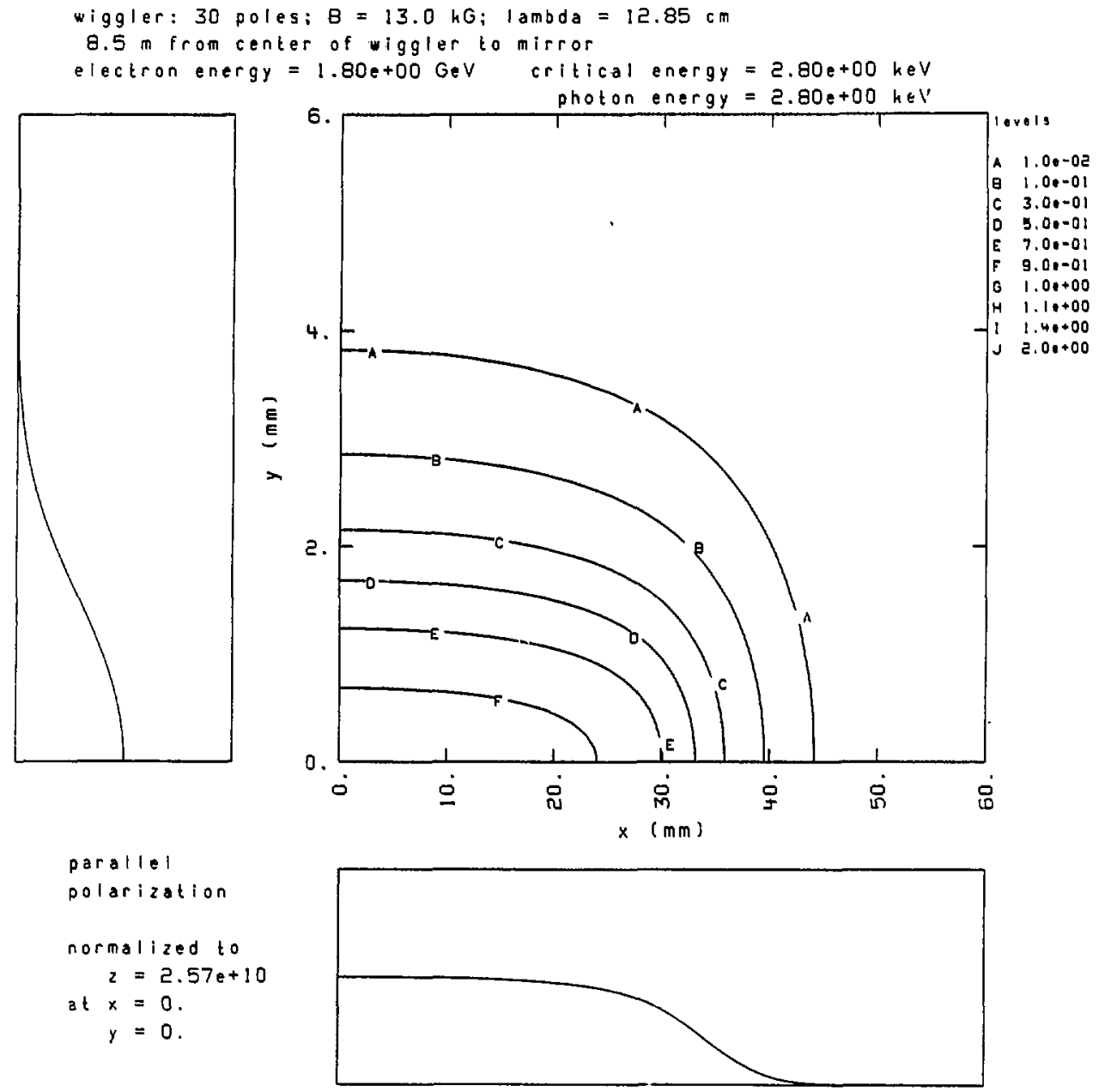

Fig. B2.6. 


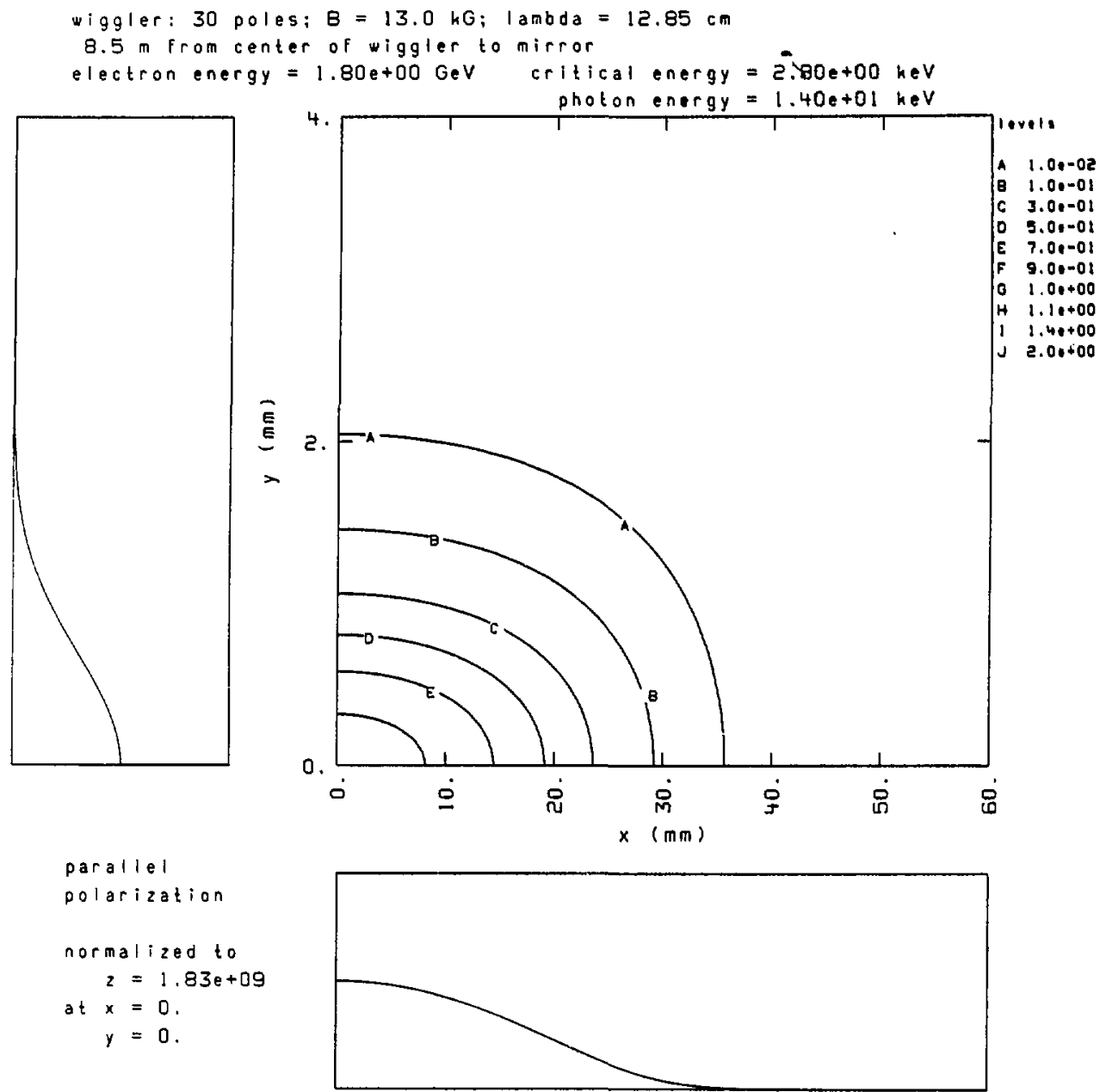

Fig. B2.7. 


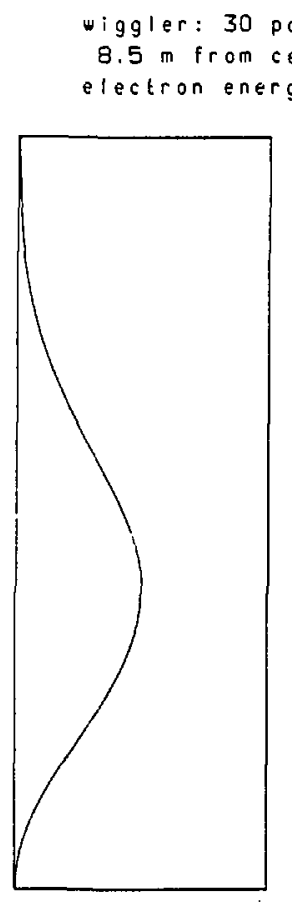

$$
\begin{aligned}
& \text { perpendicular } \\
& \text { polarization } \\
& \text { normalized to } \\
& z=7.25 e+08 \\
& \begin{aligned}
\text { at } x & =0 . \\
y & =9.60 e+00
\end{aligned}
\end{aligned}
$$$$
\text { oles: } B=13.0 \mathrm{kG} ; \quad \text { ambda }=12.85 \mathrm{~cm}
$$$$
\begin{aligned}
9 y=1.80 \mathrm{e}+00 \mathrm{GeV} \quad \mathrm{critical} \text { energy } & =2.80 \mathrm{e}+00 \mathrm{keV} \\
\text { photon energy } & =2.00 \mathrm{e}-02 \mathrm{keV}
\end{aligned}
$$
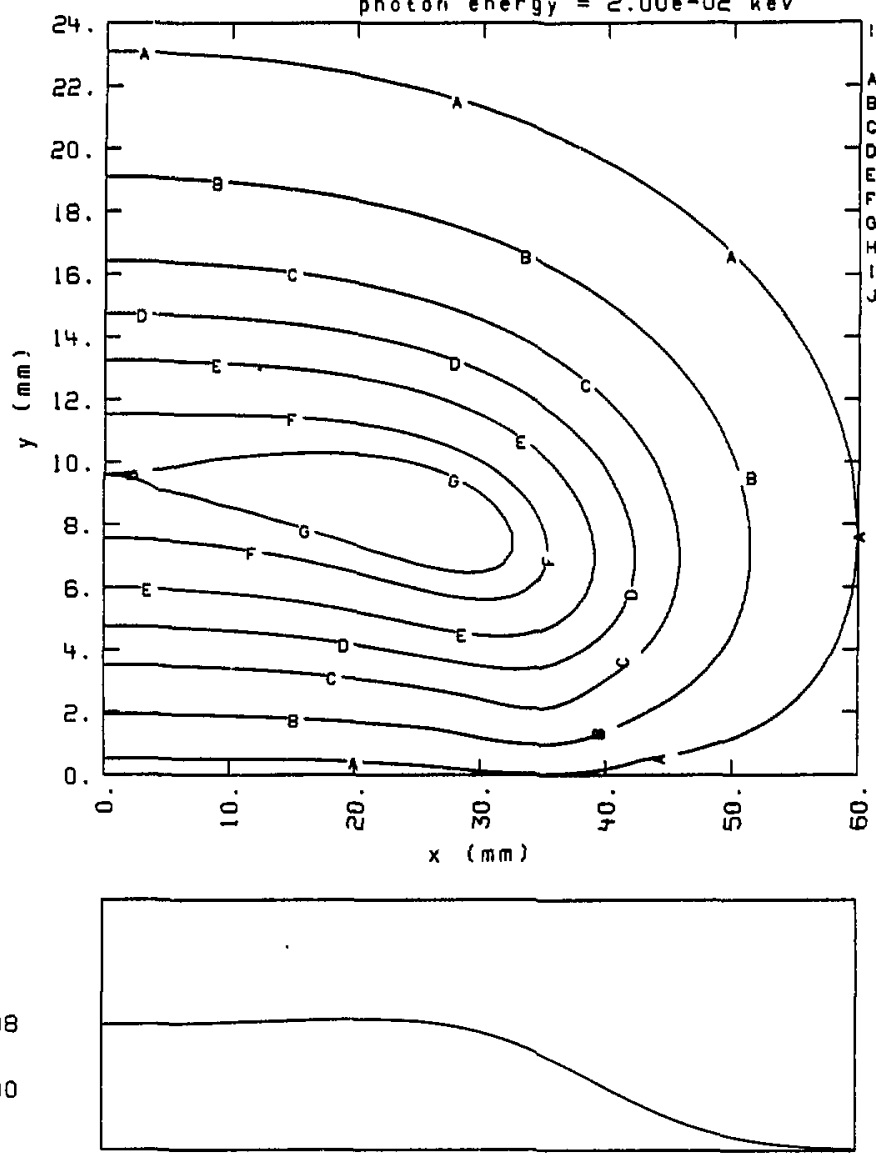

Fig. B3.1. 


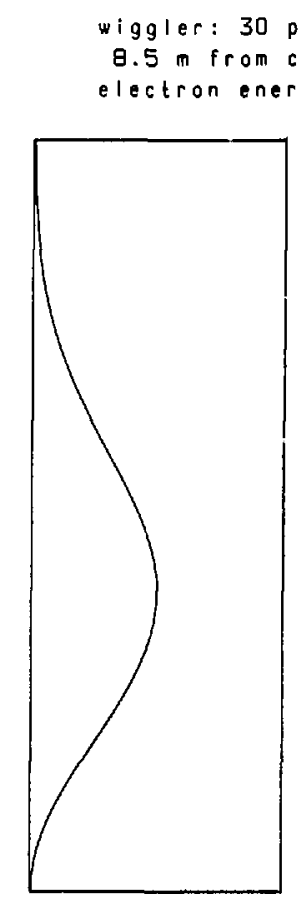

perpendicular nolarization

normalized to

$z=1.88 e+09$

at $x=0$.

$y=5.60 e+00$

01 es; $B=13.0 \mathrm{kG} ; 1 \mathrm{ambda}=12.85 \mathrm{~cm}$

$g y=1.800+00 \mathrm{GeV}$ critical energy $=2.80 \mathrm{e}+00 \mathrm{heV}$

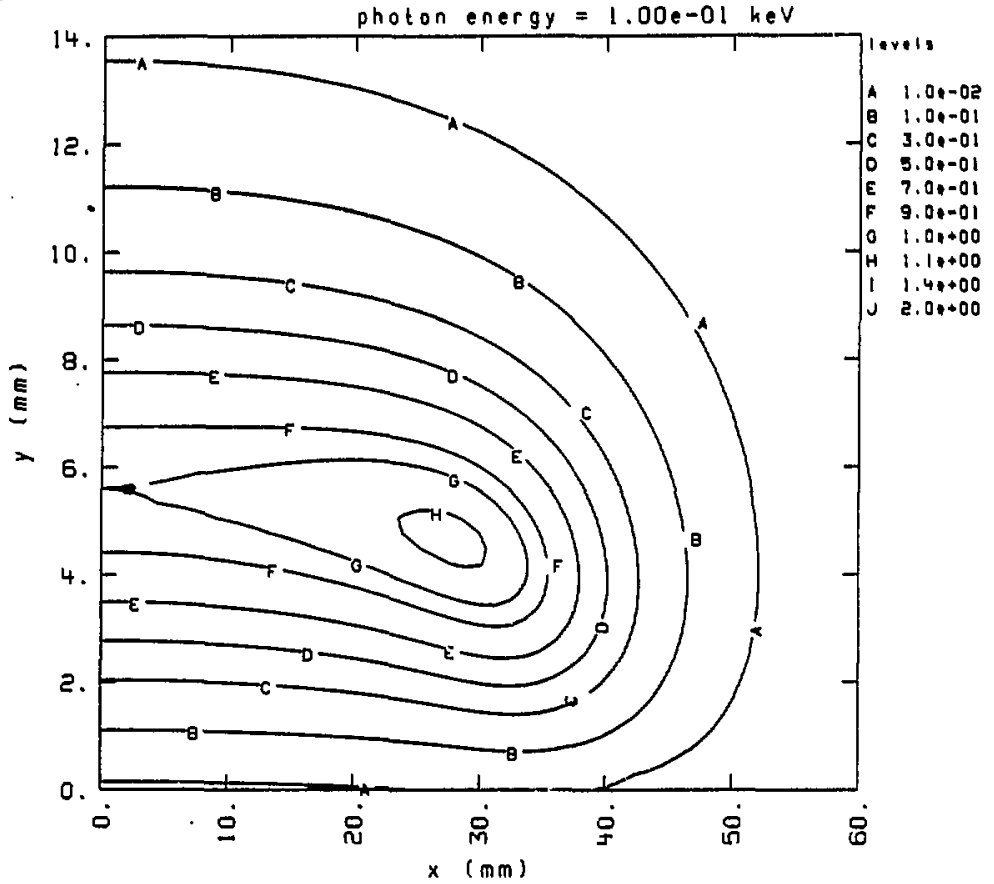

$x(\mathrm{~mm})$

Fig. B3.2. 


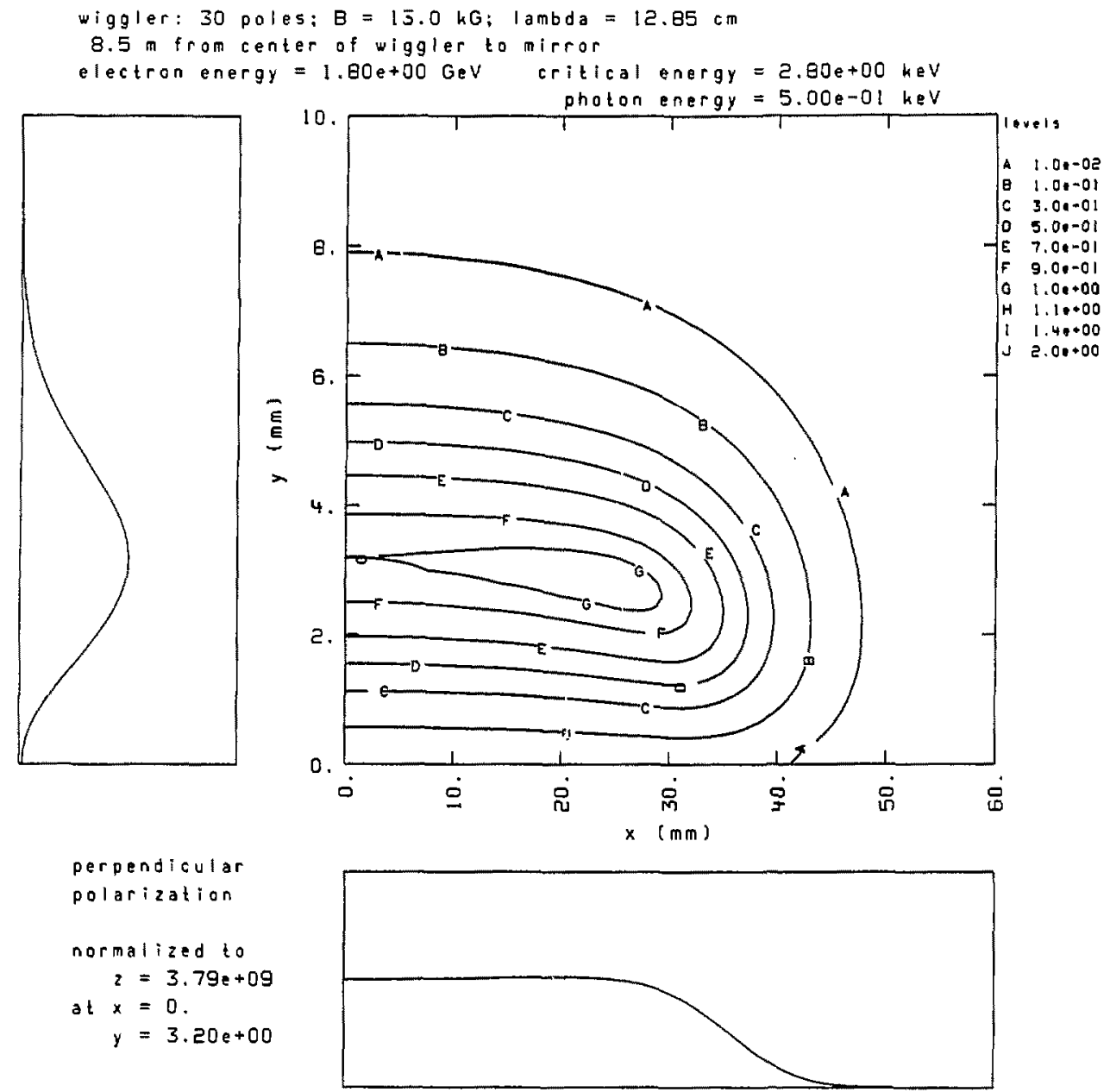

Fig. B3.3. 
wiggler: 30 poles: $B=13.0 \mathrm{kG}: 1 \mathrm{ambda}=12.85 \mathrm{~cm}$

$9.5 \mathrm{~m}$ from center of wiggler to mirror

electron energy $=1.80$ e+00 GeV critical energy $=2.80$ e 00 heV
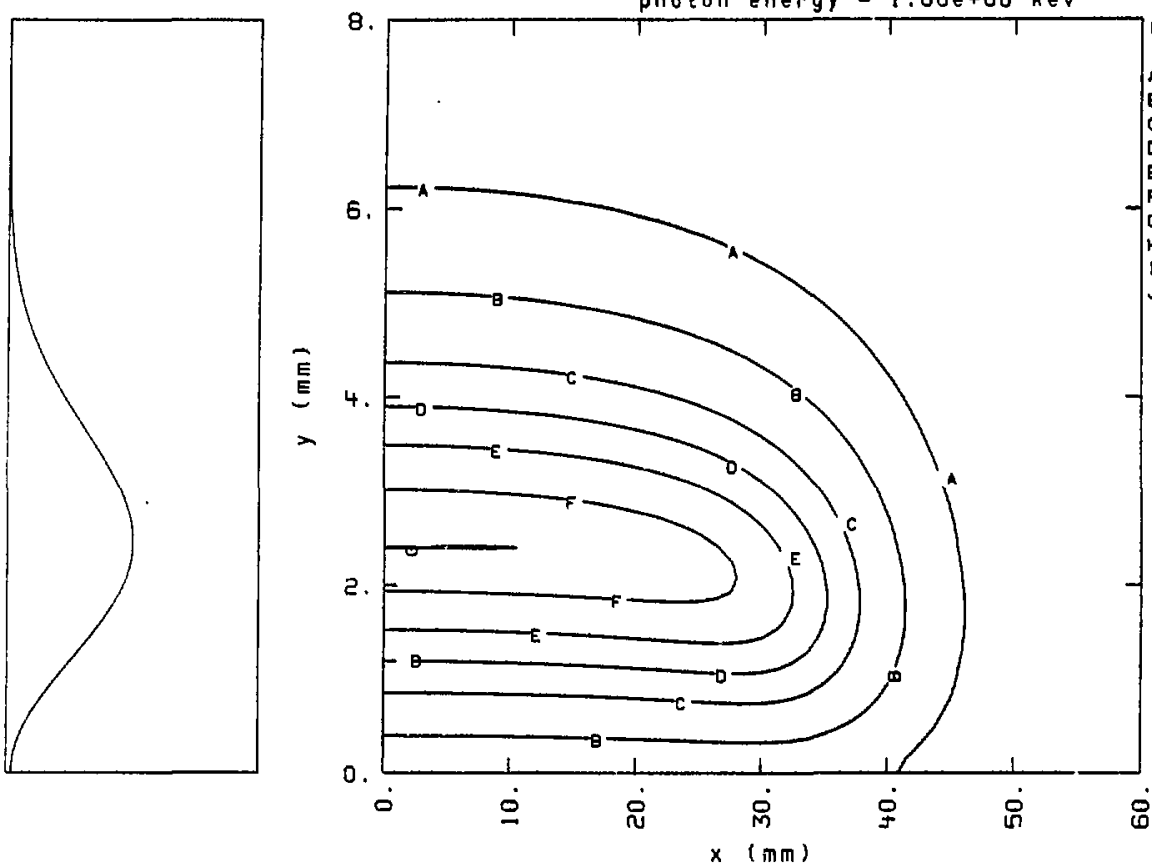

A 1.00-0e

o $1.00-01$

C $3.00,01$

D $5.00=0$

E 7.00001

F $9.00-01$

- 1.00000

H 1.10000

$1 \quad 1.40000$

2. 00000

$x$ (mm)

perpendicular polarization

normalized to

$z=4.24 \mathrm{e}+09$

at $x=0$.

$y=2.40 e+00$

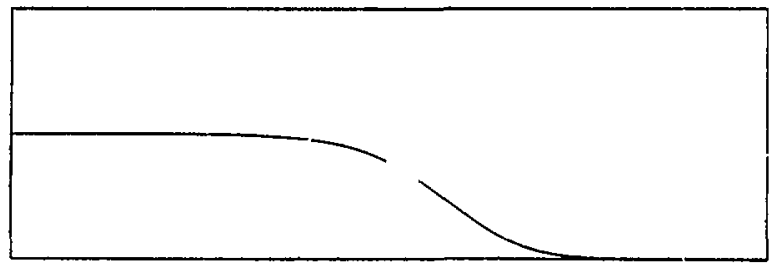

Fig. B3.4. 
wiggler: 30 poles; $B=13.0 \mathrm{hG} ; 1$ ambda $=12.85 \mathrm{~cm}$ $8.5 \mathrm{~m}$ from center of wiggler to mirror

electron energy $=1.80 \mathrm{e}+00 \mathrm{GeV}$ critical energy $=2.80 \mathrm{e}+00 \mathrm{hoV}$

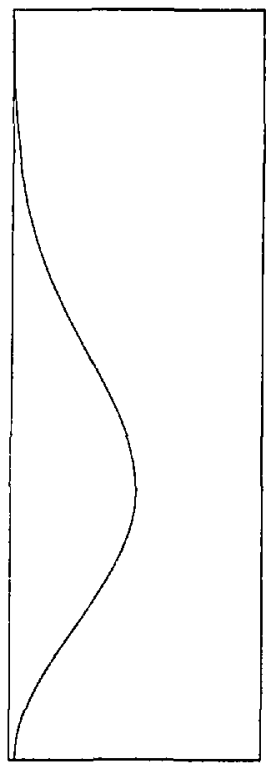

6 photon energy $=1.40 \mathrm{e}+00 \mathrm{keV}$

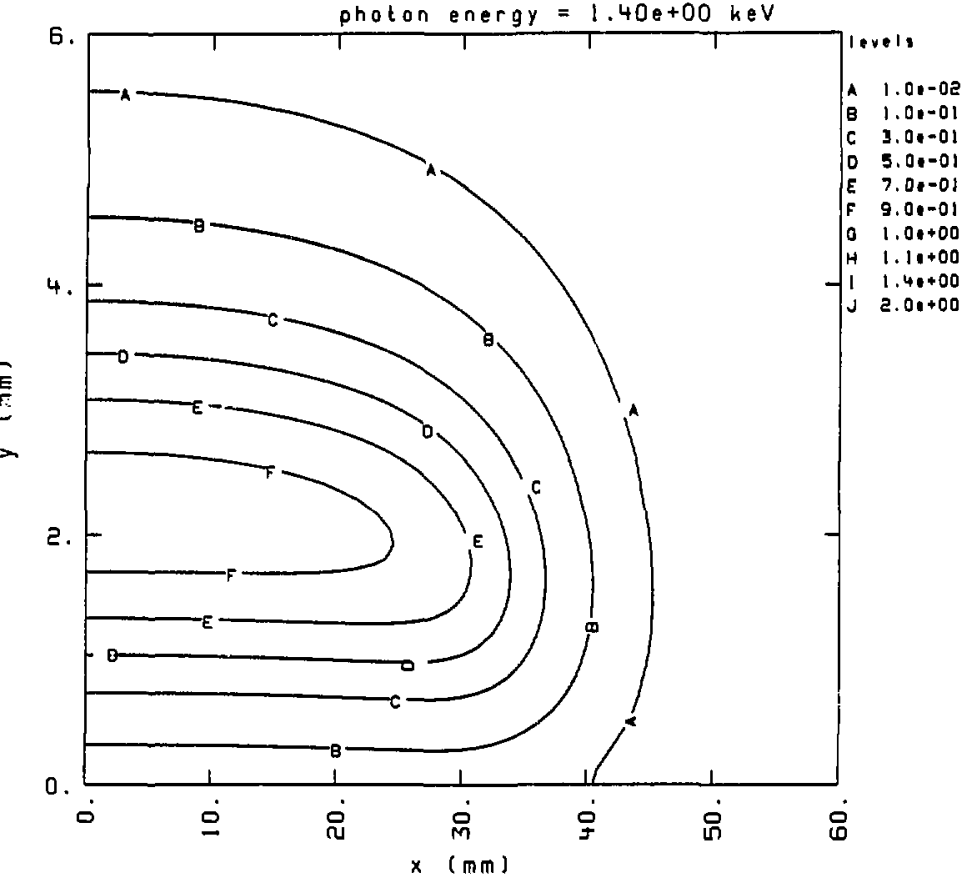

perpendicular polarization

normalized la

$z=4.15 e+09$

at $x=0$.

$y=2 \cdot 16 \mathrm{e}+00$

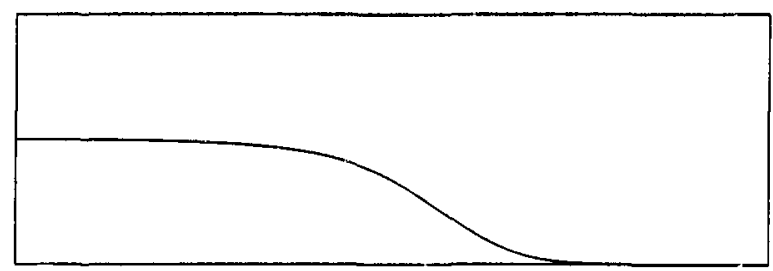

Fig. B3.5. 


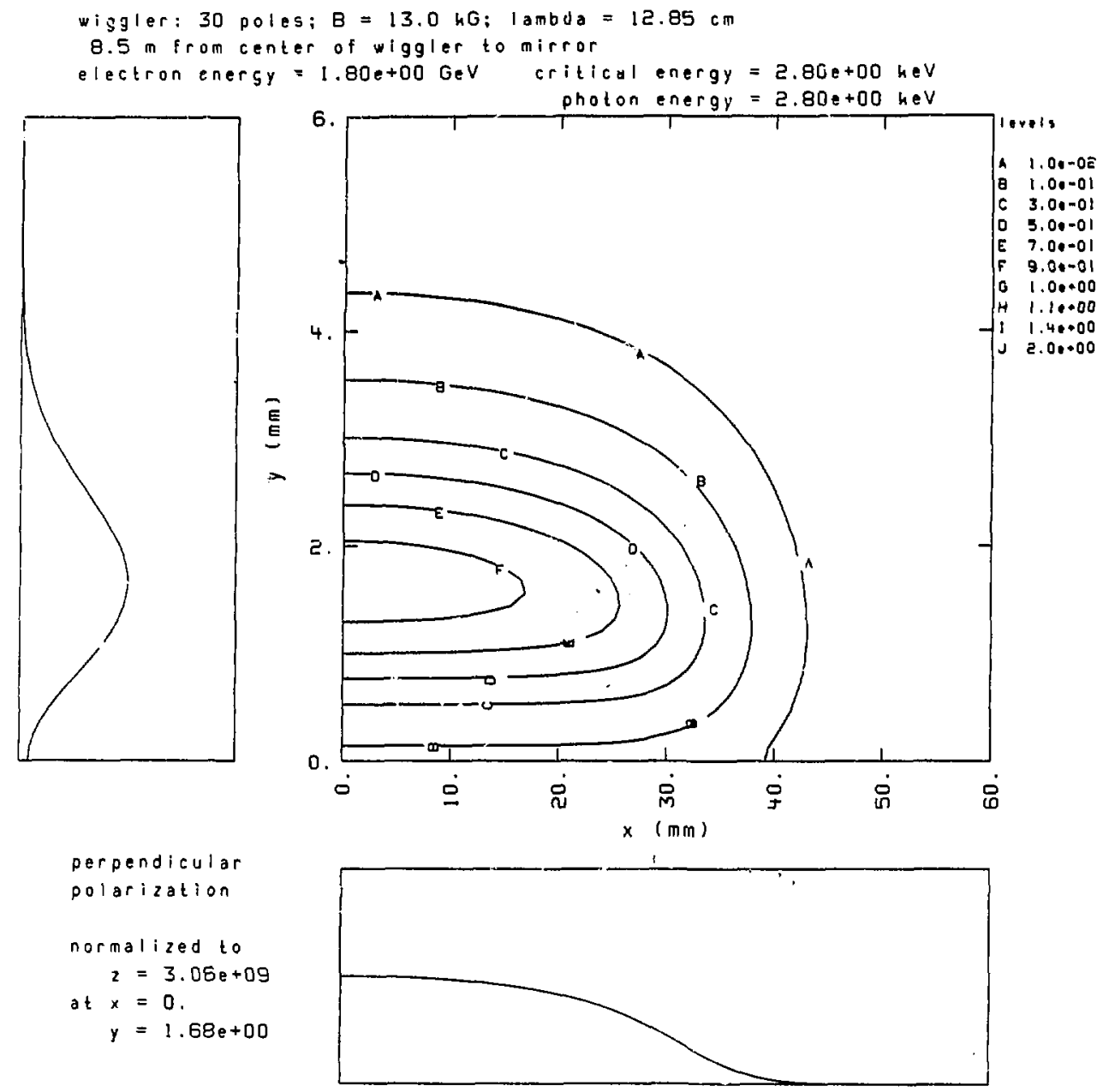

Fig. B3.6. 


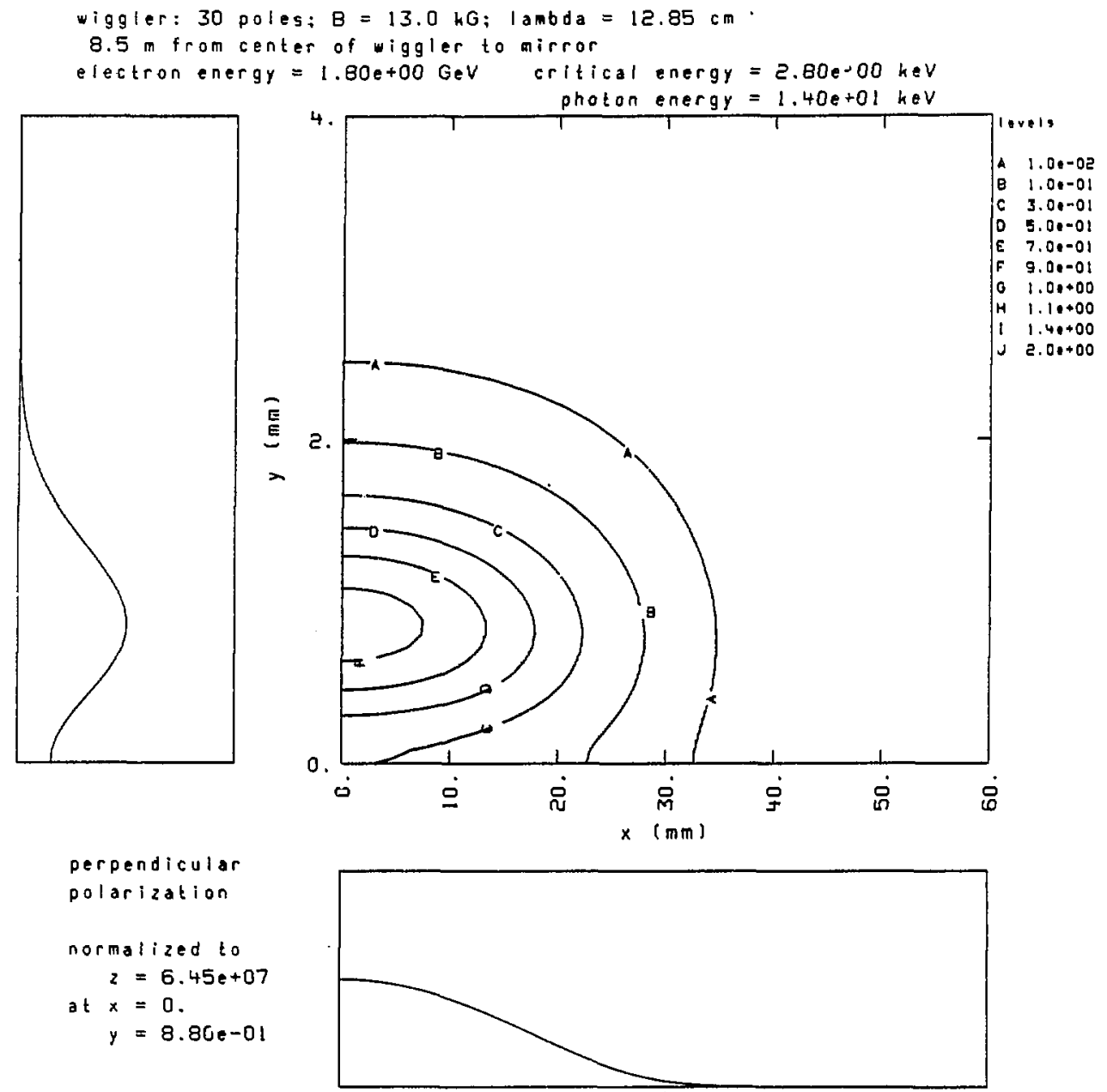

Fig. 83.7 
wiggler: 30 poles; $B=13.0 \mathrm{kG} ;$ lambda $=12.85 \mathrm{~cm}$

$8.5 \mathrm{~m}$ from center of wiggler to mirror

electron energy $=3.00 \mathrm{e}+00 \mathrm{GeV}$ criticul energy $\times 7.78 \mathrm{e}+00 \mathrm{keV}$
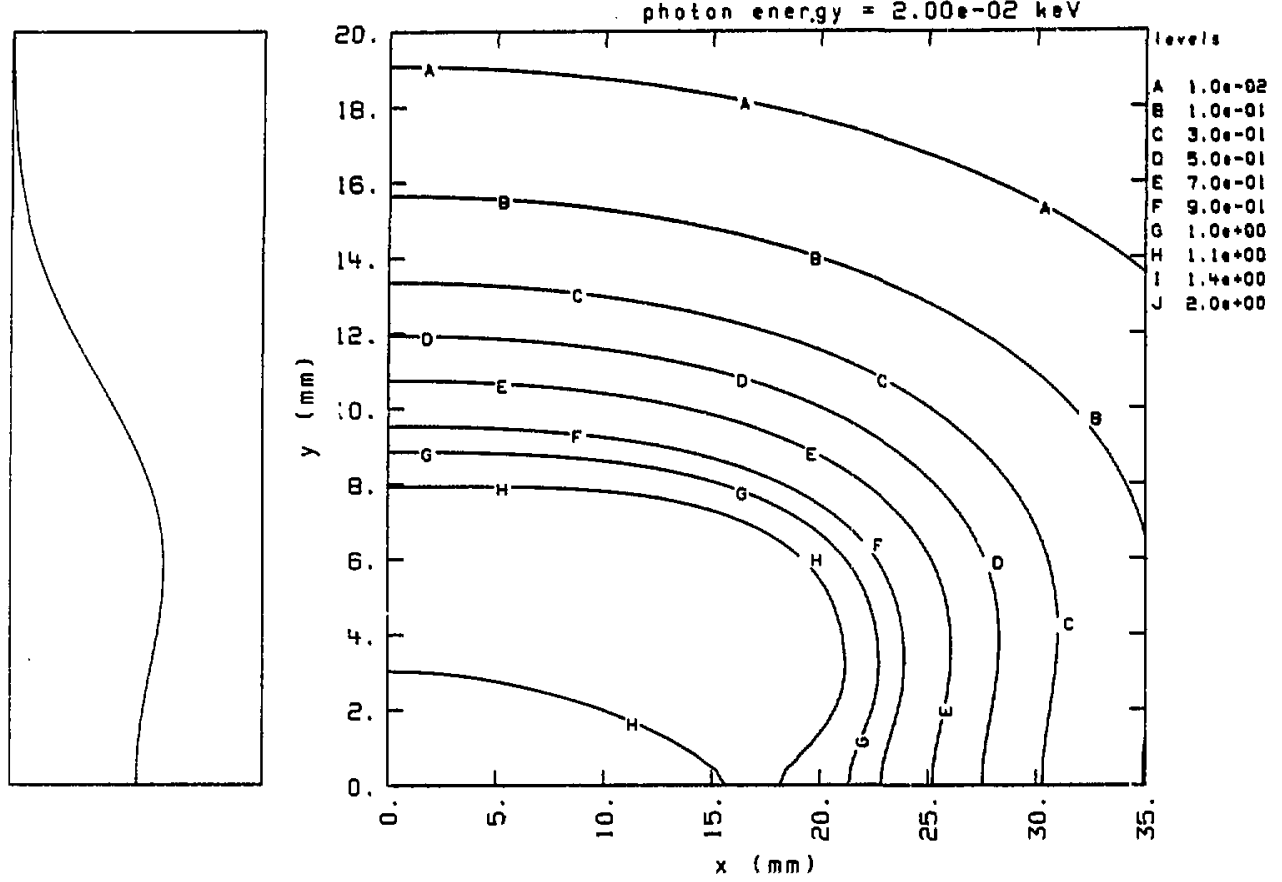

normalized to

$z=2.73 e+09$

at $x=0$.

$y=0$.

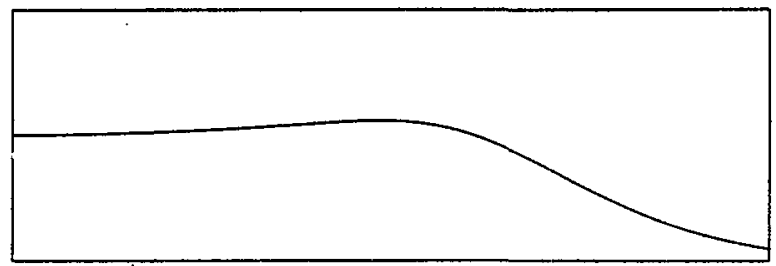

Fig. B4.1 
wiggler: 30 poles; $B=13.0 \mathrm{kG}: \operatorname{lambda}=12.85 \mathrm{~cm}$

$8.5 \mathrm{~m}$ from center of wiggler to mirror

electron energy $=3.00 \mathrm{e}+00 \mathrm{GeV}$ critical energy $=7.78 \mathrm{e}+00 \mathrm{keV}$
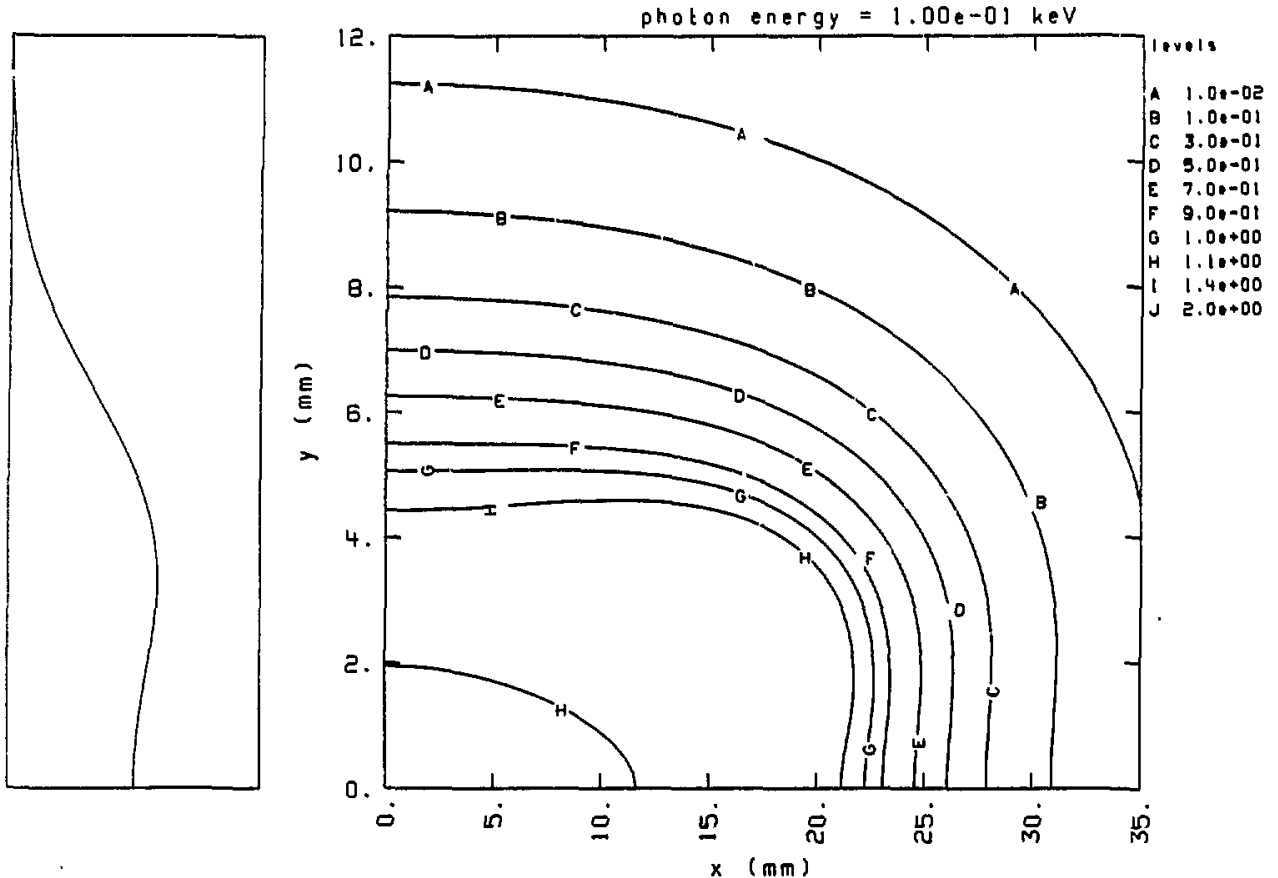

$$
\begin{aligned}
\text { normalized to } & \text { tize } \\
z & =8.05 e+09 \\
\text { at } x & =0 . \\
y & =0 .
\end{aligned}
$$

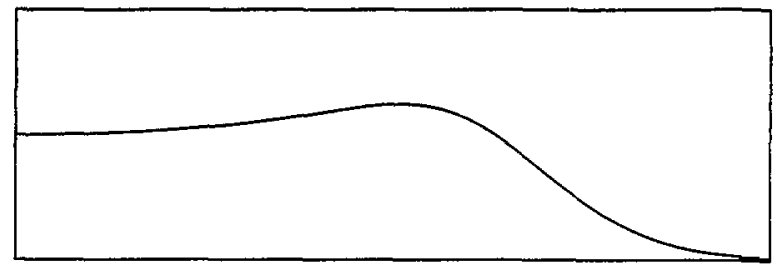

Fig. 34.2 


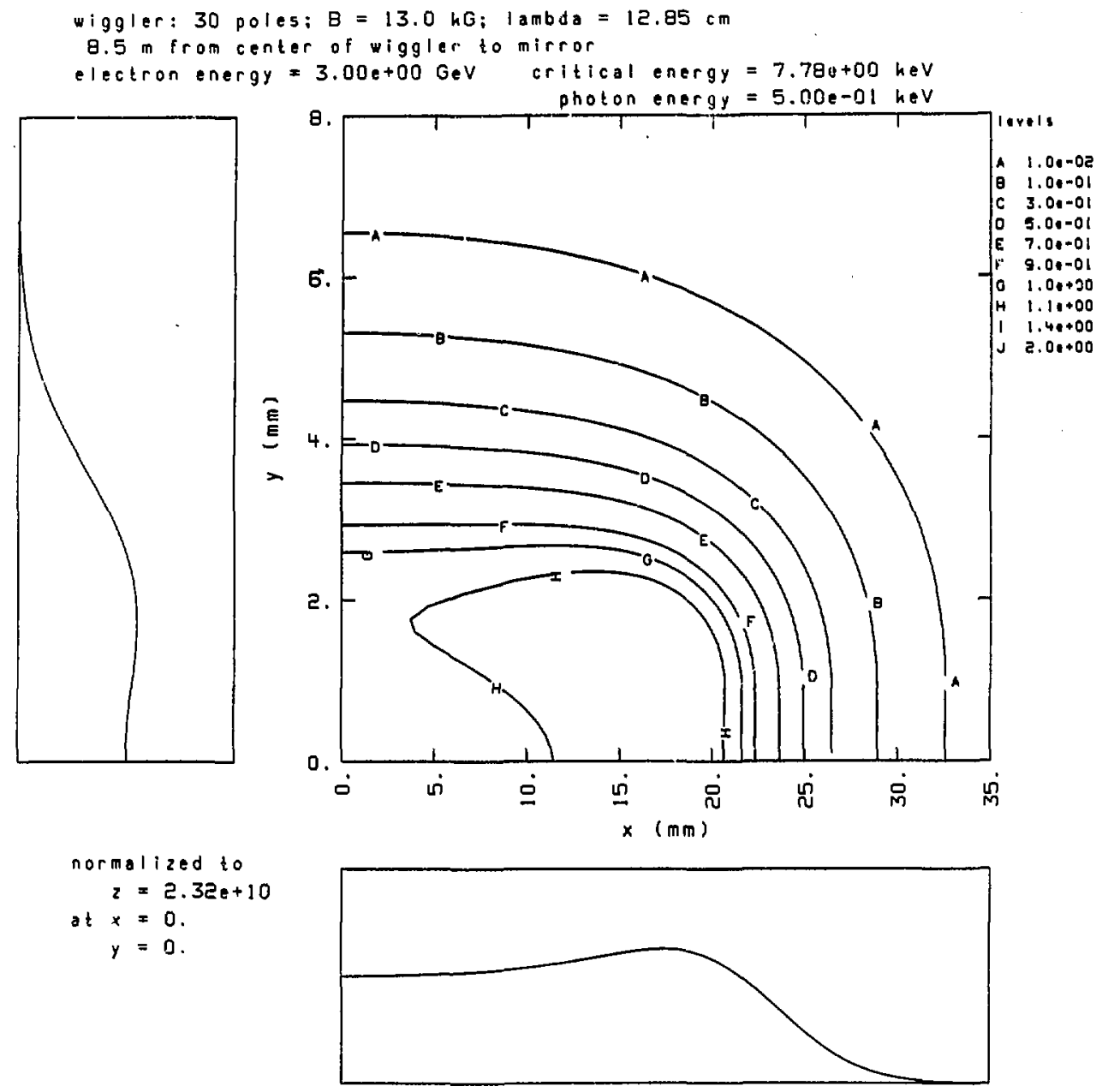

Fig. B4.3 


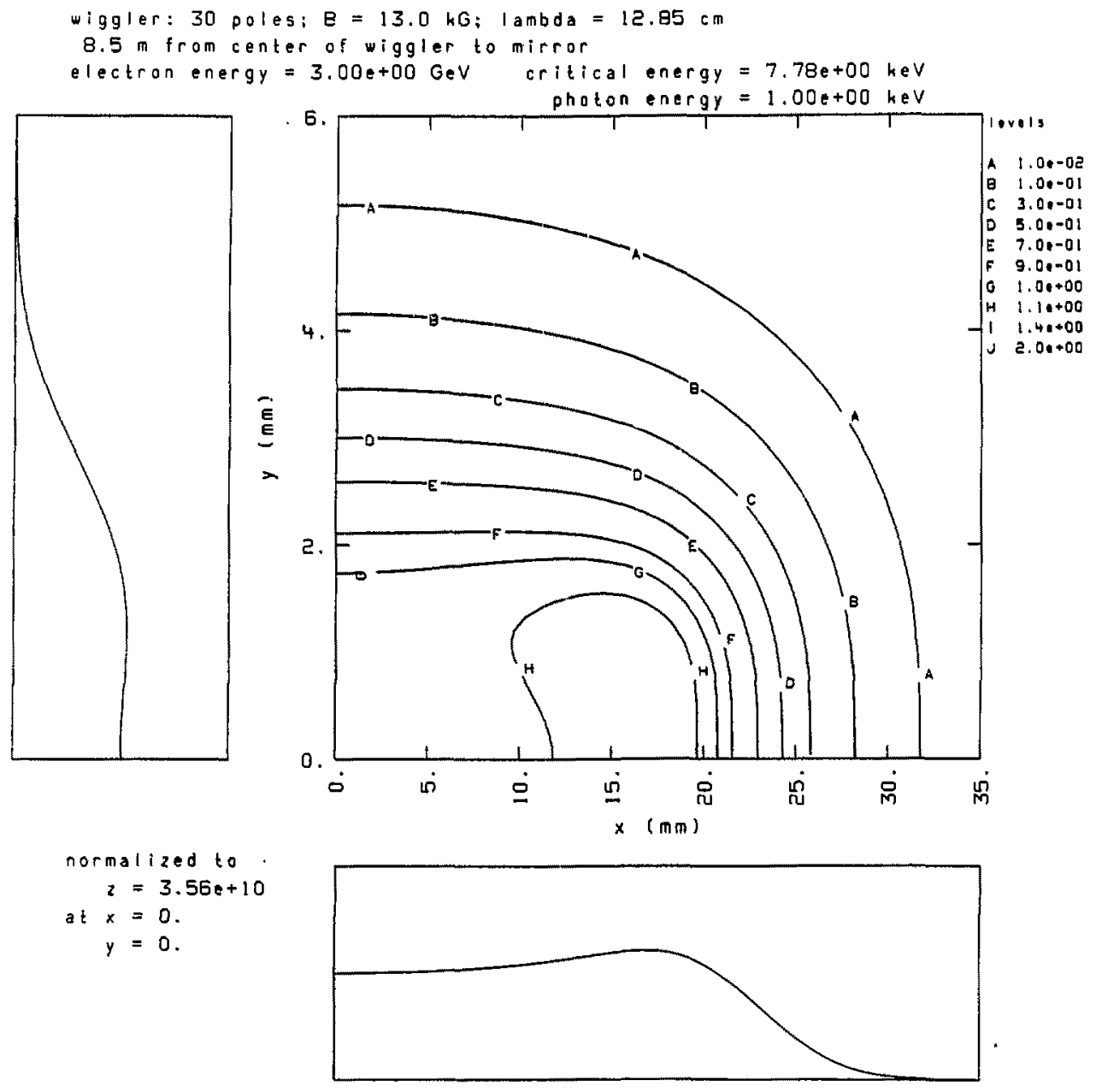

Fig. B4.4. 
wiggler: 30 poles; $B=13.0 \mathrm{kG}: 1 \mathrm{ambda}=12.05 \mathrm{~cm}$

$8.5 \mathrm{~m}$ from center of wiggler to mirror

electron energy $=3.00 \mathrm{e}+00 \mathrm{GeV}$ critical energy $=7.78 \mathrm{e}+00 \mathrm{keV}$

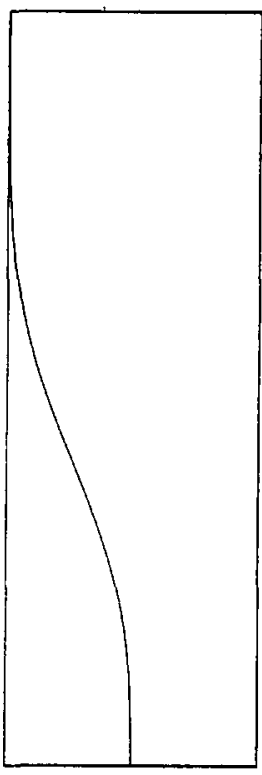

photon energy $=3.89 e+00 \mathrm{keV}$

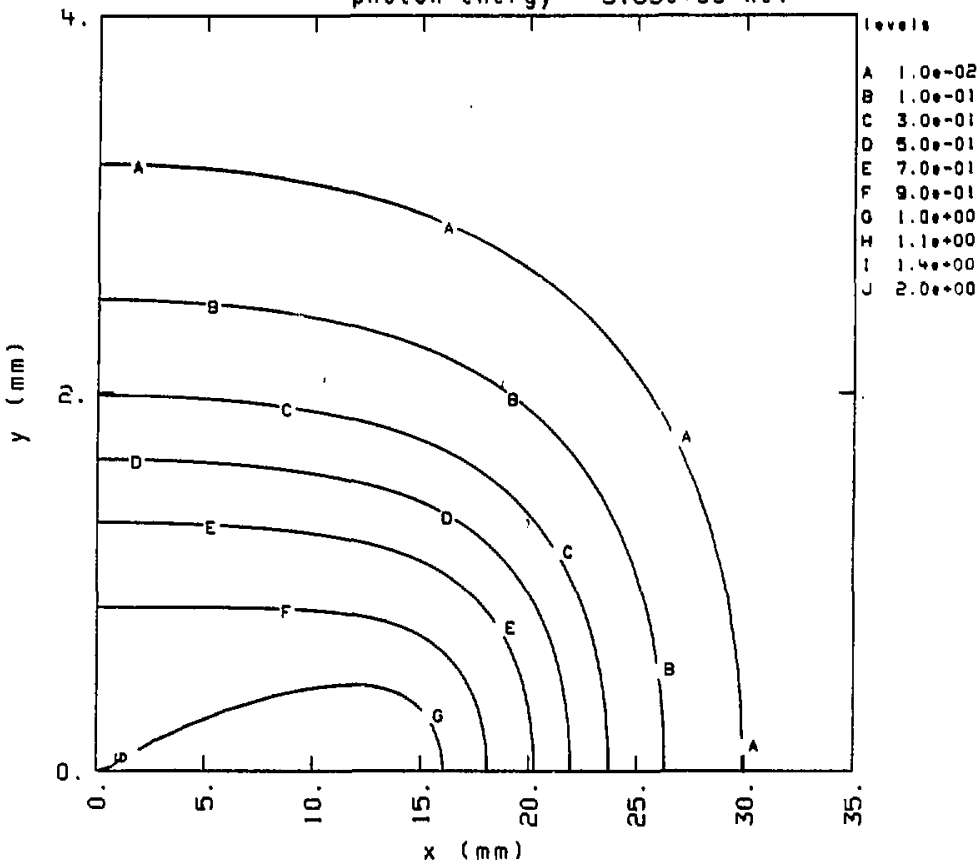

$$
\begin{aligned}
\text { normalized to } & \begin{aligned}
z & =6.79 \mathrm{e}+10 \\
\text { at } x & =0 . \\
y & =0 .
\end{aligned}
\end{aligned}
$$

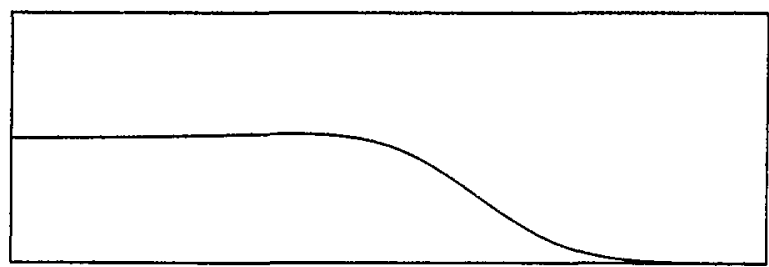

Fig. B4.5. 


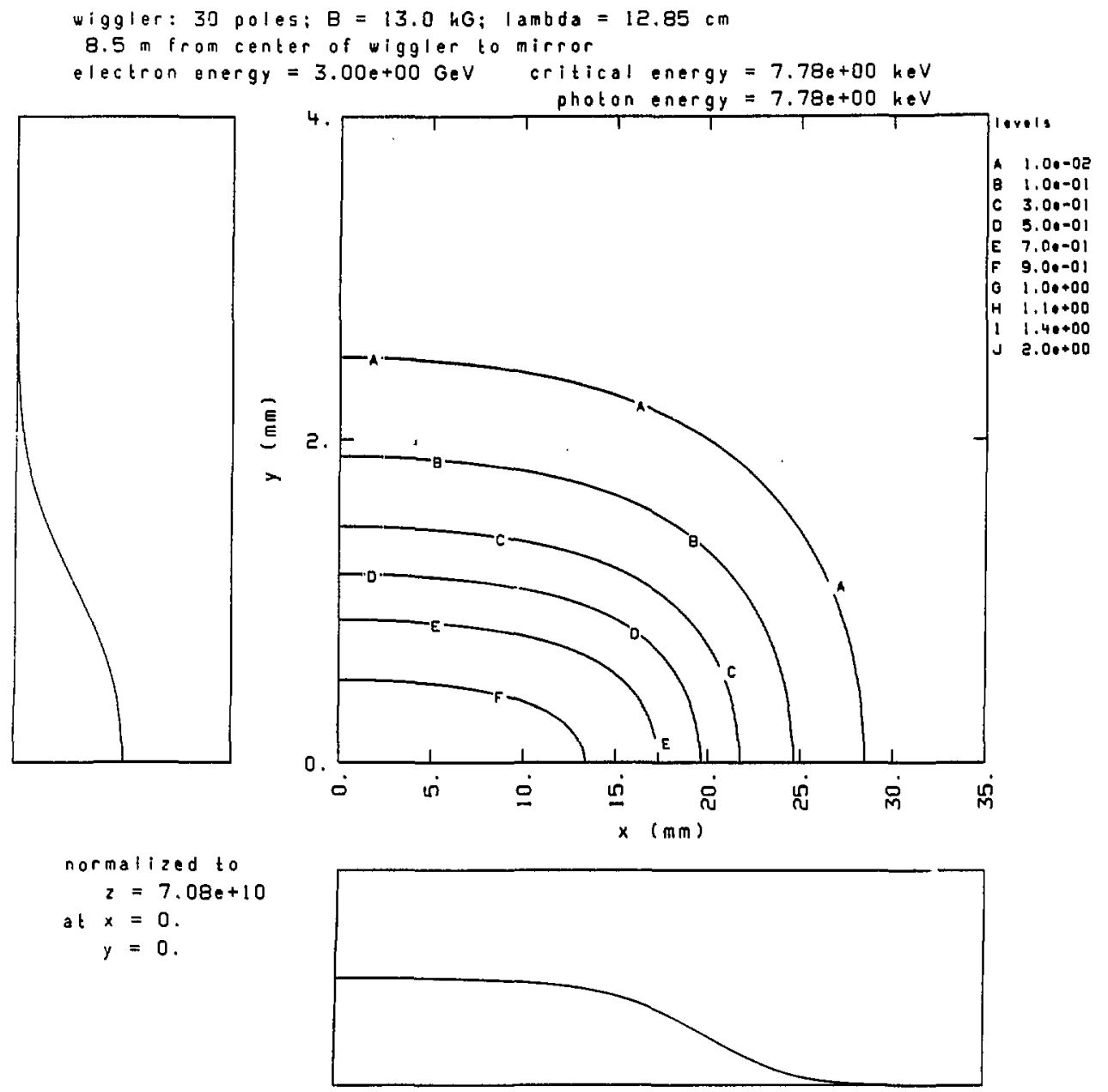

Fig. B4.6. 


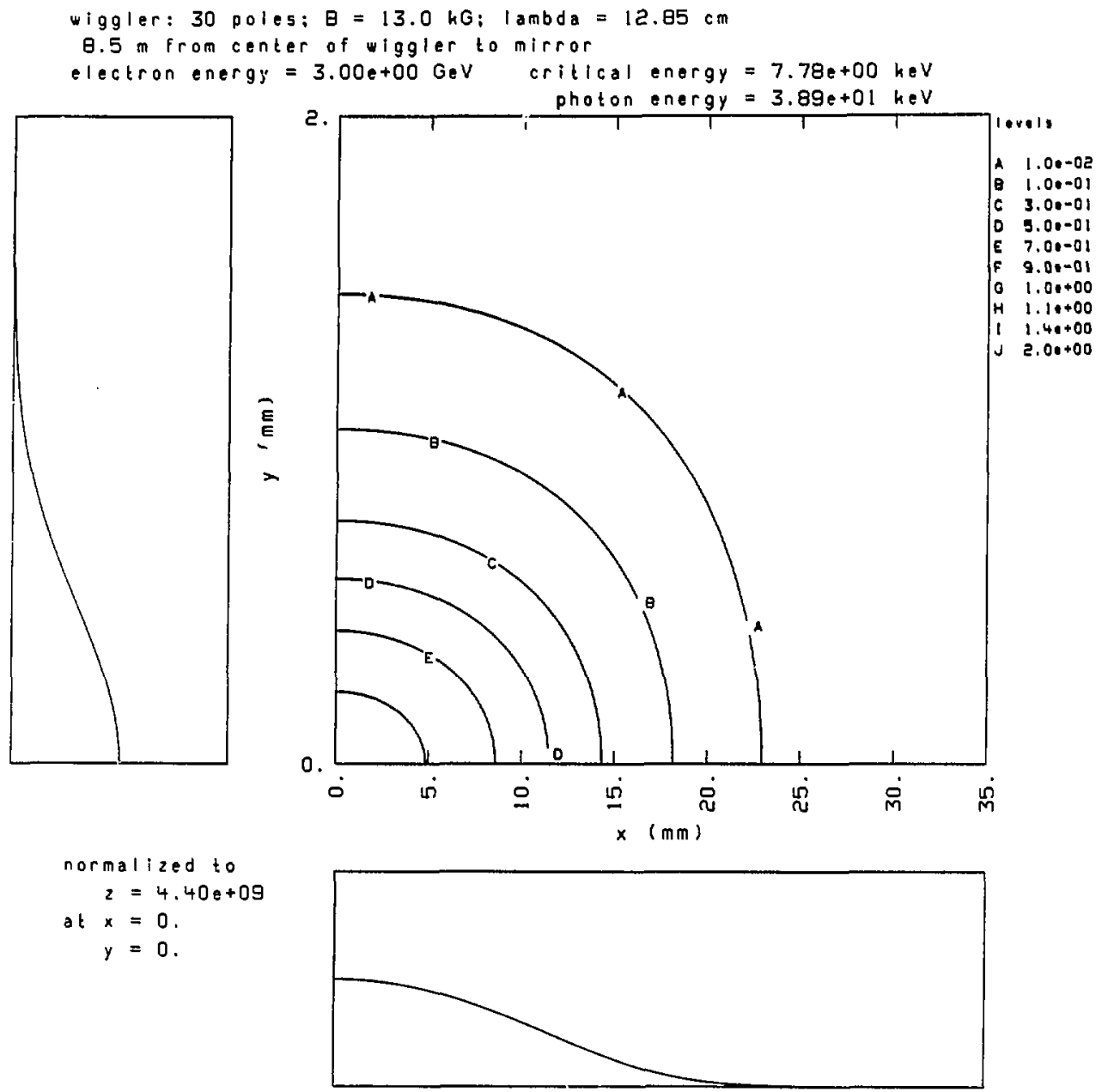

Fig. B4.7. 

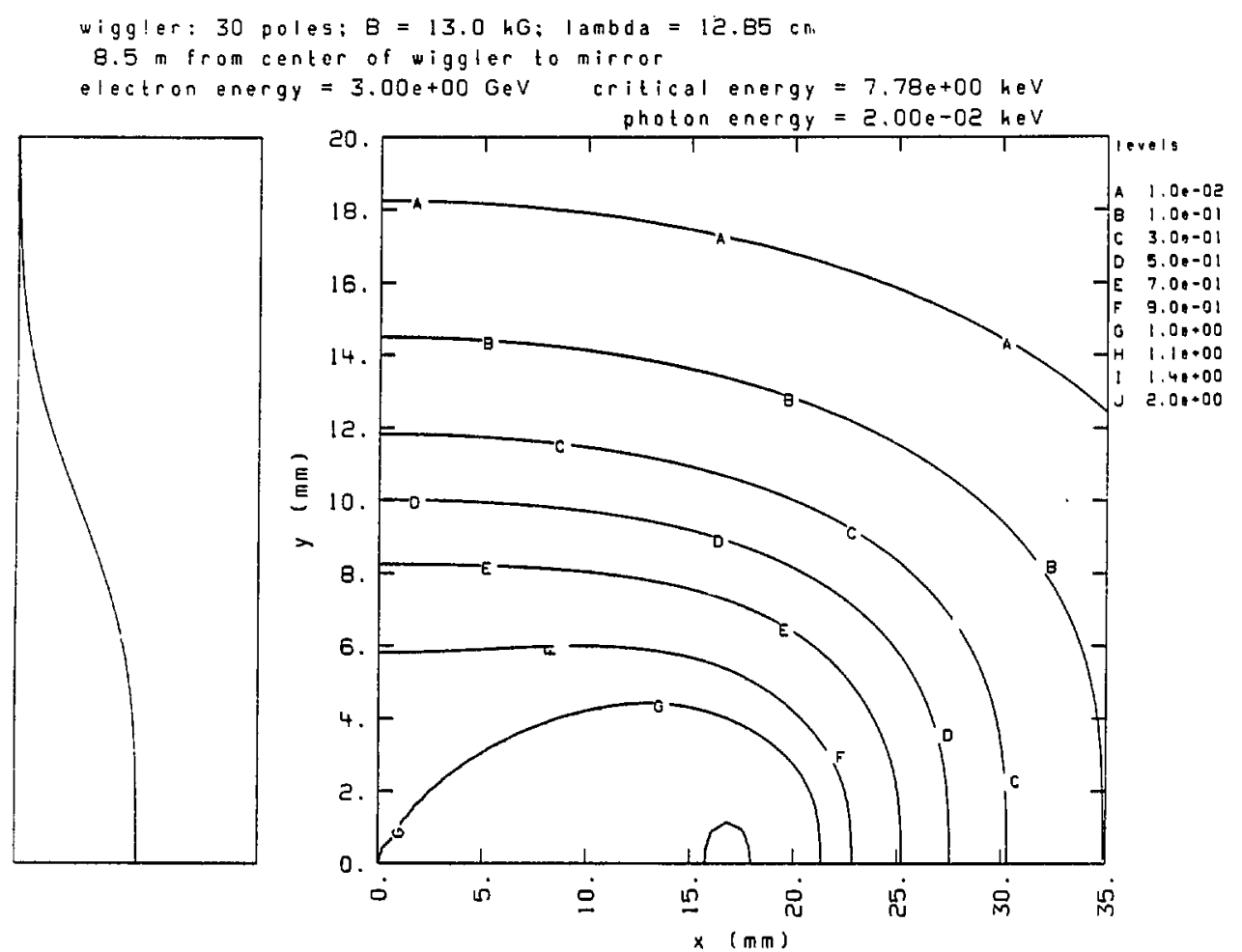

parallel

polarization

normalized to

$z=2.72 e+09$

at $x=0$.

$y=0$.

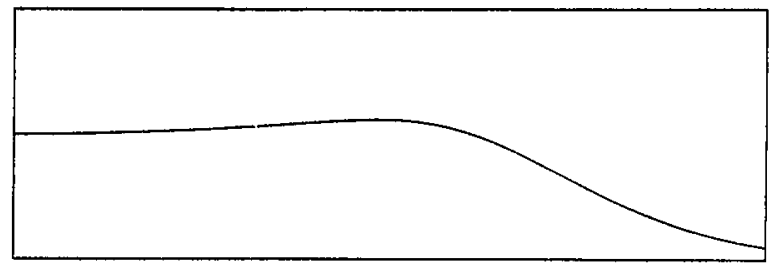

Fig. B5. 1 . 


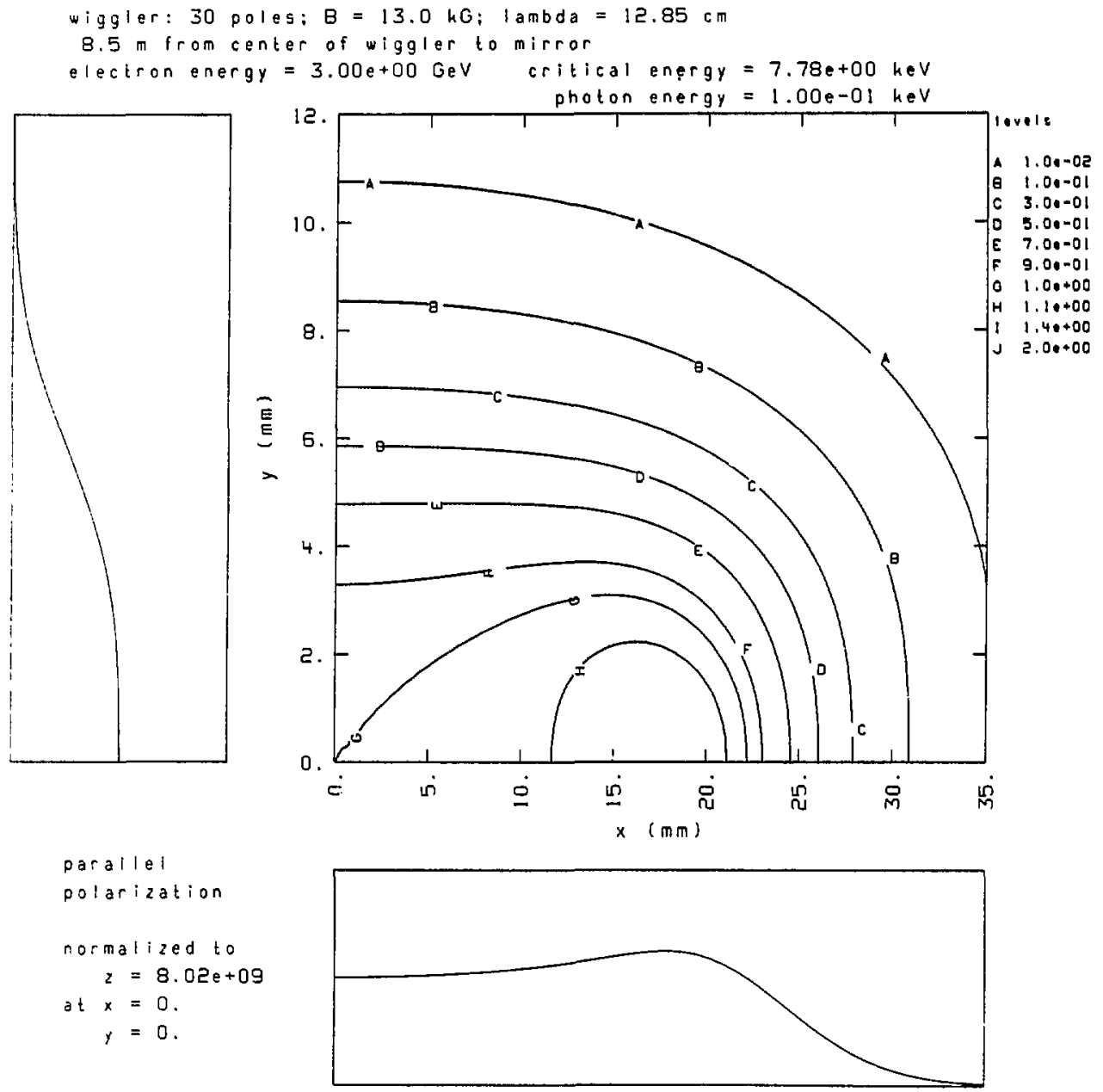

Fig. B5.2. 


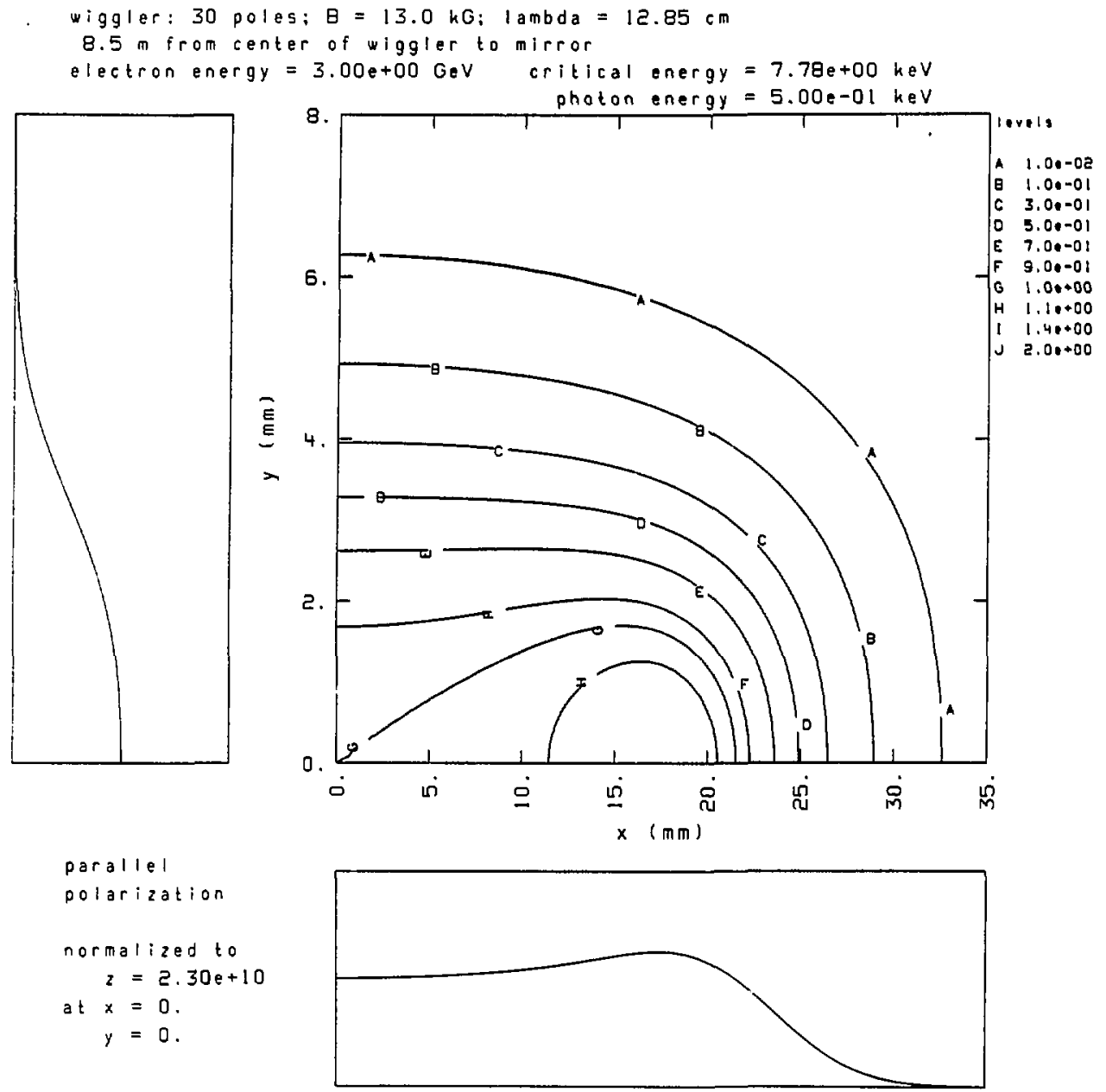

Fig. B5.3. 
wiggler: 30 poles; $B=13.0 \mathrm{hG}$; lambda $=12.85 \mathrm{~cm}$

$8.5 \mathrm{~m}$ from center of wiggler to mirror

electron energy $=3.00 \mathrm{e}+00 \mathrm{GeV}$ critical energy $=7.78 \mathrm{e}+00 \mathrm{heV}$
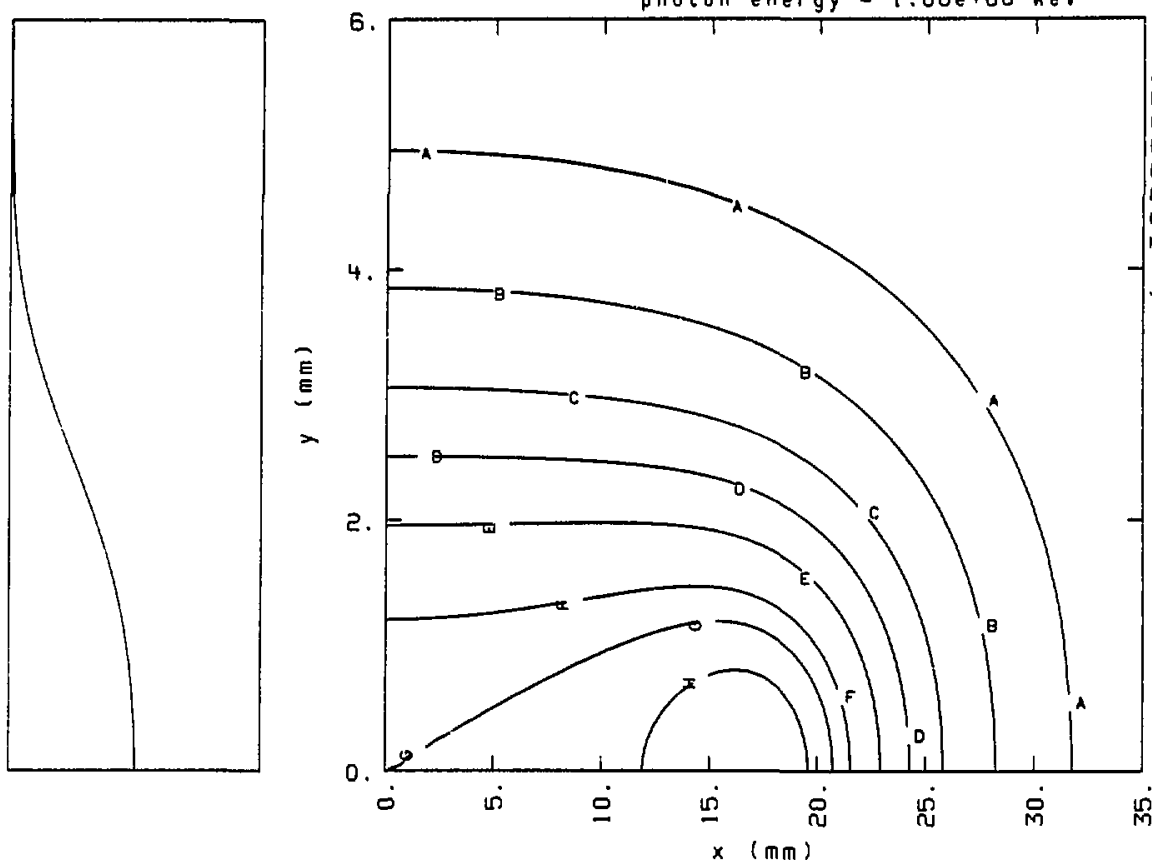

parallel

polarization

normalized to

$z=3.32 \mathrm{e}+10$

at $x=0$.

$y=0$.

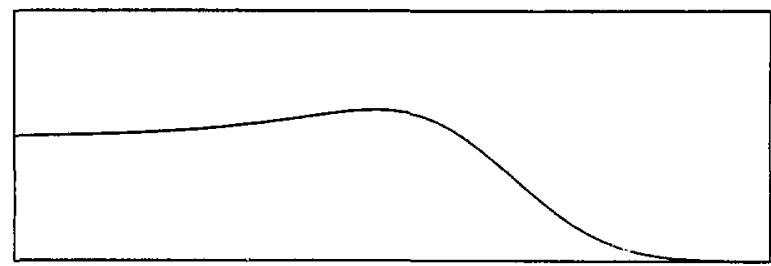

Fig. B5.4. 


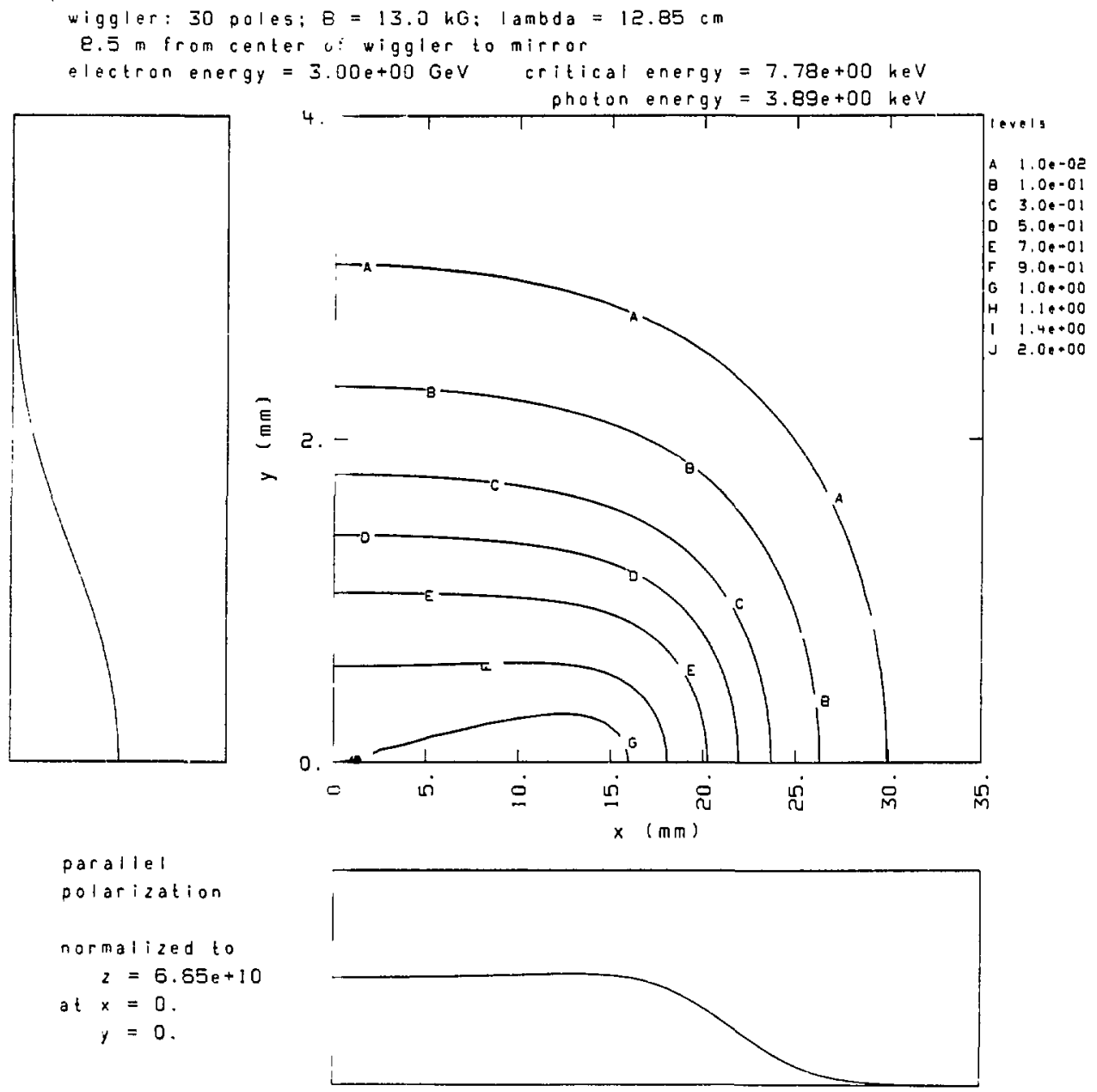

Fig. B5.5. 


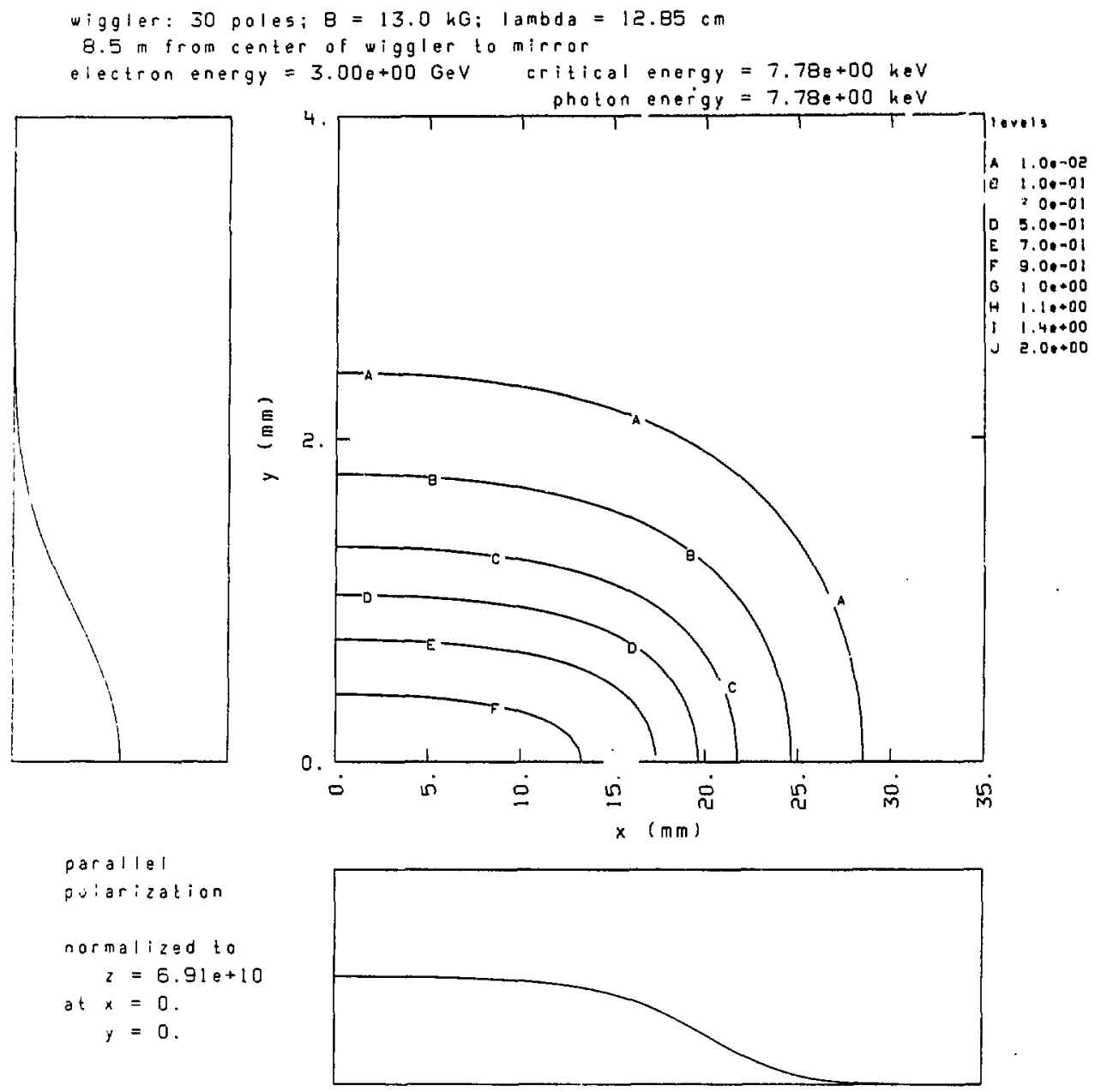

Fig. B5.6. 
wiggler: 30 poles; $8=13.0 \mathrm{kG} ;$ lambda $=12.85 \mathrm{~cm}$

$8.5 \mathrm{~m}$ from center of wiggler to mirror

electron energy $=3.00 \mathrm{e}+00 \mathrm{GeV}$ critical energy $=7.78 \mathrm{e}+00 \mathrm{keV}$
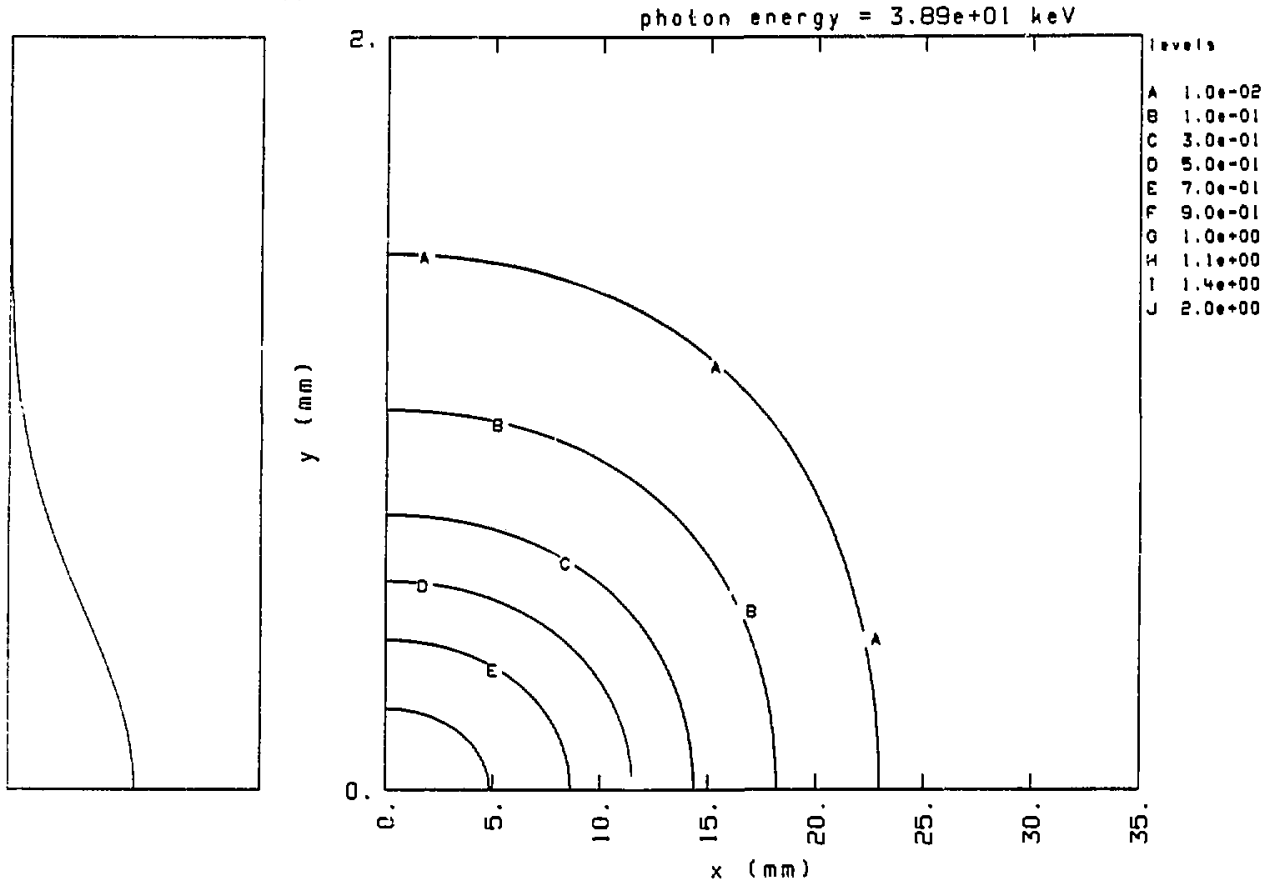

$$
\begin{aligned}
& \text { parallel } \\
& \text { polarization } \\
& \text { normalized to } \\
& z=4.31 e+09 \\
& \text { at } x=0 \text {. } \\
& y=0 \text {. }
\end{aligned}
$$

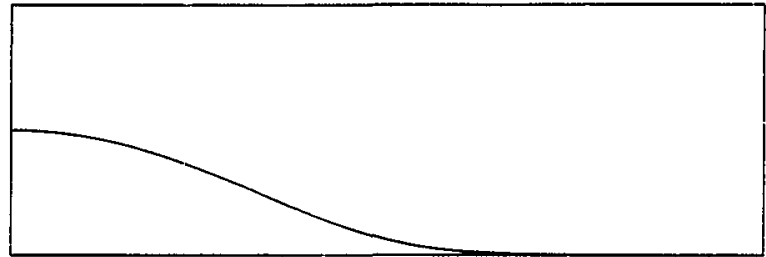

Fig. B5.7. 
wiggler: 30 poles: $B=13.0 \mathrm{hG} ; 1$ ambda $=12.85 \mathrm{~cm}$

$8.5 \mathrm{~m}$ from center of wiggler to mirror

electron energy $=3.00 \mathrm{e}+00 \mathrm{GeV}$ critical energy $=7.78 \mathrm{e}+00 \mathrm{heV}$
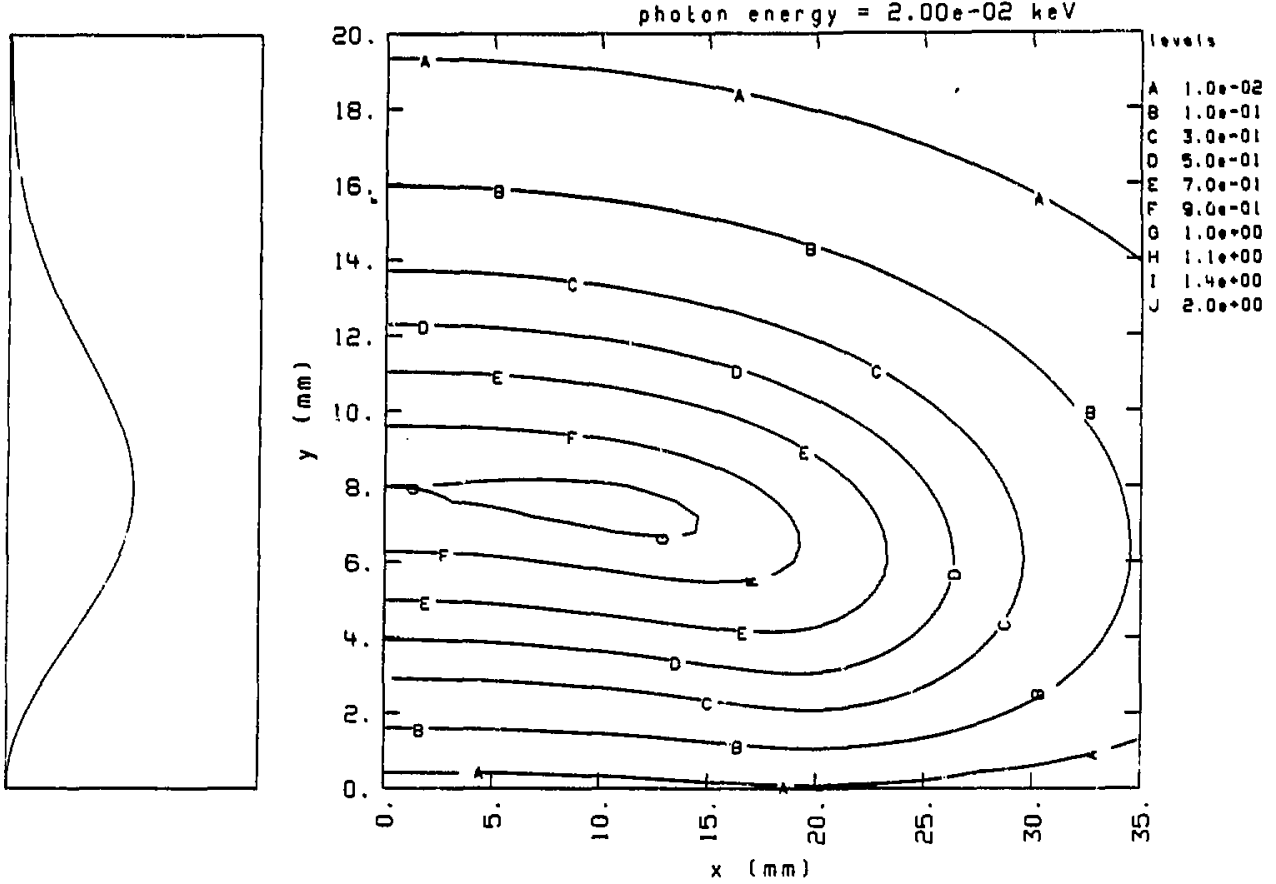

perpendicular polarization

normalized to

$z=1.04 \mathrm{e}+09$

at $x=0$.

$y=8.00 e+C 0$

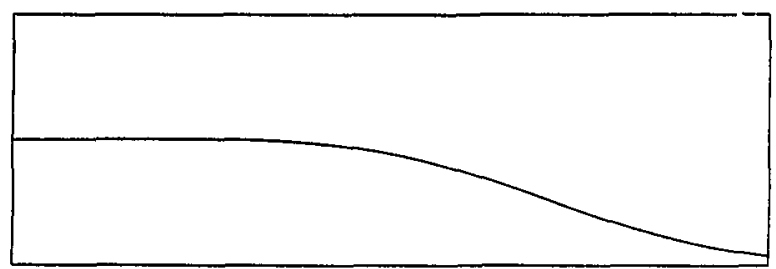

Fig. B6.1. 


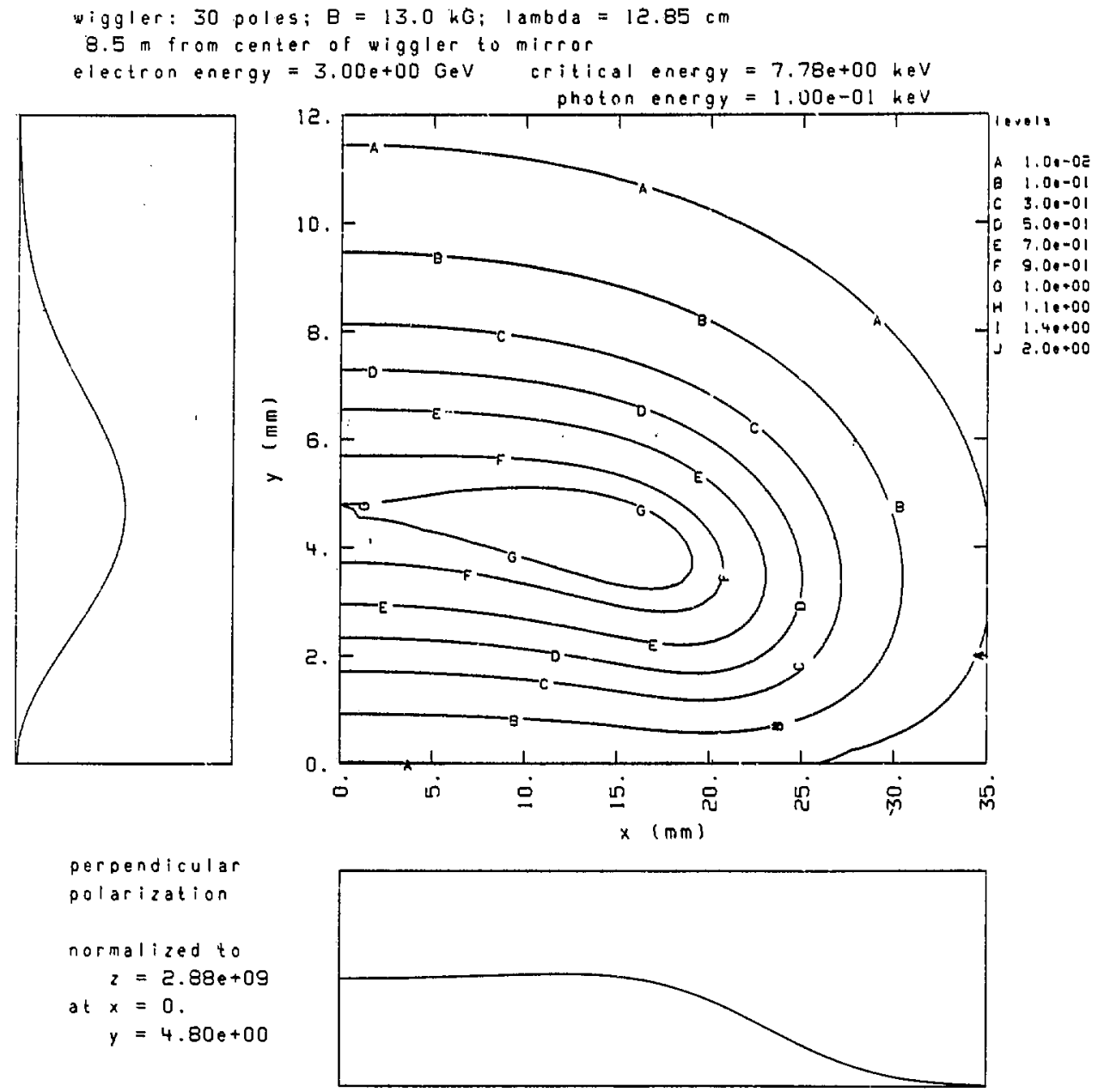

Fig. B6,2. 
wiggler: 30 poles: $\theta=13.0 \mathrm{hG:}$ lambda $=12.85 \mathrm{~cm}$

$8.5 \mathrm{~m}$ from center of wiggler to mirror

electron energy $=3.00 \mathrm{e}+00 \mathrm{GeV}$ critieal energy $=7.780+00 \mathrm{keV}$
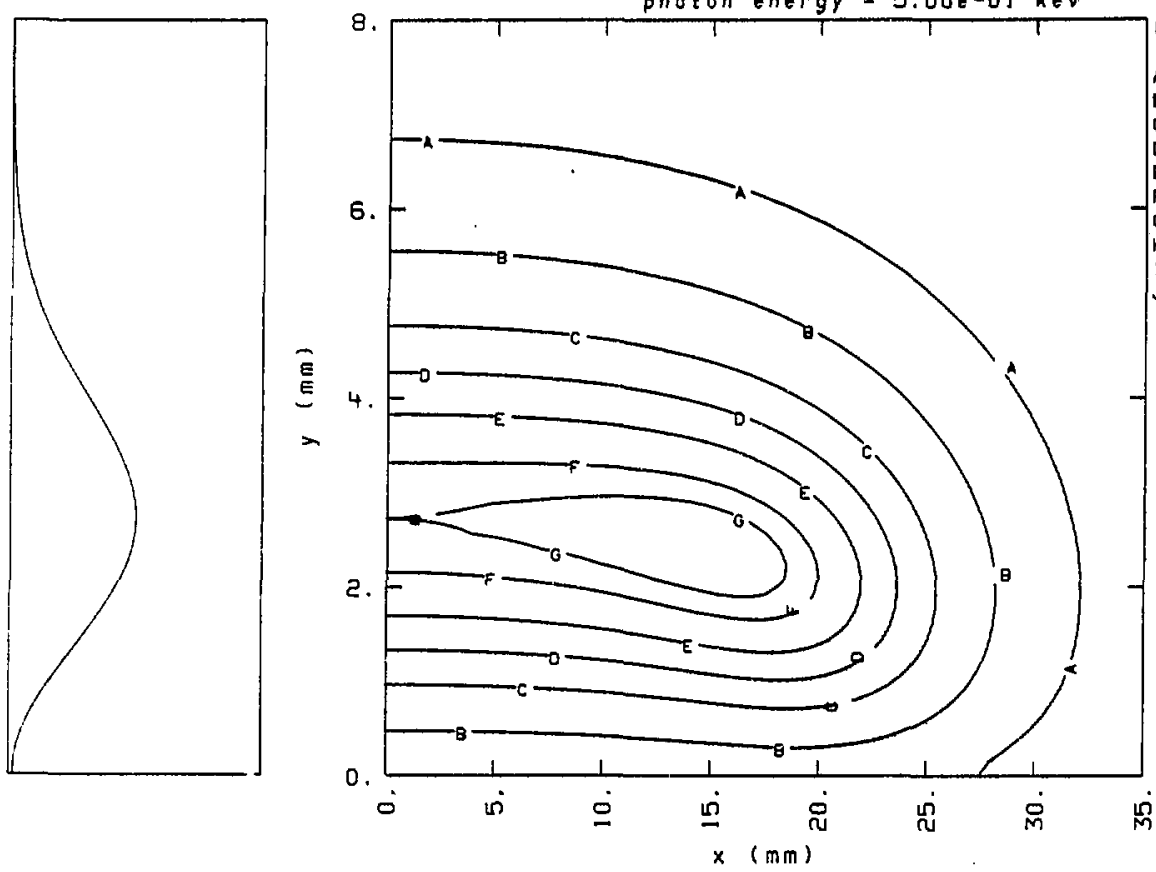

perpondicular polarization

normalized to

$z=6.96 \mathrm{e}+09$

at $x=0$.

$y=2.72 e+00$

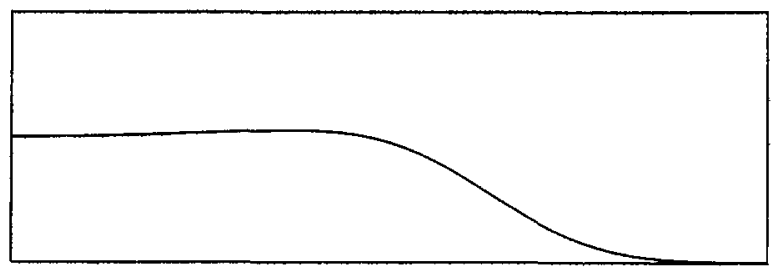

Fig. B6.3. 


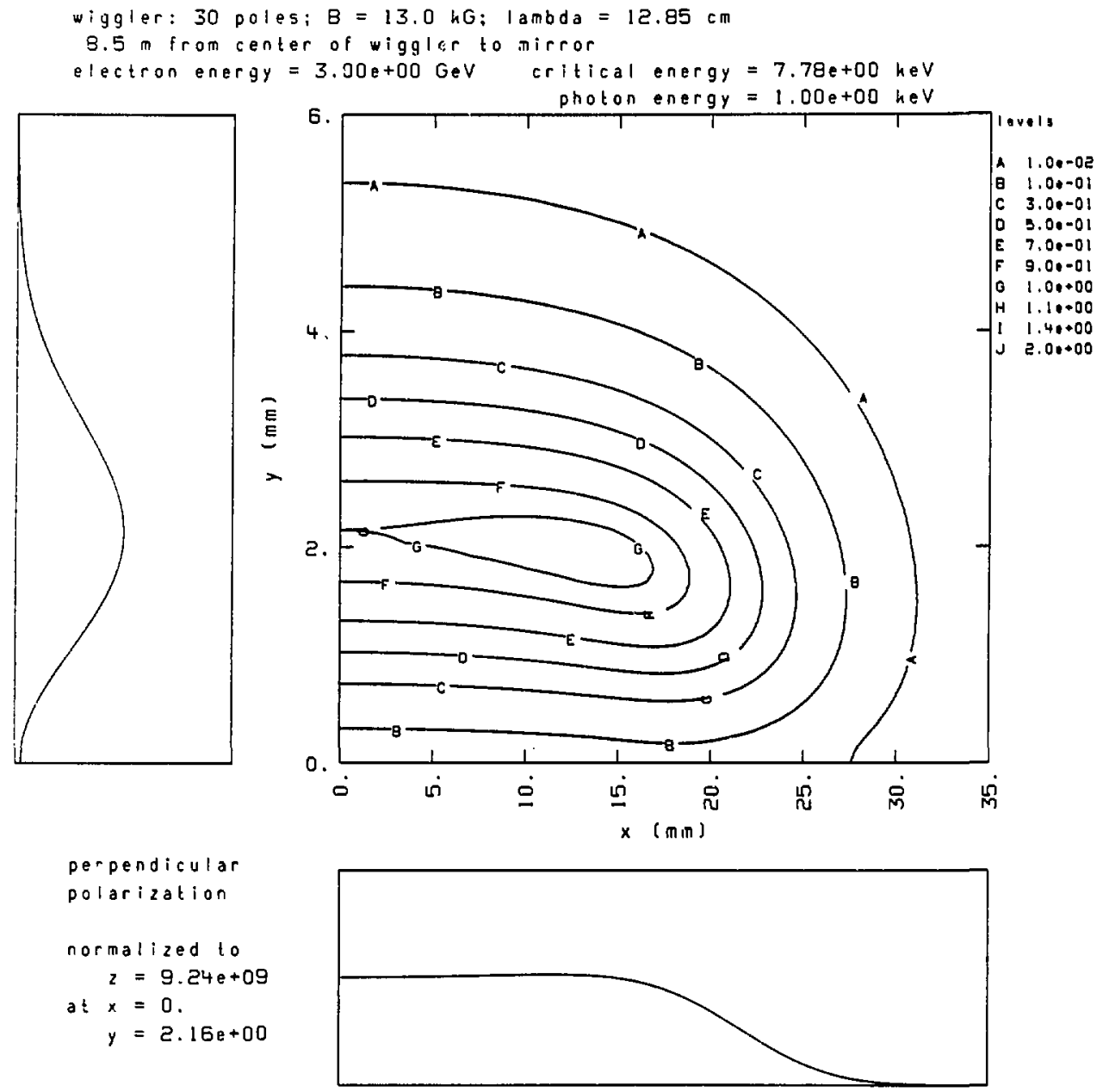

Fig. B6.4. 


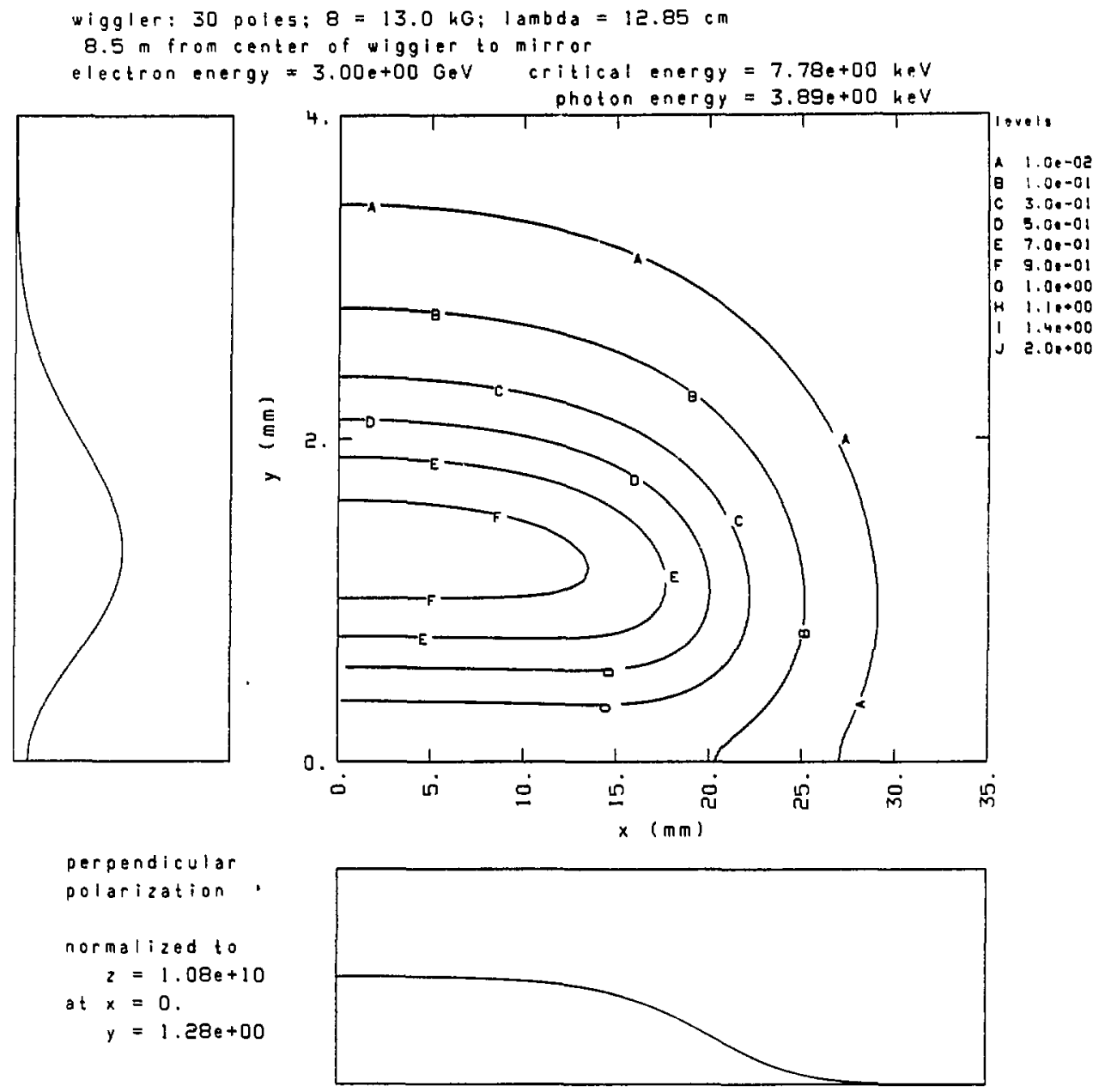

Fig. B6.5. 


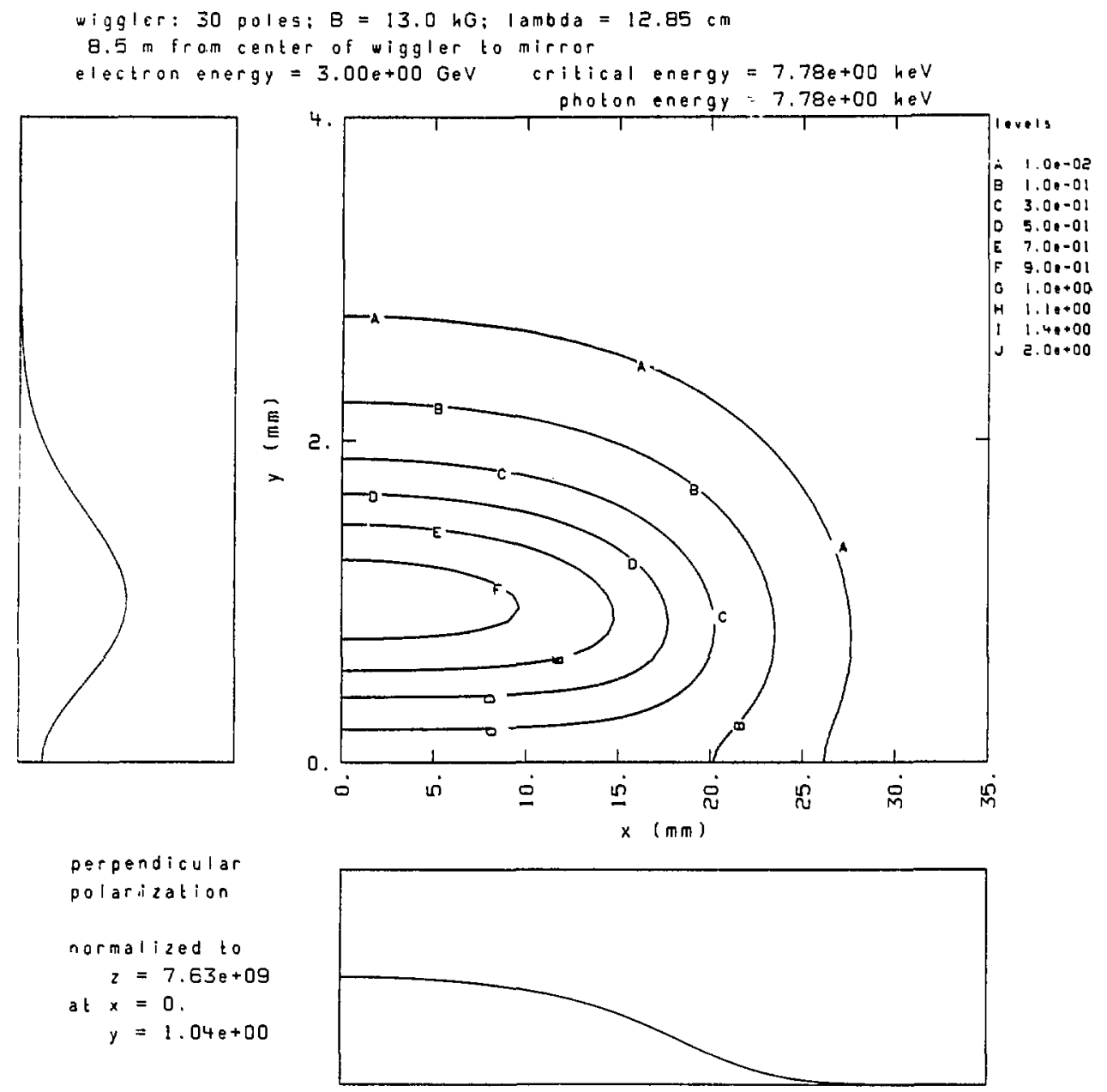

Fig. B6.6. 


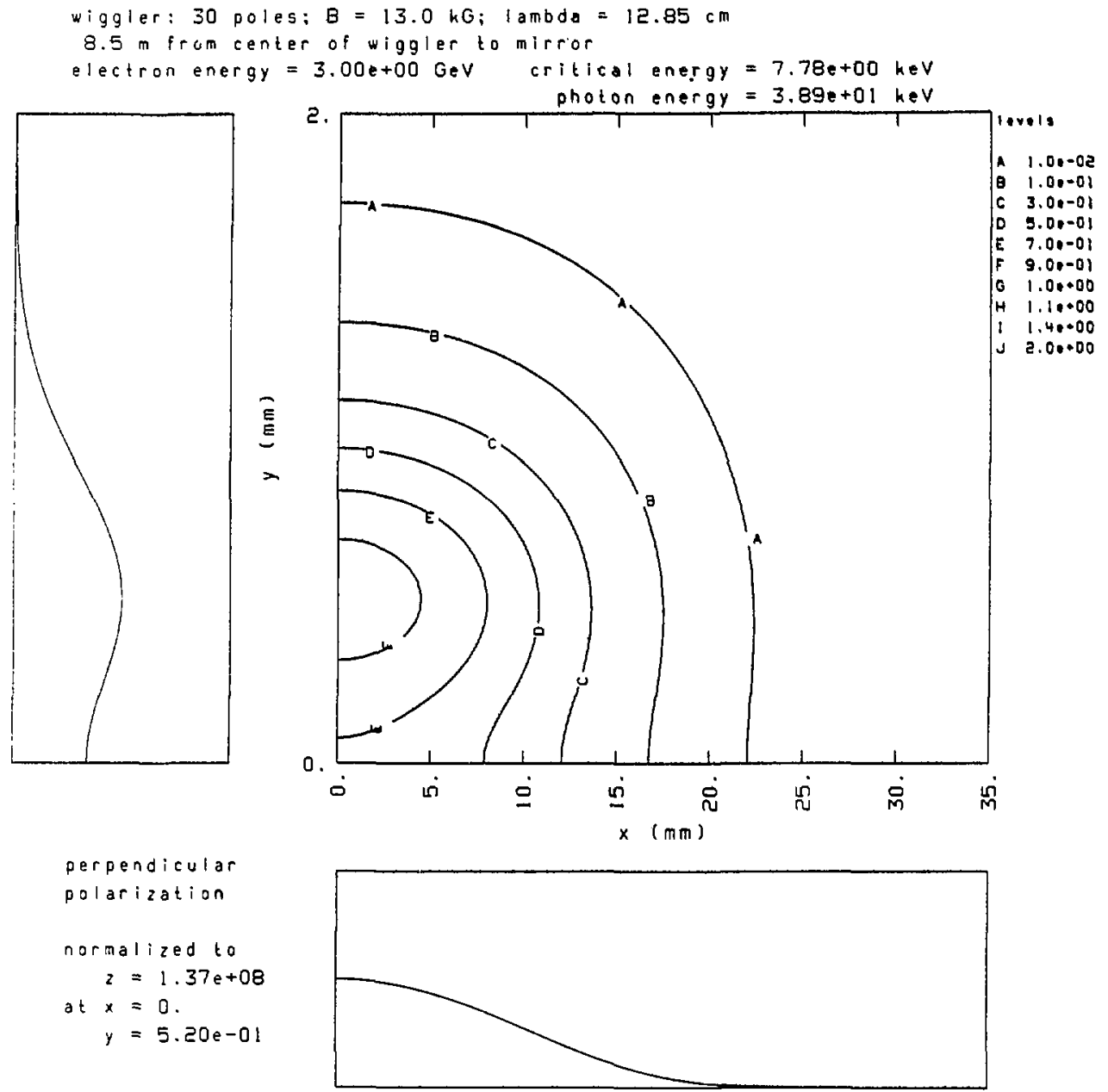

Fig. B6.7. 


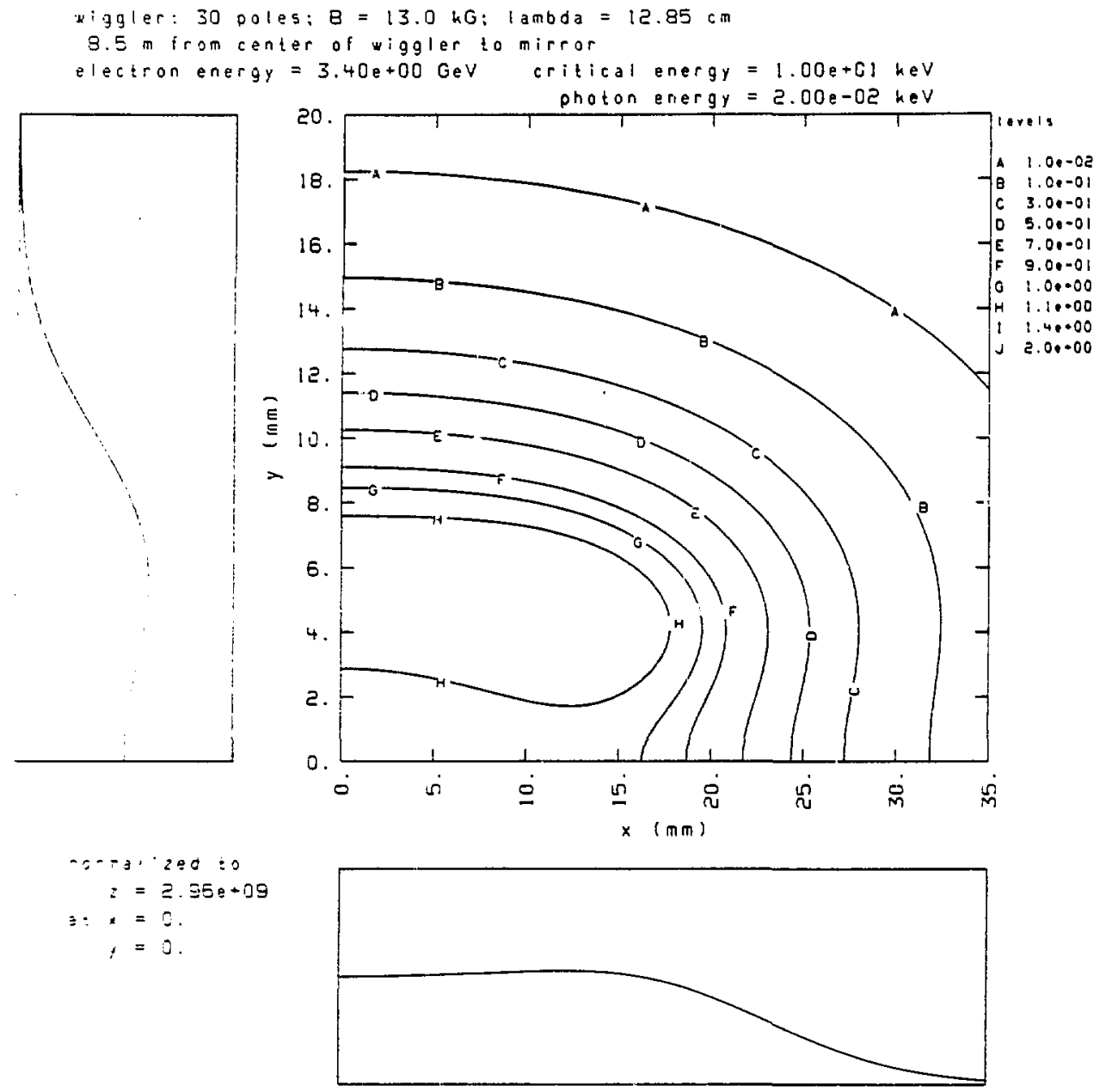

Fig. B7.1 
wiggler: 30 poles: $B=13.0 \mathrm{hG}: \mathrm{lambda}=12.85 \mathrm{~cm}$

$0.5 \mathrm{~m}$ from center of wiggler to mirror

electron energy $=3.40$ e+00 GeV critical energy $=1.00 \mathrm{e}+01 \mathrm{heV}$
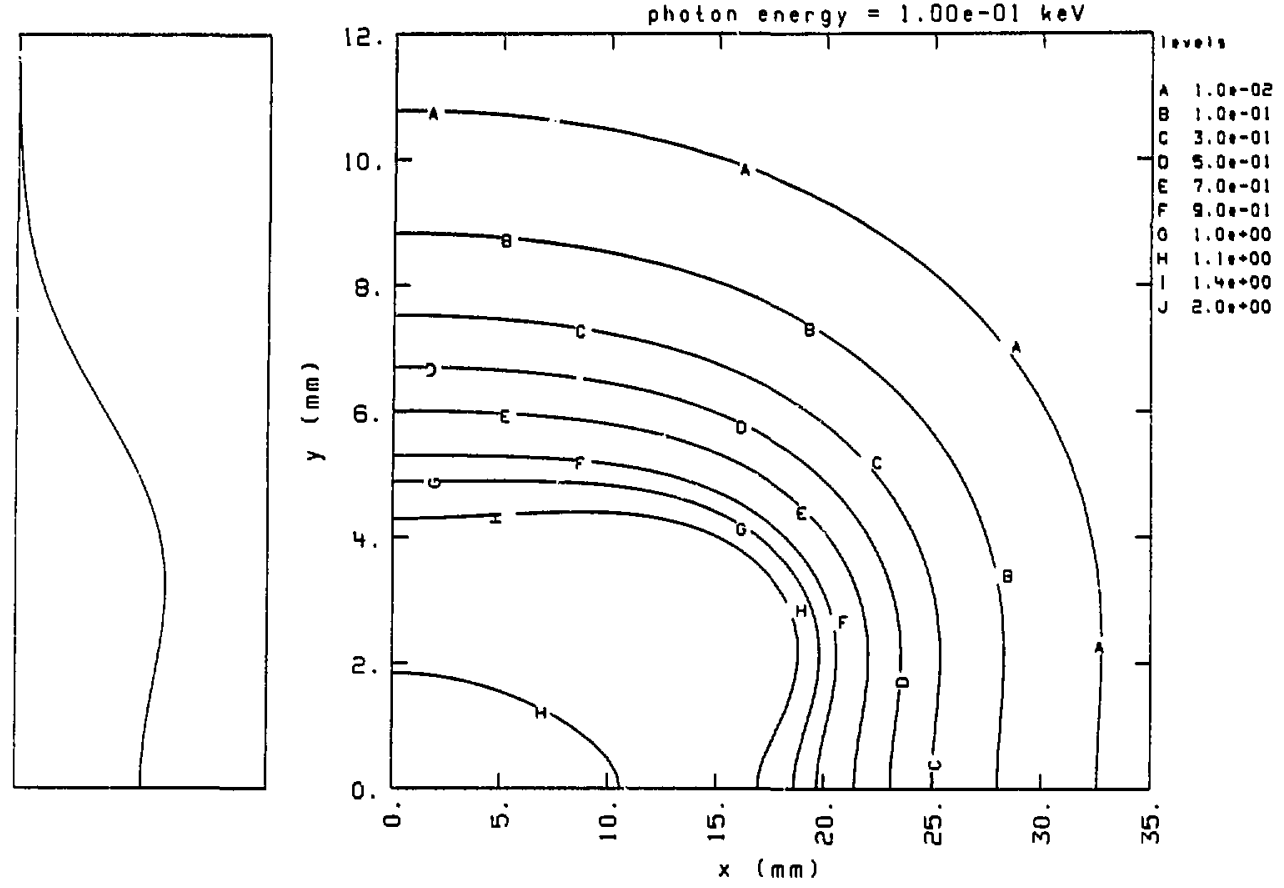

$$
\begin{aligned}
\text { normalized to } \\
\begin{aligned}
z & =8.78 \mathrm{e}+09 \\
\text { at } x & =0 . \\
y & =0 .
\end{aligned}
\end{aligned}
$$

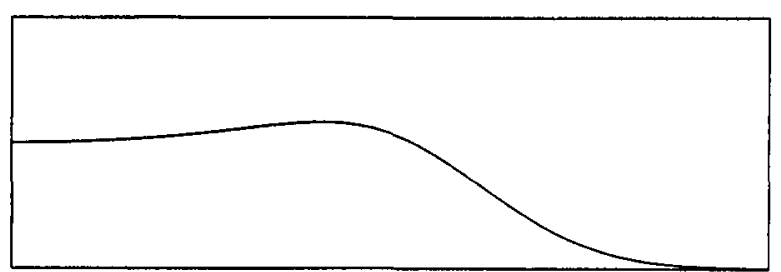

Fig. $B 7.2$ 


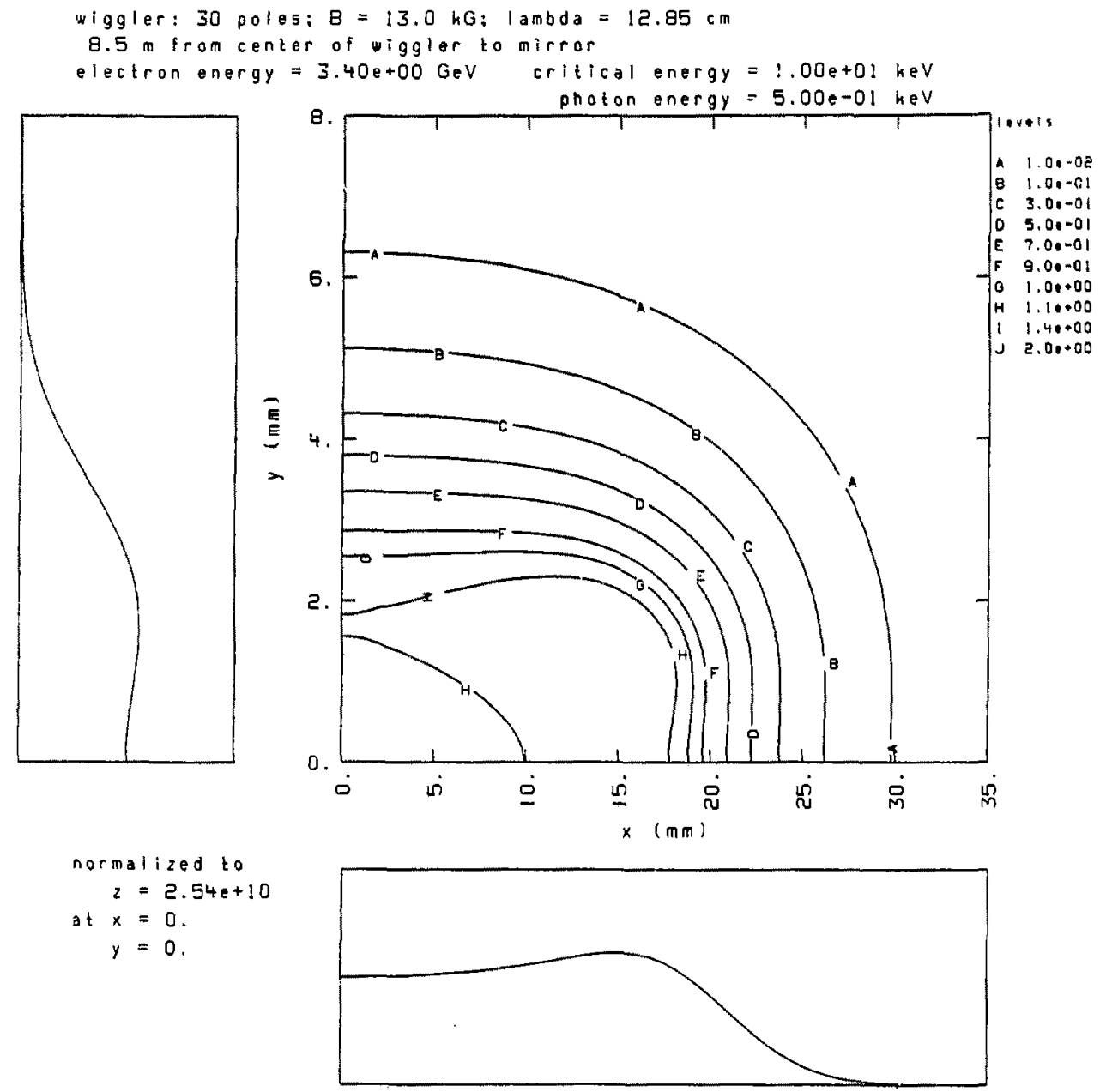

Fig. B7.3. 

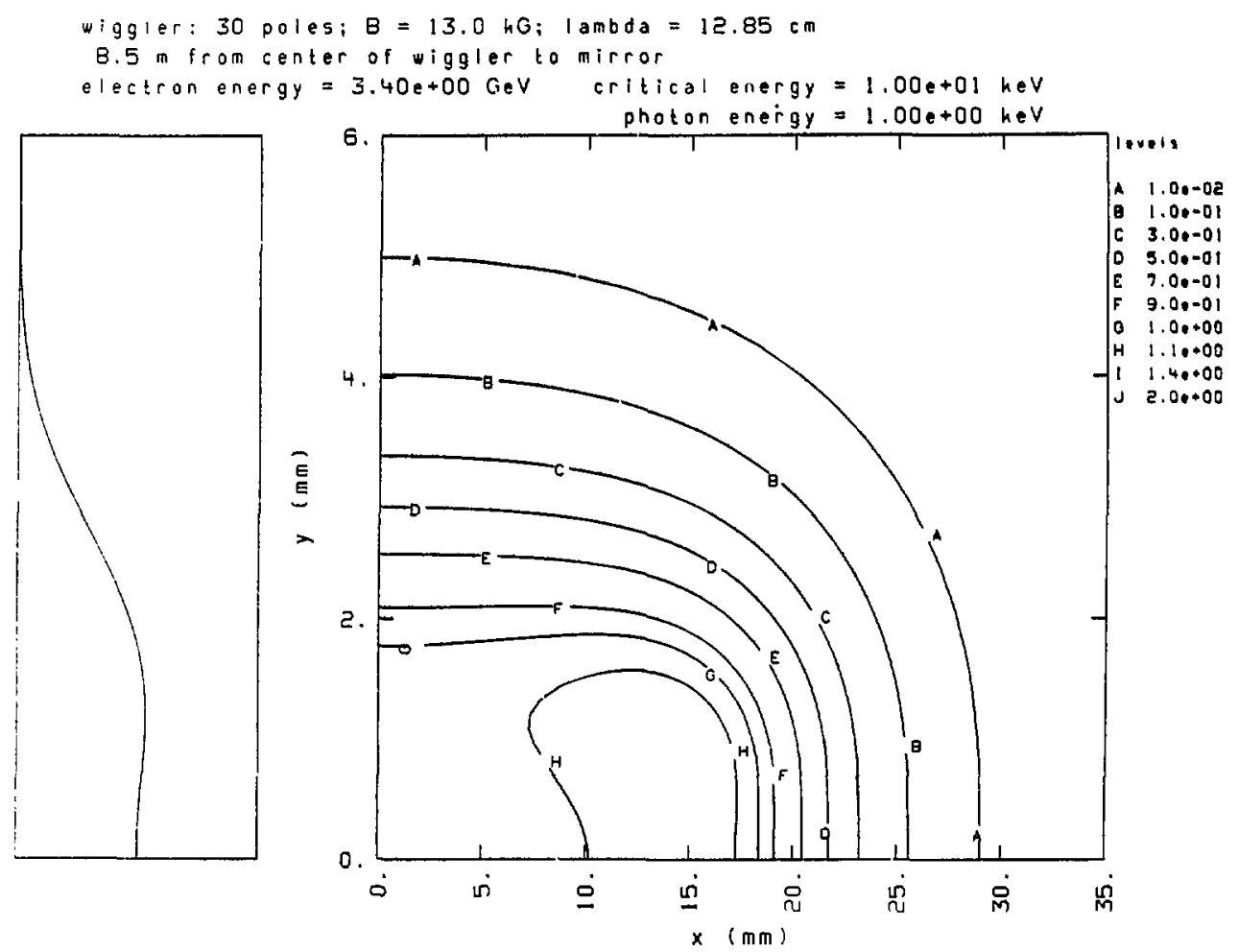

narmalized to

$z=3.95 \mathrm{e}+10$

at $x=0$.

$y=0$.

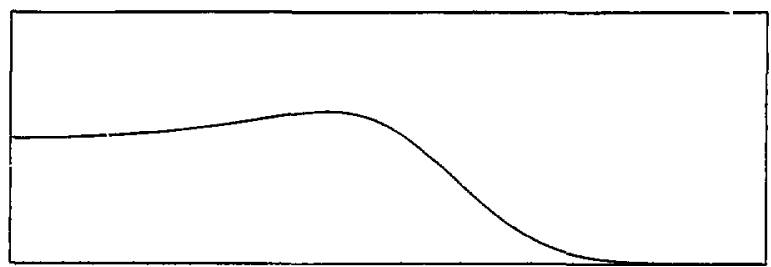

Fig. B7.4. 


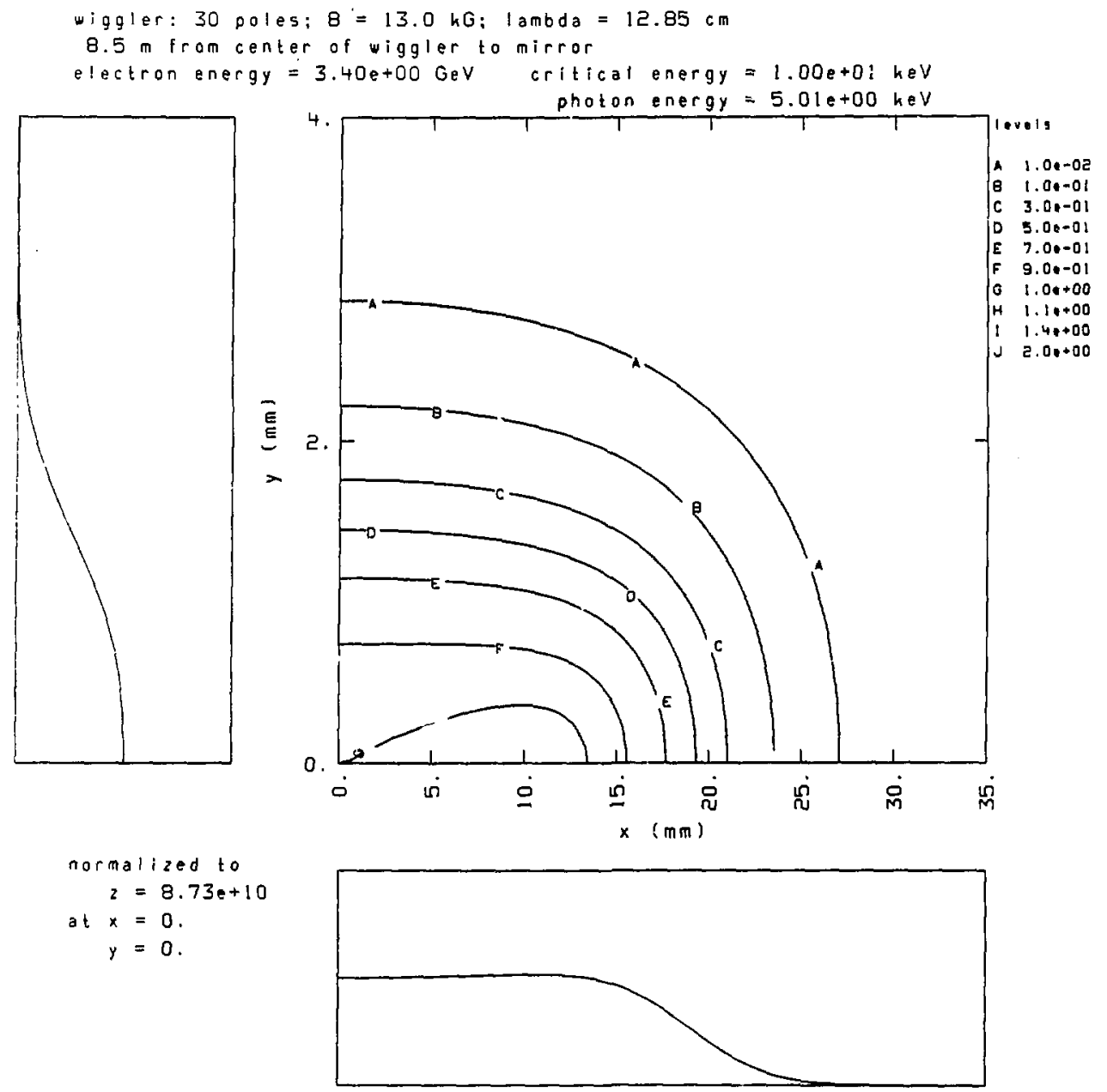

Fig. 87.5 . 


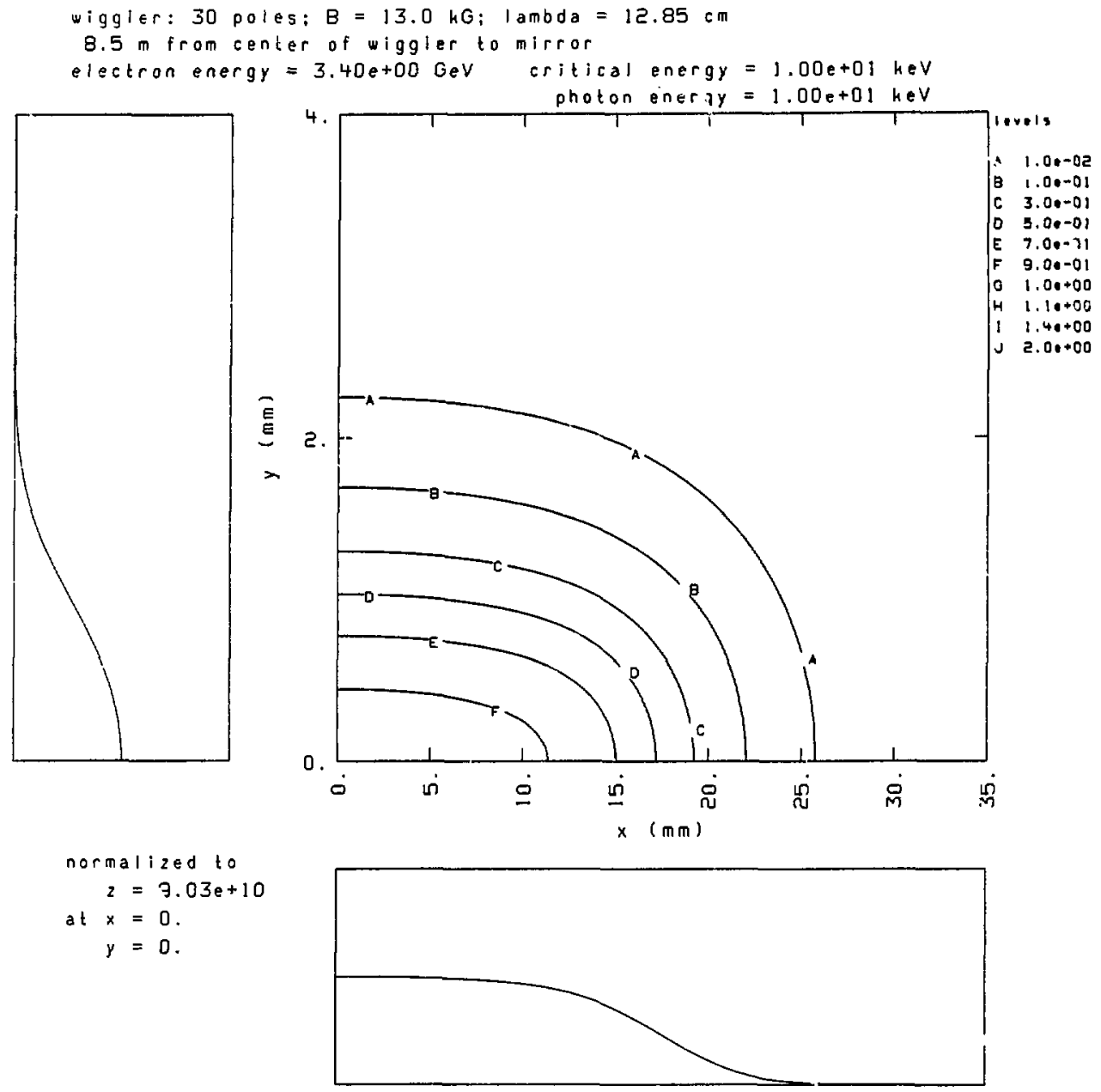

Fig. B7.6. 


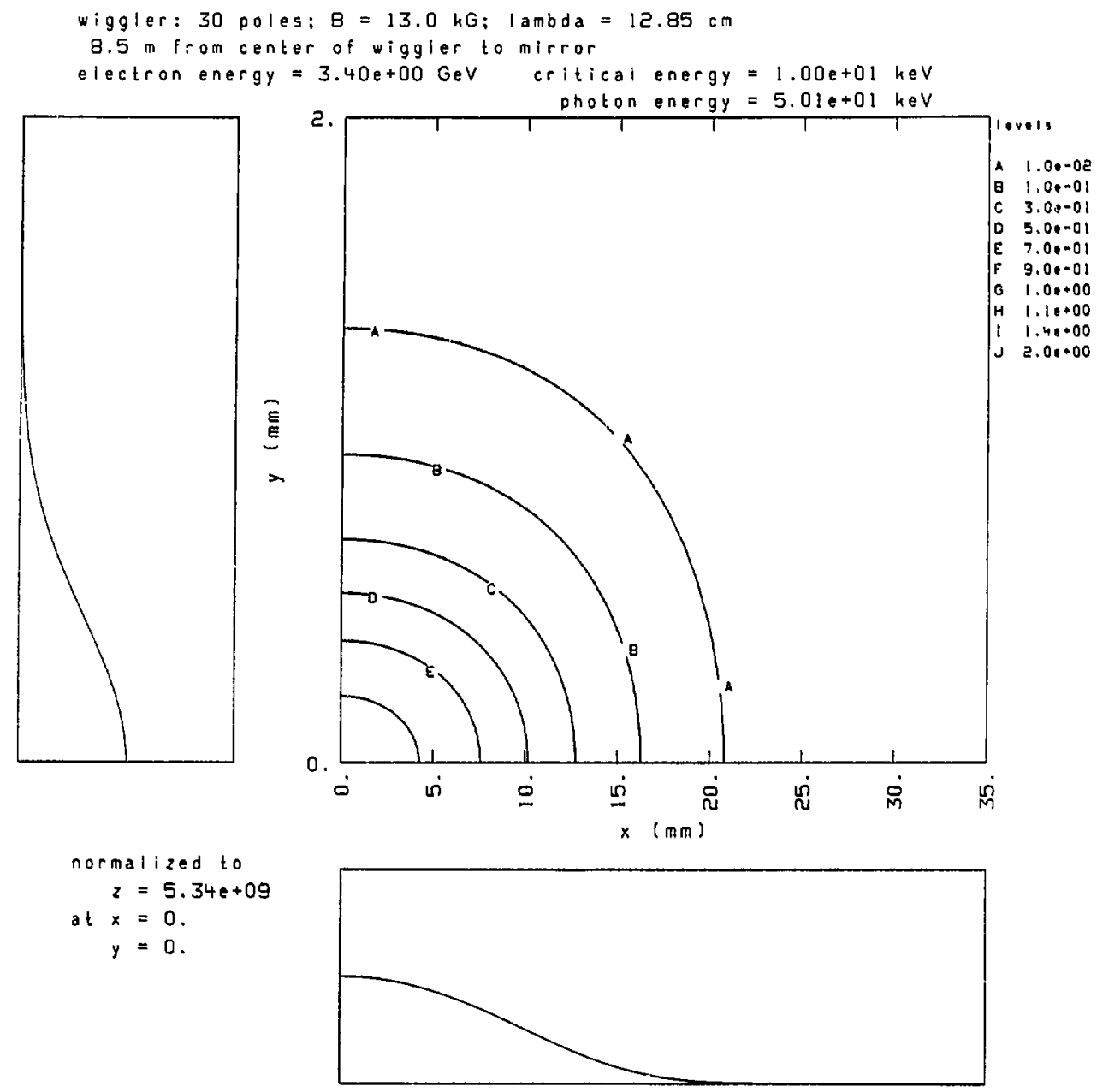

Fig. B7.7. 
wiggler: 30 poles: $B=13.0 \mathrm{kG}: 1 \mathrm{ambda}=12.85 \mathrm{~cm}$

$8.5 \mathrm{~m}$ from center of wiggler to mirror

electron energy $=3.40 \mathrm{e}+00 \mathrm{GeV}$ crikical enorgy $=1.00 \mathrm{e}+01 \mathrm{keV}$
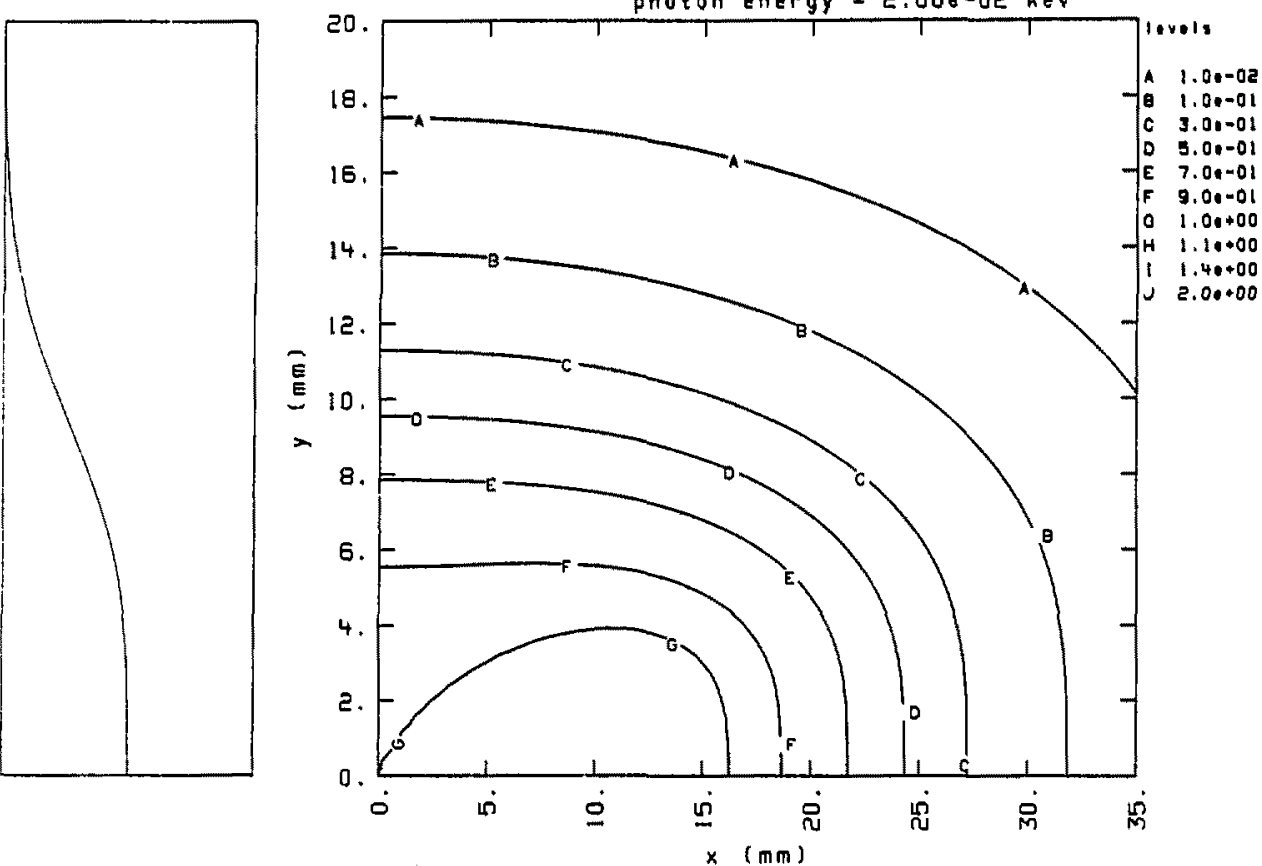

paralle!

polarization

normalized to

$z=2.95 e+09$

at $x=0$.

$\ddot{y}=0$.

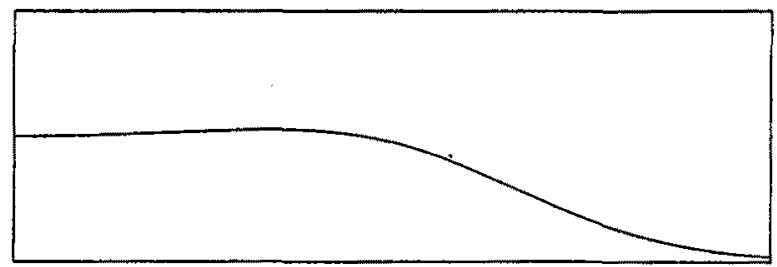

Fig. B8.1. 


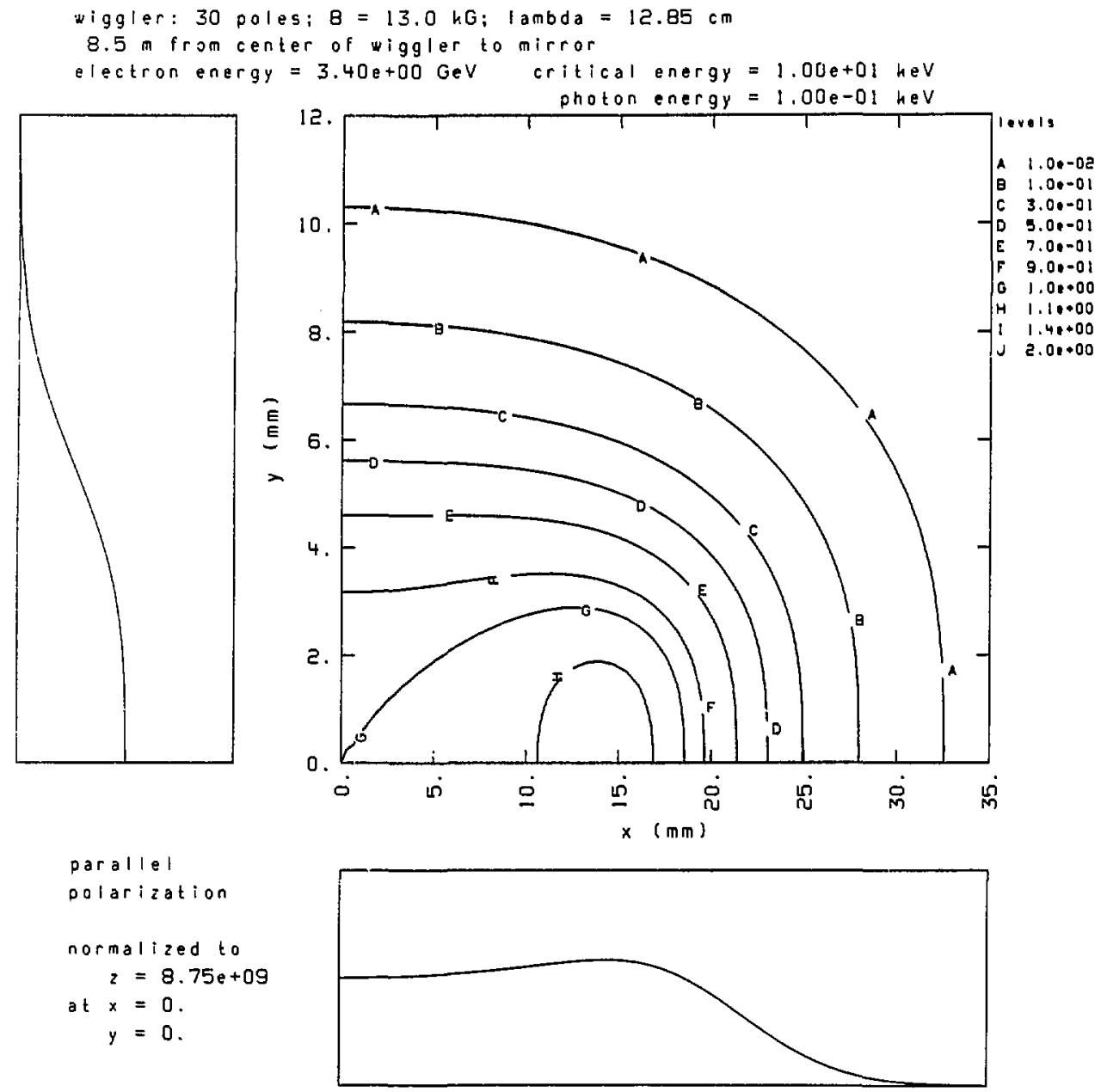

Fig. B8.2. 


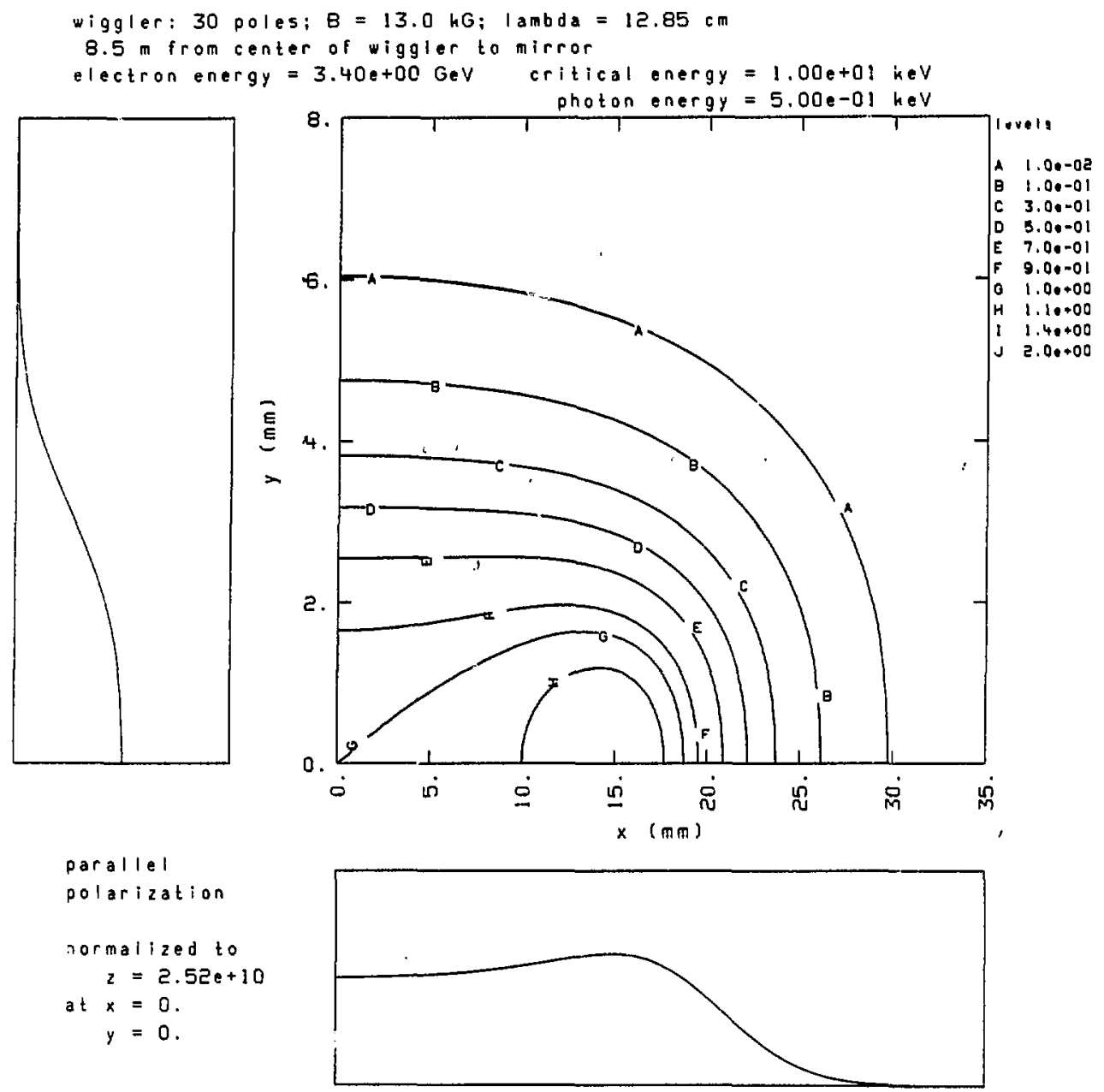

Fig. B8.3. 


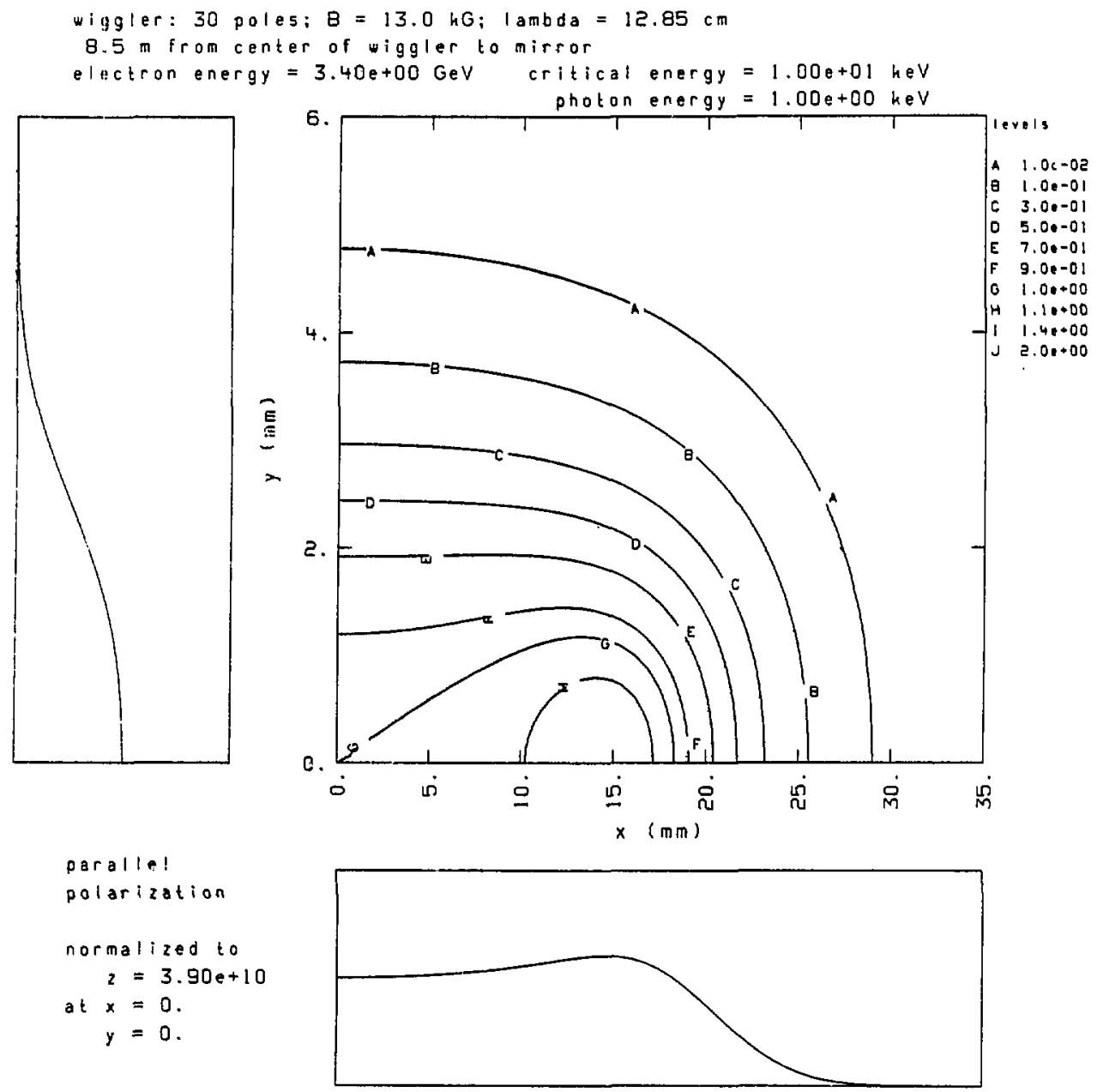

Fig. B8.4. 
wiggler: 30 poles; $B=13.0 \mathrm{kG}$; lambda $=12.85 \mathrm{~cm}$

$8.5 \mathrm{~m}$ from center of wigler to mirror

electron energy $=3.40 \mathrm{e}+00 \mathrm{GeV}$ critical energy $=1.000+01 \mathrm{heV}$

photon energy $=5.010+00 \mathrm{hoV}$
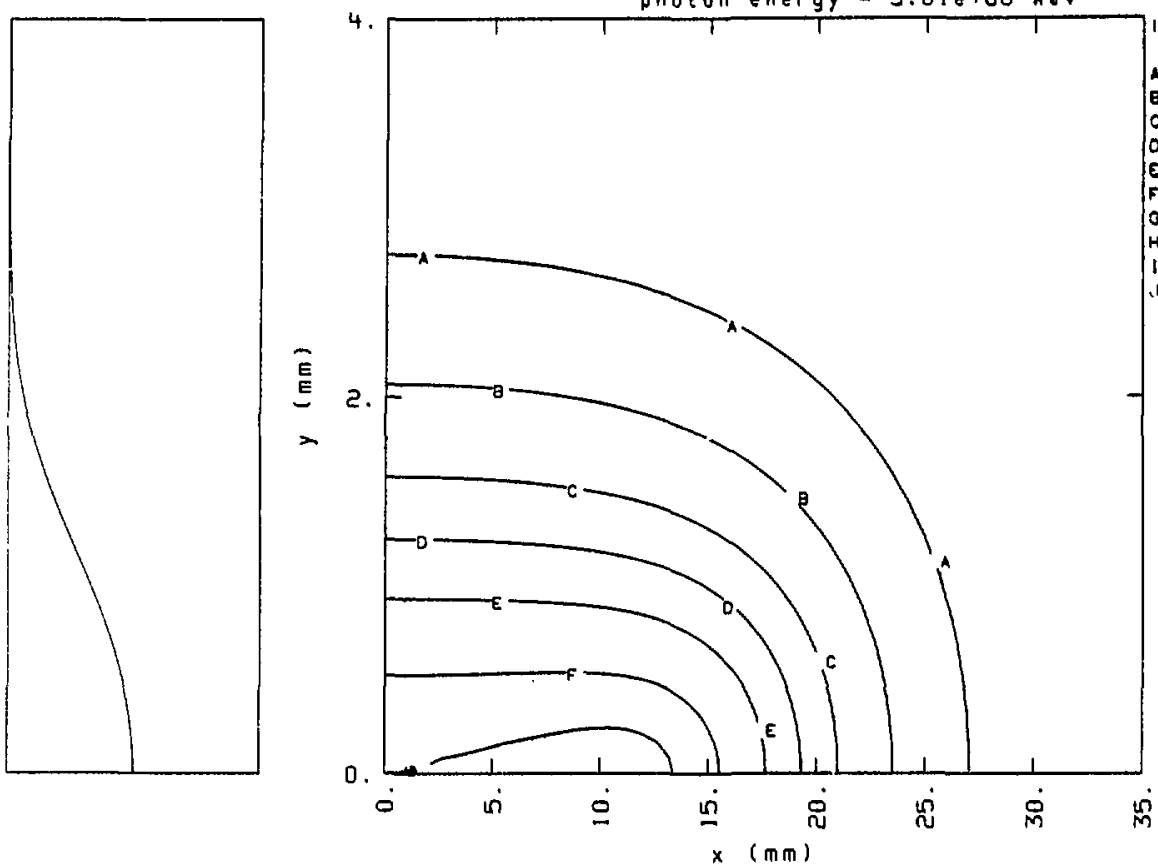

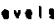

1.0 .02

a $\quad 1.0,-01$

C 3.0 .0 .01

0 5.0.-01

E 7.00 .01

F $9.01-01$

o $1.00+00$

H $1,10+00$

$1.4,+00$

$2.00+00$

$$
\begin{aligned}
& \text { parallel } \\
& \text { polarization } \\
& \text { normalized to } \\
& \qquad \begin{aligned}
z & =8.51 e+10 \\
\text { at } x & =0 . \\
y & =0 .
\end{aligned}
\end{aligned}
$$

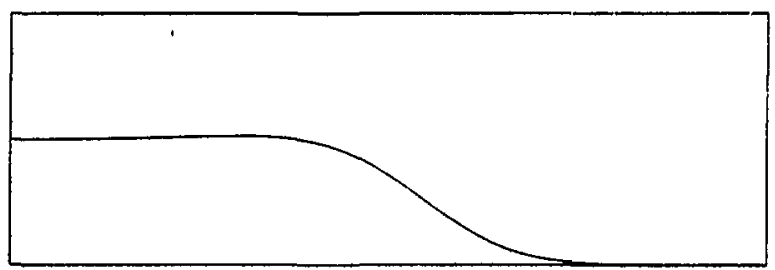

Fig. B8.5. 


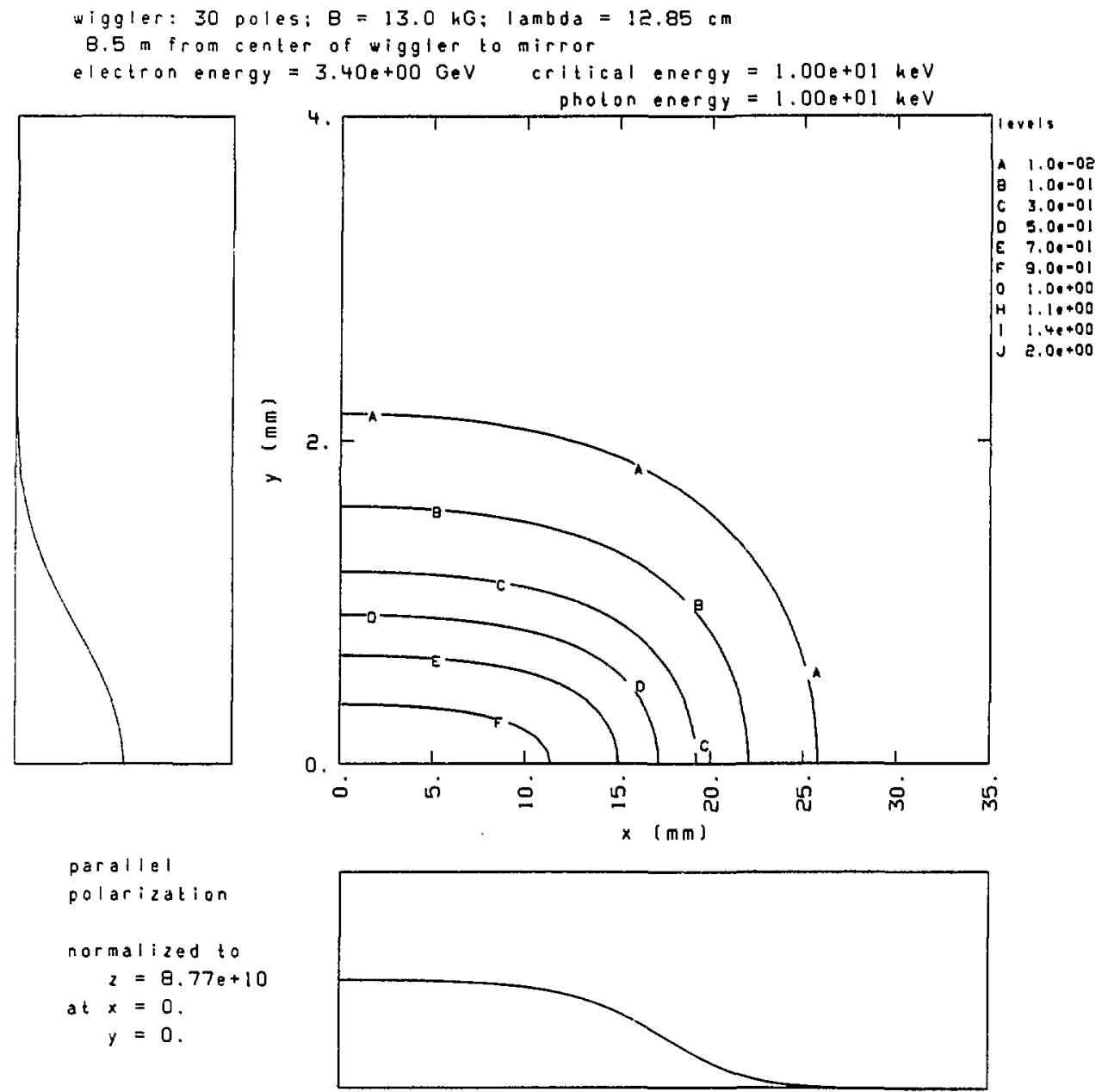

Fig. B8.6. 


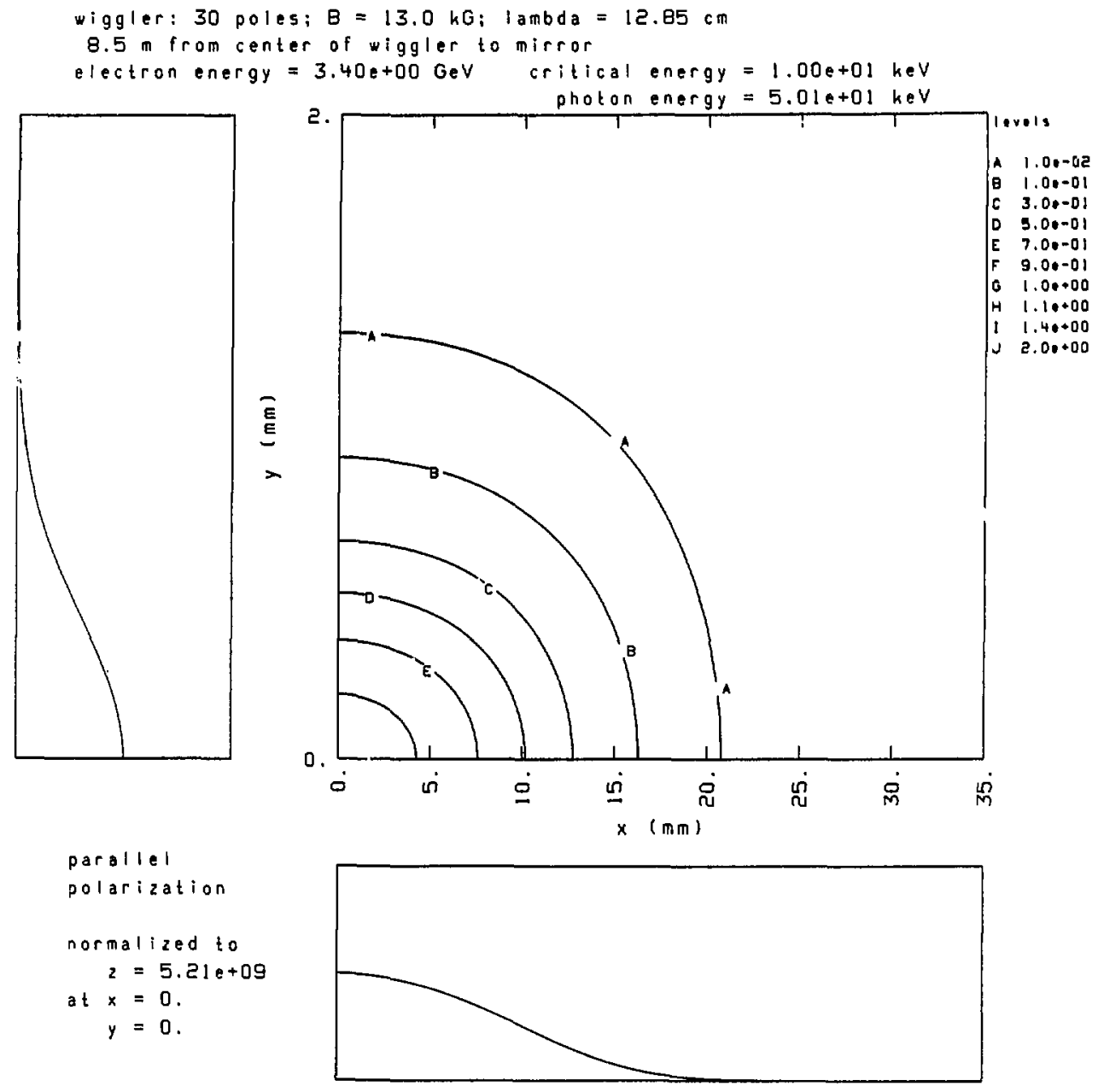

Fig. B8.7. 

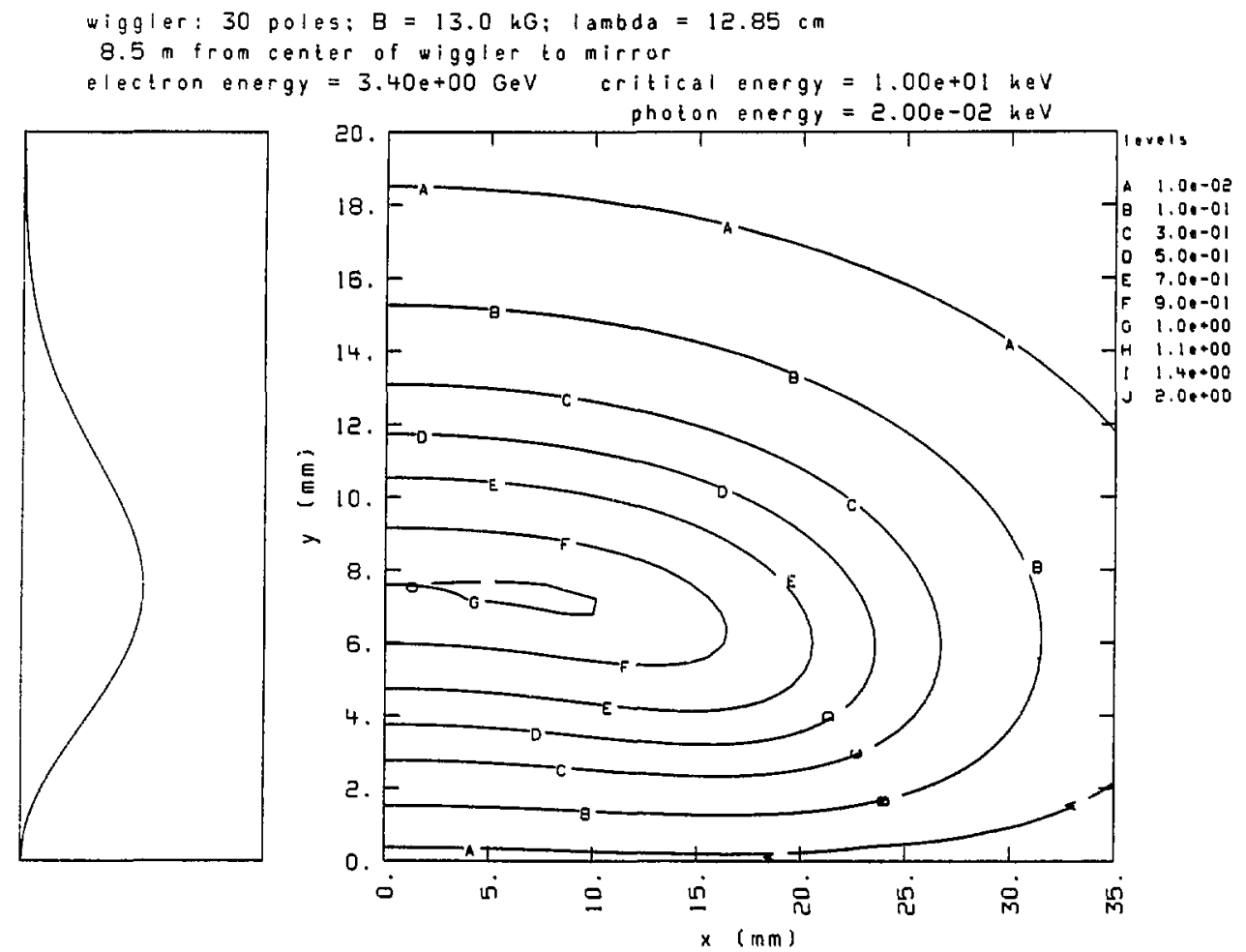

perpendicular polarization

normalized to

$z=1.13 e+09$

a $x=0$.

$y=7.60 e+00$

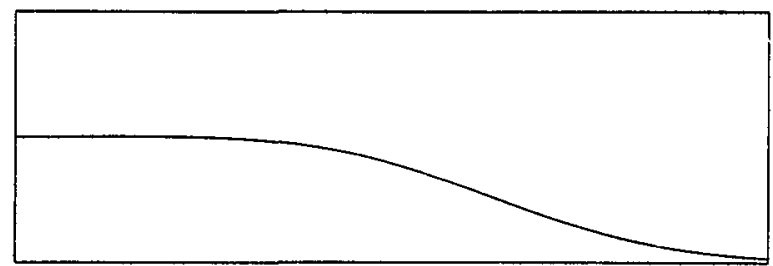

Fig. B9.1. 

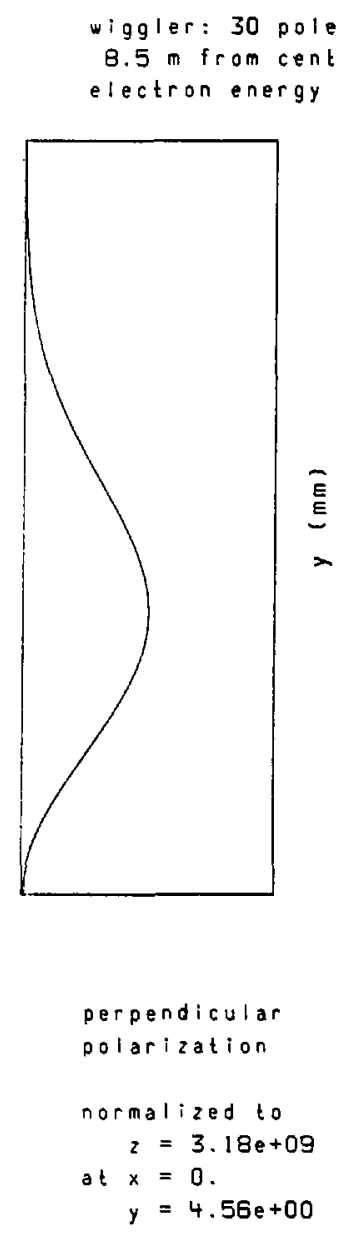

$$
\begin{aligned}
& \text { oles: } B=13.0 \mathrm{kG} \text {; lambda }=12.85 \mathrm{~cm} \\
& \text { center of wiggler to mirror }
\end{aligned}
$$

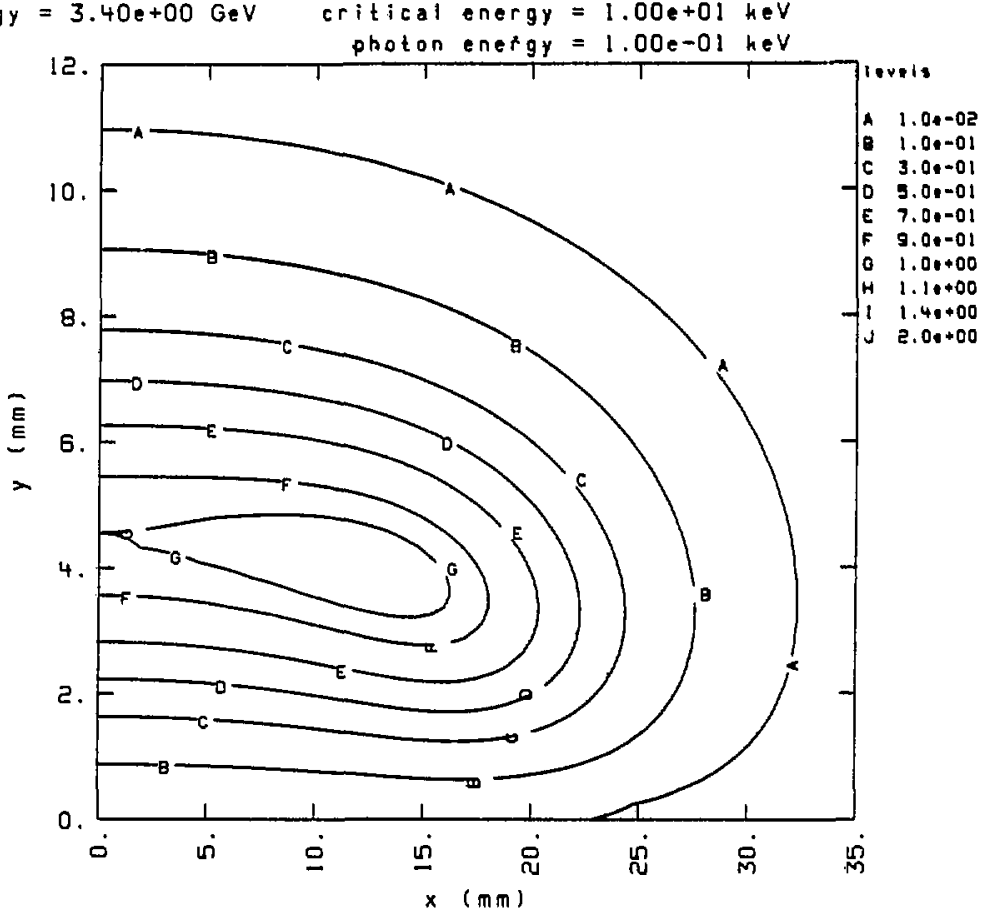

perpendicular

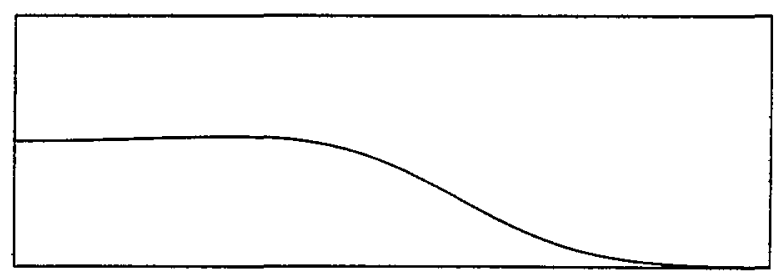

Fig. B9.2. 

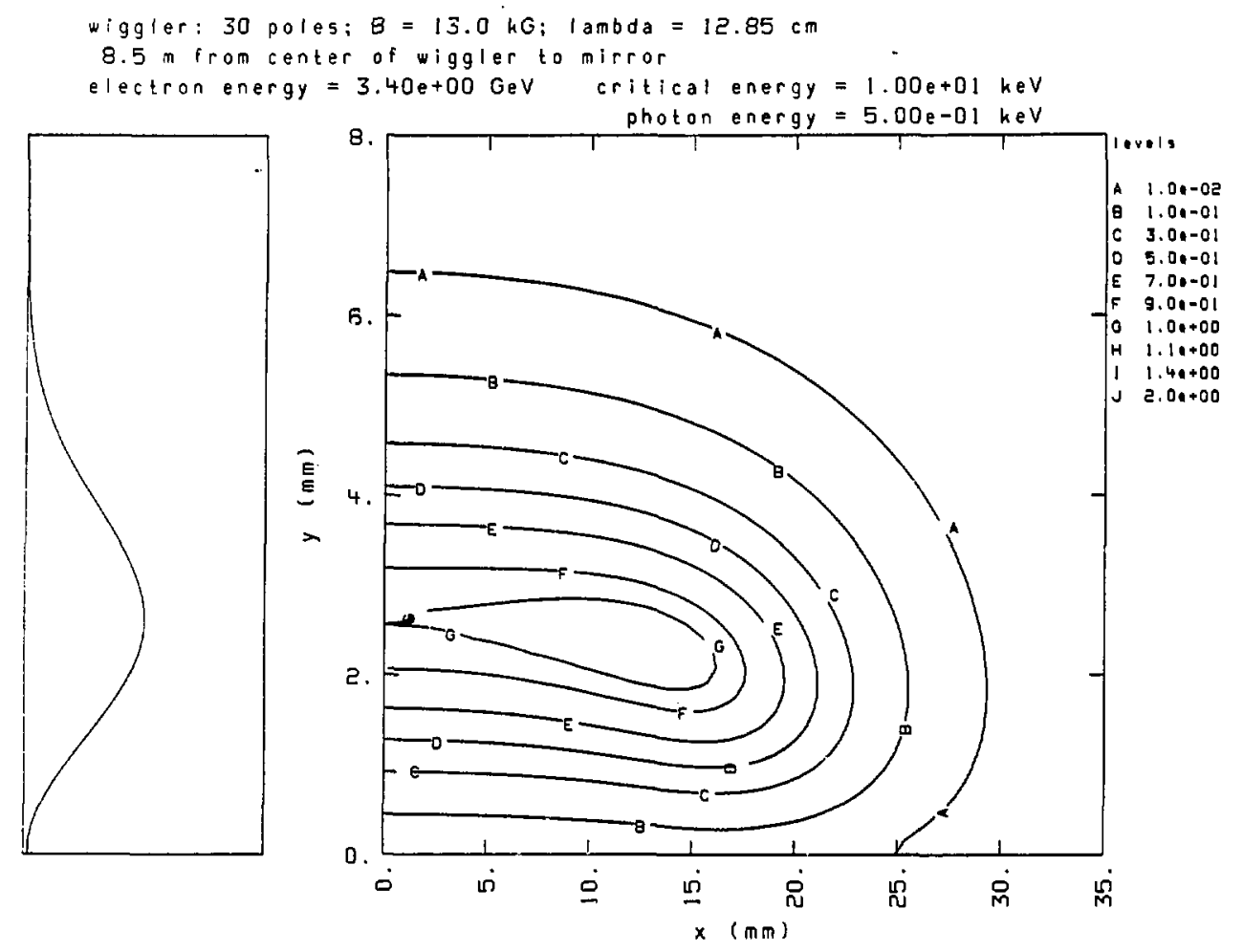

perpendicular polarization

normalized to

$z=7.88 \mathrm{e}+09$

at $x=0$.

$y=2.56 \mathrm{e}+00$

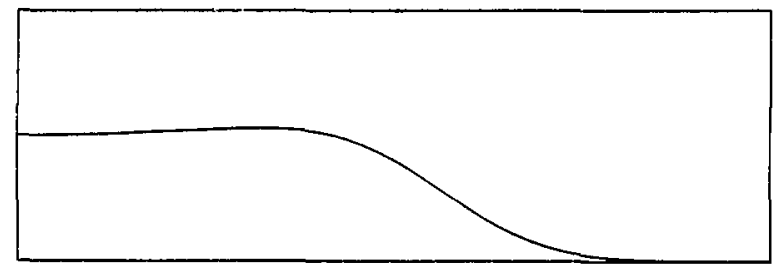

Fig. 89.3. 
wiggler: 30 poles: $B=13.0 \mathrm{kG}$ : lambda $=12.85 \mathrm{~cm}$

$8.5 \mathrm{~m}$ from center of iggler to mirror

electron energy $=3.40 \mathrm{e}+00 \mathrm{GeV}$ critical energy $=1.00 \mathrm{e}+01 \mathrm{heV}$
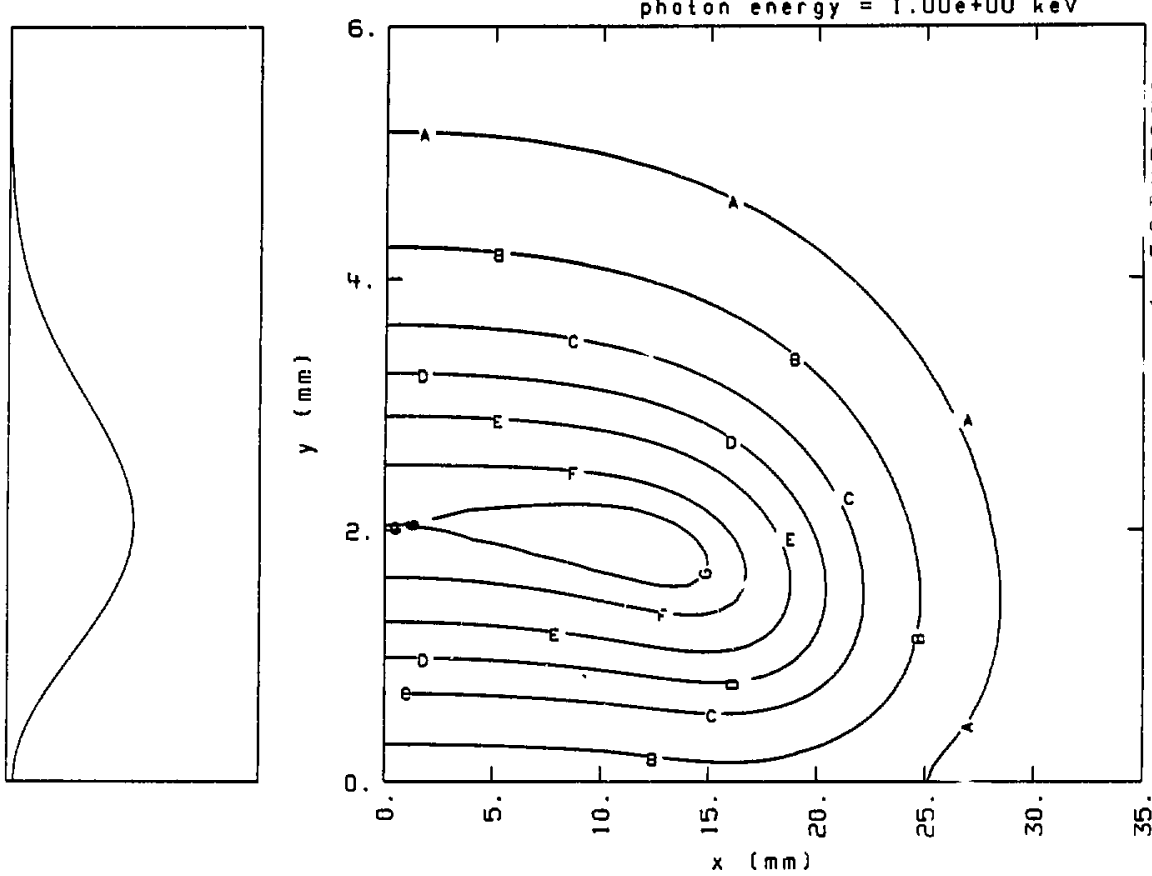

Itvels

A $\quad$. Opr-oe

$3 \quad 1.06-01$

c $3.0 \cdot-01$

$0.0,-01$

$=7.0 \cdot-01$

9.0.-01

$1.0 ?+00$

H $1.1,10+00$

$-1 \quad 1,4 e+00$

J $2.00+0 C$

perpendicular polarization

normalized to

$z=1.07 \mathrm{e}+10$

at $x=0$.

$y=2.04 e+00$

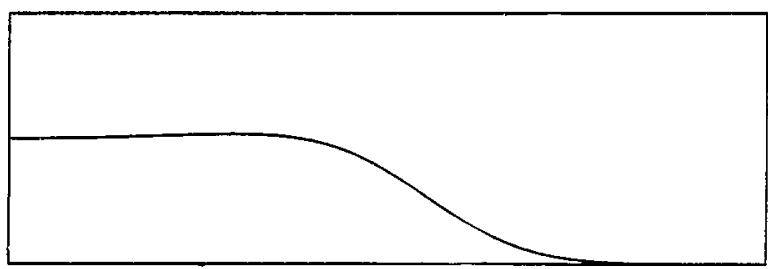

Fig. B9.4. 
wigler: 30 poles: $B=13.0 \mathrm{hG} ; 1$ ambda $=12.85 \mathrm{~cm}$

$8.5 \pi$ from center of wiggler to mirror

electron energy $=3.40 \mathrm{e}+00 \mathrm{GeV}$ criticat energy $=1.00 \mathrm{e}+0 \mathrm{l} \mathrm{heV}$
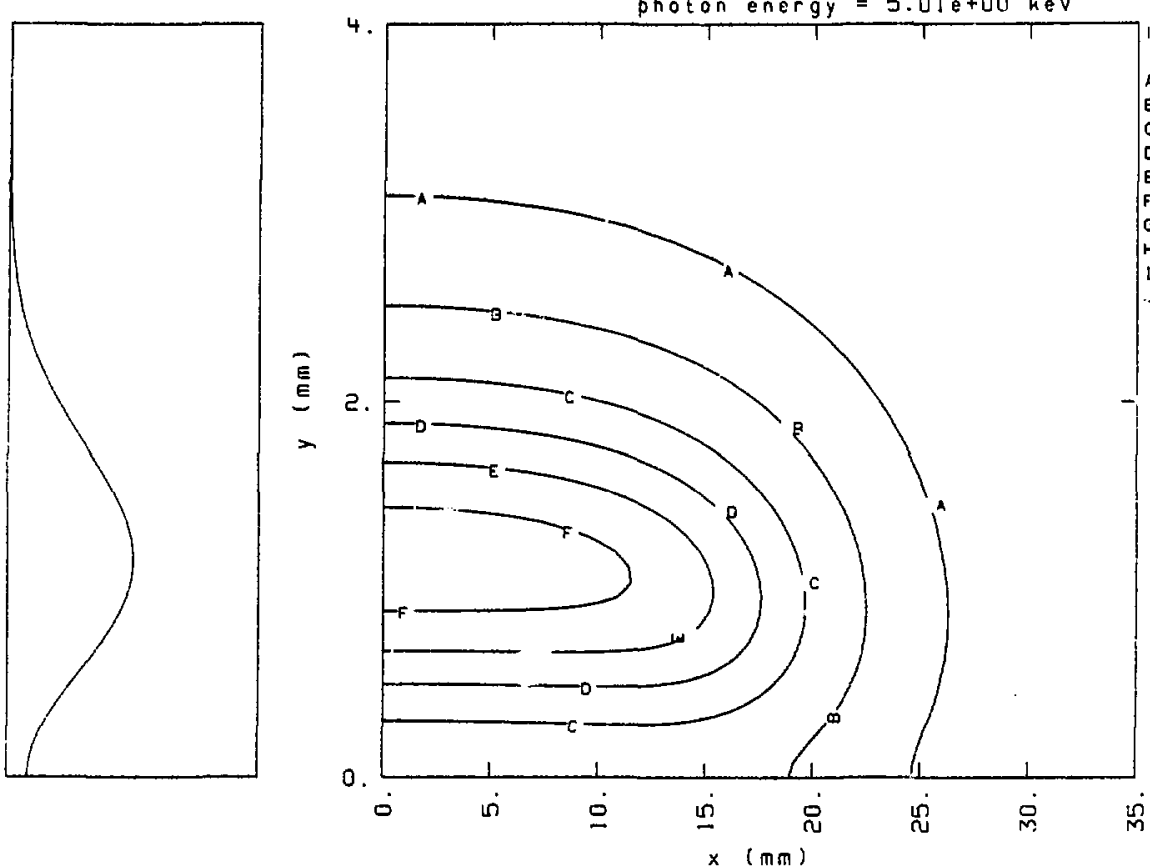

perpendicular polarization

normalized to

$z=1.35 \mathrm{e}+10$

at $x=0$.

$y=1.12 t+00$

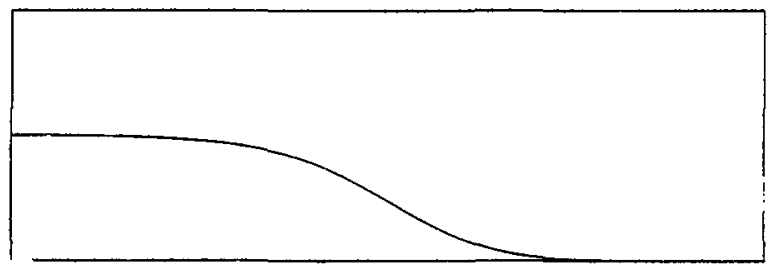

Fig. B9.5. 
wiggler: 30 poles: $8=13.0 \mathrm{kG}: 1$ ambda $=12.85 \mathrm{~cm}$

$8.5 \mathrm{~m}$ from center of wiggler to mirror

electron energy $=3.40 \mathrm{e}+00 \mathrm{GeV}$ critical energy $=1.00 \mathrm{e}+01 \mathrm{keV}$
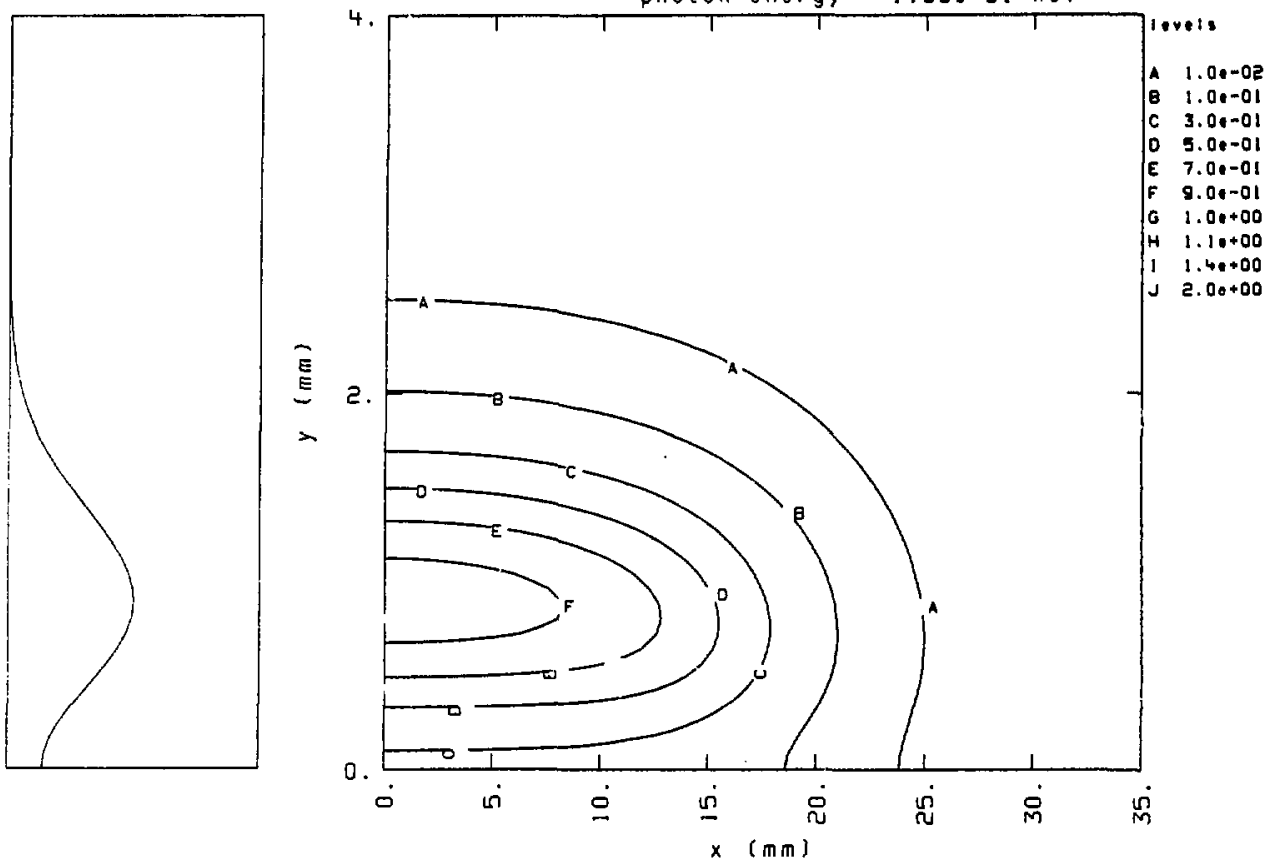

perpendicular polarization

normalized to

$z=9.42 e+09$

at $x=0$.

$y=8.80 c-01$

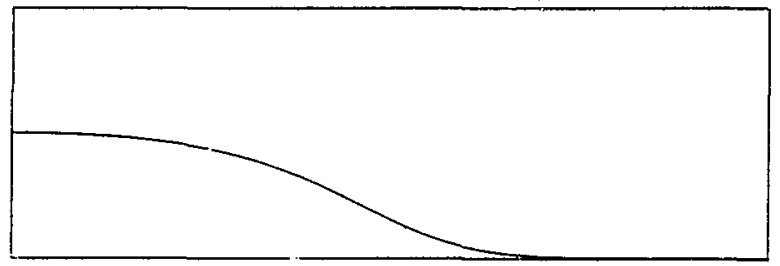

Fig. 89.6. 
wiggler: 30 poles; $B=13.0 \mathrm{kG} ; 1$ ambda $=12.85 \mathrm{~cm}$

8.5 m from center of wiggler to mirror

electron energy $=3.40$ eto GeV critical energy $=1.00 \mathrm{e}+01 \mathrm{keV}$
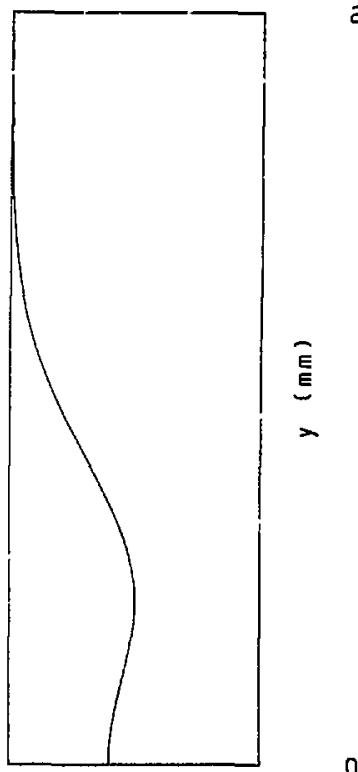

2.

photon energy $=5.01+0$ l keV

0 .

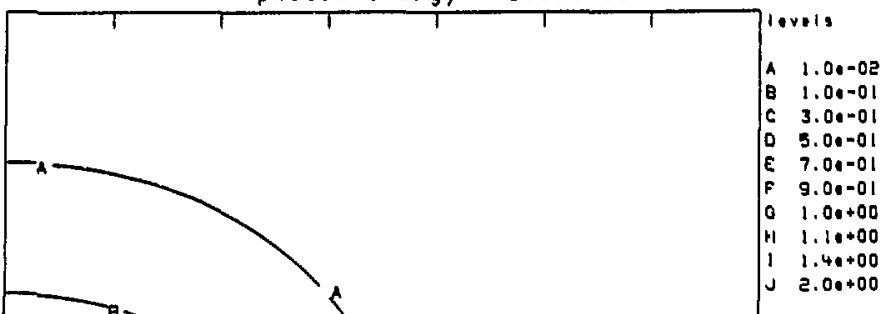

perpendicular polarization

normalized to

$z=1.63 e+08$

at $x=0$.

$y=4.40 e-01$

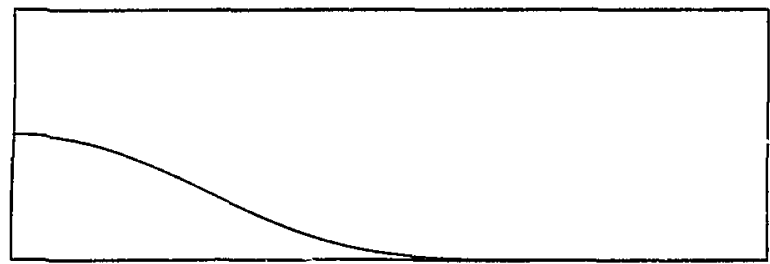

Fig. : B9.7. 


\section{APPENDIX C}

TABLES OF INTEGRATED ABSOLUTE NUMBERS OF PHOTONS

List of tables:

Total intensity for $E=1.8 \mathrm{GeV}\left(\varepsilon_{c}=2.80 \mathrm{keV}\right)$

\begin{tabular}{lr} 
Table & Photon Energy \\
\hline C1.1 & $20 \mathrm{eV}$ \\
C1.2 & $100 \mathrm{eV}$ \\
C1.3 & $500 \mathrm{eV}$ \\
C1.4 & $1 \mathrm{keV}$ \\
C1.5 & $E_{\mathrm{c}} / 2$ \\
C1.6 & 50 \\
C1.7 & $5 \mathrm{E}_{\mathrm{s}}$
\end{tabular}

Parallel polariestion intensity for $E=1.8 \mathrm{GeV}\left(\epsilon_{\mathrm{c}}=2.80 \mathrm{keV}\right)$

\begin{tabular}{lr} 
Table & Photon Energy \\
\hline C2.1 & $20 \mathrm{eV}$ \\
$\mathrm{C} 2.2$ & $100 \mathrm{eV}$ \\
$\mathrm{C} 2.3$ & $500 \mathrm{eV}$ \\
$\mathrm{C2} .4$ & $1 \mathrm{keV}$ \\
$\mathrm{C} 2.5$ & $\varepsilon_{\mathrm{c}} / 2$ \\
$\mathrm{C2} .6$ & $\varepsilon_{\mathrm{c}}$ \\
$\mathrm{C} 2.7$ & $5 \varepsilon_{\mathrm{c}}$
\end{tabular}

Perpendicular polarization intensity for $E=1.8 \mathrm{GeV}\left(\varepsilon_{c}=2.80 \mathrm{keV}\right)$

\begin{tabular}{lr} 
Table & Photon Energy \\
\hline C3.1 & $20 \mathrm{eV}$ \\
C3.2 & $100 \mathrm{eV}$ \\
C3.3 & $500 \mathrm{eV}$ \\
C3.4 & $1 \mathrm{keV}$ \\
C3.5 & $\varepsilon_{\mathrm{c}} / 2$ \\
C3.6 & $\varepsilon_{c}$ \\
C3.7 & $5 \varepsilon_{c}$
\end{tabular}

Total intensity for $E=3.0 \mathrm{GeV}\left({ }_{-c}=7.78 \mathrm{keV}\right)$

\begin{tabular}{lr} 
Table & Zioton Energy \\
\hline$C 4.1$ & $20 \mathrm{eV}$ \\
$C 4.2$ & $100 \mathrm{eV}$ \\
$\mathrm{C} 4.3$ & $500 \mathrm{eV}$ \\
$\mathrm{C} 4.4$ & $1 \mathrm{keV}$ \\
$\mathrm{C} 4.5$ & $\varepsilon_{\mathrm{c}} / 2$ \\
$\mathrm{C} 4.6$ & $\varepsilon_{\mathrm{c}}$ \\
$\mathrm{C} 4.7$ & $5 \mathrm{E}_{\mathrm{c}}$
\end{tabular}


Parallel polarization intensity for $E=3.0 \mathrm{GeV}\left(\varepsilon_{c}=7.78 \mathrm{keV}\right)$

\begin{tabular}{lr} 
Table & Photon Energy \\
\hline C5.1 & $20 \mathrm{eV}$ \\
C5.2 & $100 \mathrm{eV}$ \\
C5.3 & $500 \mathrm{eV}$ \\
$C 5.4$ & $1 \mathrm{keV}$ \\
$C 5.5$ & $\varepsilon_{\mathrm{c}} / 2$ \\
$C 5.6$ & $\varepsilon_{\mathrm{c}}$ \\
$\mathrm{C5} .7$ & $5 \varepsilon_{\mathrm{c}}$
\end{tabular}

Perpendicular polarization intensity for $E=3.0 \mathrm{GeV}\left(\varepsilon_{c}=7.78 \mathrm{keV}\right)$

\begin{tabular}{lr} 
Table & Photon Energy \\
\hline C6.1 & $20 \mathrm{eV}$ \\
$\mathrm{C} 6.2$ & $100 \mathrm{eV}$ \\
$\mathrm{C} 6.3$ & $500 \mathrm{eV}$ \\
$\mathrm{C} 6.4$ & $1 \mathrm{keV}$ \\
$\mathrm{C} 6.5$ & $\varepsilon_{\mathrm{c}} / 2$ \\
$\mathrm{C} 6.6$ & $\varepsilon_{\mathrm{c}}$ \\
$\mathrm{C} 6.7$ & $5 \varepsilon_{\mathrm{c}}$
\end{tabular}

Totel intensity for $E=3.4 \mathrm{GeV}\left(\varepsilon_{c}=10.00 \mathrm{keV}\right)$

\begin{tabular}{lr} 
Table & Photon Energy \\
\hline C7.1 & $20 \mathrm{eV}$ \\
$c 7.2$ & $100 \mathrm{eV}$ \\
$c 7.3$ & $500 \mathrm{eV}$ \\
$c 7.4$ & $1 \mathrm{keV}$ \\
$c 7.5$ & $\varepsilon_{\mathrm{c}} / 2$ \\
$c 7.6$ & $\varepsilon_{\mathrm{c}}$ \\
$\mathrm{c7} .7$ & $5 \varepsilon_{\mathrm{c}}$
\end{tabular}

Parallel polarization intensity for $E=3.4 \mathrm{GeV}\left(\varepsilon_{\mathrm{c}}=10.00 \mathrm{keV}\right)$

\begin{tabular}{lr} 
Table & Photon Energy \\
\hline C8.1 & $20 \mathrm{eV}$ \\
$\mathrm{C8} .2$ & $100 \mathrm{eV}$ \\
$\mathrm{C8} .3$ & $500 \mathrm{eV}$ \\
$\mathrm{C8.4}$ & $1 \mathrm{keV}$ \\
$\mathrm{C8.5}$ & $\varepsilon_{\mathrm{c}} / 2$ \\
$\mathrm{C8.6}$ & $\varepsilon_{\mathrm{c}}$ \\
$\mathrm{C8.7}$ & $5 \varepsilon_{\mathrm{c}}$
\end{tabular}

Perpendicular polarization intensity for $\mathrm{E}=3.4 \mathrm{GeV}\left(\varepsilon_{\mathrm{c}}=10.00 \mathrm{keV}\right)$

\begin{tabular}{lr} 
Table & Photon Energy \\
\hline$C 9.1$ & $20 \mathrm{eV}$ \\
$\mathrm{C} .2$ & $100 \mathrm{eV}$ \\
$\mathrm{C9.3}$ & $500 \mathrm{eV}$ \\
$\mathrm{C} .4 .4$ & $1 \mathrm{keV}$ \\
69.5 & $\varepsilon_{\mathrm{c}} / 2$ \\
$\mathrm{C} .6$ & $\varepsilon_{\mathrm{c}}$ \\
$\mathrm{C} 9.7$ & $5 \varepsilon_{\mathrm{c}}$
\end{tabular}


Table C1.1.

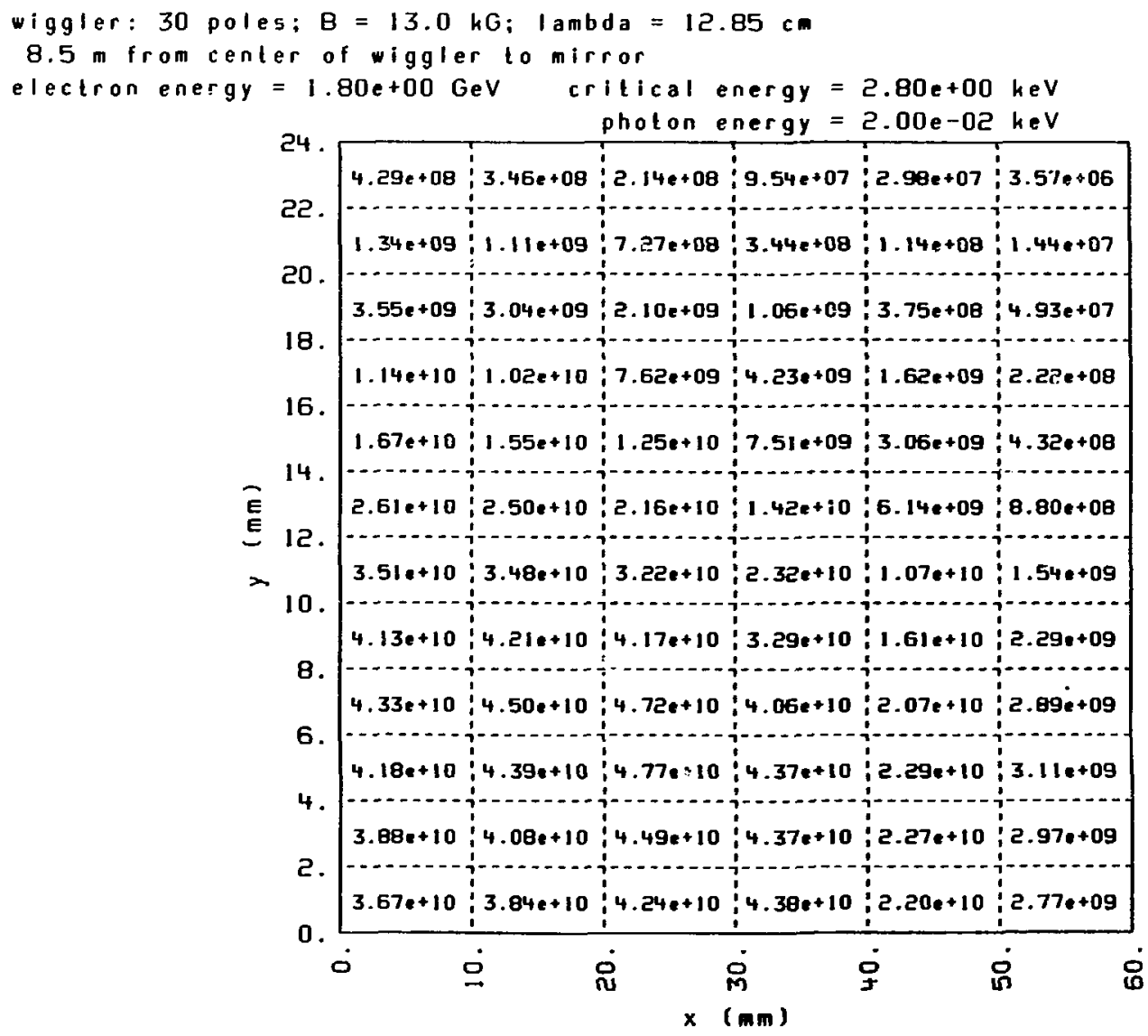

photons/sec/mA in 0.17 bandwidth 


\section{Table Cl.2.}

wiggler: 30 poles; $B=13.0 \mathrm{hG} ; 1 \mathrm{ambda}=12.85 \mathrm{~cm}$

$8.5 \mathrm{~m}$ from center of wiggler to mirror

electron energy $=1.80 \mathrm{e}+00 \mathrm{GeV}$ crilical energy $=2.80 \mathrm{e}+00 \mathrm{heV}$ photon energy $=1.00 \mathrm{e}-01 \mathrm{heV}$

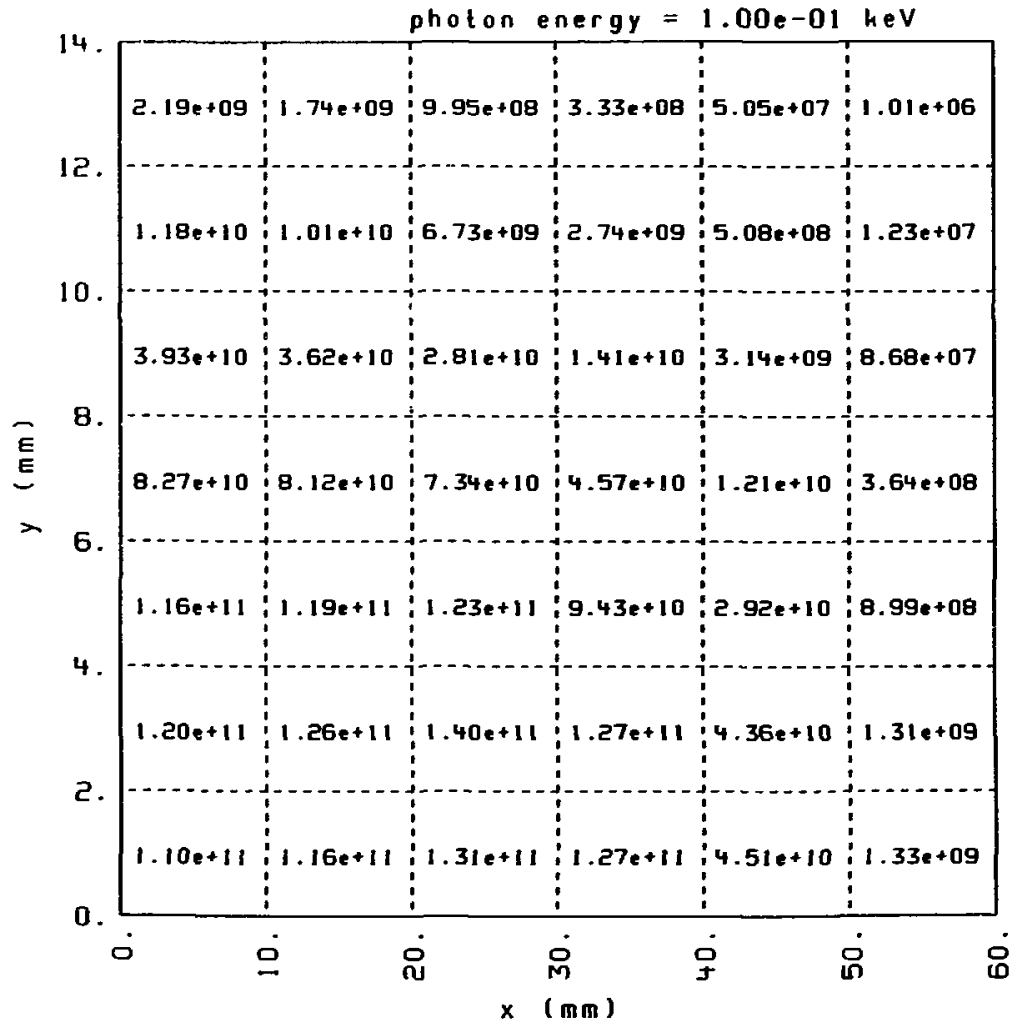

photons/sec/mA in $0.1 \%$ bandwidth 
Table Ci.3.

wiggler: 30 poles: $B=13.0 \mathrm{hG} ; 1$ ambda $=12.85$

$8.5 \mathrm{~m}$ from center of wiggler to mirror

electron energy $=1.80 \mathrm{e}+00 \mathrm{GeV}$ critical energy $=2.80 \mathrm{e}+00 \mathrm{heV}$

photon energy $=5.00 \mathrm{e}-01 \mathrm{keV}$

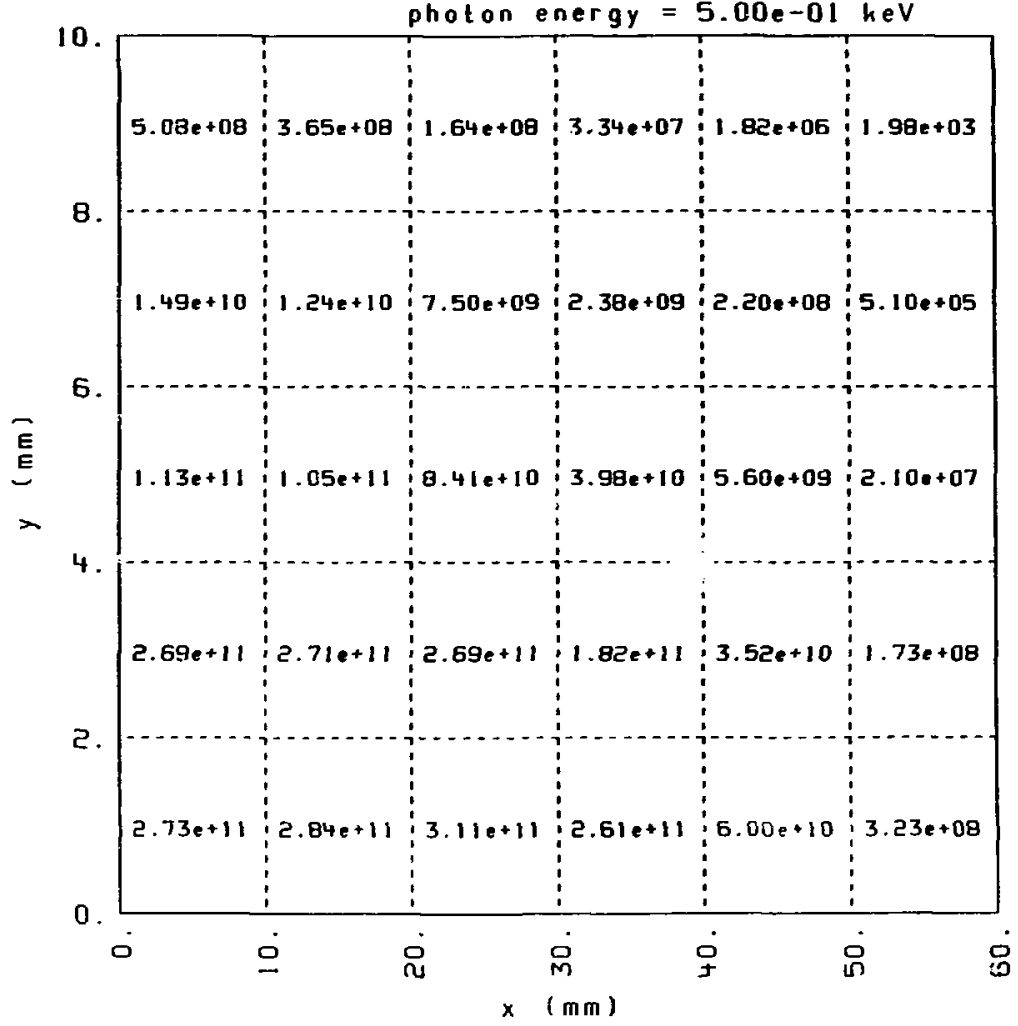

photons/sec/mA in C.Iz bandwidth 
Table Cl.4.

wiggler: 30 poles: $u=13.0 \mathrm{kG} ; 1$ ambda $=12.85 \mathrm{~cm}$ $8.5 \mathrm{~m}$ from center of wiggler ta mirror

electron energy $=1.80 \mathrm{e}+00 \mathrm{GeV}$ critical energy $=2.80 \mathrm{e}+00 \mathrm{keV}$ photon energy $=1.00 \mathrm{e}+00 \mathrm{keV}$

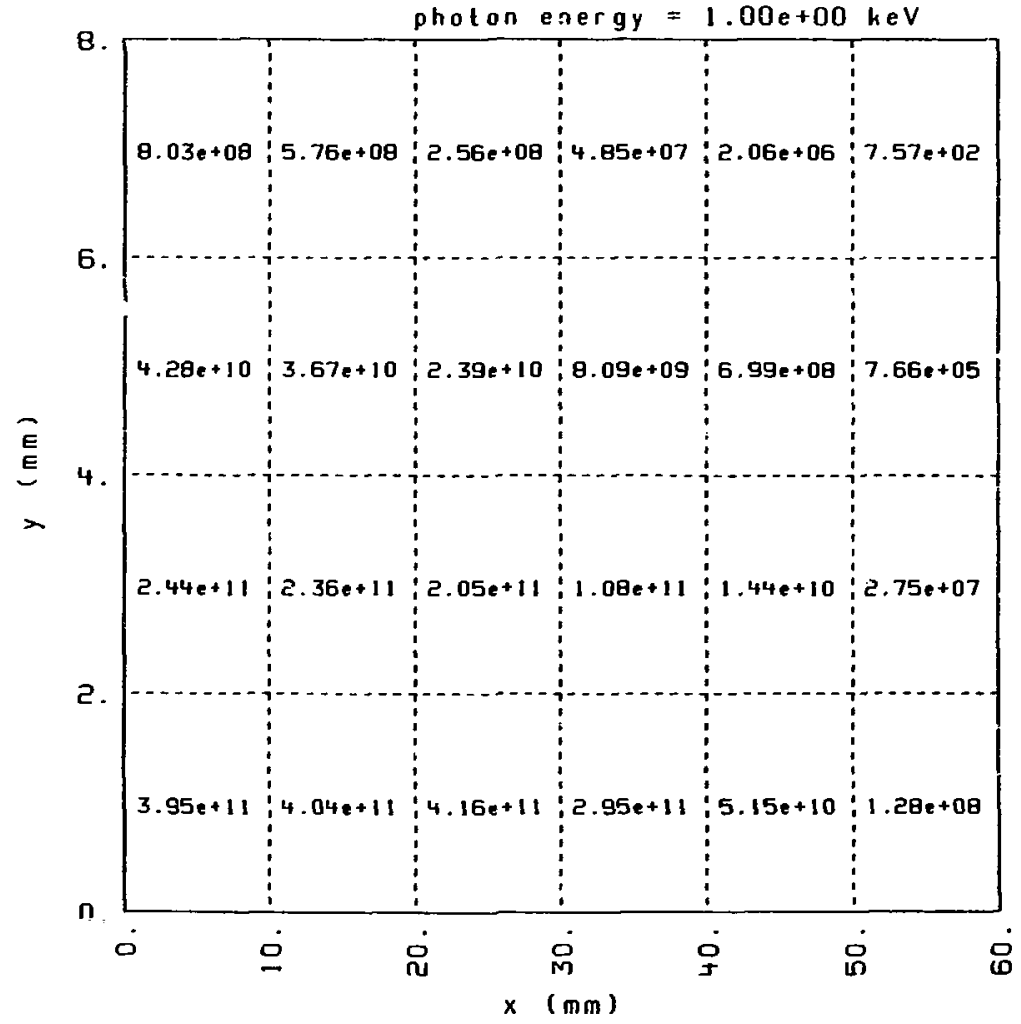

photons/sec/mA in $0.1 x$ bandwidth 
Table C1.5.

wiggler: 30 poles: $B-13.0 \mathrm{kG}$; lambda $=12.85 \mathrm{~cm}$ $8.5 \mathrm{~m}$ from center of wiggler to mirror

electron energy $=1.80 \mathrm{e}+00 \mathrm{GeV}$ crilical energy $=2.80 \mathrm{e}+00 \mathrm{keV}$

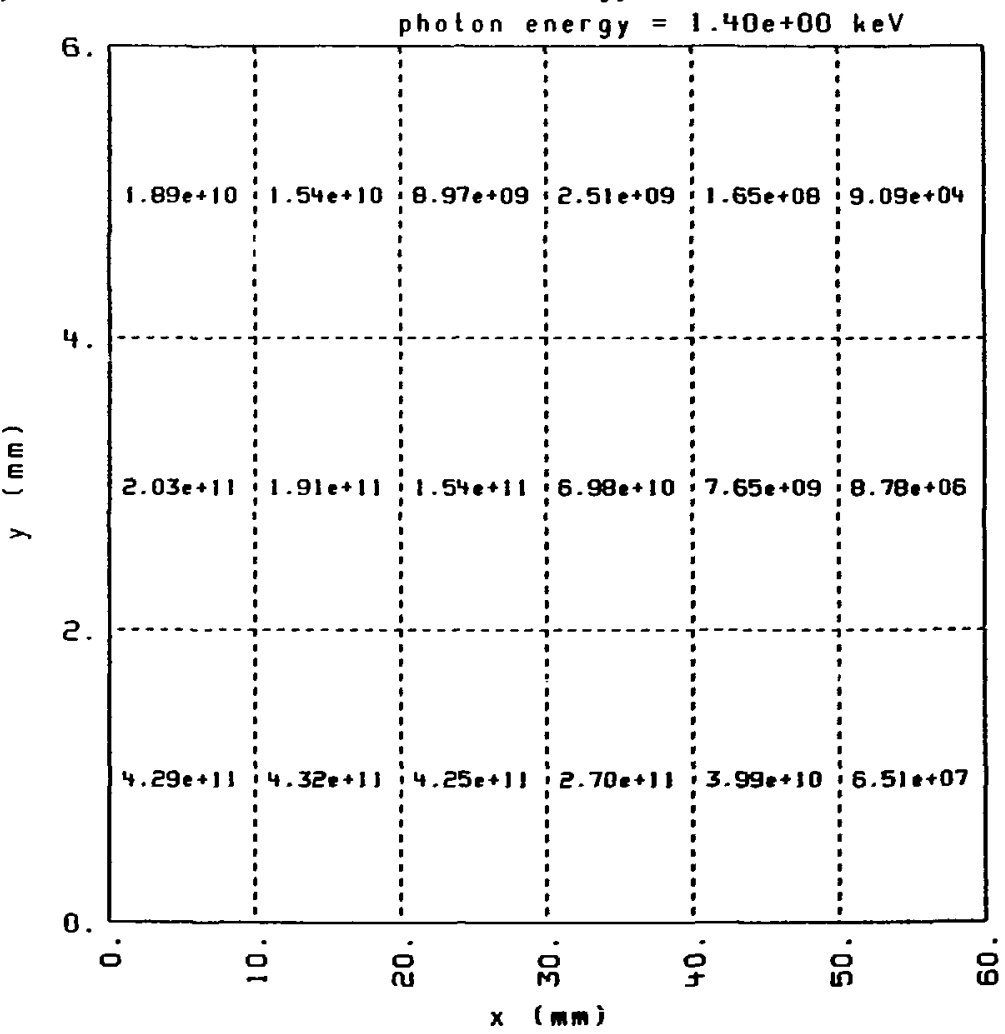

photons/sec/mA in 0.17 bandwidth 
Table C1.6.

wiggler: 30 poles; $B=13.0 \mathrm{kG} ; 1 \mathrm{ambda}=12.85 \mathrm{~cm}$

$8.5 \mathrm{~m}$ from center of wiggler to mirror

electron energy $=1.80 \mathrm{e}+00 \mathrm{GeV}$ critical energy $=2.80 \mathrm{l}+00 \mathrm{keV}$

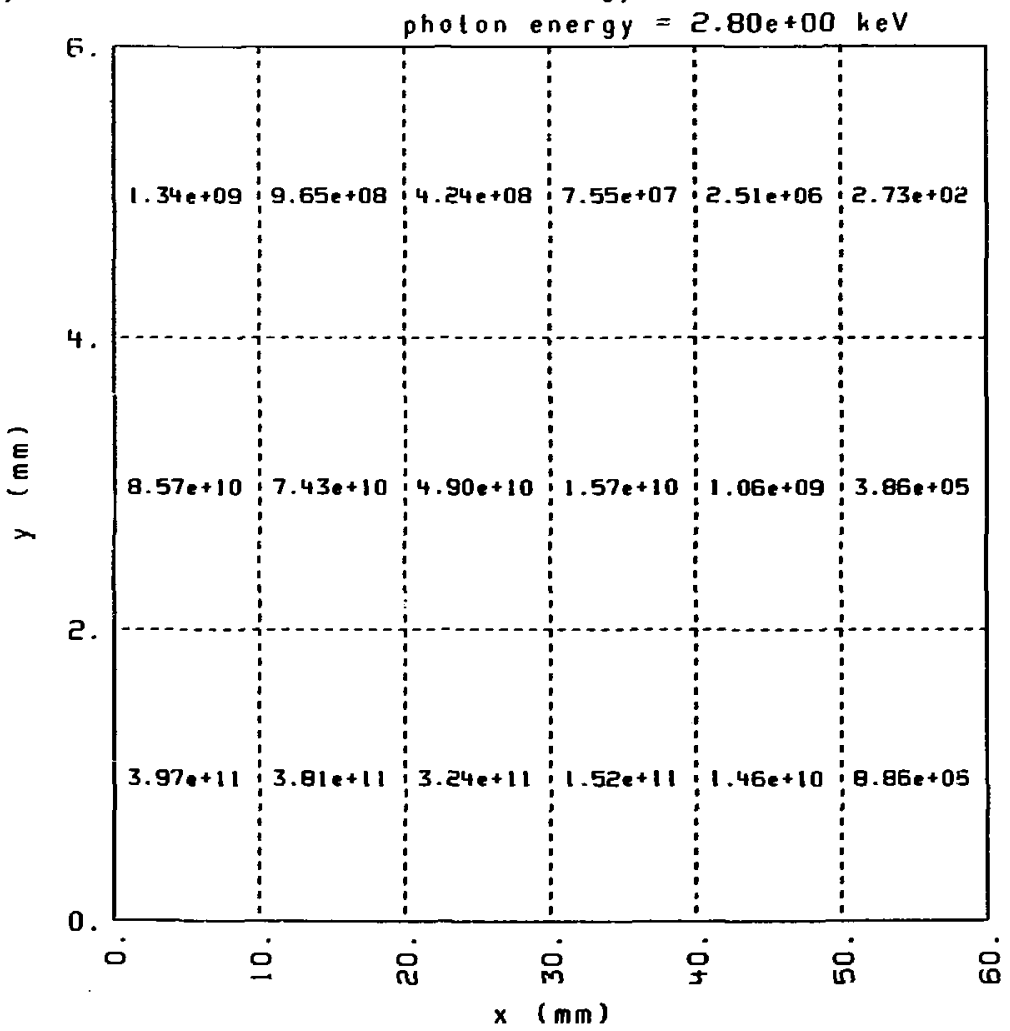

pholons/sec/mA in $0.1 x$ bandwidth 
Table C1.7.

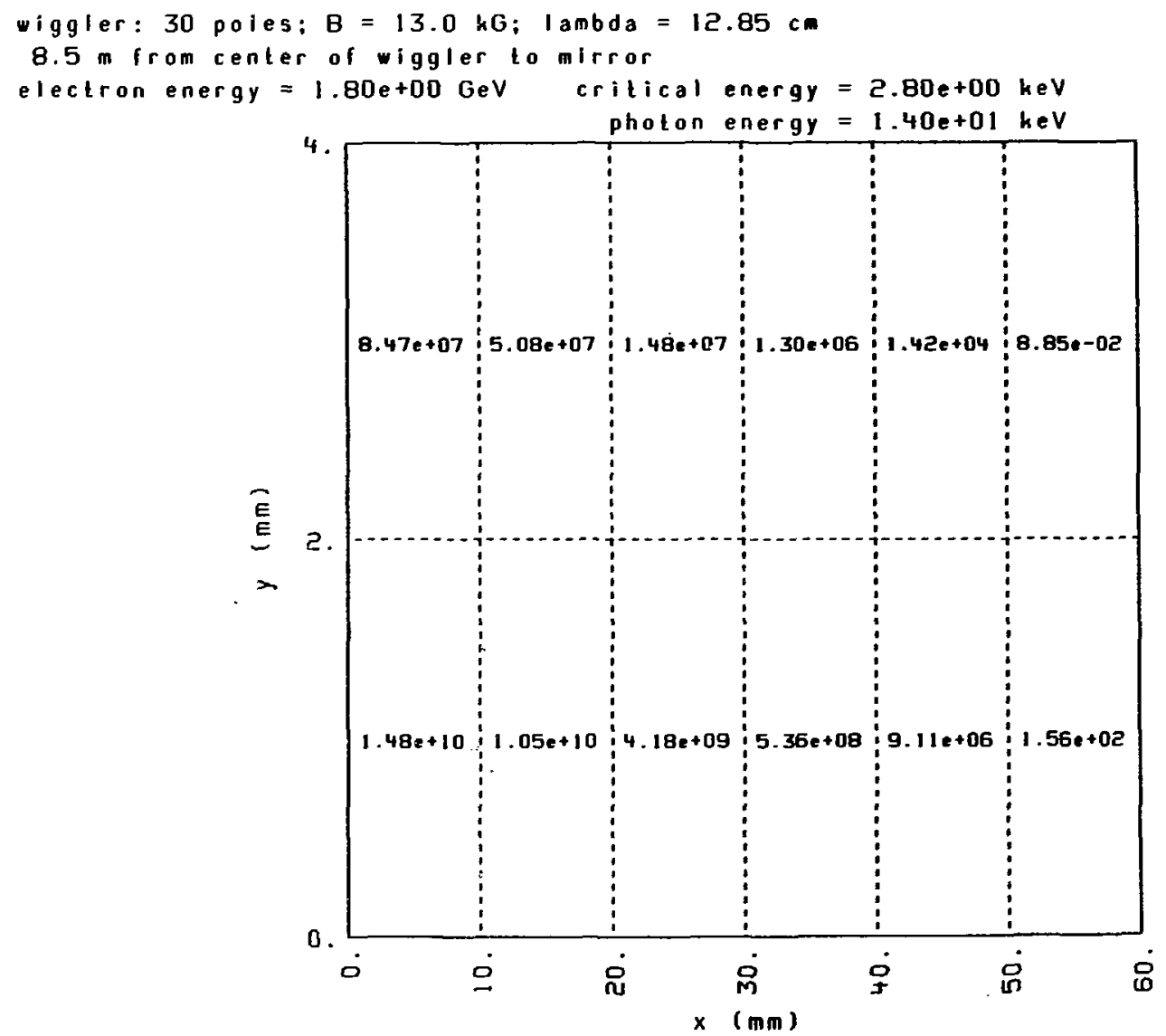

photons/sec/mA in $0.1 \%$ bandwidth 
Table C2.1.

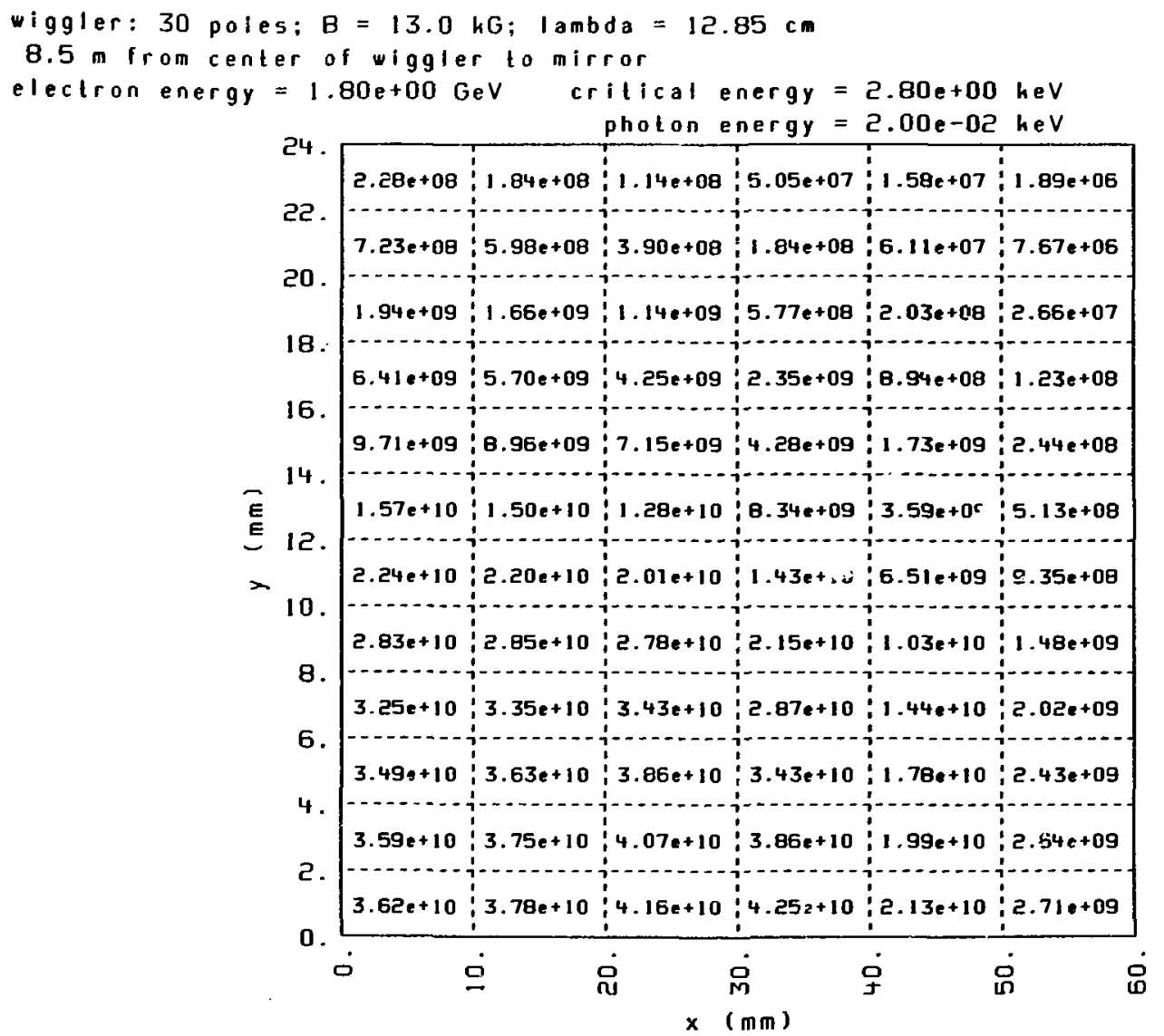

paral le I

polarization

photons/sec/mA in $0.1 \%$ bandwidth 
Table C2.2.

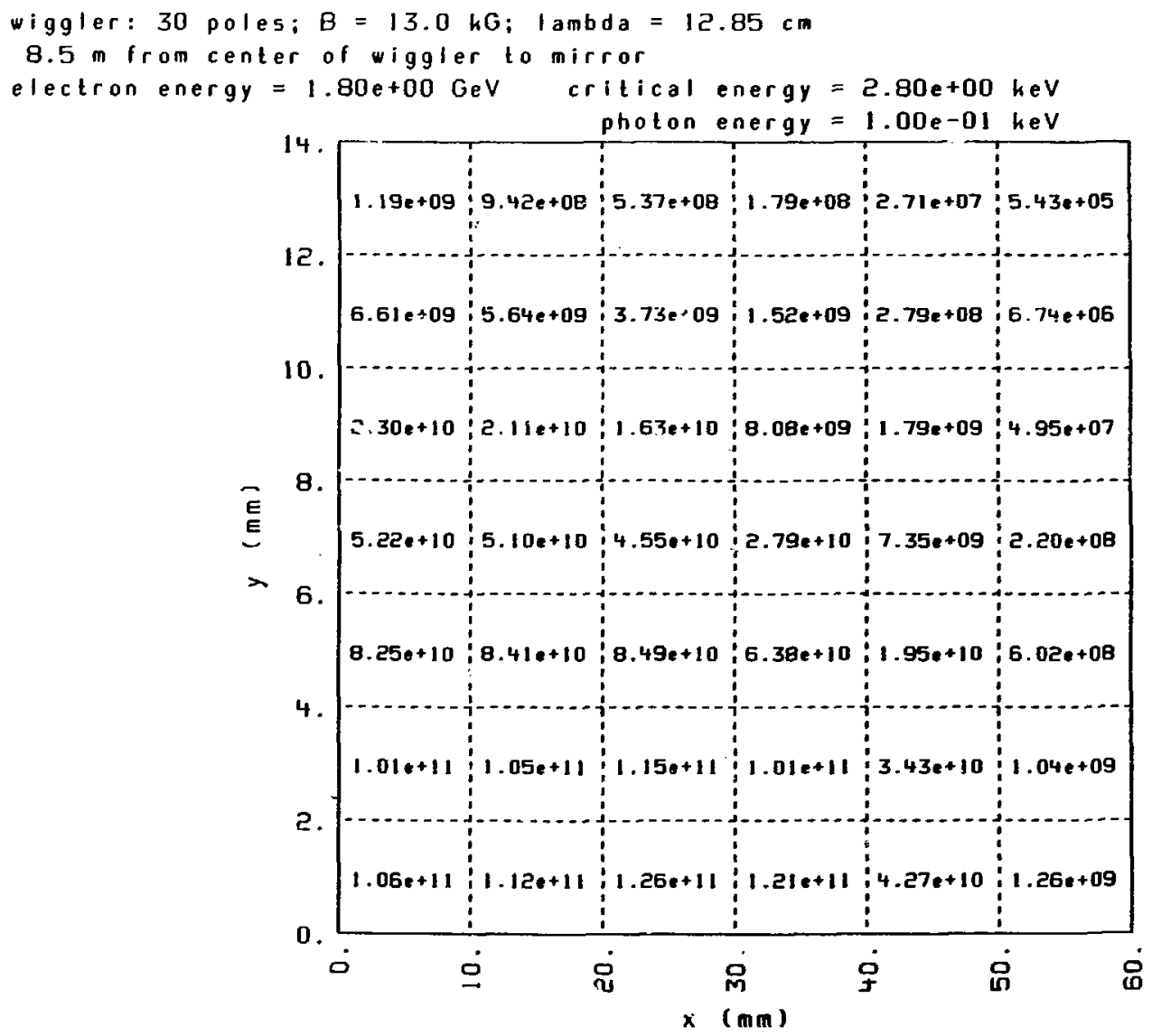

parallel

polarization

photons/sec/mA in $0.1 \%$ bandwidth 
Table C2.3.

wiggler: 30 poles; $B=13.0 \mathrm{kG} ; \mathrm{Iambda}=12.85 \mathrm{~cm}$

$8.5 \mathrm{~m}$ from center of wiggler to mirror

electron energy $=1.80 \mathrm{e}+00 \mathrm{GeV}$ crilical energy $=2.80 \mathrm{e}+00 \mathrm{heV}$

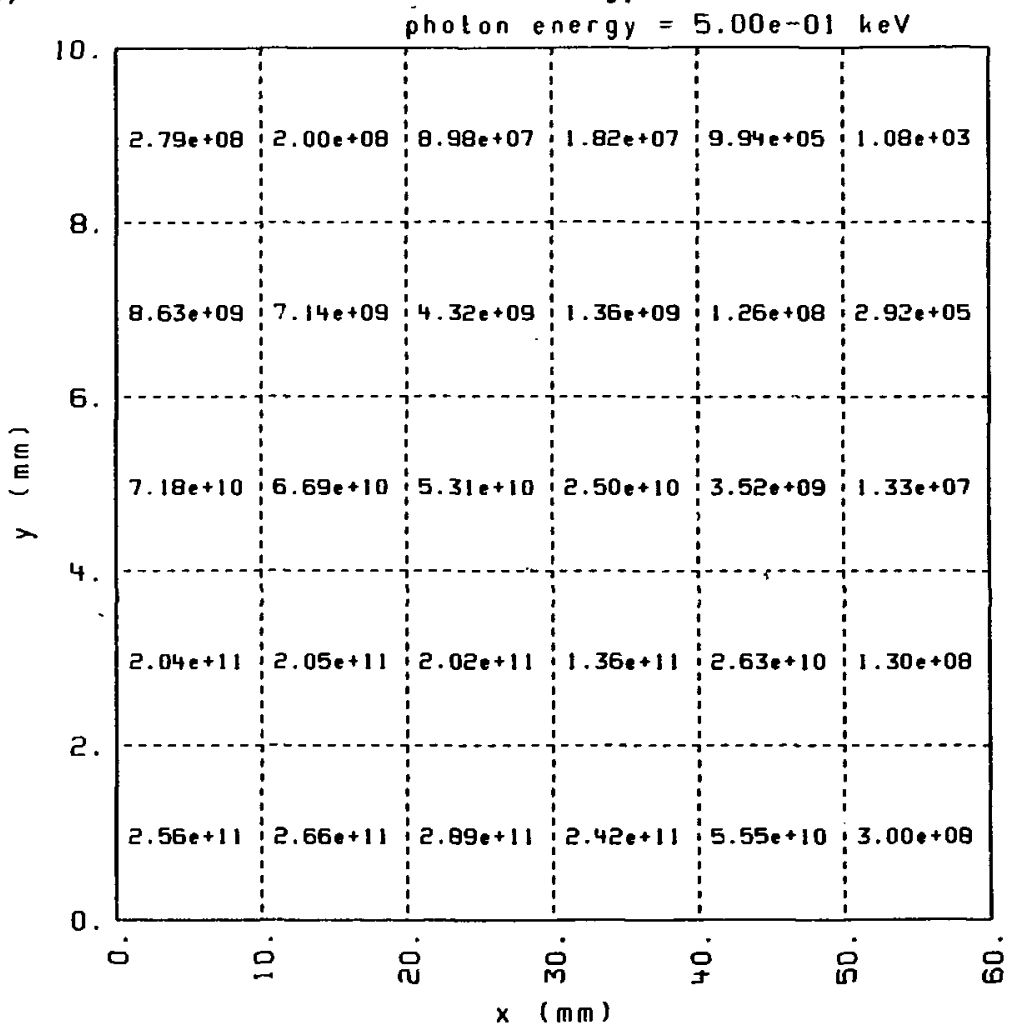

parallel

polarization

photons/sec/mA in $0.1 *$ bandwidth 
Table C2.4.

wiggler: 30 poles: $B=13.0 \mathrm{hG} ; \mathrm{lambda}=12.85 \mathrm{~cm}$ 3.5 irom center of wiggler to mirror

electron energy $=1.80 \mathrm{e}+00 \mathrm{GeV}$ critical energy $=2.80 \mathrm{e}+00 \mathrm{heV}$ photon energy $=1.00 \mathrm{e}+00 \mathrm{heV}$

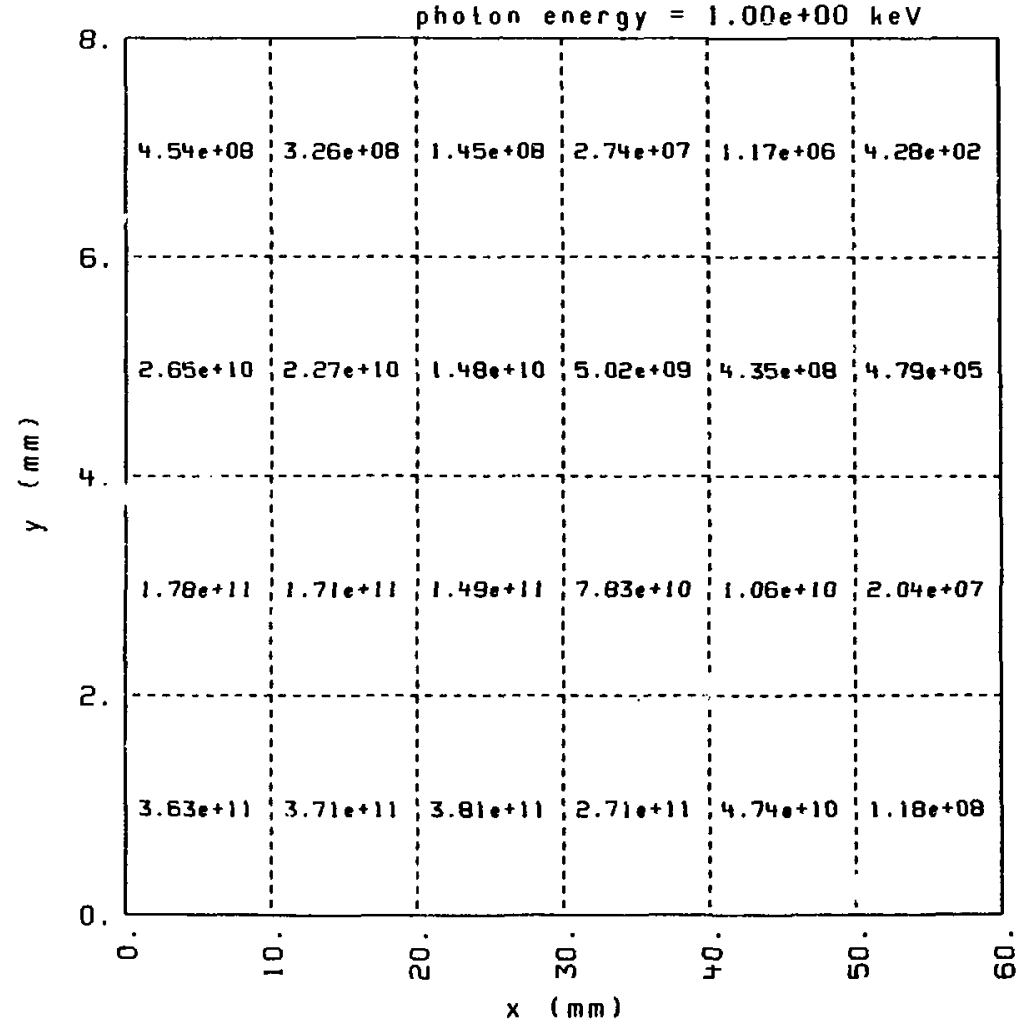

parallel

polartzation

photons/secima in $0.1 x$ bandwidth 
Table C2.5.

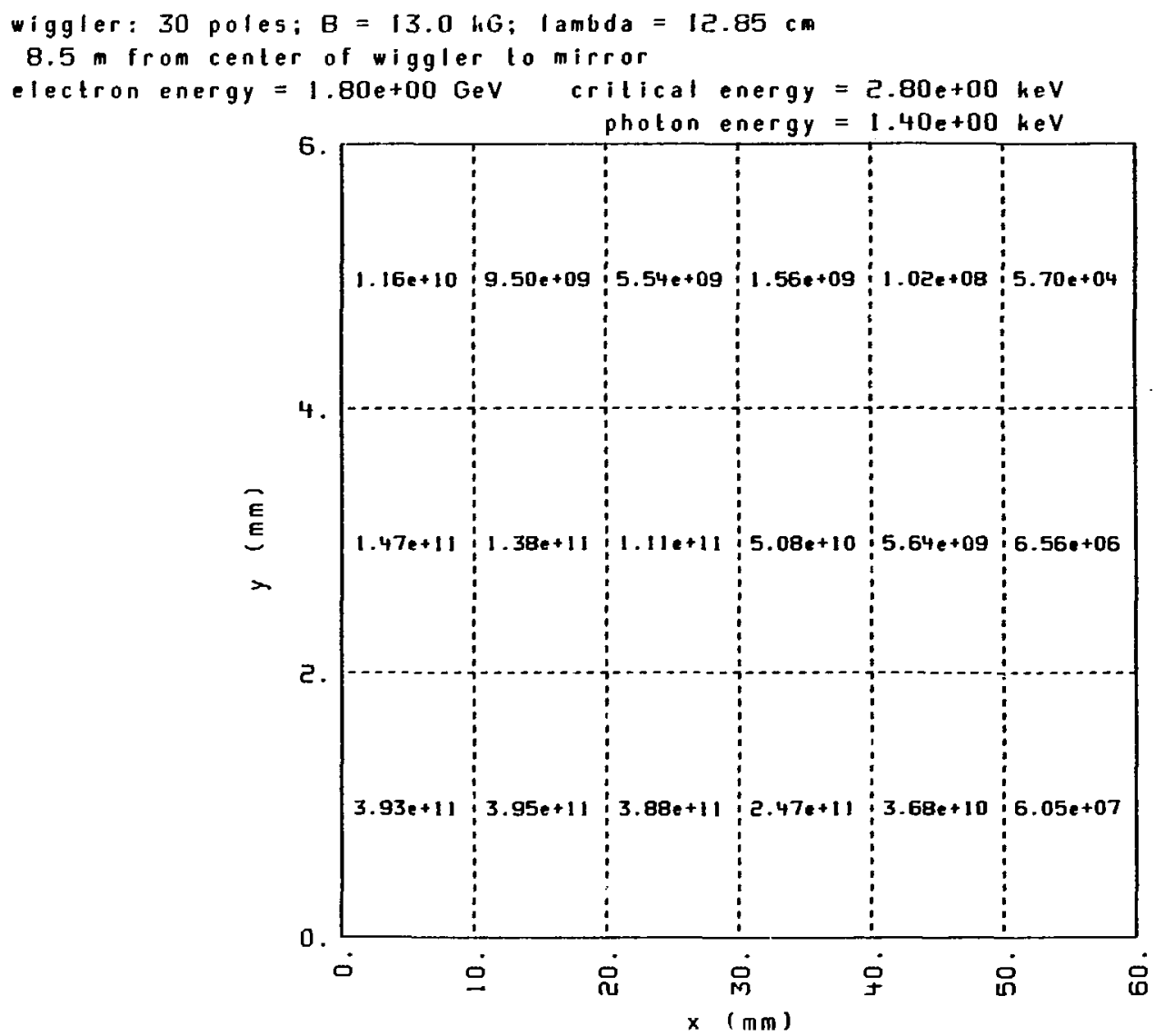

paralle I

polarization 
Table C2.6.

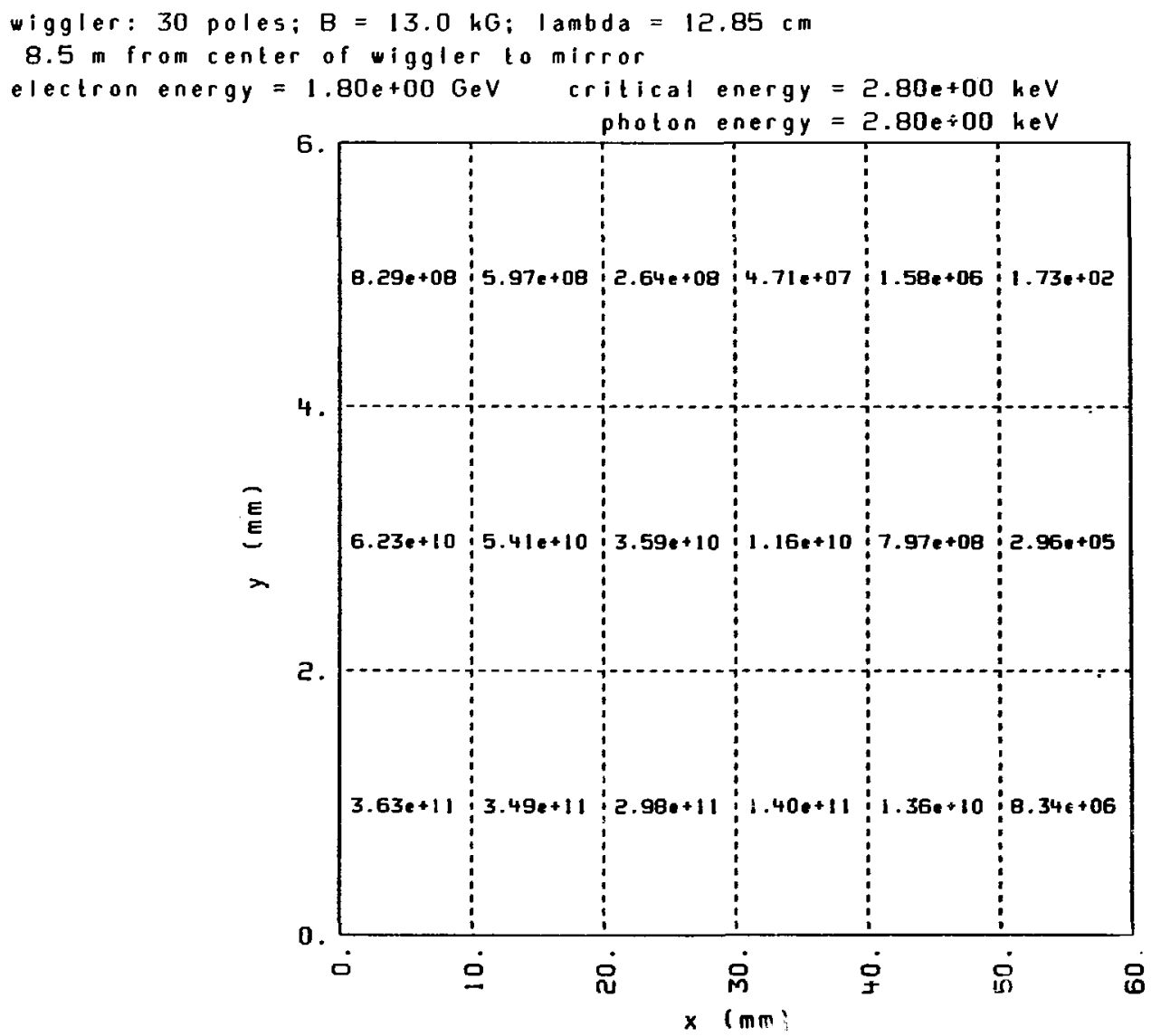

parallel

polarization

pholons/ser./mA in $0.1 \%$ bandwidth 
Table C2.7.

wiggler: 30 poles; $B=13.0 \mathrm{kG} ; \mathrm{lambda}=12.85 \mathrm{~cm}$ $8.5 \mathrm{~m}$ from center of wiggler to mirror electron energy $=1.80 e+00 \mathrm{GeV}$ critical energy $=2.80 e+00 \mathrm{keV}$ photon energy $=1.40 \mathrm{e}+01 \mathrm{keV}$

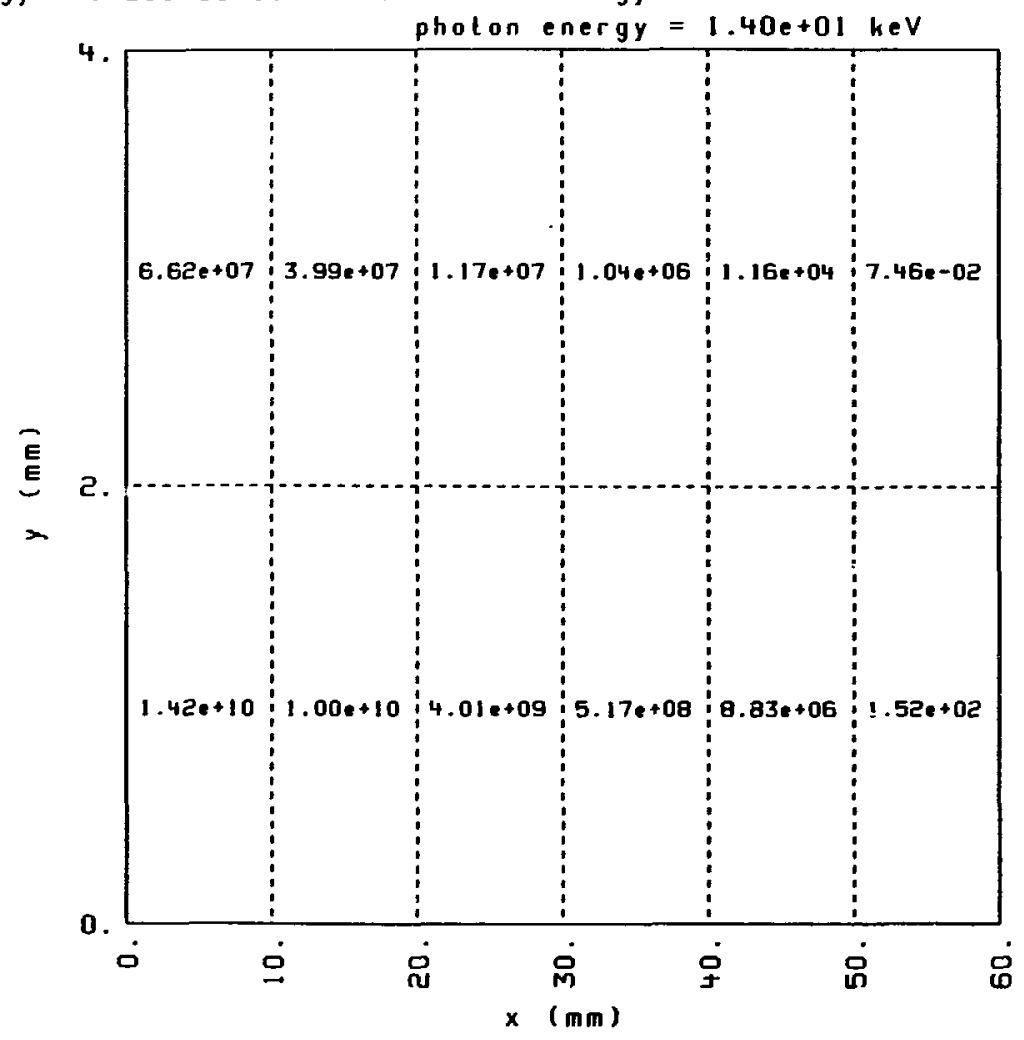


Table C3.1.

wiggler: 30 poles: $B=13.0 \mathrm{kG} ; \mathrm{lambda}=12.85 \mathrm{~cm}$ $8.5 \mathrm{~m}$ from center of wiggler to mirror

electron energy $=1.80 \mathrm{e}+00 \mathrm{GeV}$ critical energy $=2.80 \mathrm{e}+00 \mathrm{heV}$ photon energy $=2.00 e-02$ heV

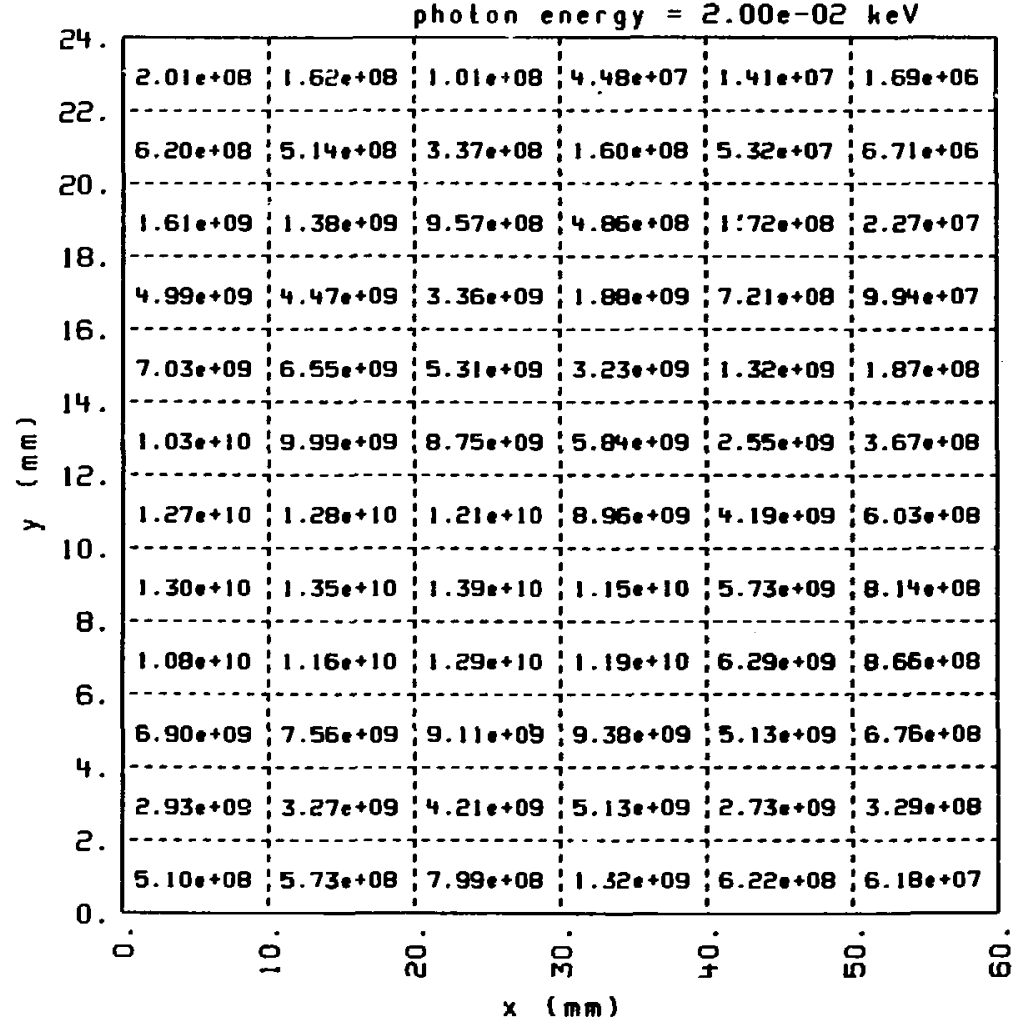

perpendicular polarization 
Table C3.2.

wiggler: 30 poles: $B=13.0 \mathrm{kG}: \operatorname{Iambda}=12.85 \mathrm{~cm}$ $8.5 \mathrm{~m}$ from center of wiggler to mirror electron energy $=1.80 \mathrm{e}+00 \mathrm{GeV}$ critical energy $=2.80 \mathrm{e}+00 \mathrm{heV}$

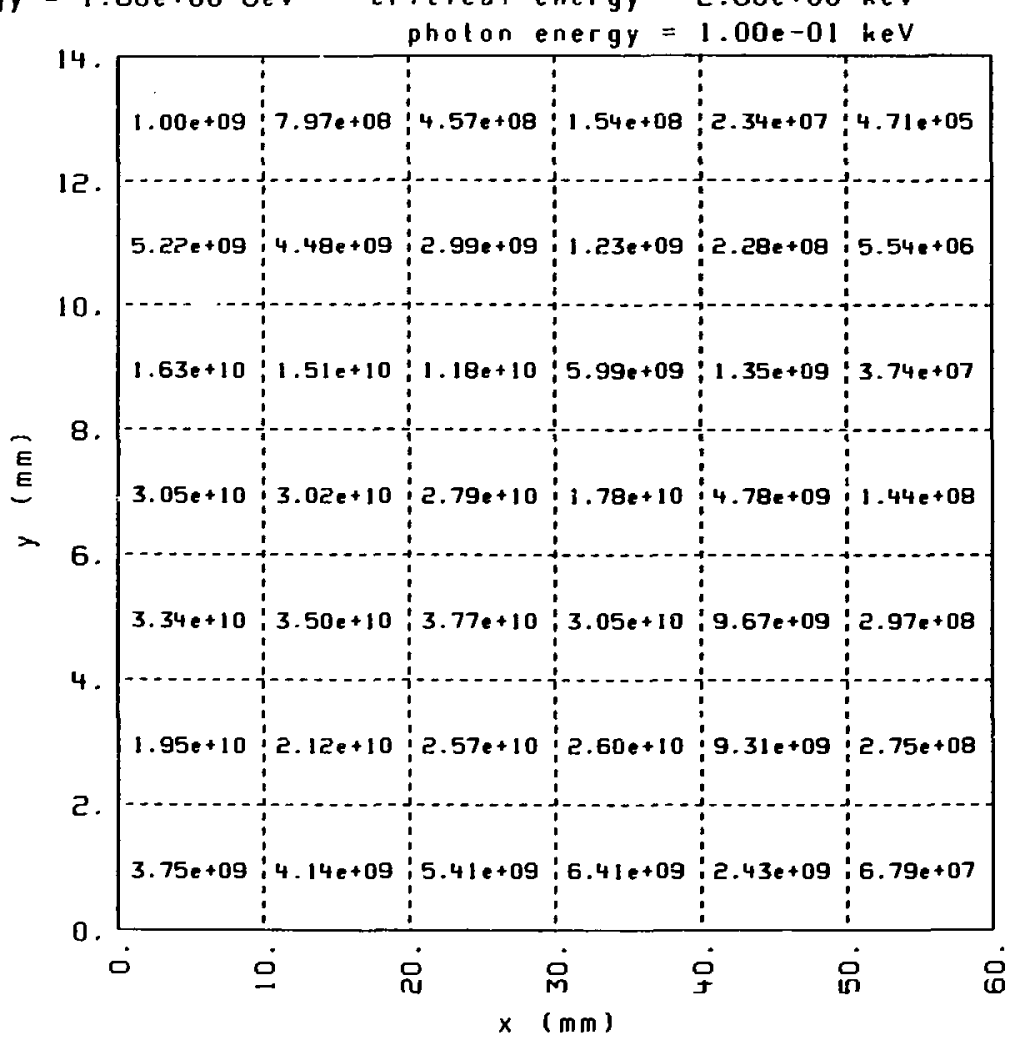

perpendicular

polarization 
Table C3.3.

wiggler: 30 poles; $B=13.0 \mathrm{hG} ; 1$ ambda $=12.85 \mathrm{~cm}$ $8.5 \mathrm{~m}$ from center of wiggler to mirror

electron energy $=1.80 \mathrm{e}+00 \mathrm{GeV}$ critical energy $=2.80 \mathrm{e}+00 \mathrm{heV}$

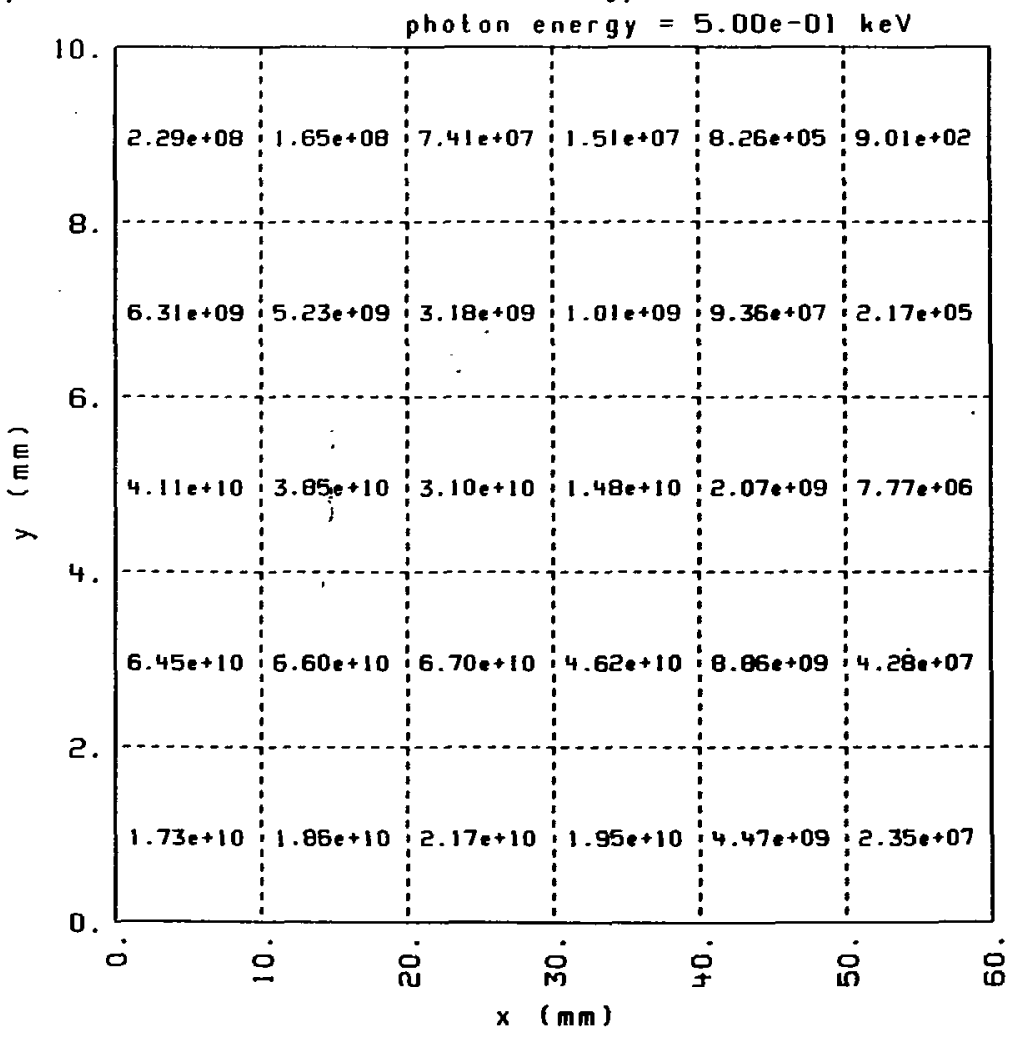

perpendicular

polarization

photons/sec/mA in $0.1 \%$ bandwidth 
Táble C3.4.

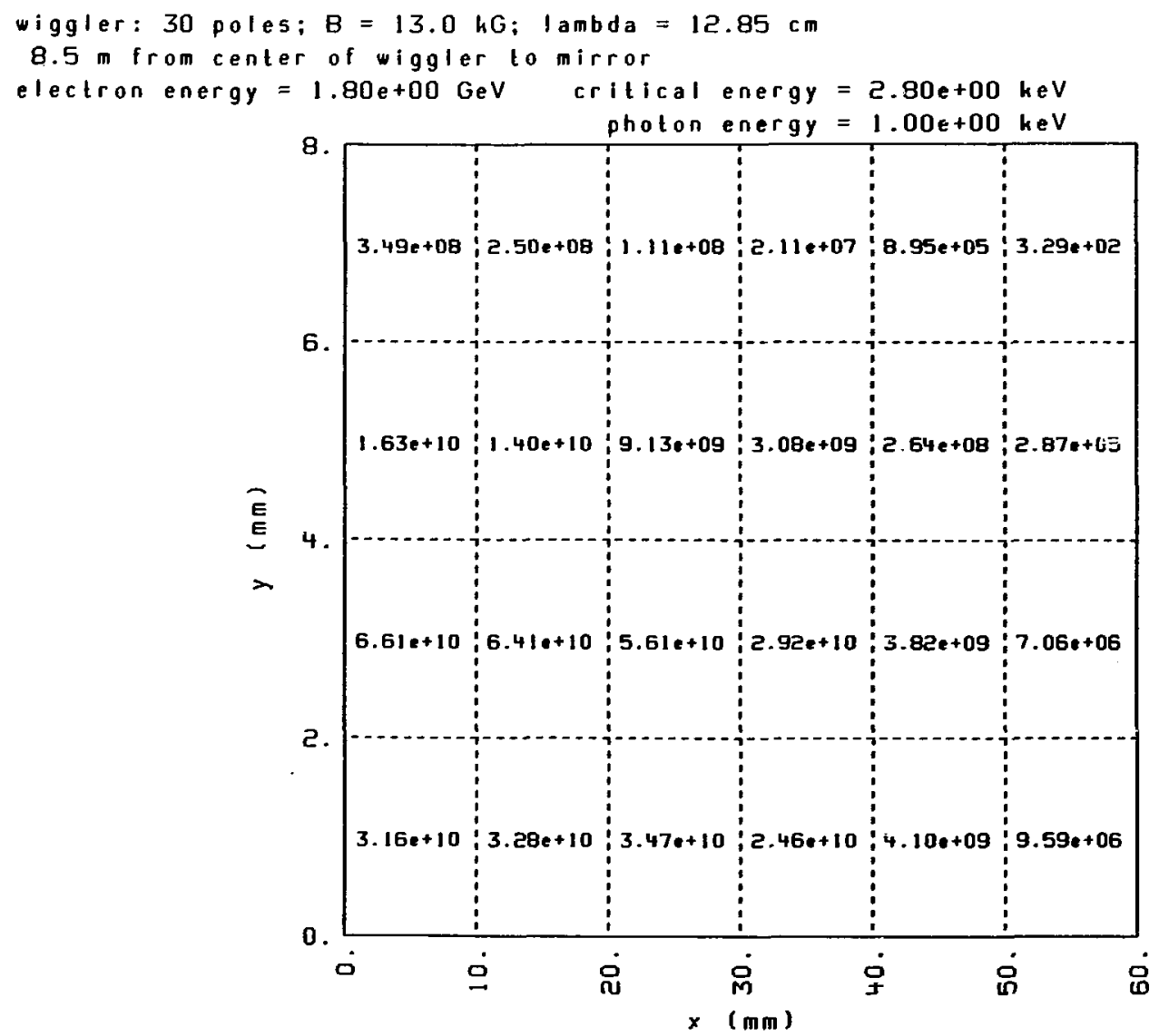

perpendicular

polarization

photons/sec/mA in $0.1 x$ bandwidth 


\section{Table C3.5.}

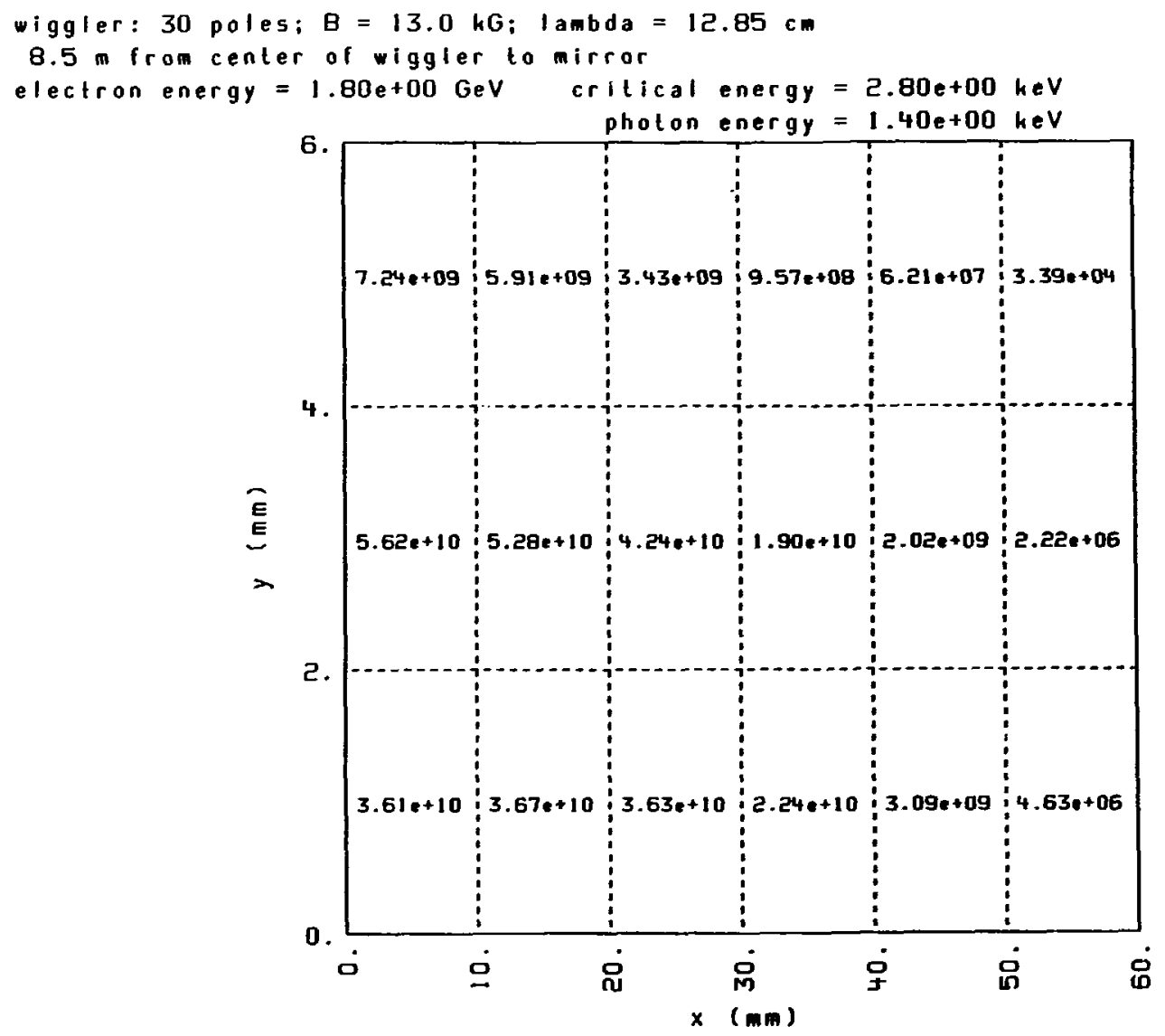

perpendicular polarization 
Table C3.6.

wiggler: 30 poles; $B=13.0 \mathrm{kG} ; \mathrm{Iambda}=12.85 \mathrm{~cm}$ $8.5 \mathrm{~m}$ from center of wiggler to mirror

electron energy $=1.80 \mathrm{e}+00 \mathrm{GeV}$ critical energy $=2.80 \mathrm{e}+00 \mathrm{keV}$ photon energy $=2.80 \mathrm{e}+00 \mathrm{keV}$

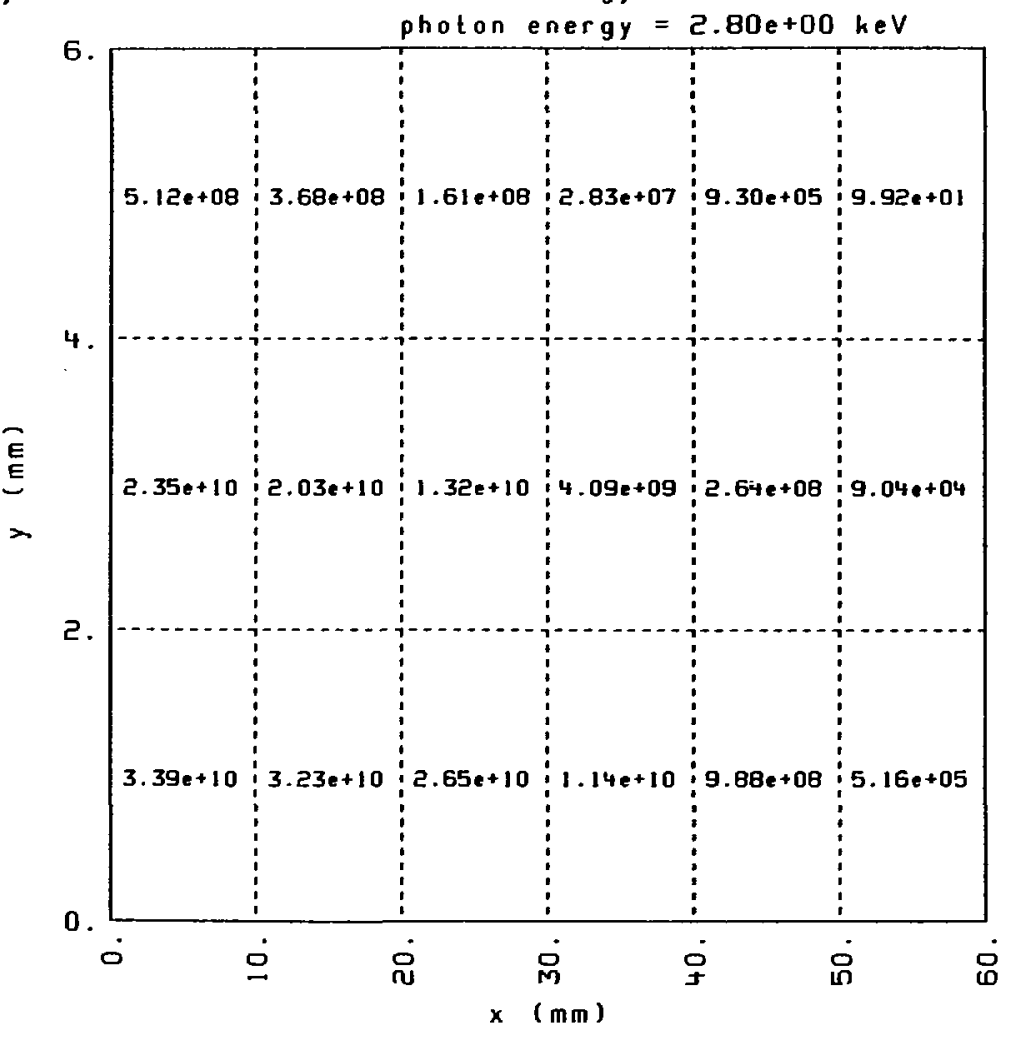

perpendicular polarization 
Table C3.7.

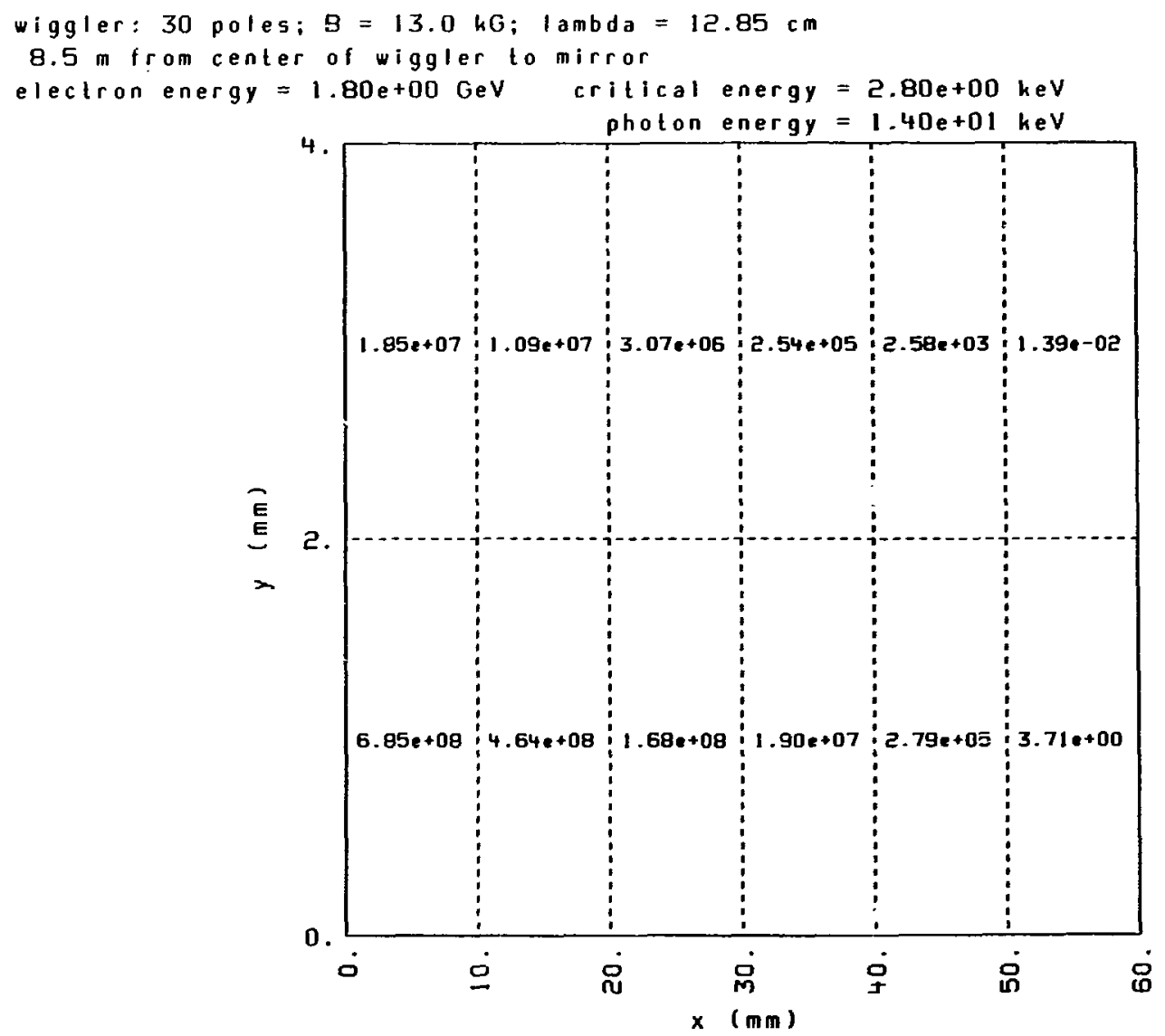

perpendicular

polarization

photons/sec/mA in $0.1 x$ bandwidth 
Table C4.I.

wiggler: 30 poles; $B=13.0 \mathrm{kG} ; 1$ ambda $=12.85 \mathrm{~cm}$

$8.5 \mathrm{~m}$ from center of wigler to mirror

elactron energy $=3.00 \mathrm{e}+00 \mathrm{GeV}$ critical energy $=7.78 \mathrm{e}+00 \mathrm{keV}$

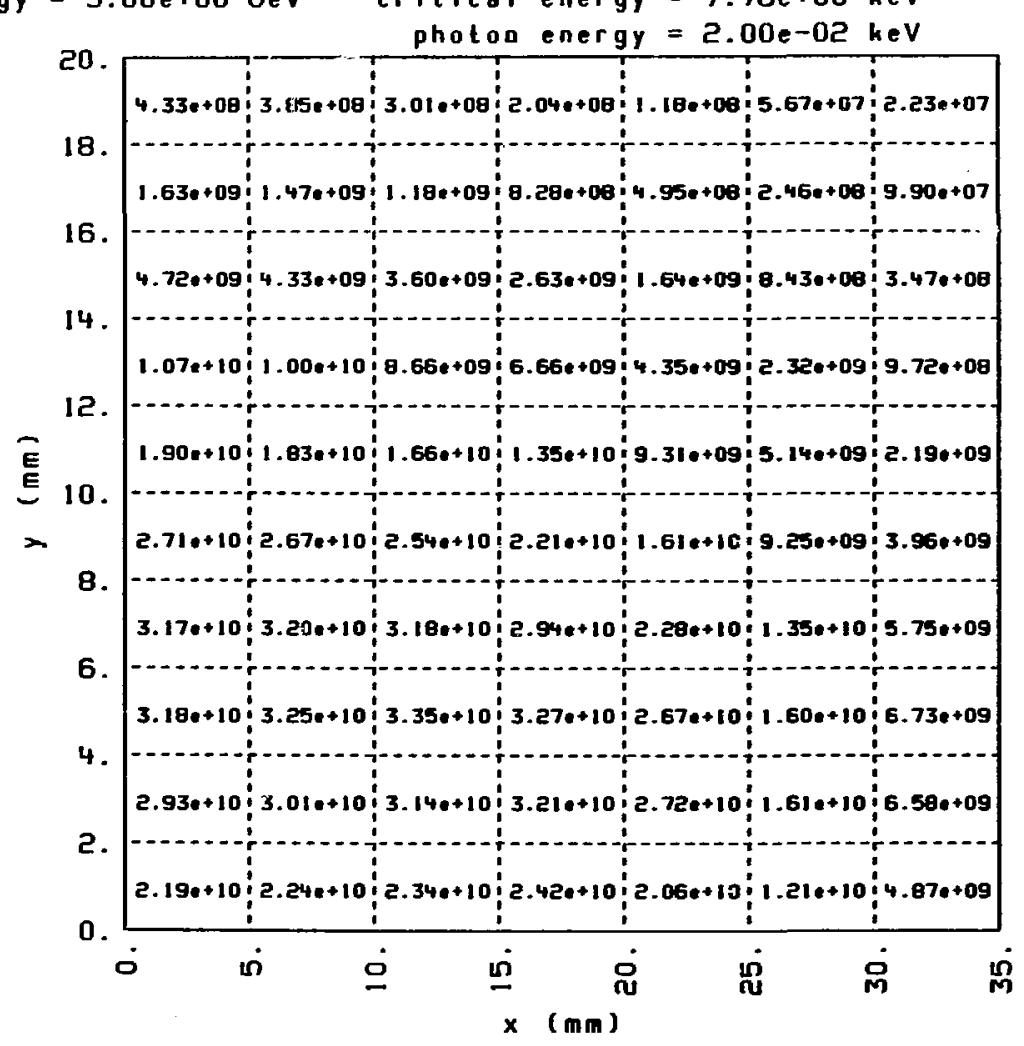

photons/sec/mA in $0.1 \%$ bandwidth 
Table C4.2.

wigler: 30 poles: $B=13.0 \mathrm{hG} ; \mathrm{Iambda}=12.85 \mathrm{~cm}$

$8.5 \mathrm{~m}$ from center of wiggler to mirror

electron energy $=3.00 \mathrm{e}+00 \mathrm{GeV}$ critical energy $=7.78 \mathrm{e}+00 \mathrm{keV}$

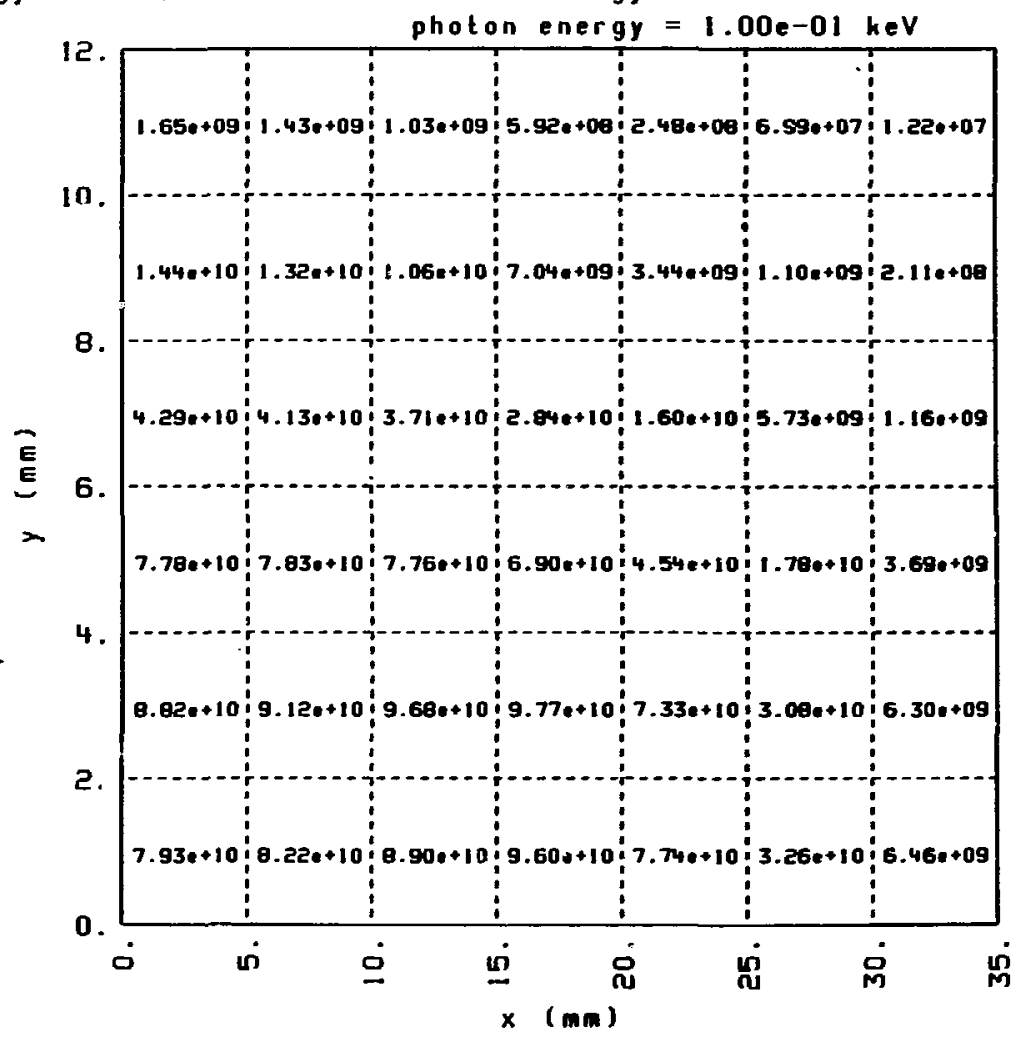

photons/sec/mA in 0.17 bandwidth 
Table C4.3.

wiggler: 30 poles: $B=13.0 \mathrm{kG} ; \mathrm{Iambda}=12.85 \mathrm{~cm}$ $8.5 \mathrm{~m}$ from center of wiggler to mirror

electron energy $=3.00 \mathrm{e}+00 \mathrm{GeV} \quad \mathrm{critical}$ energy $=7.78 \mathrm{e}+00 \mathrm{keV}$ photon energy $=5.00 \mathrm{e}-01 \mathrm{keV}$

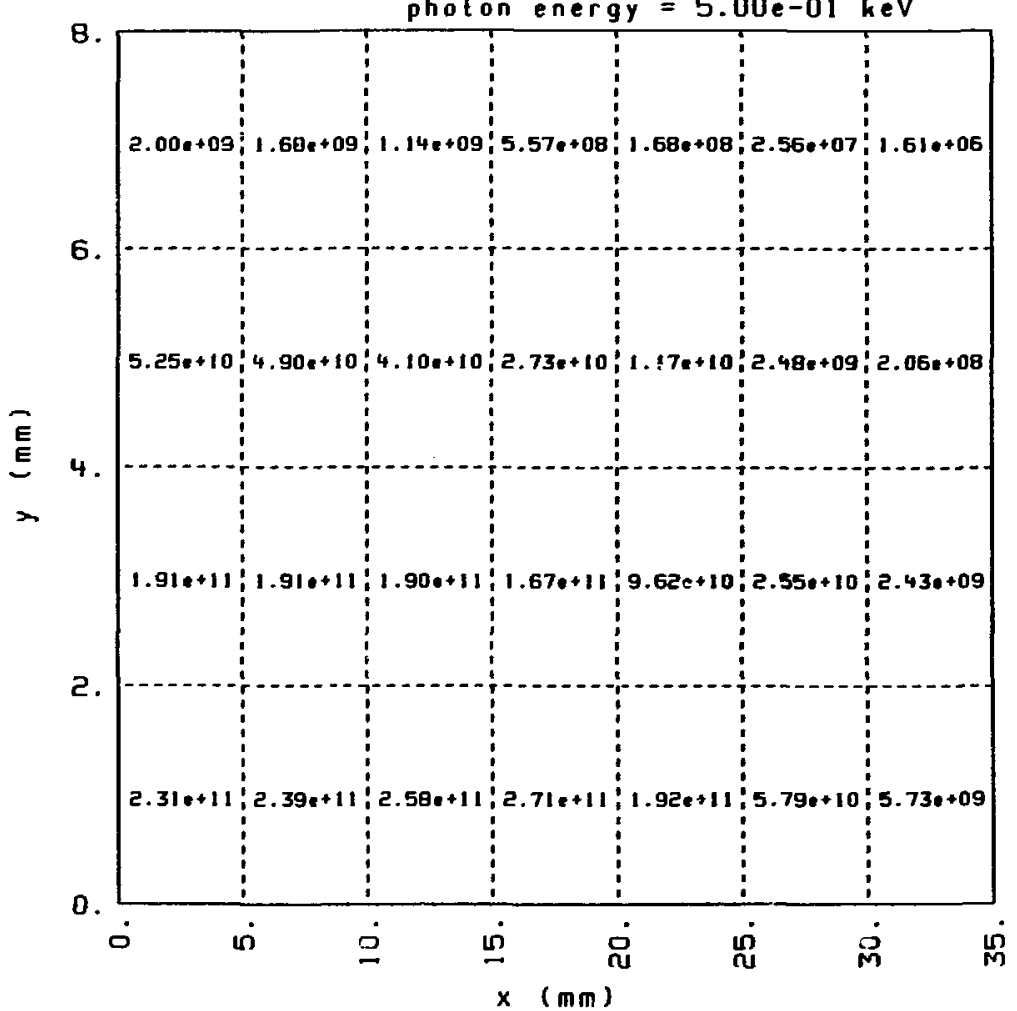

photons/sec/mA in $0.1 \%$ bandwidth 
Table C4.4.

wiggler: 30 poles; $B=13.0 \mathrm{hG} ; 1$ ambda $=12.85 \mathrm{~cm}$ $8.5 \mathrm{~m}$ from center of wiggler to mirror

electron energy $=3.00 \mathrm{e}+00 \mathrm{GeV}$ critical energy $=7.78 \mathrm{e}+00 \mathrm{keV}$

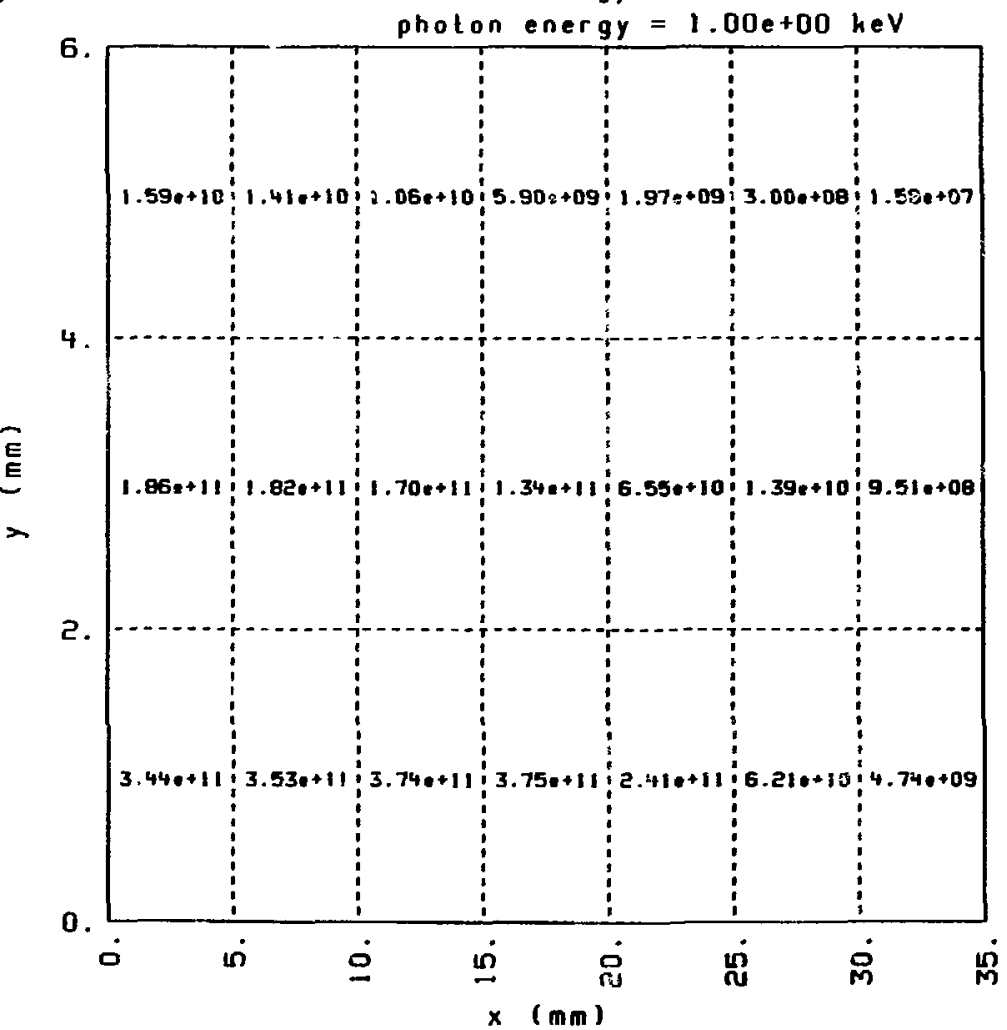

photons/sec/ma in $0.1 x$ bandwidth 
Table C4.5.

wigler: 30 poles; $B=13.0 \mathrm{kG} ;$ lambda $=12.85 \mathrm{~cm}$

8.5 from center of wiggler to inirror

electran energy $=3.00 \mathrm{e}+00 \mathrm{GeV}$ critical energy $=7.78 \mathrm{e}+00 \mathrm{keV}$

photon energy $=3.89 e+00 \mathrm{heV}$

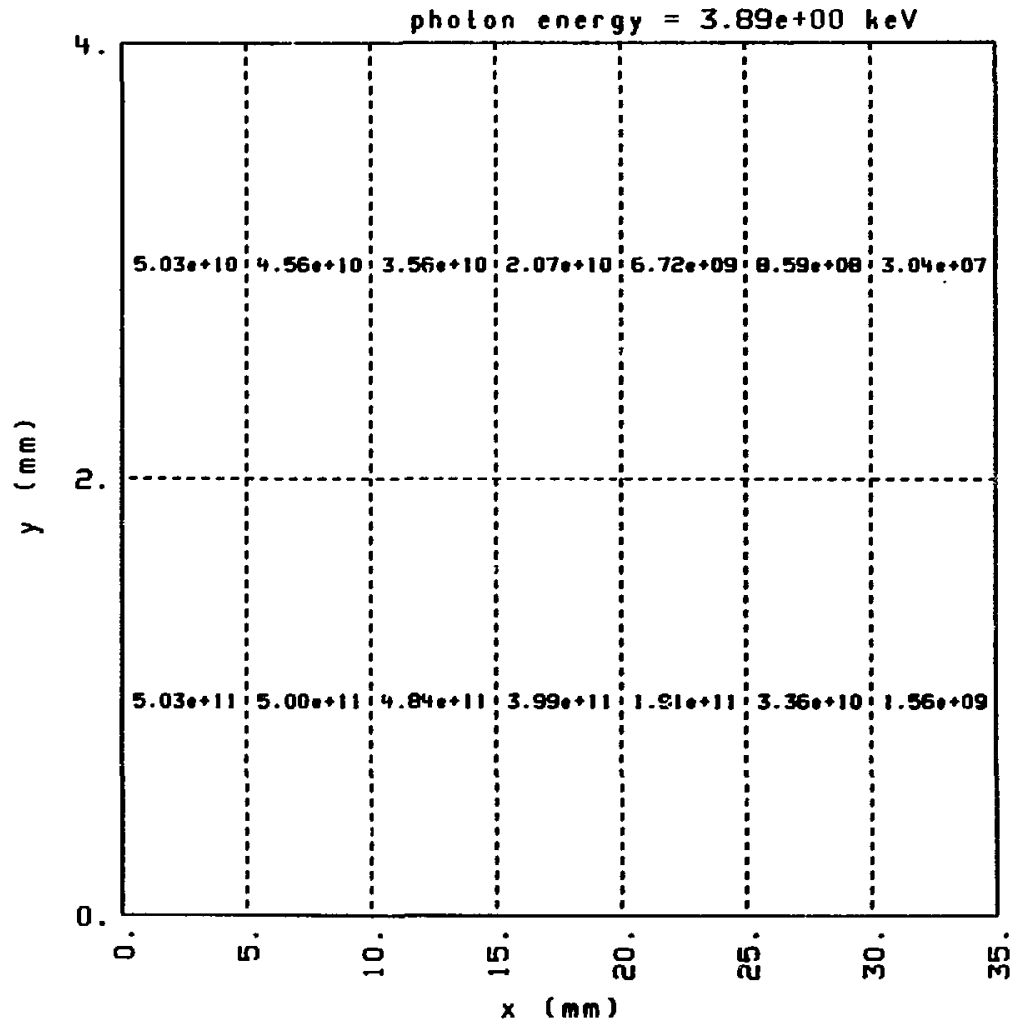

photons/sec/mA in $0.1 \%$ bandeidth 
Table C4.6.

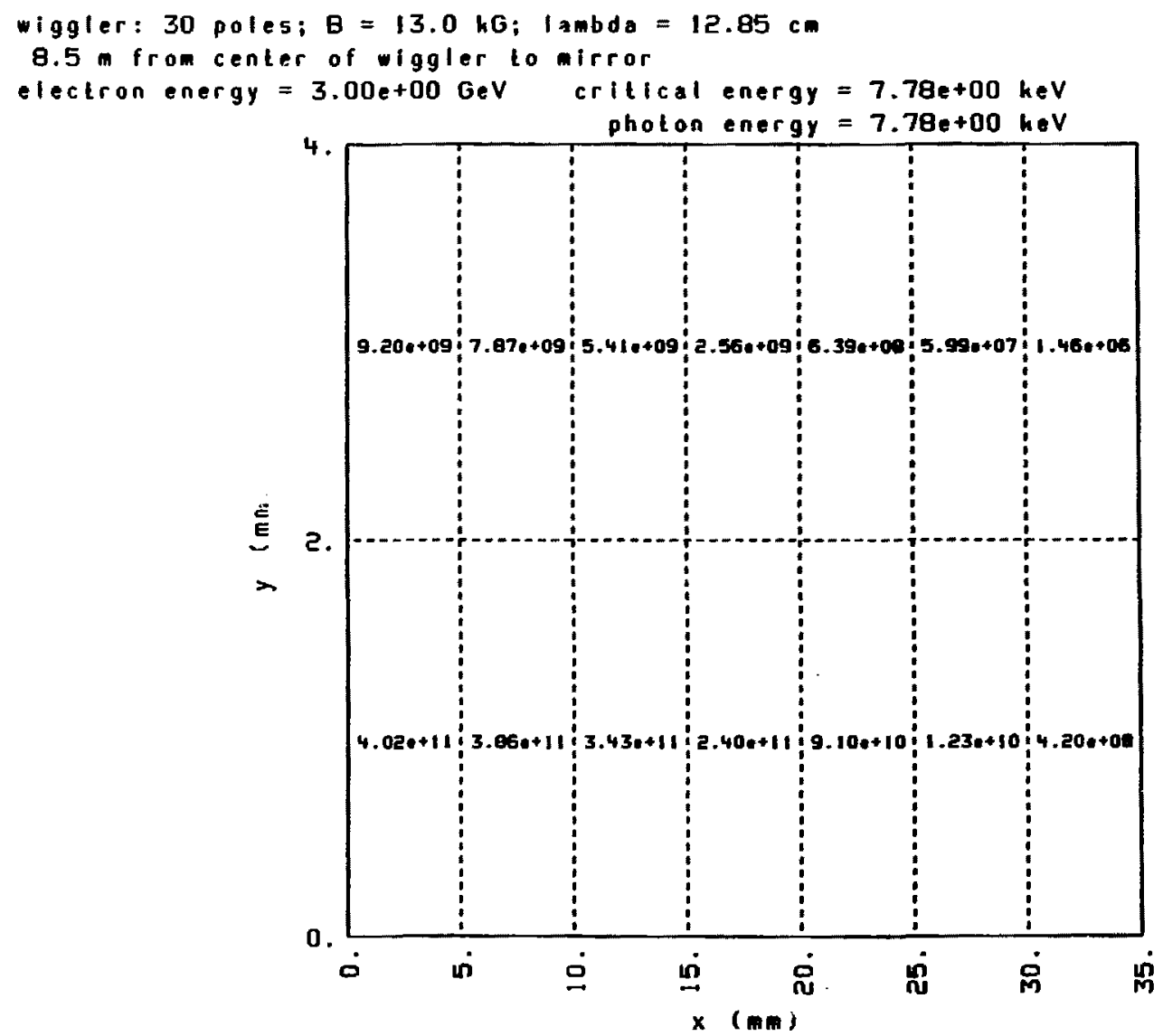

photons/sec/mA in $0.1 \%$ bandwidth 


\section{Table C4.7.}

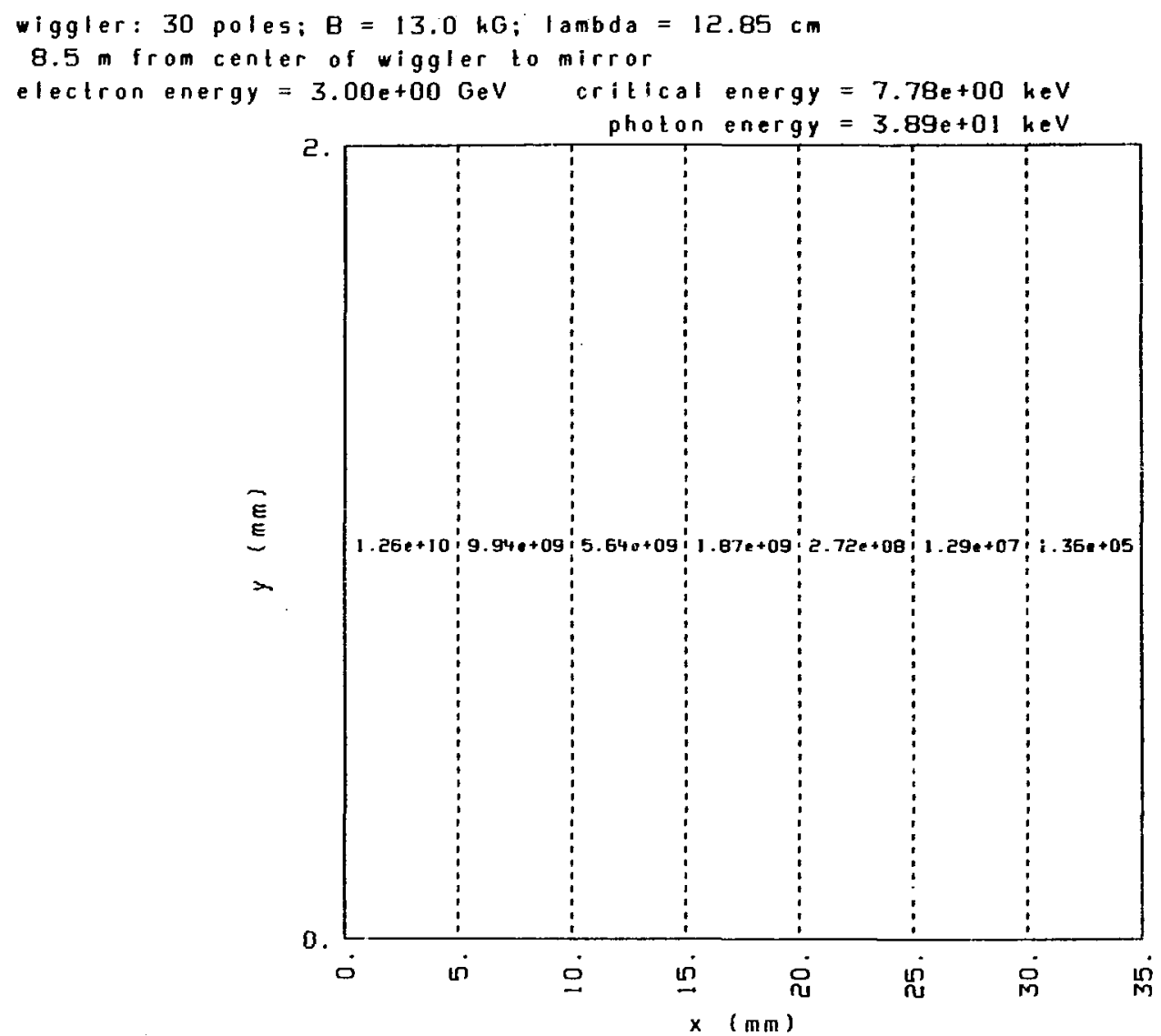

photons/sec/mA in $0.1 \%$ bandwidth 
Table C5.1.

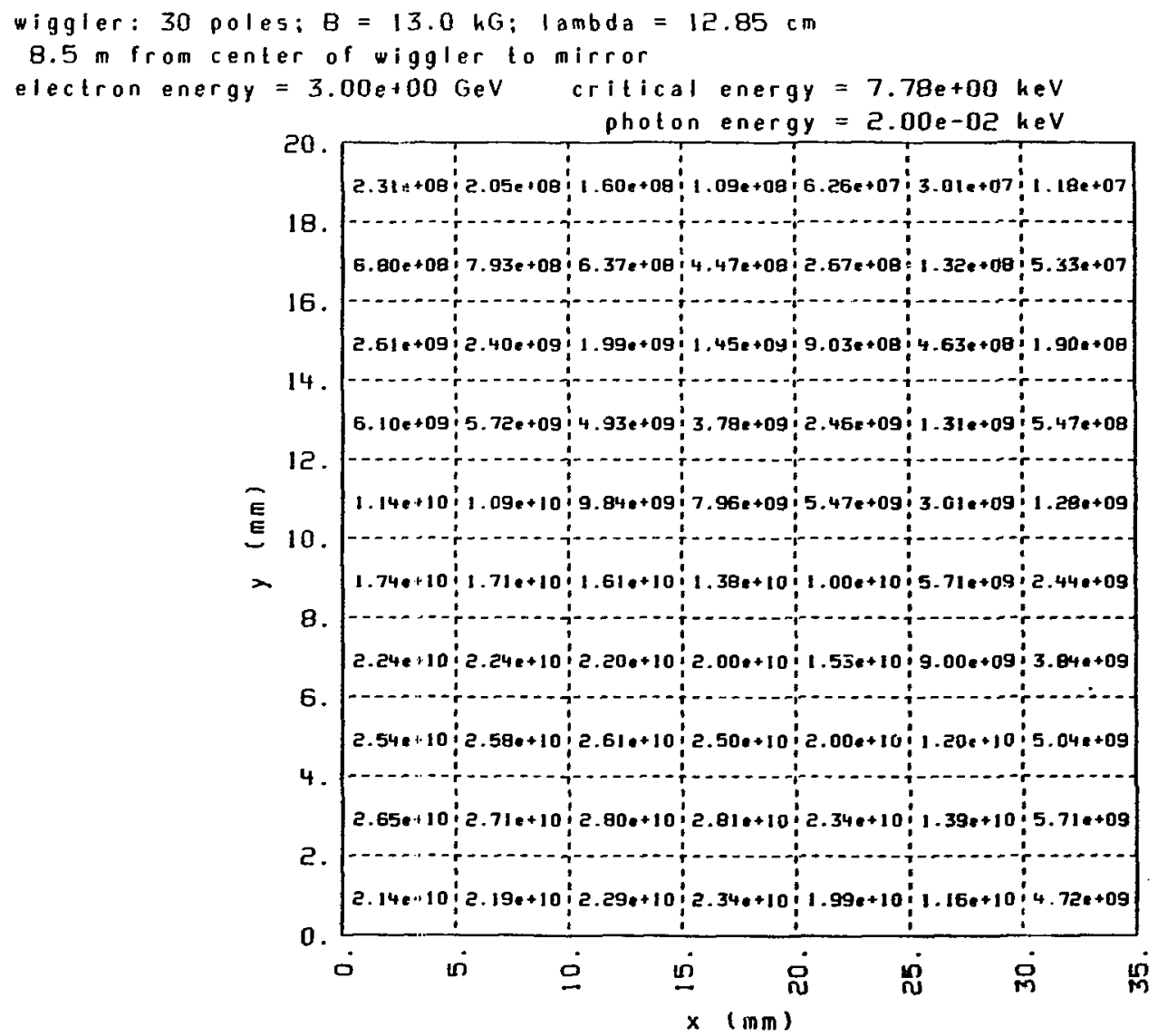

paralle I

polarization

photans/5ec/mA in $0.1 \%$ bandwidth 
Table C5.2.

wiggler: 30 poles; $B=13.0 \mathrm{kG} ; 1 \mathrm{ambda}=12.85 \mathrm{~cm}$ 8.5 from center of wiggler to mirror

electron energy $=3.00 \mathrm{e}+00 \mathrm{GeV}$ critical energy $=7.78 \mathrm{~T}+00 \mathrm{keV}$

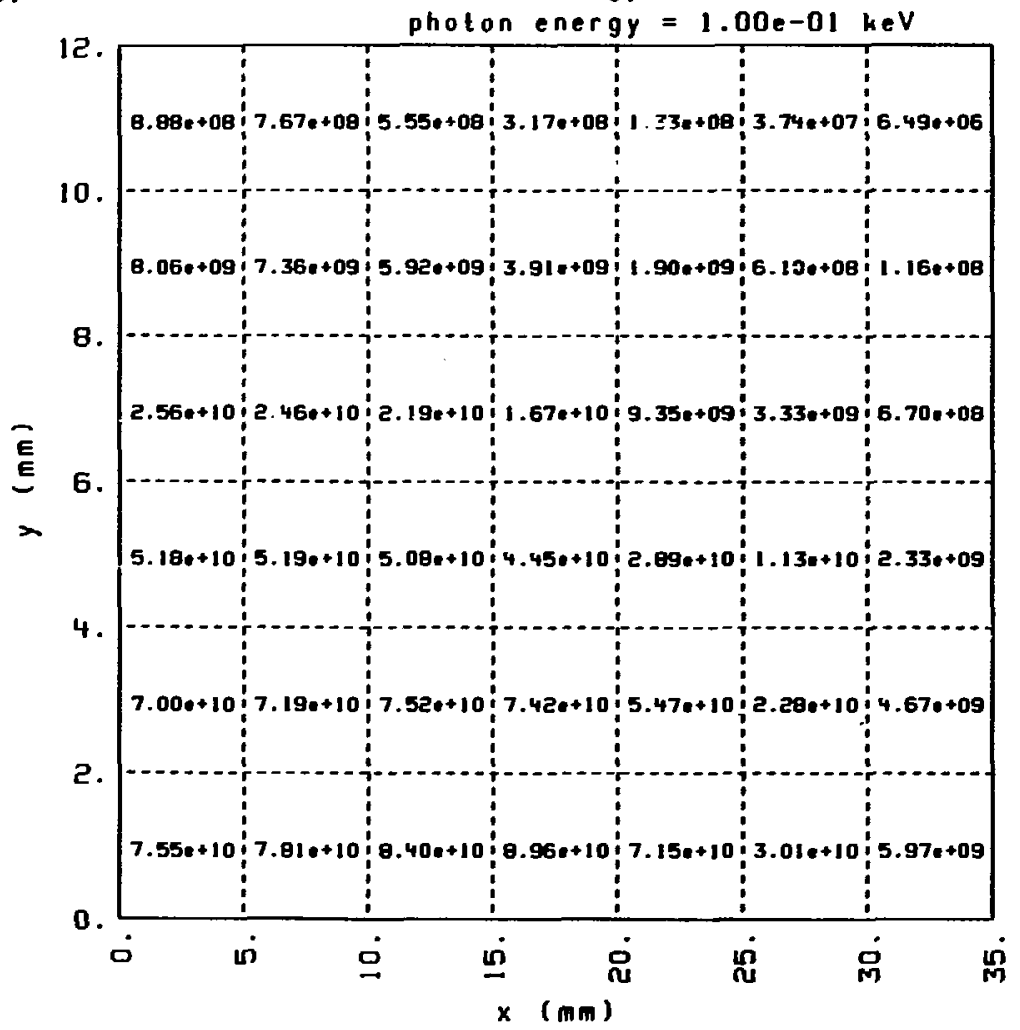

paral I el

polarization

photons/sec/mA In $0.1 \%$ bandwidth 
Table C5.3.

wiggler: 30 poles; $B=13.0 \mathrm{hG} ; 1 \mathrm{ambda}=12.85 \mathrm{~cm}$

$8.5 \mathrm{~m}$ from center of wiggler to mirror

electron energy $=3.00 \mathrm{e}+00 \mathrm{GeV}$ critical energy $=7.78 \mathrm{e}+00 \mathrm{heV}$

photon energy $=5.00 e-01$ keV

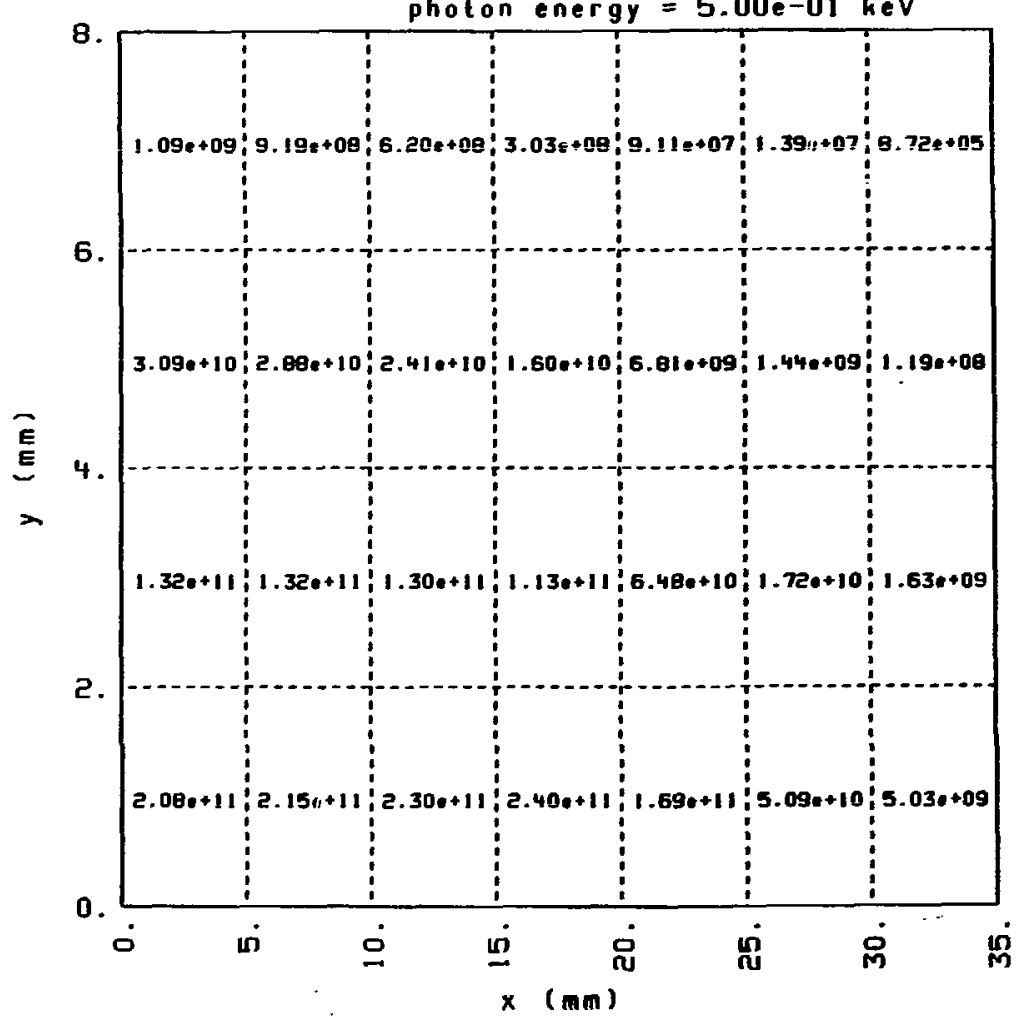

paral lel

polarization

photons/sec/mA in 0.17 bandwidth 
Table C5.4.

wiggler: 30 poles; $B=13.0 \mathrm{hG} ; 1$ ambda $=12.85 \mathrm{~cm}$ $8.5 \mathrm{~m}$ from center of wiggler to mirror electron energy $=3.00 \mathrm{e}+00 \mathrm{GeV}$ critical energy $=7.78 \mathrm{ta0} \mathrm{heV}$

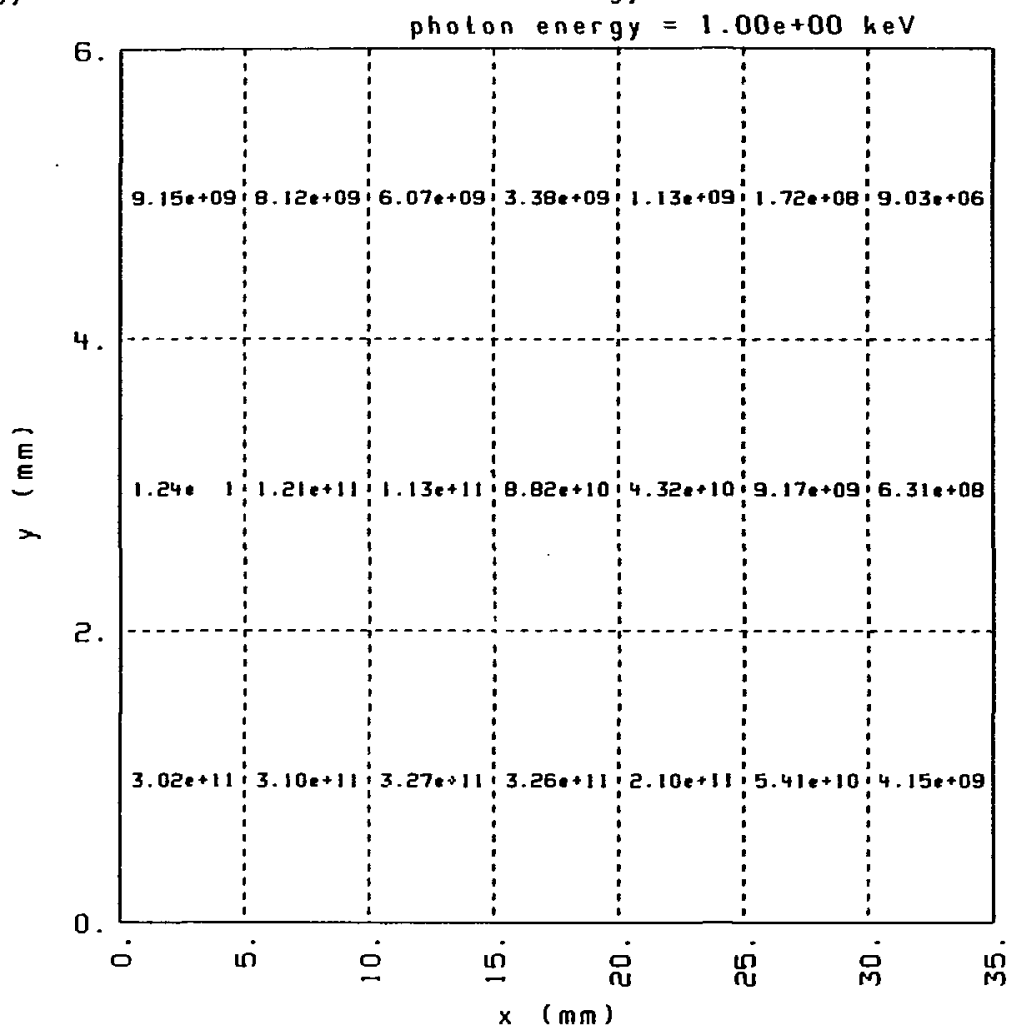

parallel

polarization 
Table C5.5.

wiggler: 30 poles; $B=13.0 \mathrm{kG} ; 1 \mathrm{ambda}=12.85 \mathrm{~cm}$ 8.5 m from center of wiggler to mirror

electron energy $=3.00 \mathrm{e}+00 \mathrm{GeV}$ critical energy $=7.78 \mathrm{e}+00 \mathrm{keV}$ photon energy $=3.89 \mathrm{e}+00 \mathrm{keV}$

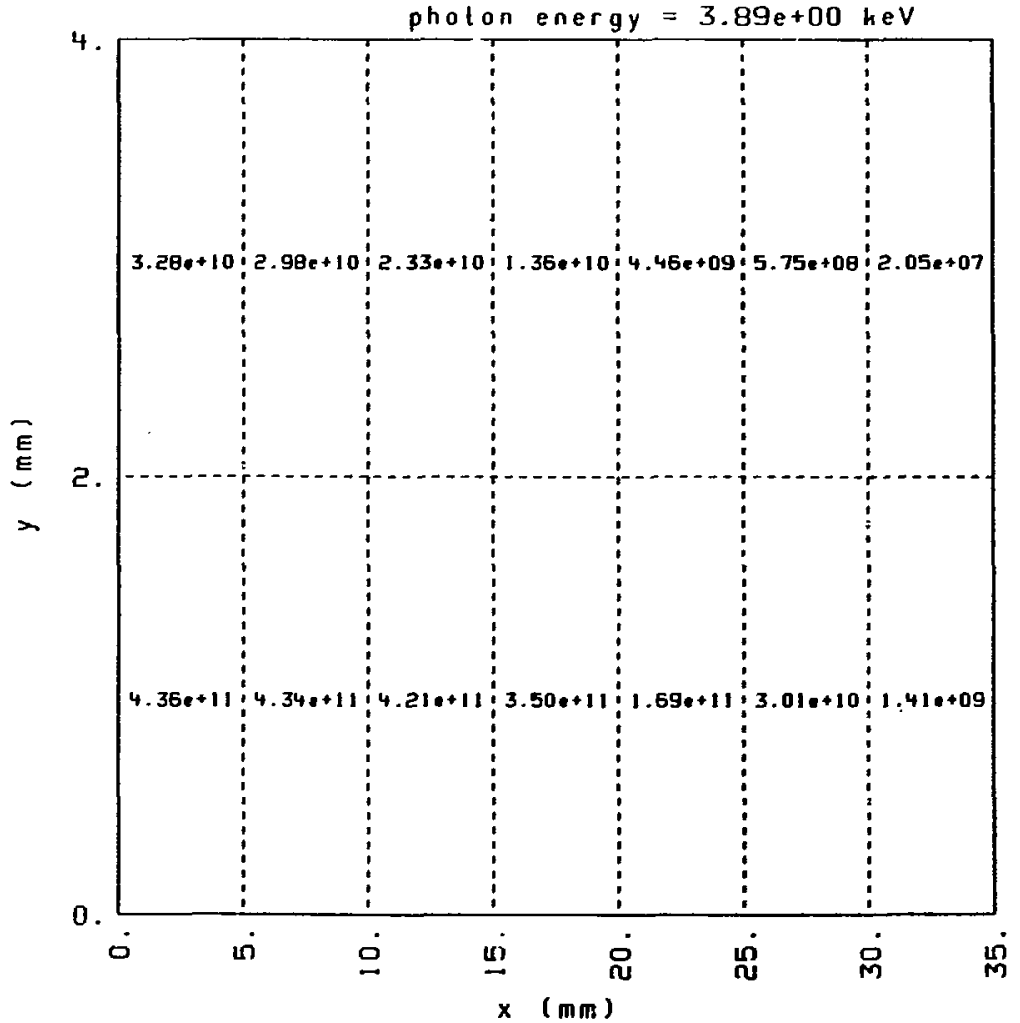

parallel

golarization

photons/sec/mA in $0.1 \%$ bandwidth 
Table C5.6.

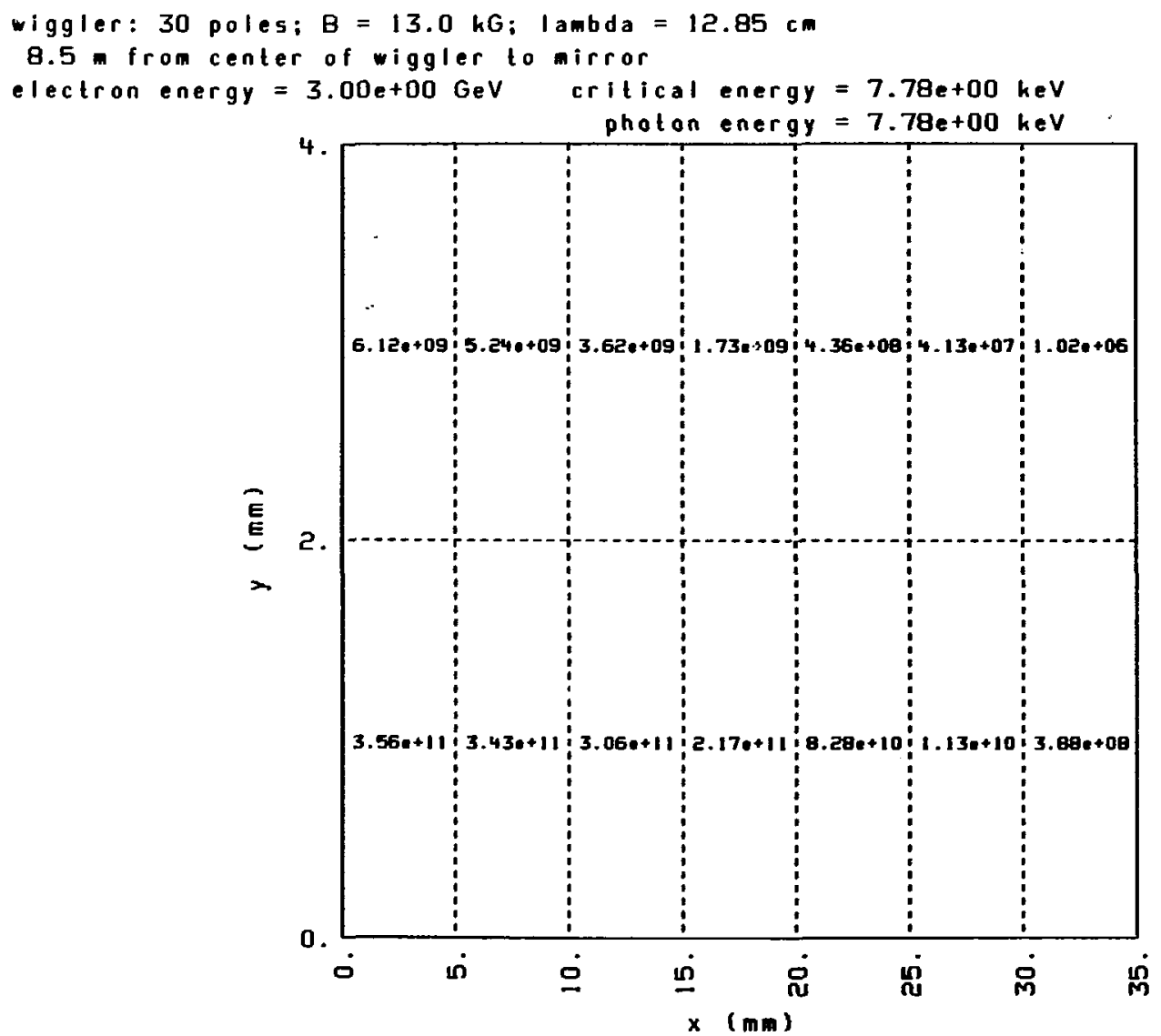

parallel

polarization

photons/sec/mA in 0.17 bandwidth 
Table C5.7.

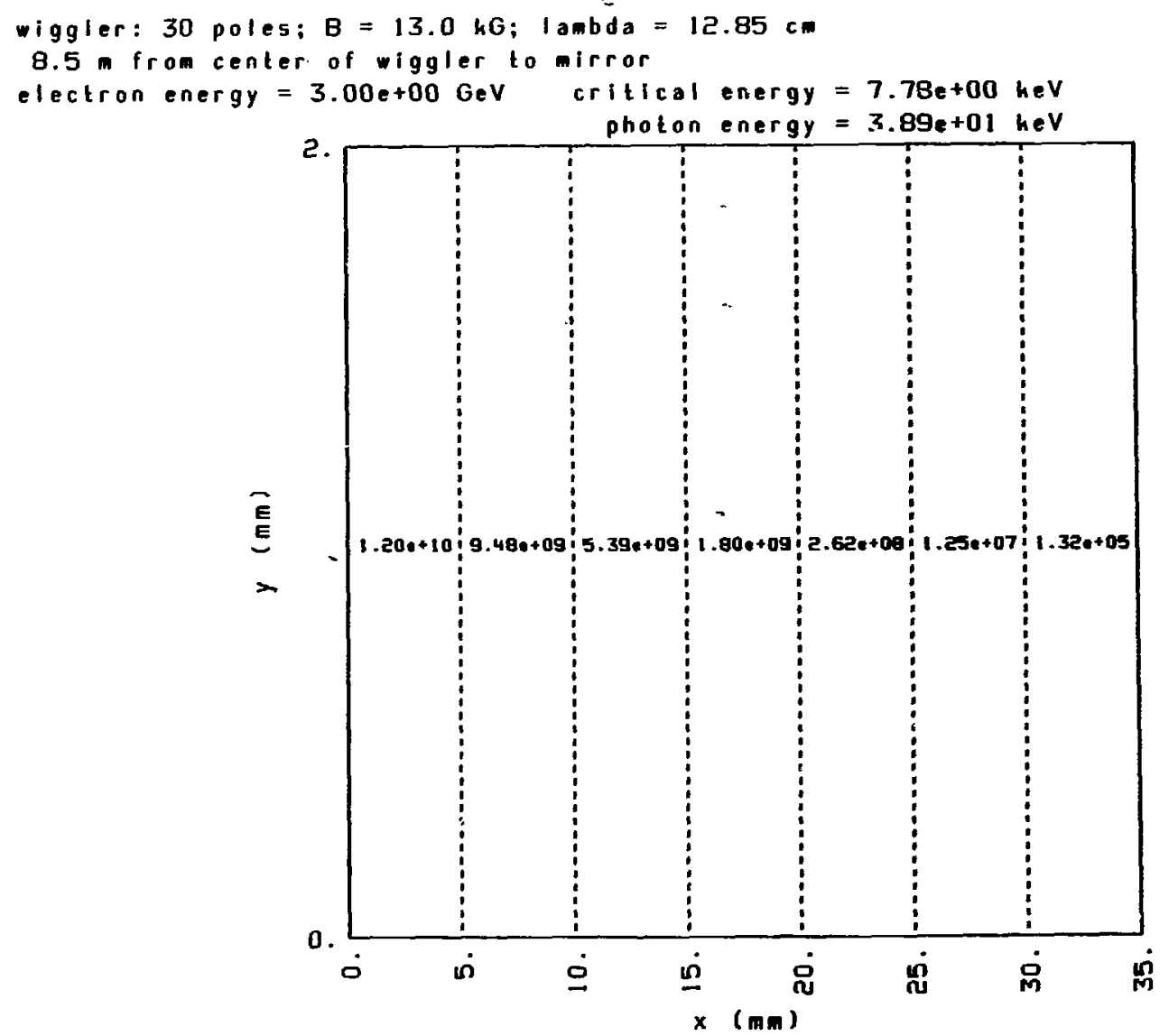

paral le l

polarization

photons/sec/mA in $0.1 \%$ bandwidth 
Table C6.1.

wiggler: 30 poles: $B=13.0 \mathrm{~kg} ; \mathrm{lambda}=12.85 \mathrm{~cm}$

$8.5 \mathrm{~m}$ from center of wiggler to mirror

electran energy $=3.00 \mathrm{e}+00 \mathrm{GeV}$ critical energy $=7.78 \mathrm{e}+00 \mathrm{keV}$

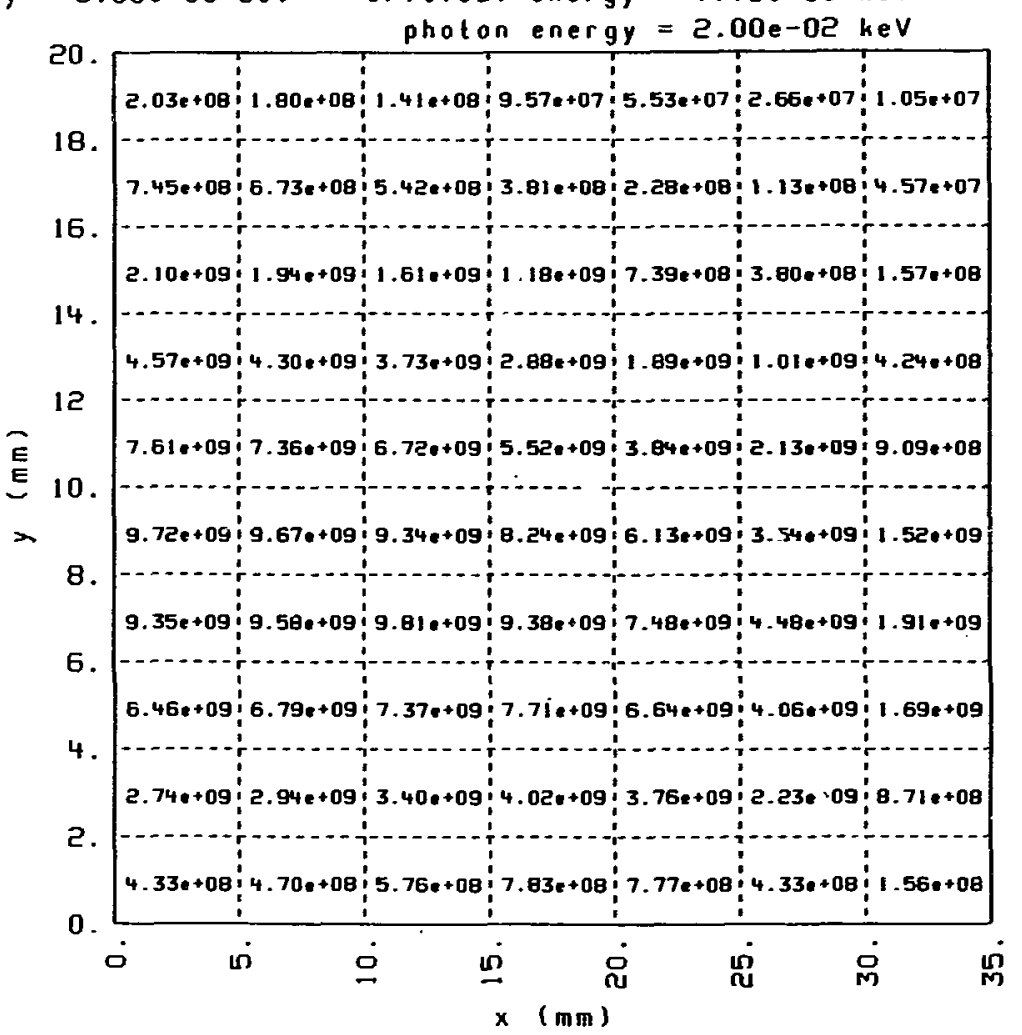

perpendicular

polarization

photons/sec/mA in $0.1 \%$ bandwidth 
Table C6.2.

wiggler: 30 poles; $B=13.0 \mathrm{hG} ; 1$ ambda $=12.85 \mathrm{~cm}$ 8.5 in from center of wiggler to mirrar

electron energy $=3.00 \mathrm{e}+00 \mathrm{GeV}$ critical energy $=7.78 \mathrm{e}+00 \mathrm{keV}$

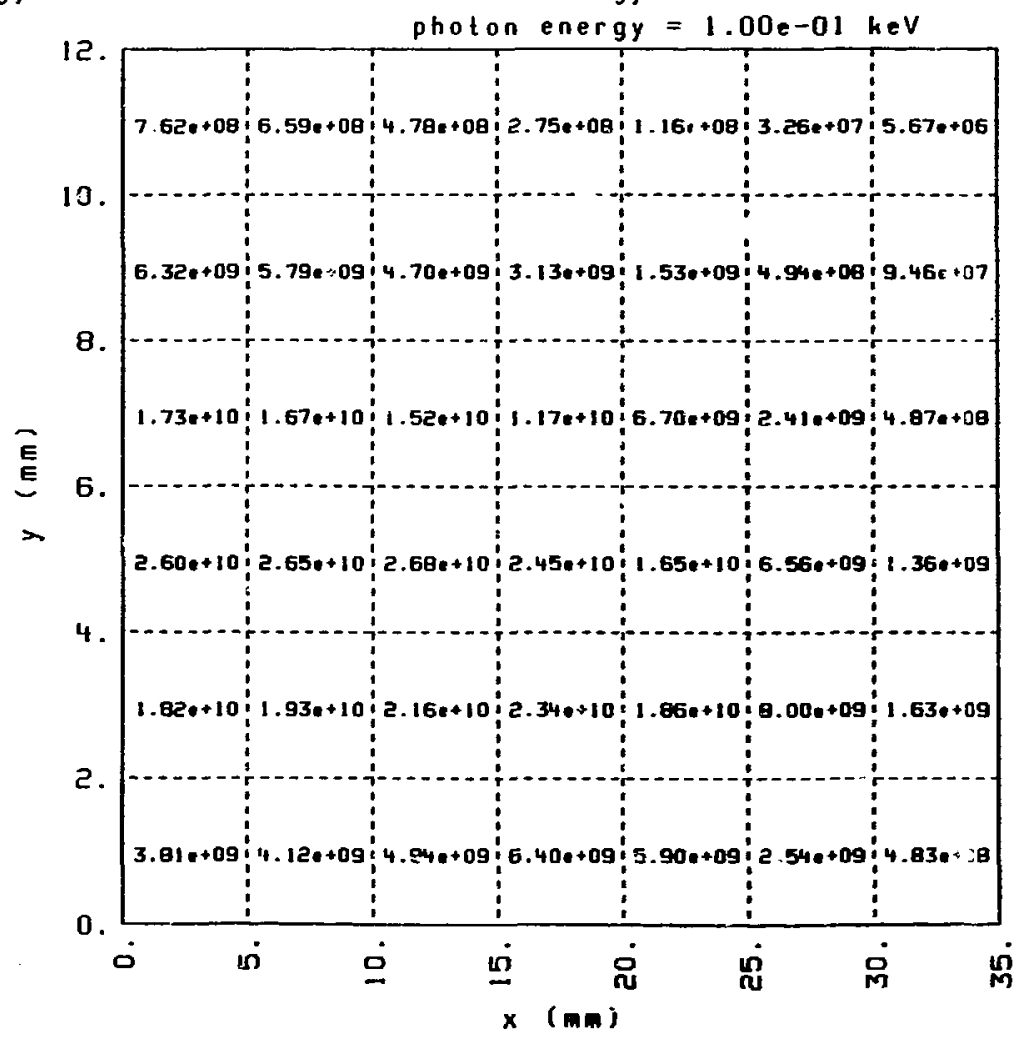

perpendicular polarization

photors/sec/mA in $0.1 \%$ bandwidth 
Table C6.3.

wiggler: 30 poles; $B=13.0 \mathrm{hG} ; \mathrm{Iambda}=12.85 \mathrm{~cm}$

8.5 m irom center of wiggter to mirror

electron energy $=3.00$ t00 GeV critical energy $=7.78$ tod heV photon energy $=5.00 \mathrm{e}-01 \mathrm{keV}$

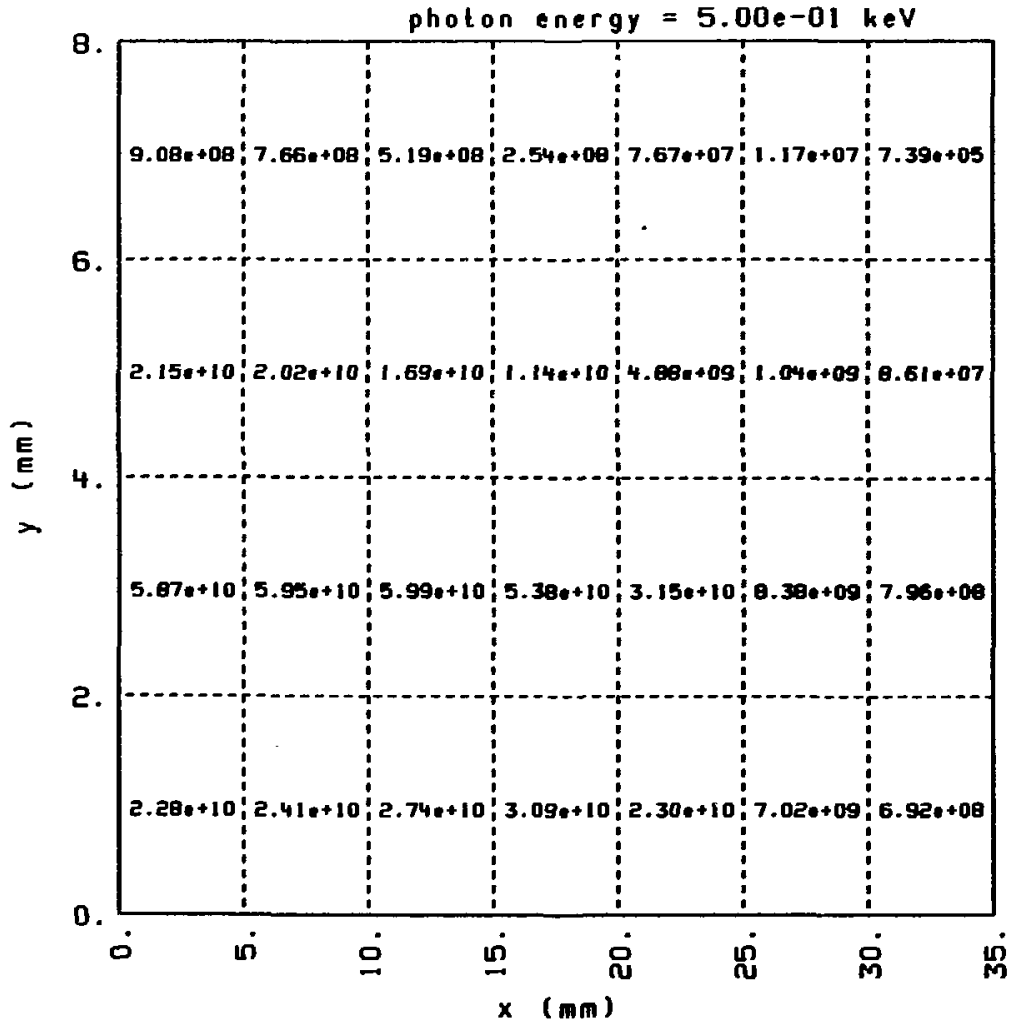

perpendicular polartzation 
Táble C6.4.

wiggler: 30 poles; $B=13.0 \mathrm{kG} ; 1 \mathrm{ambda}=12.85 \mathrm{~cm}$

$8.5 \mathrm{~m}$ from center of wiggler to mirror

electron energy $=3.00 \mathrm{e}+00 \mathrm{GeV}$ critical energy $=7.78 \mathrm{e}+00 \mathrm{keV}$

photon energy $=1.00 e+00 \mathrm{keV}$

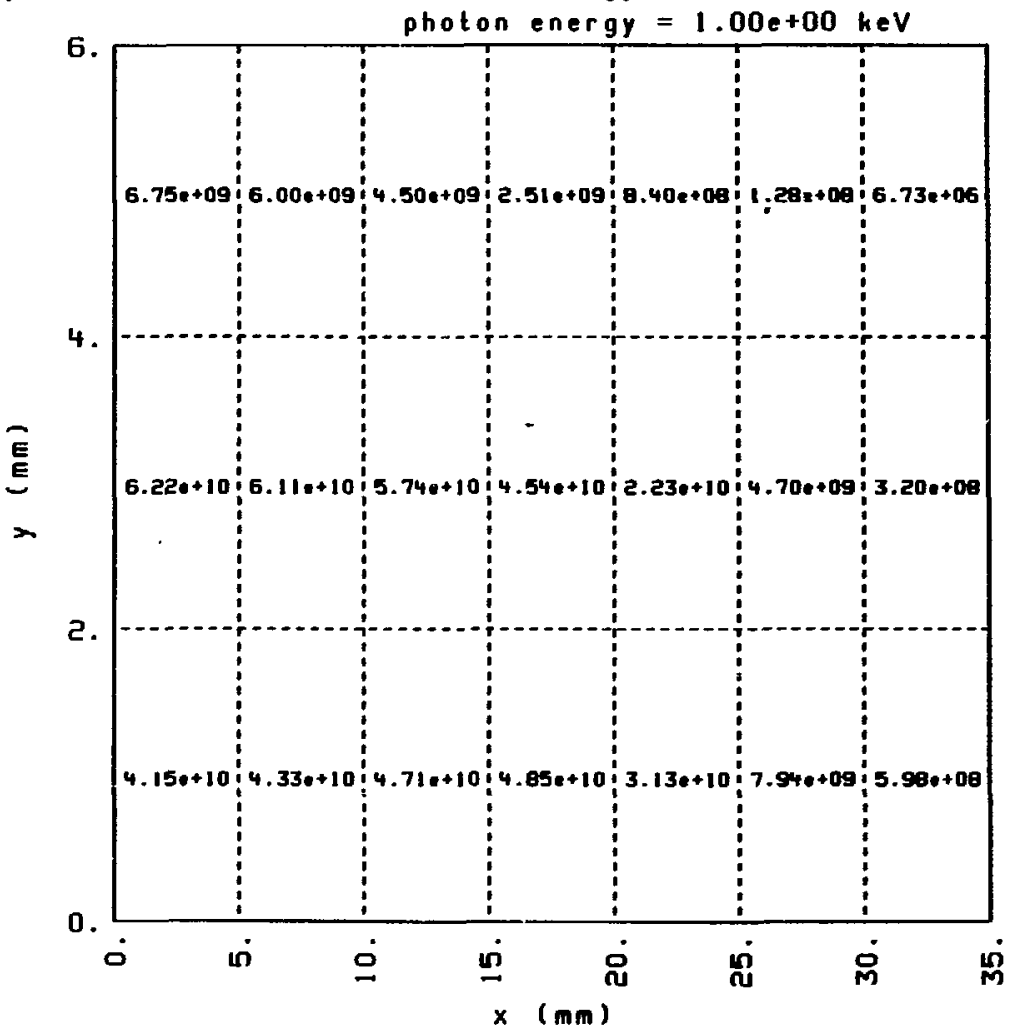

perpendicular

polarization

photons/sec/mA in 0.1X bandwidth 
Table C6.5.

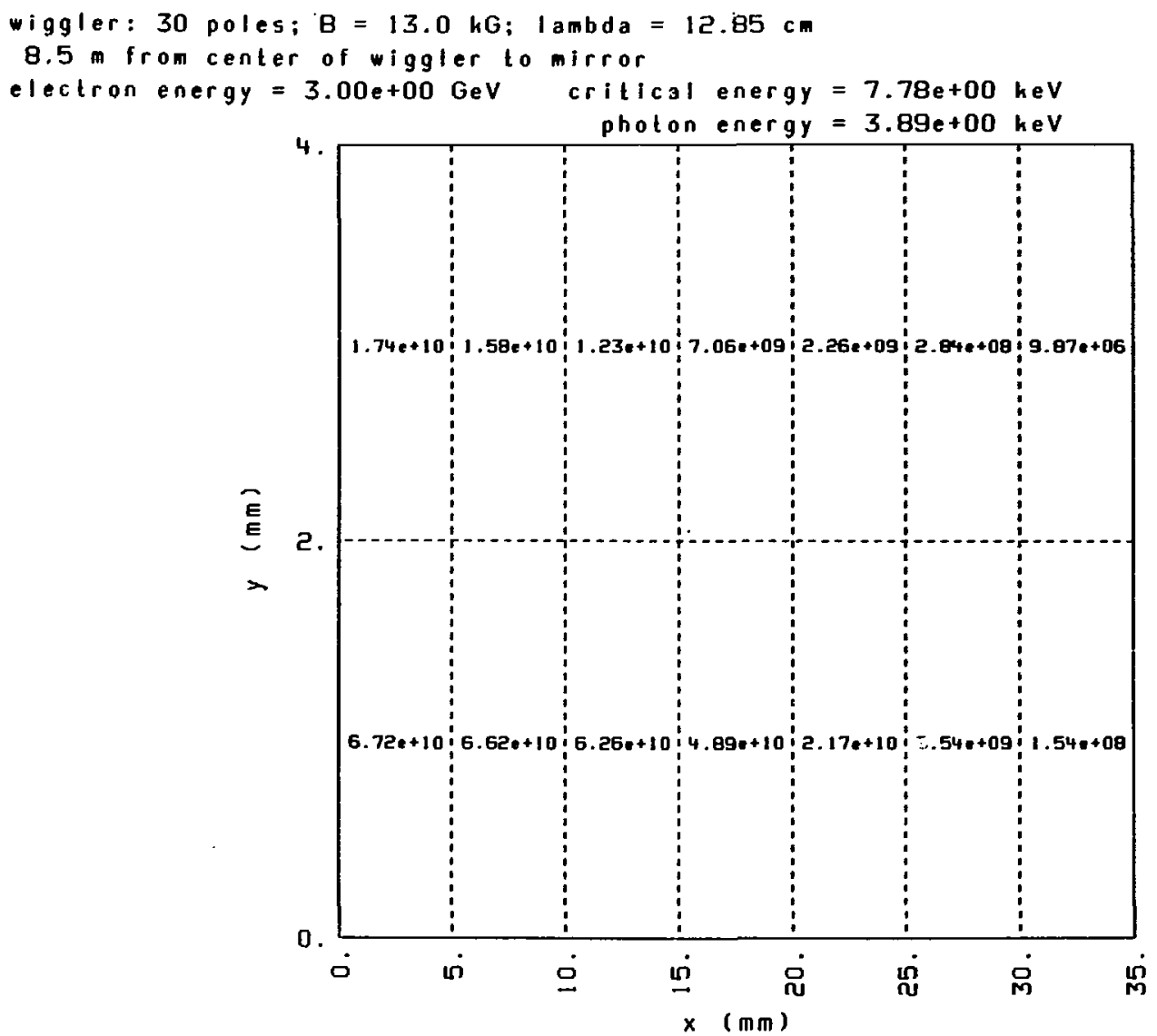

perpendicular

polarization

photons/sec/mA in $0.1 \%$ bandwidth 
Table C6.6.

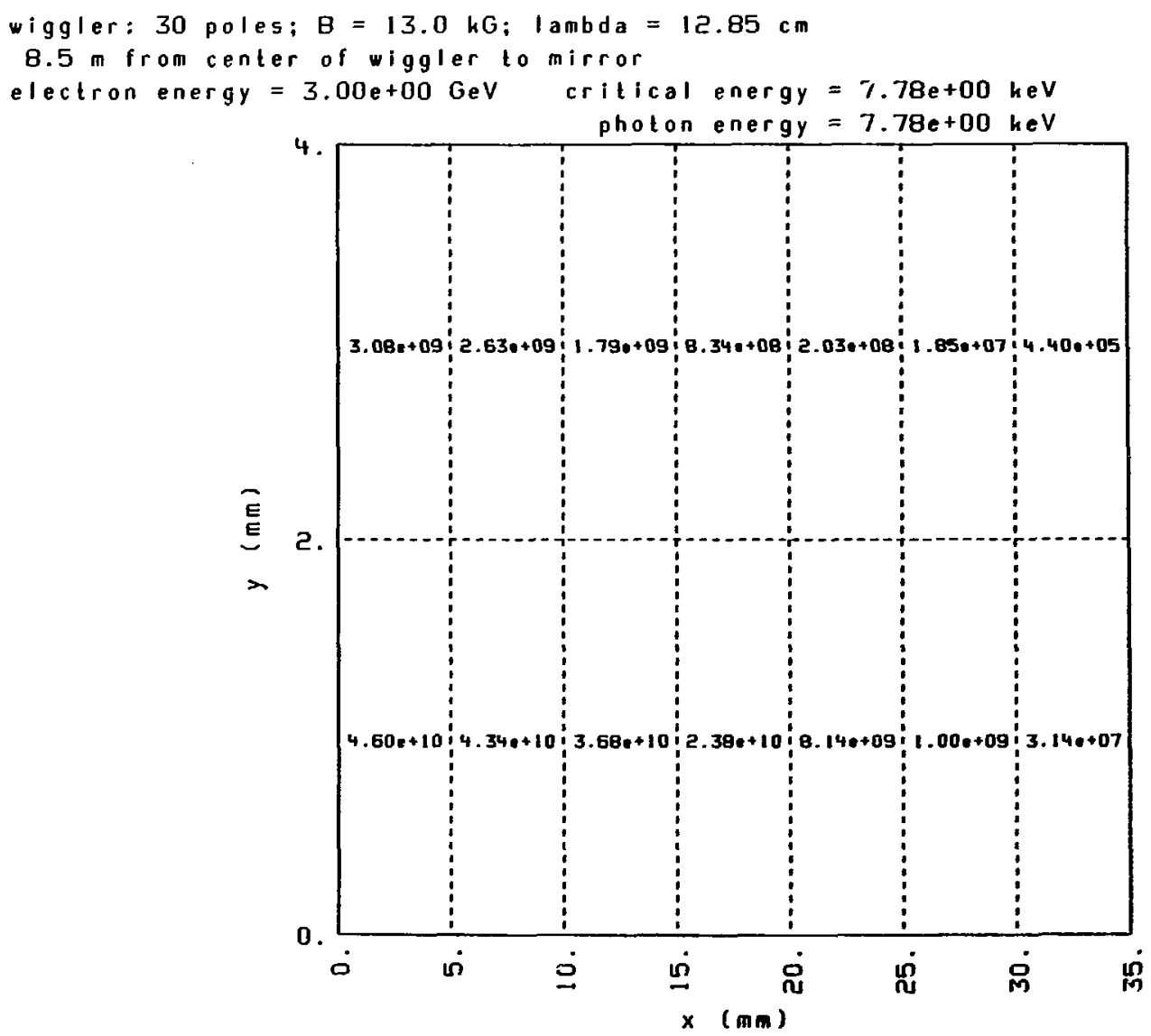

perpendicular

polarization

photons/sec/mA in 0.1X bandwidth 
Table C6.7.

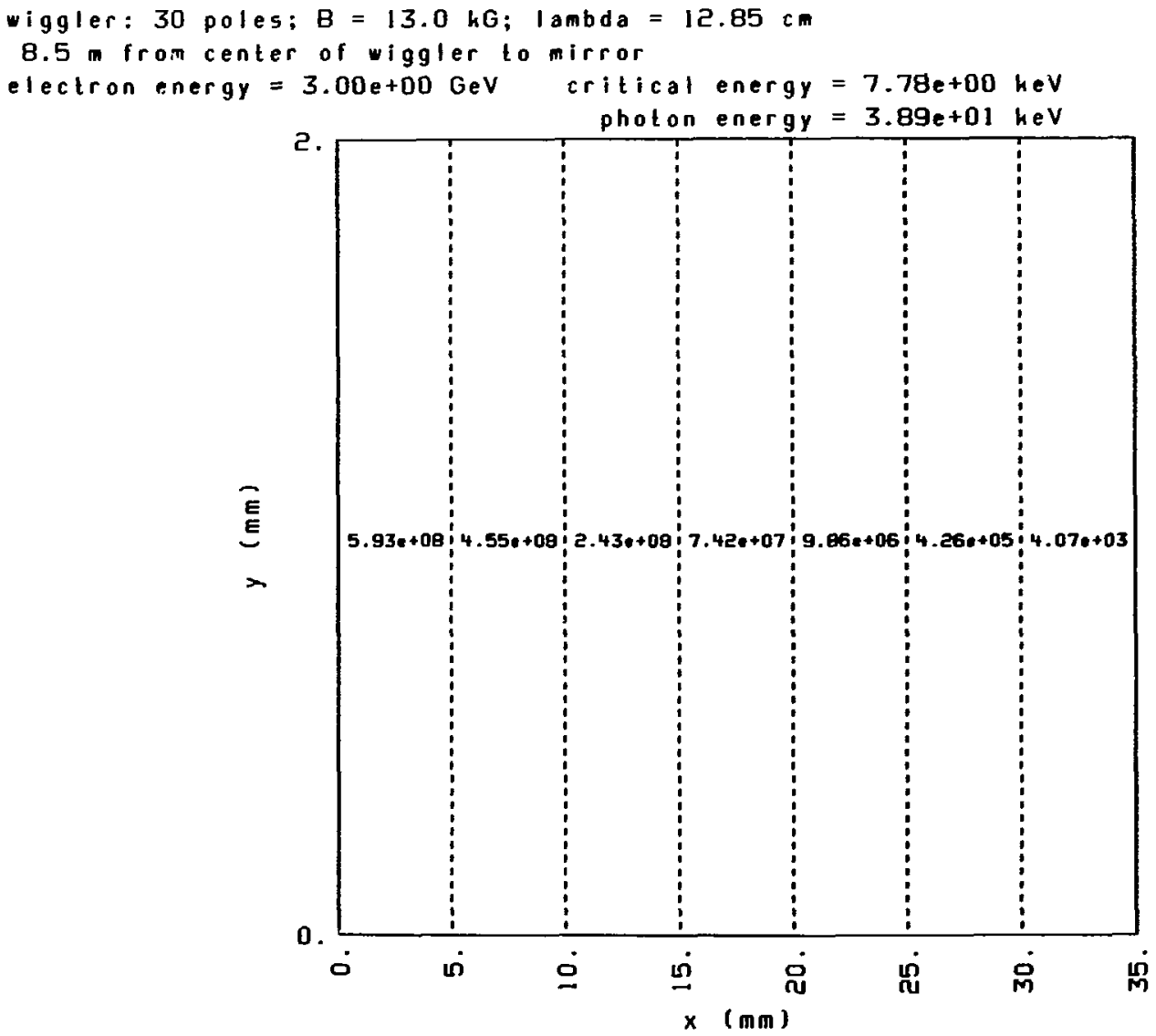

perpendicular polarization 
Table C7.1.

wiggler: 30 poles; $B=13.0 \mathrm{kG} ; \quad \operatorname{lambda}=12.85 \mathrm{~cm}$

$8.5 \mathrm{~m}$ from center of wiggler to mirror

electron energy $=3.40 \mathrm{e}+00 \mathrm{GeV}$ critical energy $=1.00 \mathrm{e}+01 \mathrm{heV}$

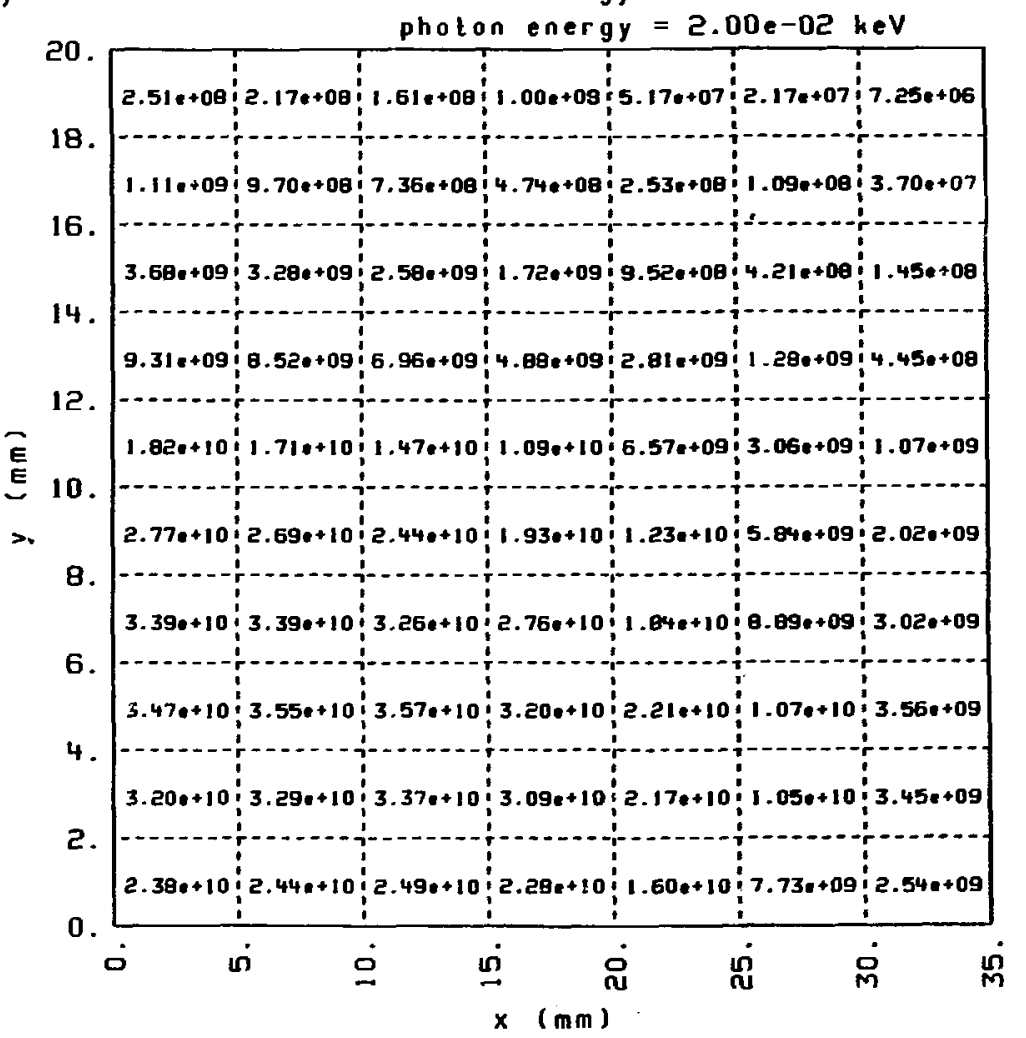

photons/sec/mA in $0.1 \%$ bandwidth 


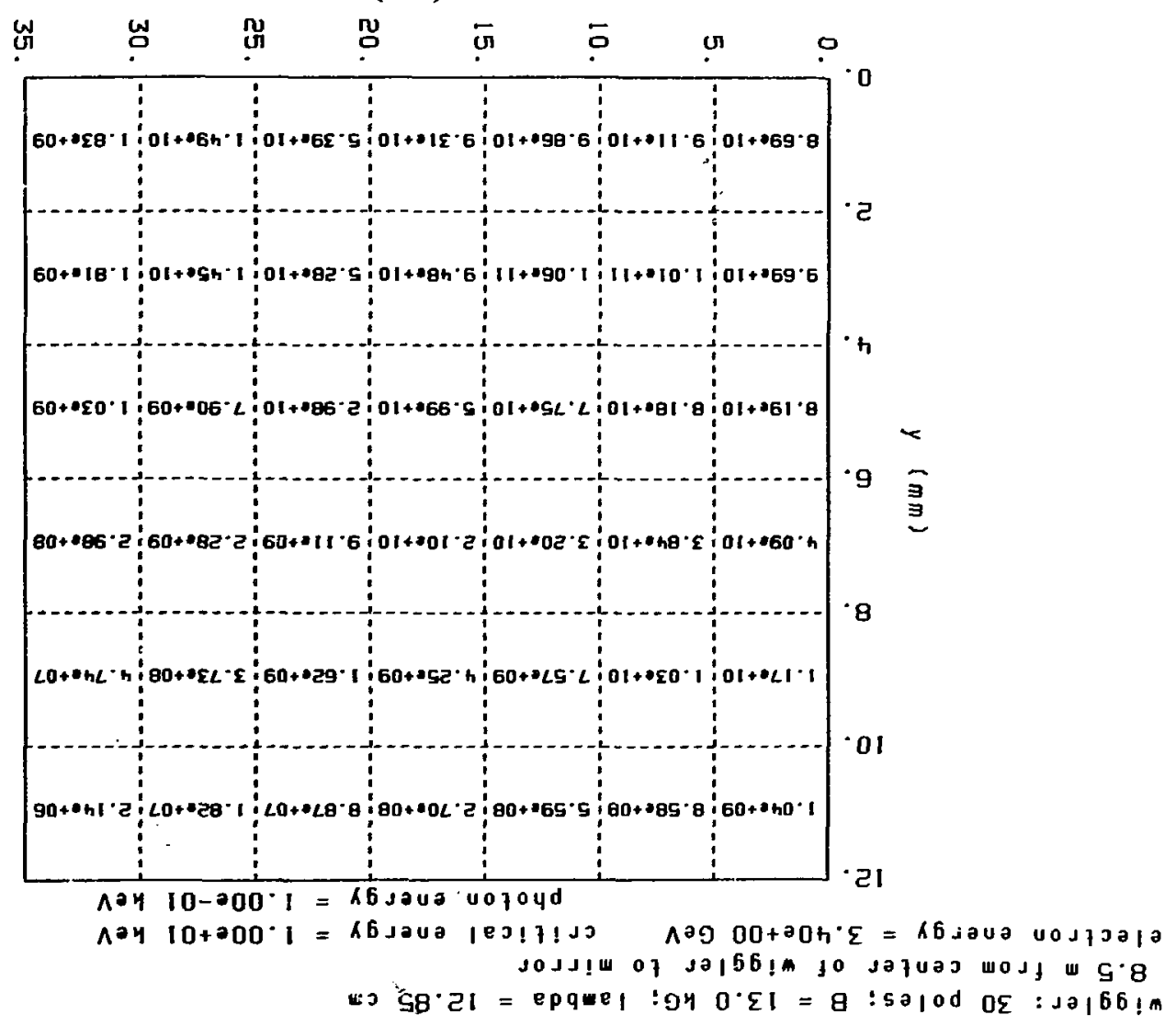


Table C7.3.

wiggler: 30 poles; $B=13.0 \mathrm{hG} ; 1$ ambda $=12.85 \mathrm{~cm}$

$8.5 \mathrm{~m}$ from center of wiggler to mirror

electron energy $=3.40 \mathrm{e}+00 \mathrm{GeV}$ critical energy $=1.00 \mathrm{ct}$ keV

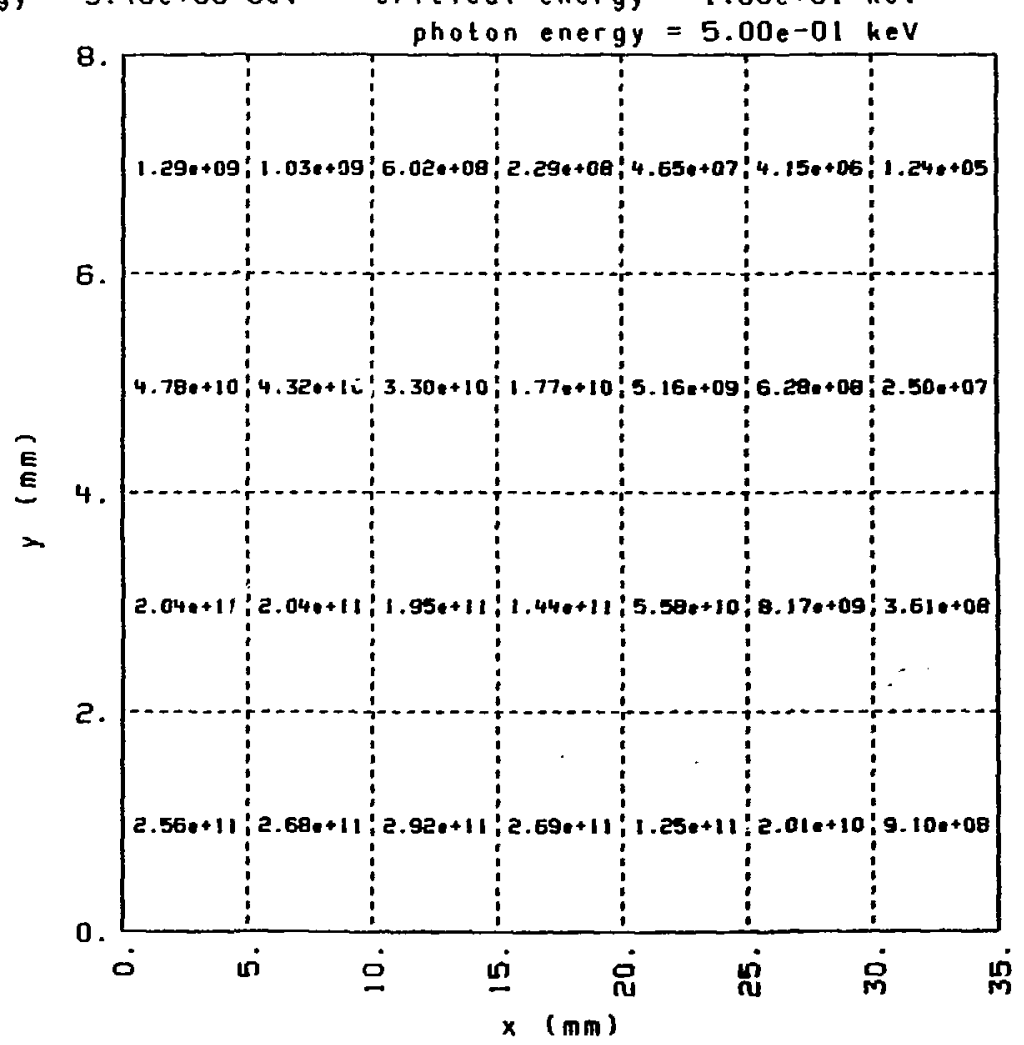

photons/sec/mA in $0.1 \%$ bandwidth 
Table C7.4.

wiggler: 30 poles; $B=13.0$ hG; lambda $=12.85 \mathrm{~cm}$ $8.5 \mathrm{~m}$ from center of wiggler to mirror

electron energy $=3.40 \mathrm{e}+00 \mathrm{GeV}$ critical energy $=1.00 \mathrm{e}+0 \mathrm{~h} \mathrm{heV}$ photon energy $=1.00 \mathrm{e}+00 \mathrm{keV}$

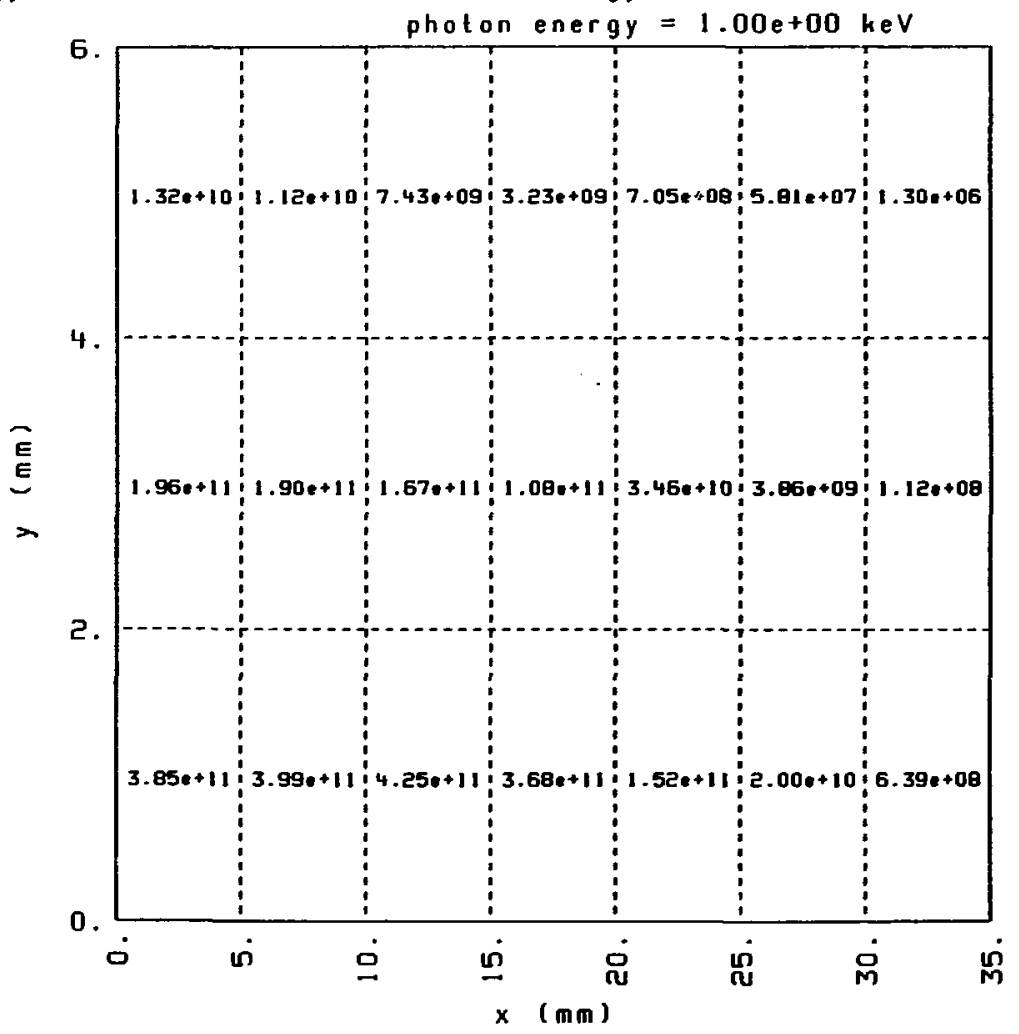

photons/sec/mA in 0.Iz bandwidth 
Table C7.5.

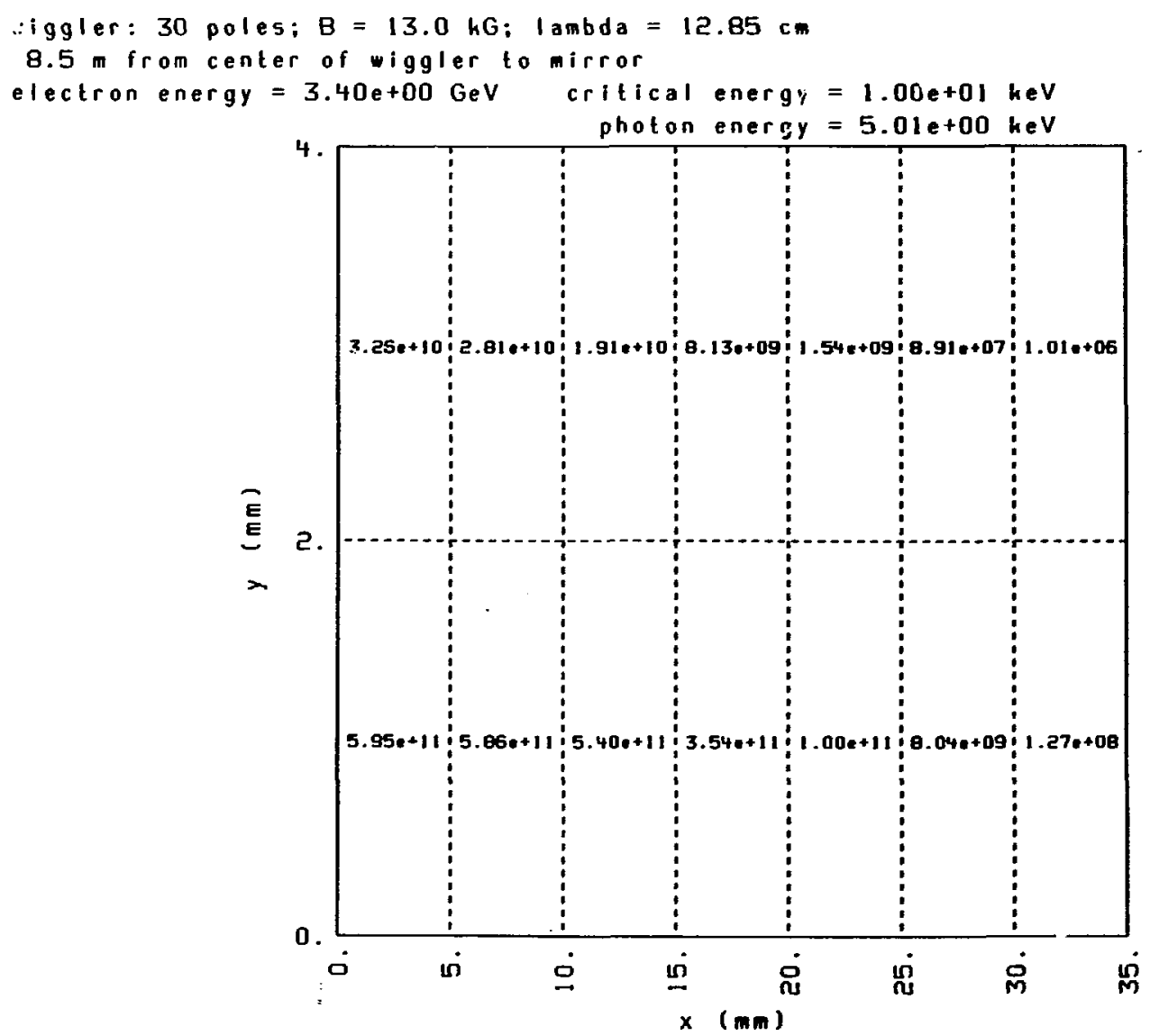

photons/sec/mA in 0.17 bandwidth 
Table C7.6.

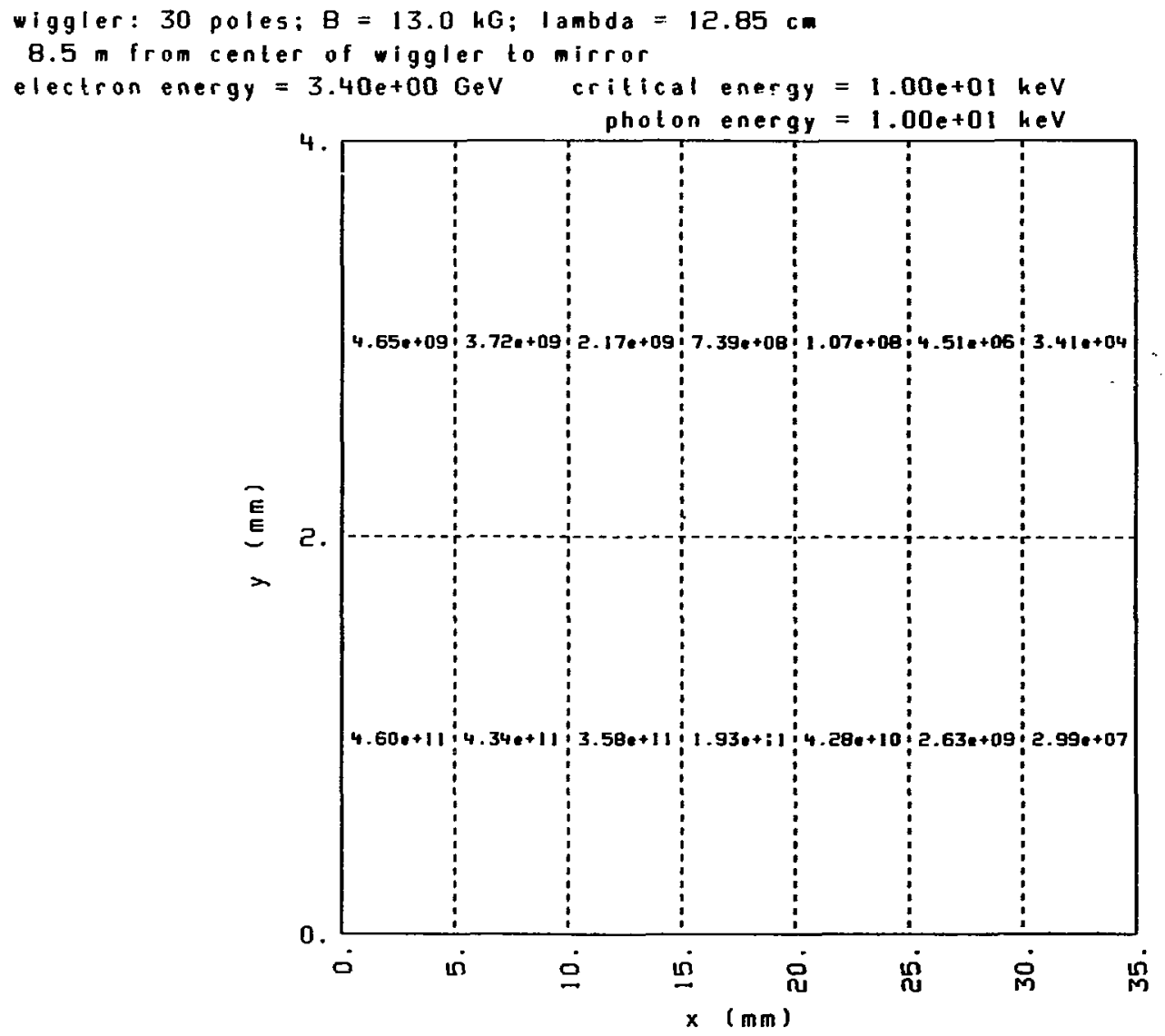

pholons/sec/mA in $0.1 \%$ bandwidth 
Table C7.7.

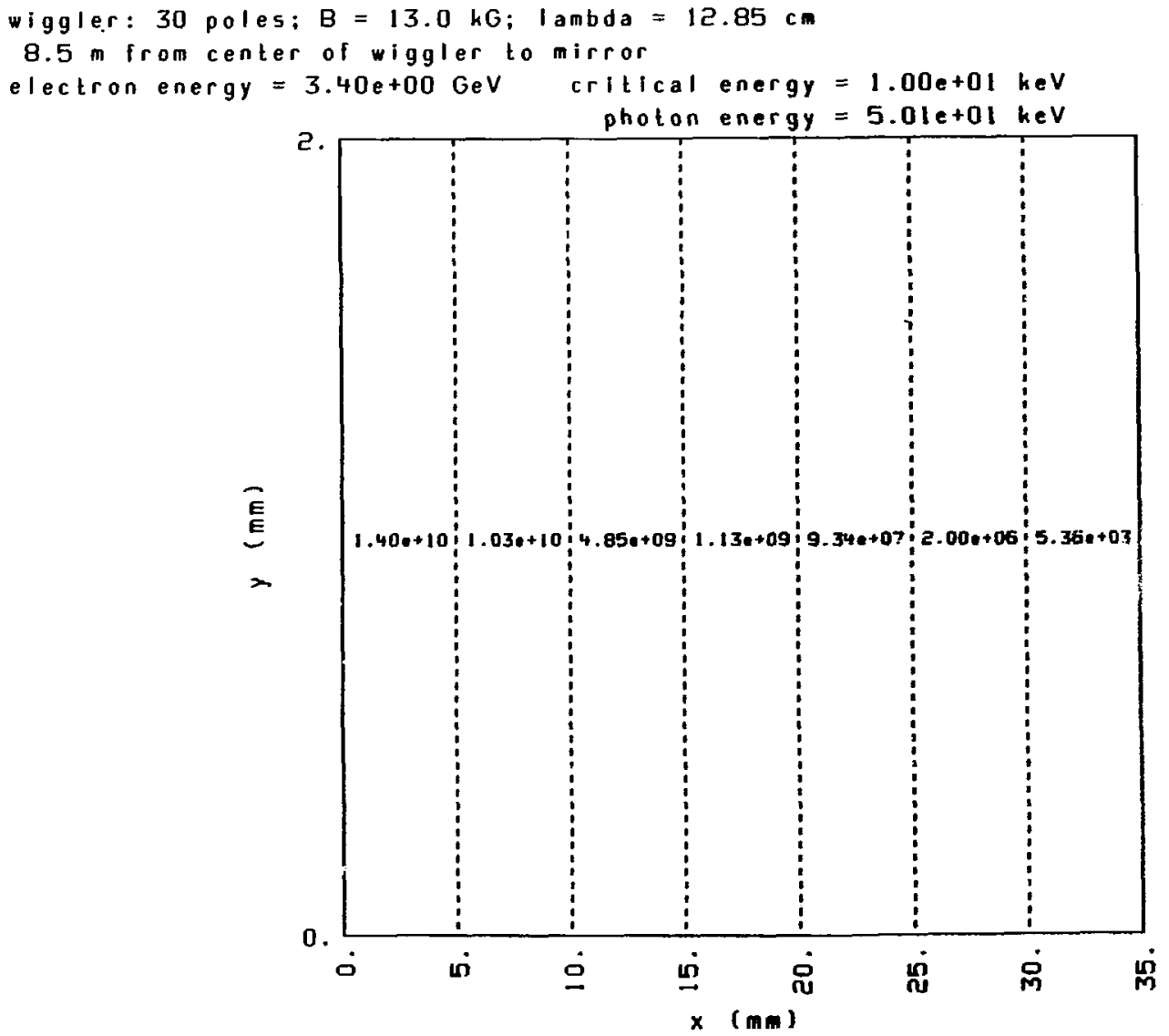

photons/sec/mA in $0.1 \%$ bandwidth 
Table C8.1.

wiggler: 30 poles; $B=13.0 \mathrm{hG}$ : I ambda $=12.85 \mathrm{~cm}$ Q.5 m from center of wiggler to mirror

electron energy $=3.40 \mathrm{e}+00 \mathrm{GeV}$ critical energy $=1.00 \mathrm{e}+01 \mathrm{heV}$

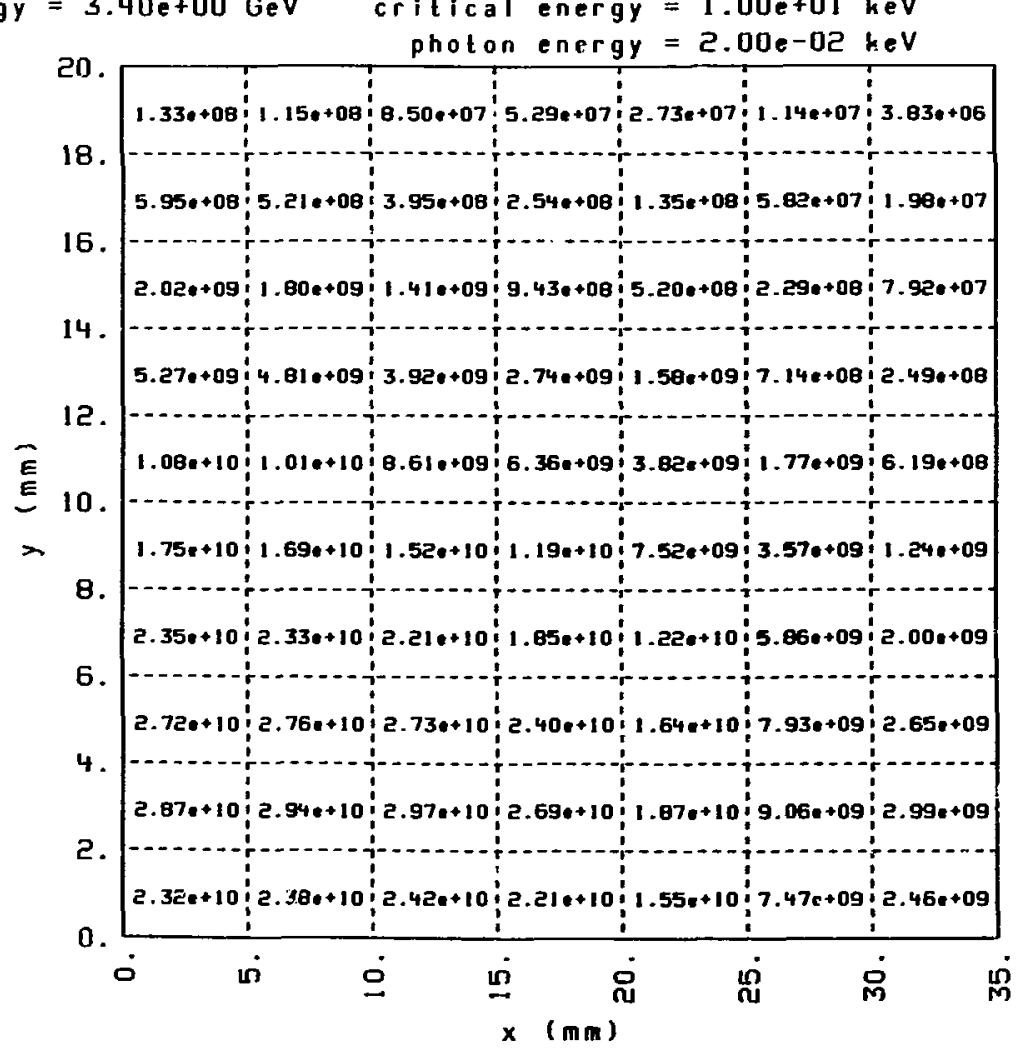

paral le I

polarization

photons/sec/mA in 0.17 bandwidth 
Table C8.2.

wiggler: 30 poles; $B=13.0 \mathrm{kG} ; \operatorname{lambda}=12.85 \mathrm{~cm}$

8.5 m from center of wiggler to mirror

electron energy $=3.40 e+00 \mathrm{GeV}$ critical energy $=1.00 \mathrm{e}+01 \mathrm{heV}$

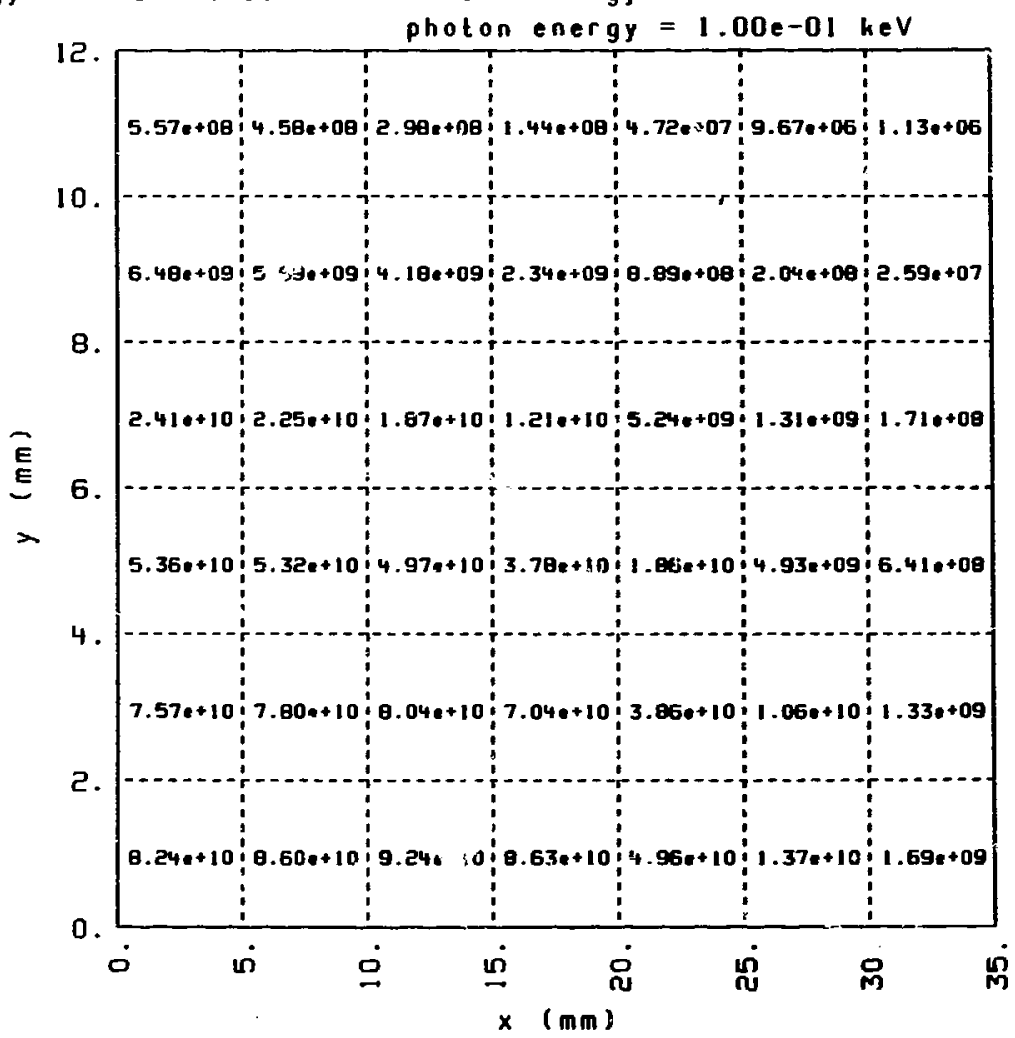

parallel

polarization

photons/sec/mA in $0.1 \%$ bandwidth 
Table C8.3.

wiggler: 30 pales; $B=13.0 \mathrm{hG;} 1$ ambda $=12.85 \mathrm{~cm}$ $8.5 \mathrm{~m}$ from center of wiggler to mirror

electron ent:gy $=3.40 \mathrm{e}+00 \mathrm{GeV}$

crilical energy $=1.00 e+0 l$ keV

photon energy $=5.00 e-01$ heV

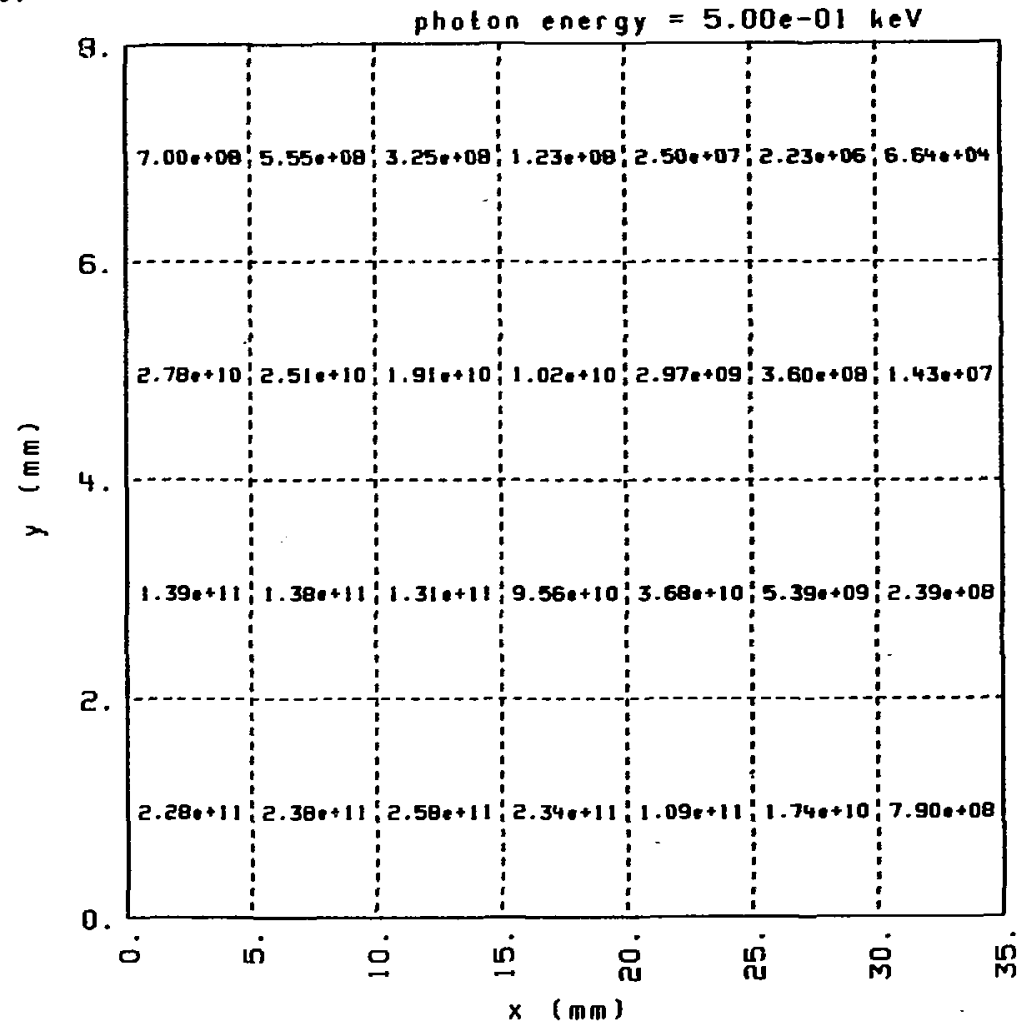

parallel

polarization

photons/sec/mA in $0.1 \%$ bandwidth 
Table C8.4.

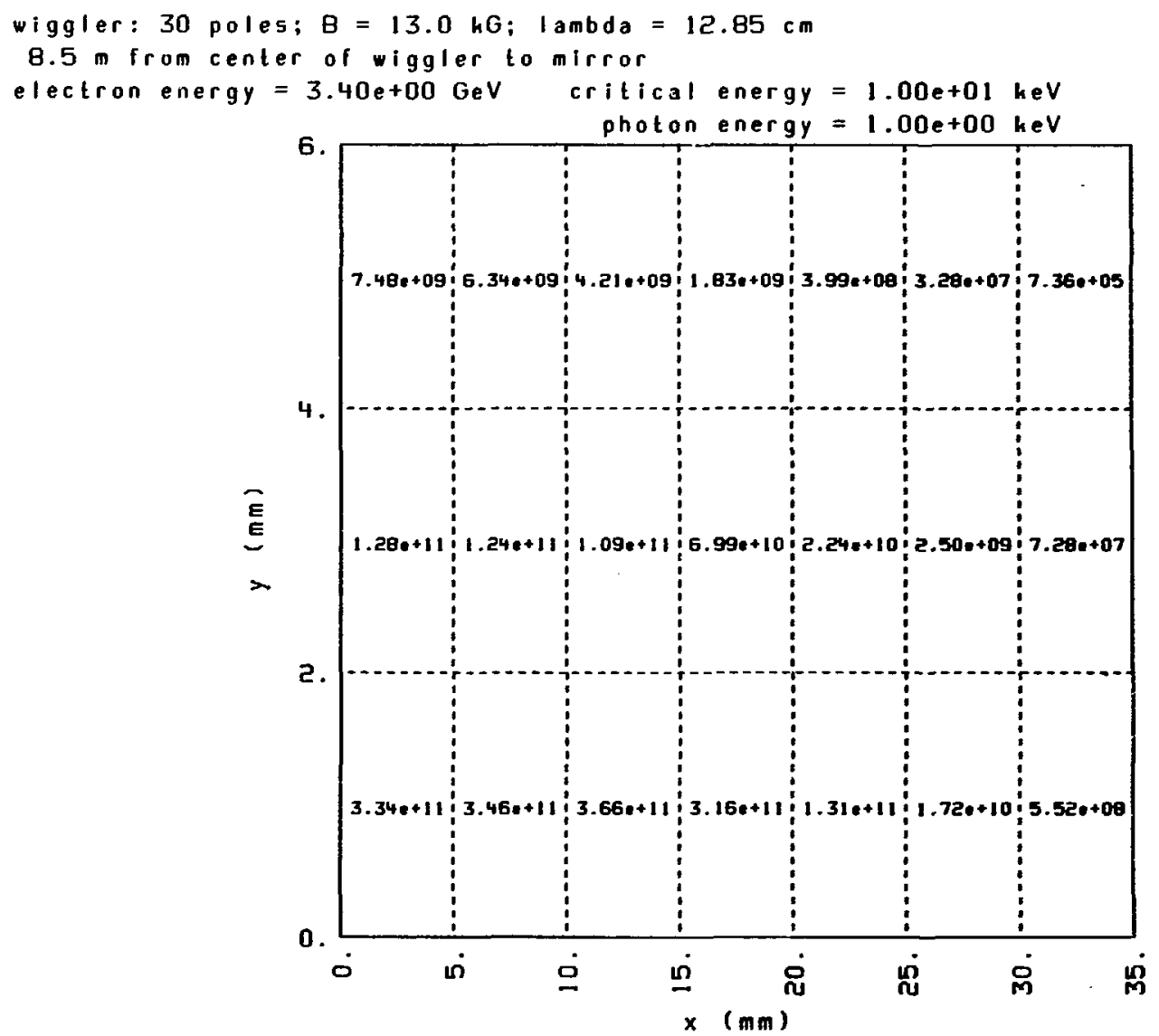

paral lel

potarization

photons/sec/mA in 0.1 t bandwidth 
Table C8.5.

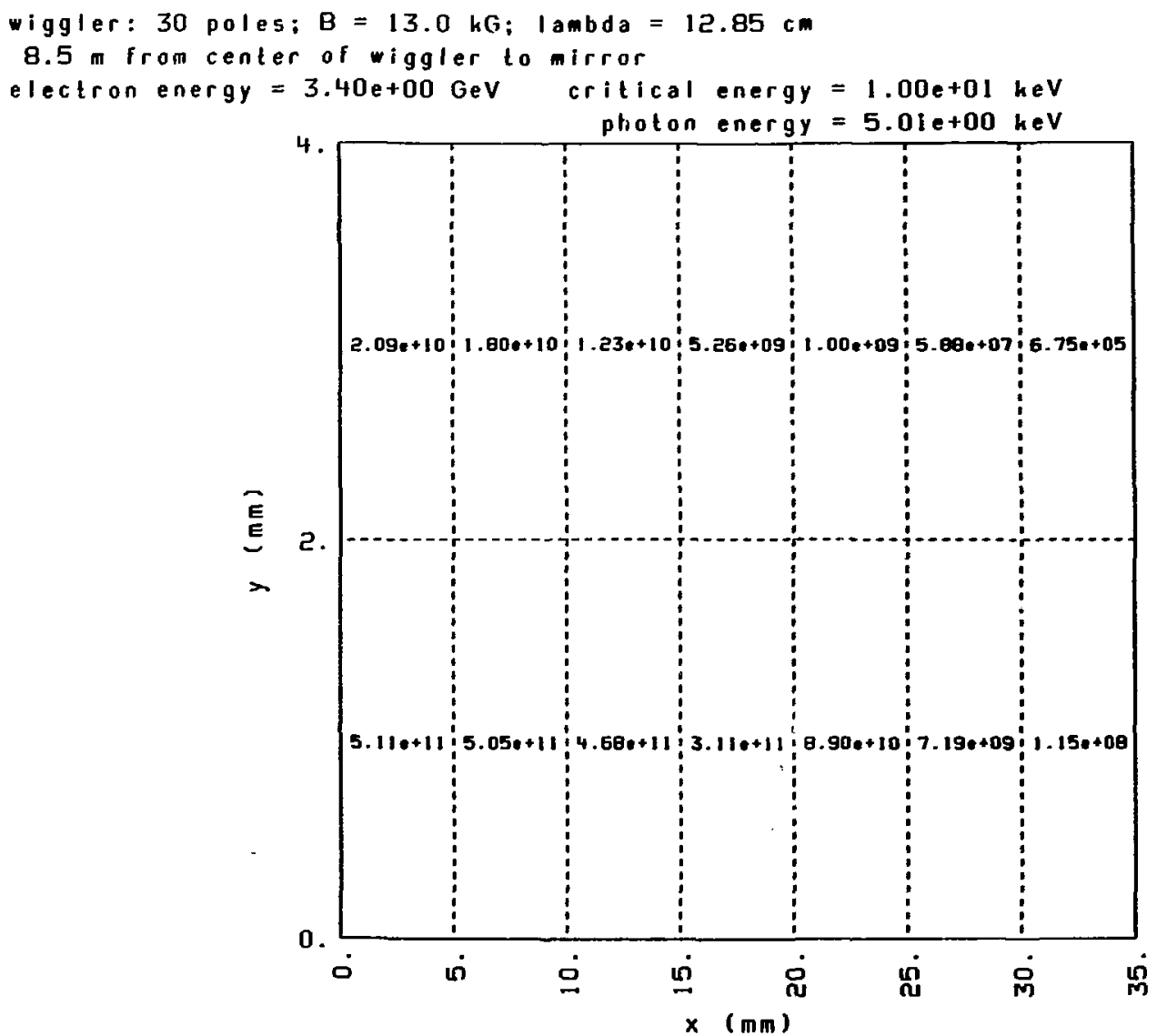

paral le l

polarization

photons/sec/mA in $0.1 x$ bandwidth 
Table C8.6.

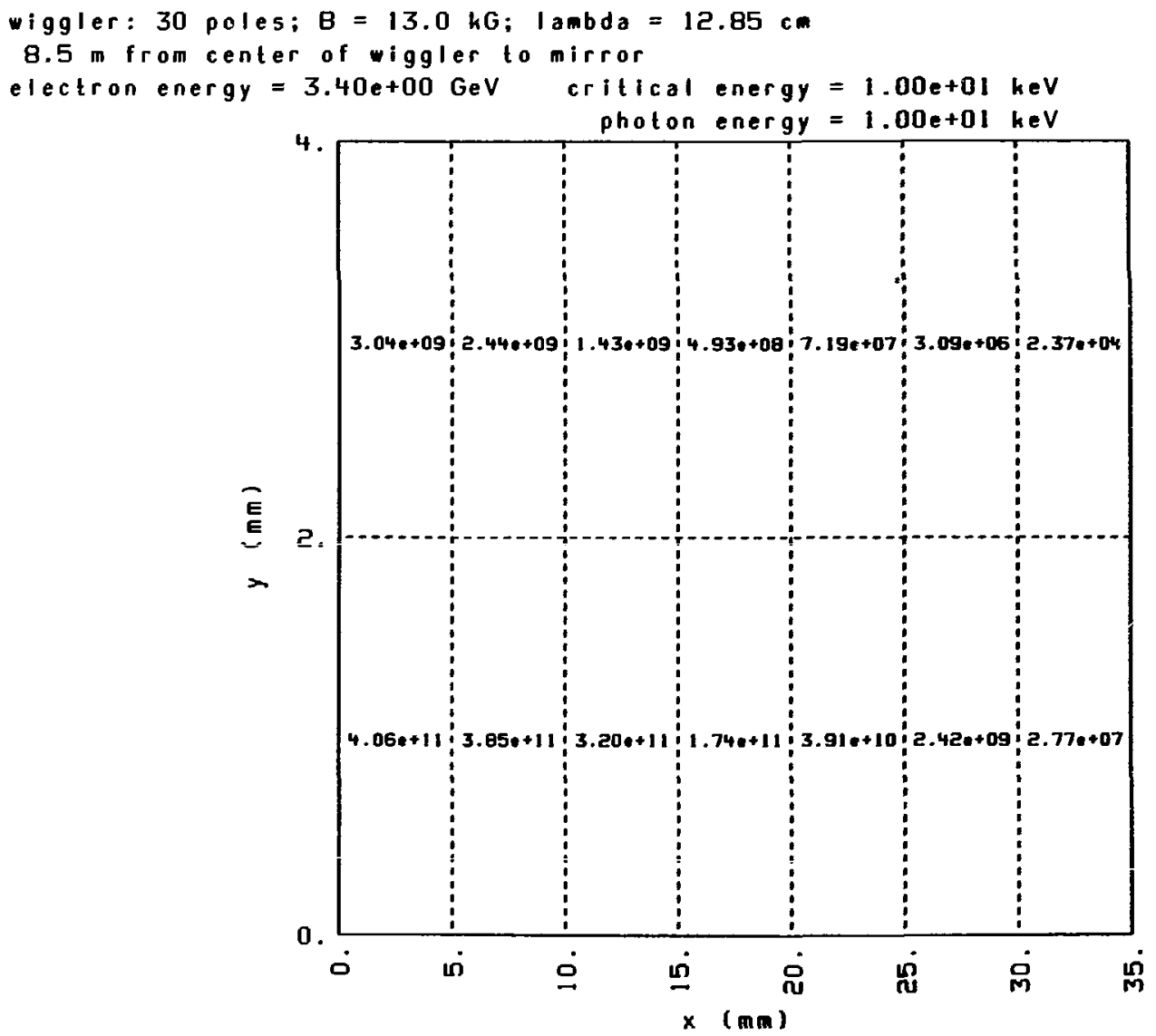

parallel

polarization

photons/sec/mA in 0.1X bandwidth 
Table C8.7.

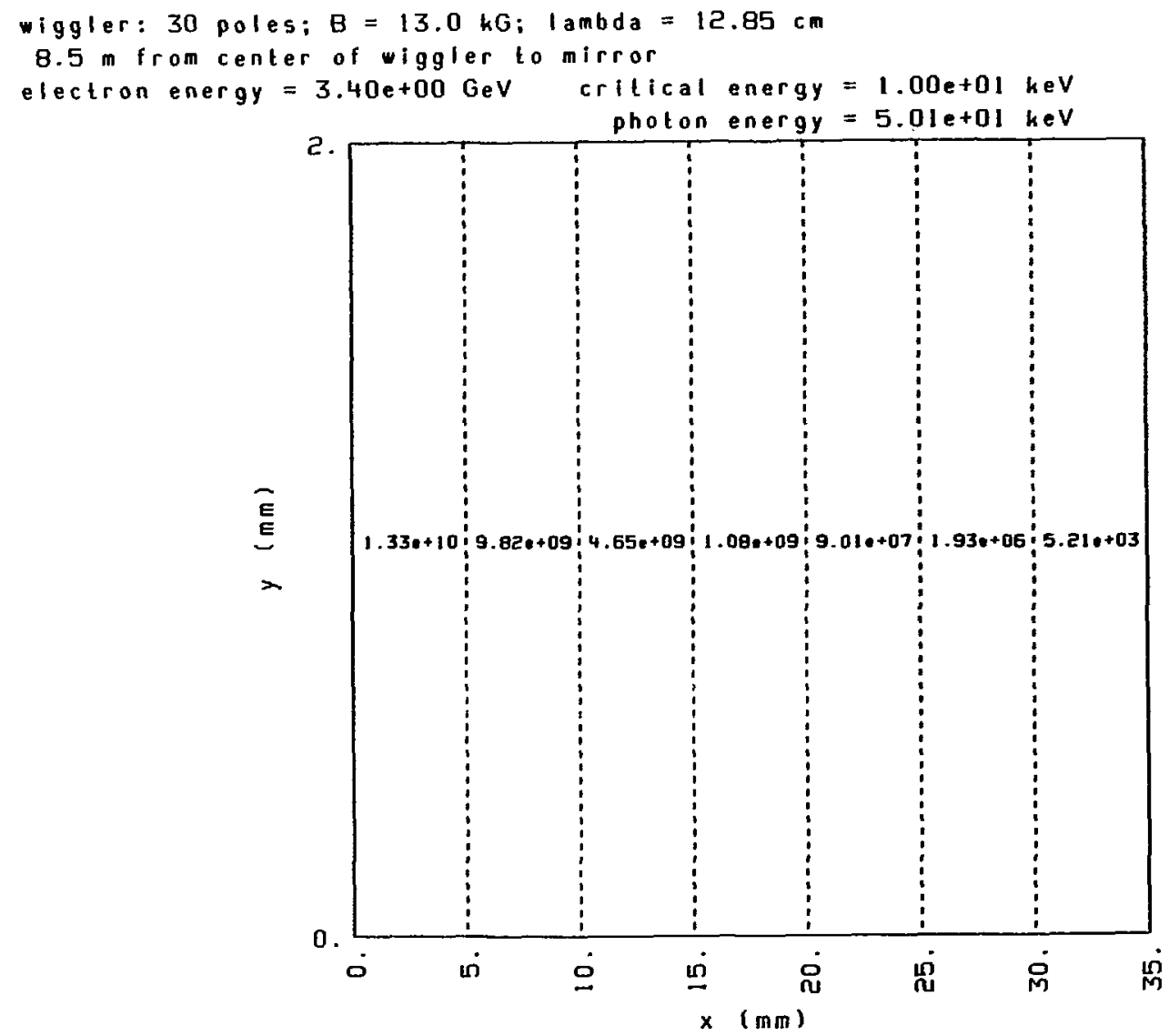

parallel

polarization

photons/sec/mA in $0.1 \%$ bandwidth 
Table C9.1.

wiggler: 30 poles; $B=13.0 \mathrm{kG} ; 1 \mathrm{lambda}=12.85 \mathrm{~cm}$ $8.5 \mathrm{~m}$ from center of wiggler to mirror

electron energy $=3.40 \mathrm{e}+00 \mathrm{GeV}$ critical energy $=1.00 \mathrm{e}+01 \mathrm{keV}$ photon energy $=2.00 \mathrm{e}-02 \mathrm{keV}$

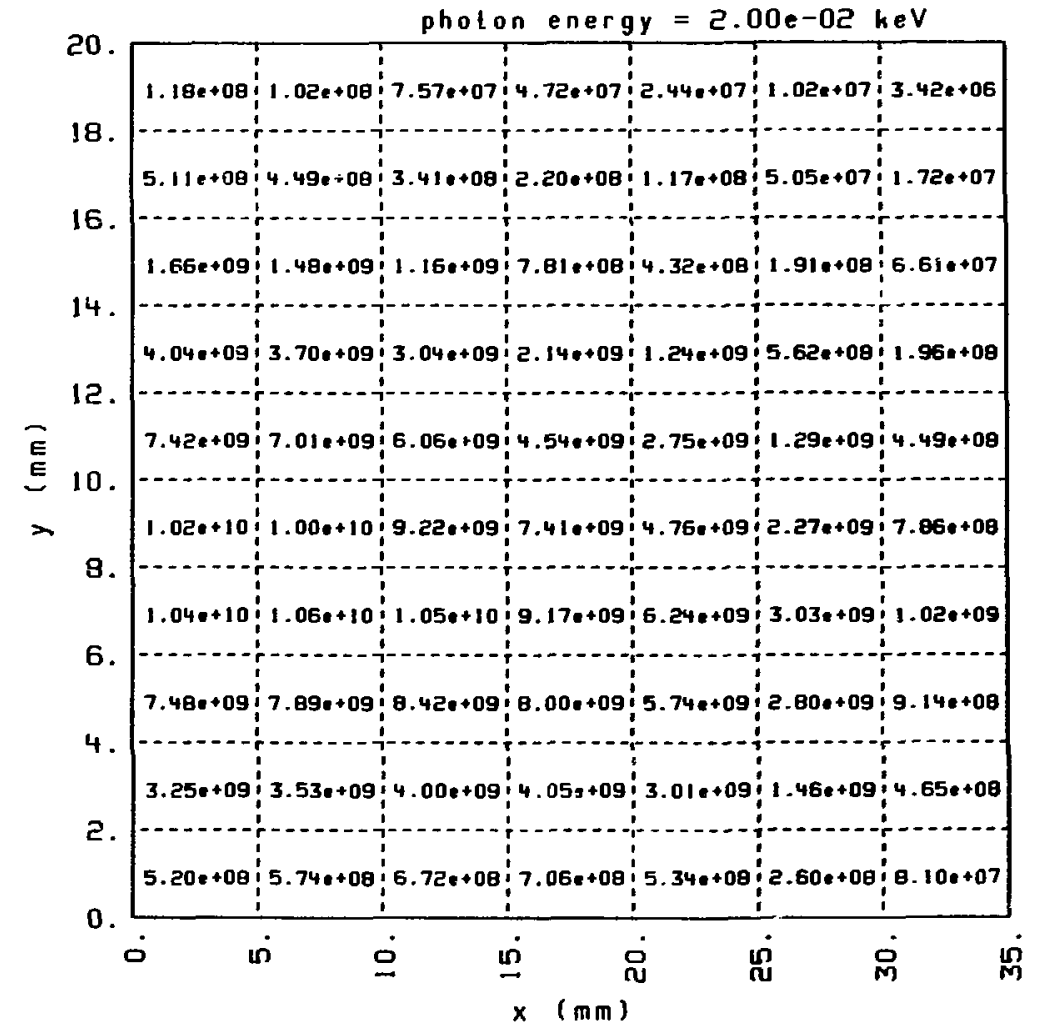

perpendicular polarization

photons/sec/mA in $0.1 \%$ bandwidth 
Table C9.2.

wiggler: 30 poles: $B=13.0 \mathrm{kG} ; 1 \mathrm{ambda}=12.85 \mathrm{~cm}$

$8.5 \mathrm{~m}$ from center of wiggler to mirror

electron energy $=3.40 \mathrm{e}+00 \mathrm{GeV}$ critical energy $=1.00 \mathrm{e}+01 \mathrm{keV}$ photon energy $=1.00 \mathrm{e}-01 \mathrm{keV}$

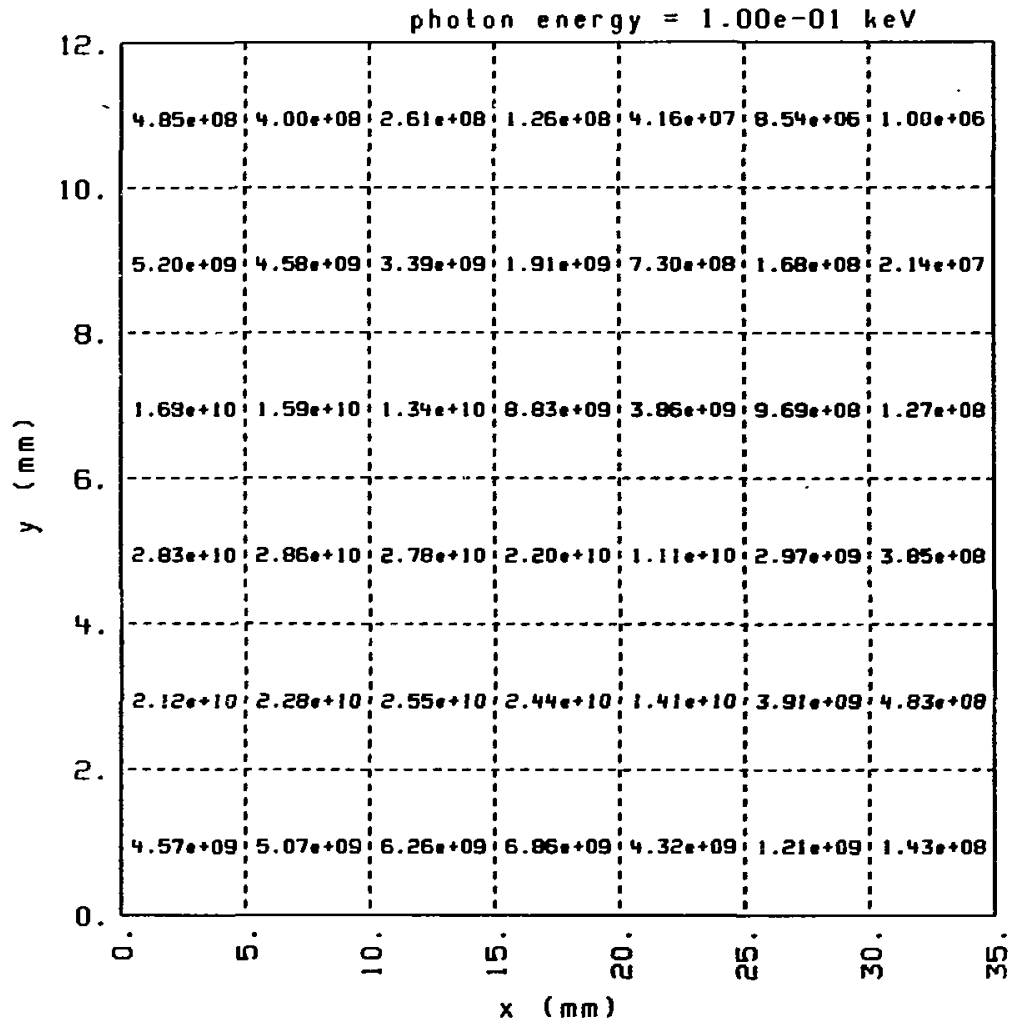

perpendicular polarization 
Table C9.3.

wiggler: 30 poles; $B=13.0 \mathrm{hG} ; \mathrm{lambda}=12.85 \mathrm{~cm}$ $8.5 \mathrm{~m}$ from center of wiggler to mirror

electron energy $=3.40 \mathrm{e}+00 \mathrm{GeV}$ critical energy $=1.00 \mathrm{e}+01 \mathrm{heV}$

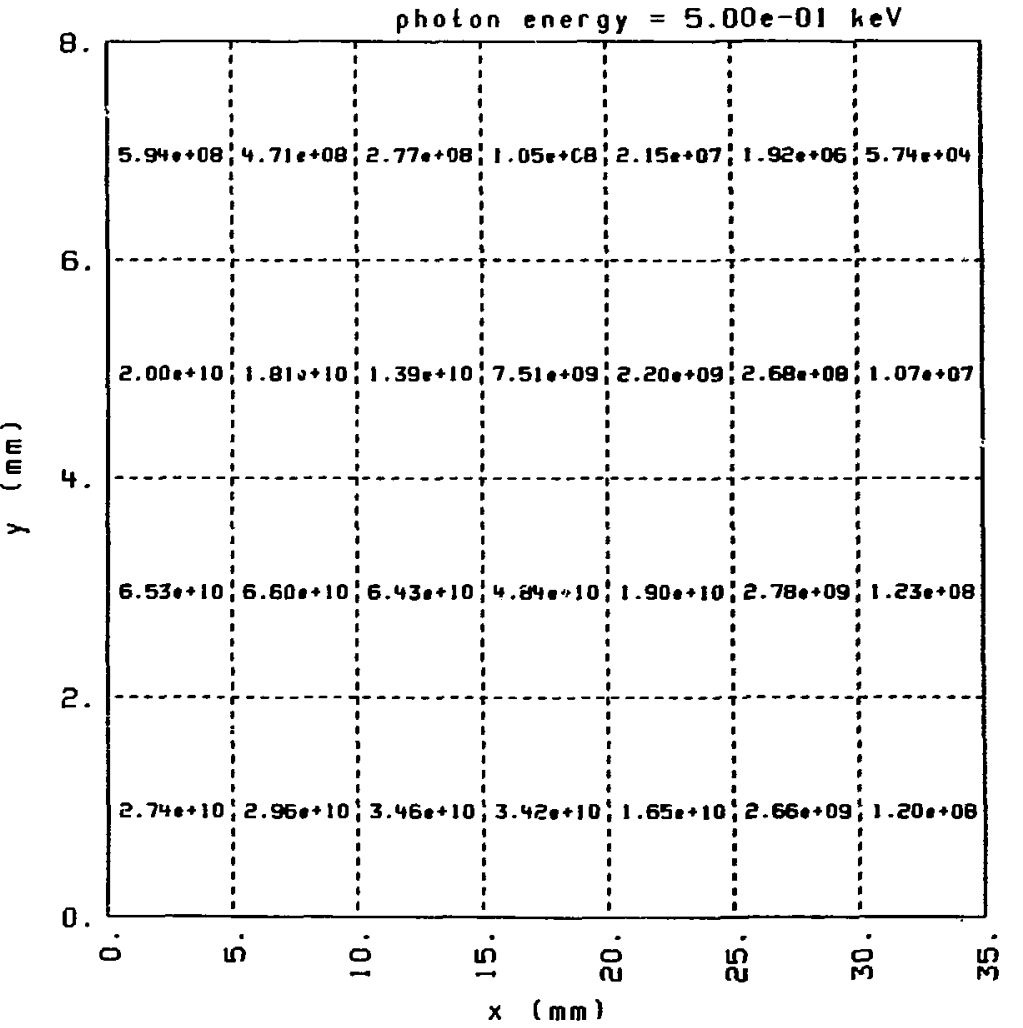

perpendicular

polarization

photons/sec/mA in $0.1 z$ bandwidth 
Table 69.4 .

wiggler: 30 poles; $B=13.0 \mathrm{kG} ; 1$ ambda $=12.85 \mathrm{~cm}$

8.5 m from center of wiggler to mirror

electron energy $=3.40 e+00 \mathrm{GeV}$

critical energy $=1.00 e+01 \mathrm{keV}$

photon energy $=1.00 e+00 \mathrm{keV}$

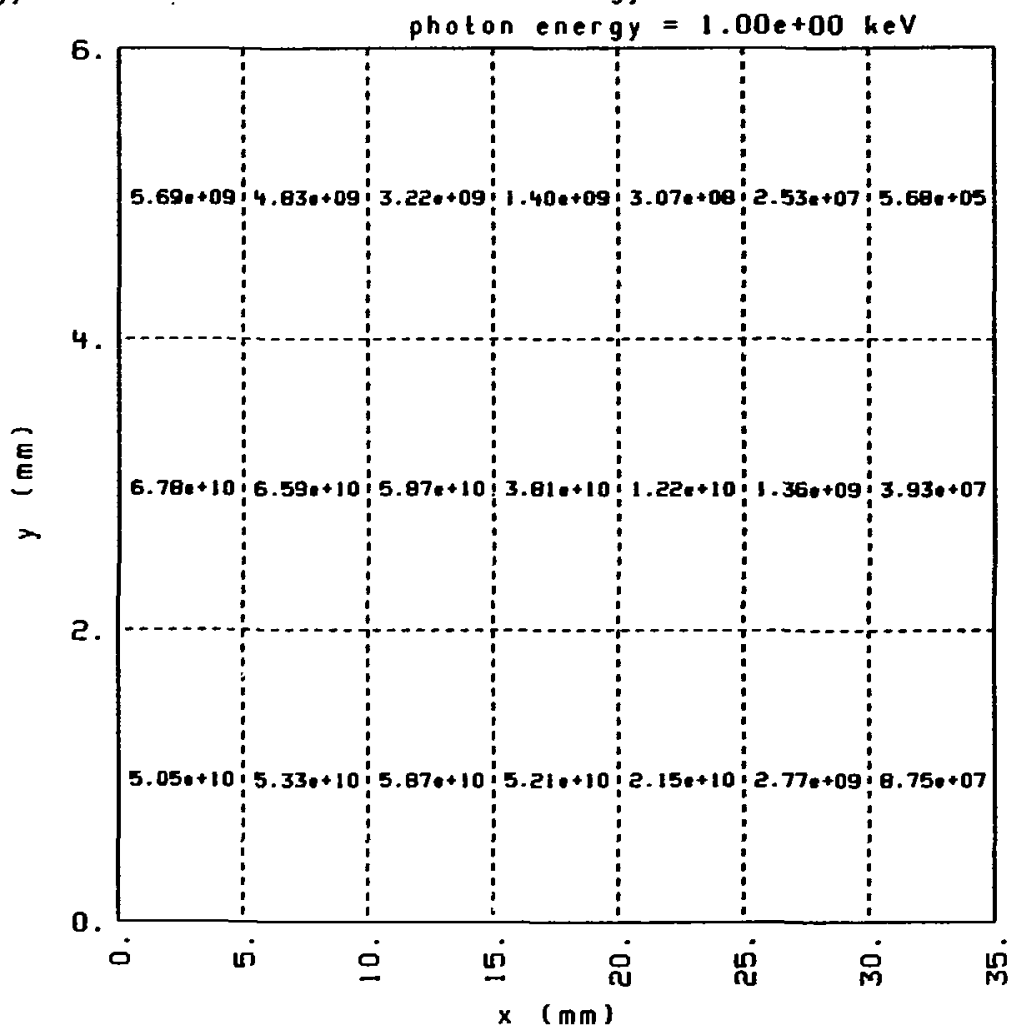

perpendicular polarization 
Table C9.5.

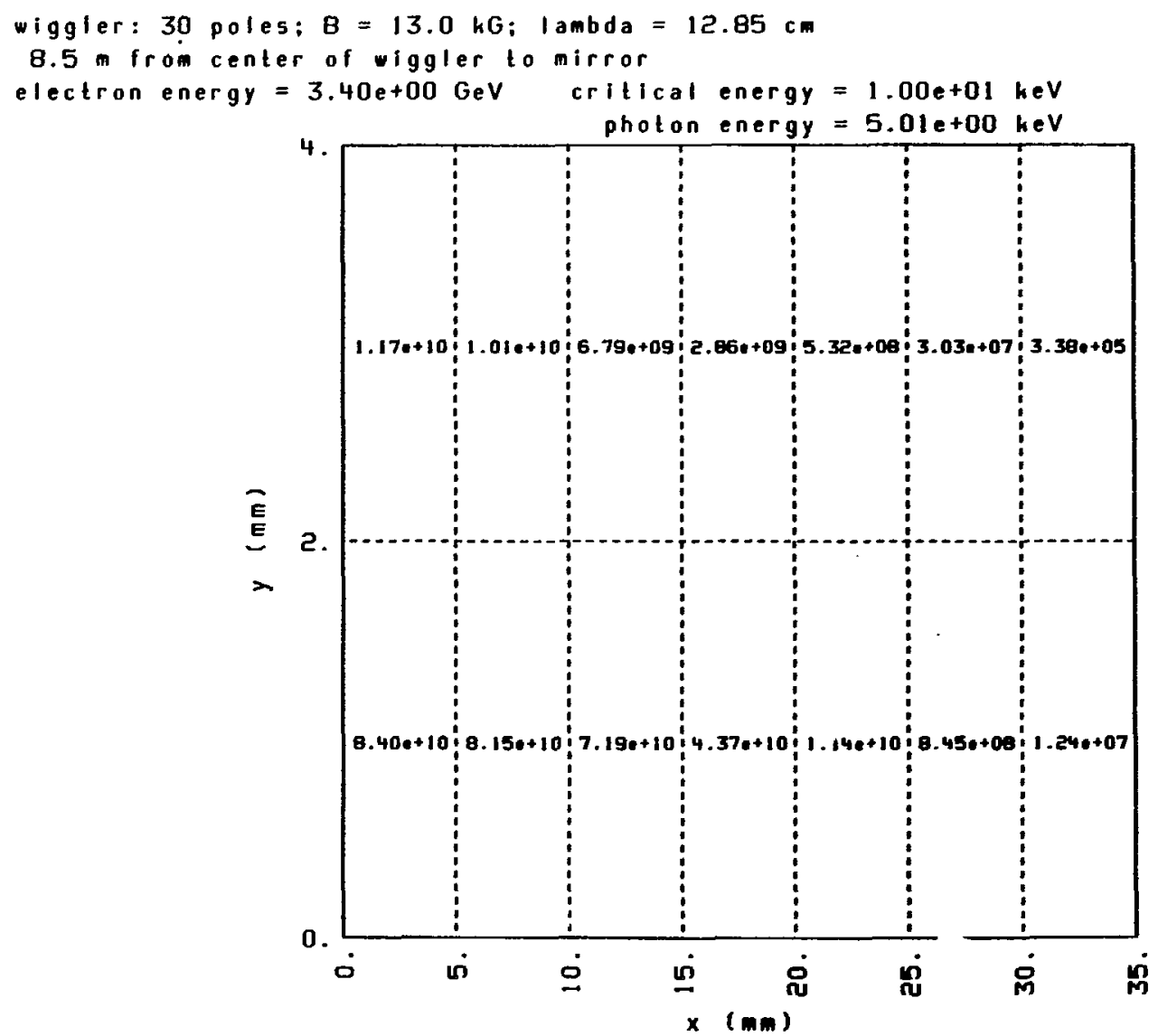

perpendicular polarization 
Table C9.6.

wiggler: 30 poles; $B=13.0 \mathrm{kG} ; \operatorname{lambda}=12.85 \mathrm{~cm}$

$8.5 \mathrm{~m}$ from center of wiggler to misror

electron energy $=3.40 e+00 \mathrm{GeV}$ critical energy $=1.00+01$ heV

photon energy $=1.00 \mathrm{de}+0 \mathrm{keV}$

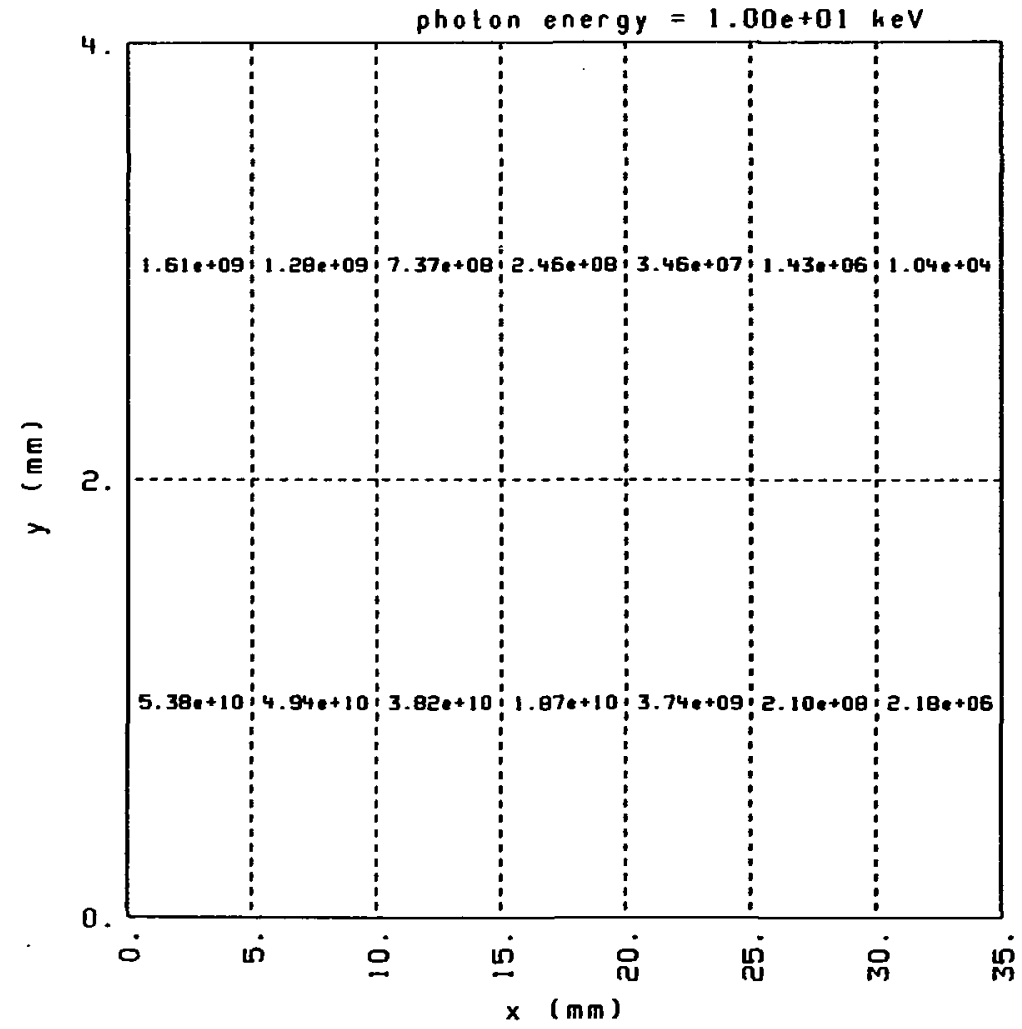

perpendicular

polarization

photons/sec/mA in 0.17 bandwidth 
Table C9.7.

wiggler: 30 poles: $B=13.0 \mathrm{kG} ; 1 \mathrm{Jmbda}=12.85 \mathrm{~cm}$ $8.5 \mathrm{~m}$ from center of wiggler : a mirror electron energy $=3.40 \mathrm{e}+00 \mathrm{GeV}$ critical energy $=1.00 \mathrm{e}+01 \mathrm{heV}$ photon energy $=5.01 \mathrm{e}+01 \mathrm{heV}$

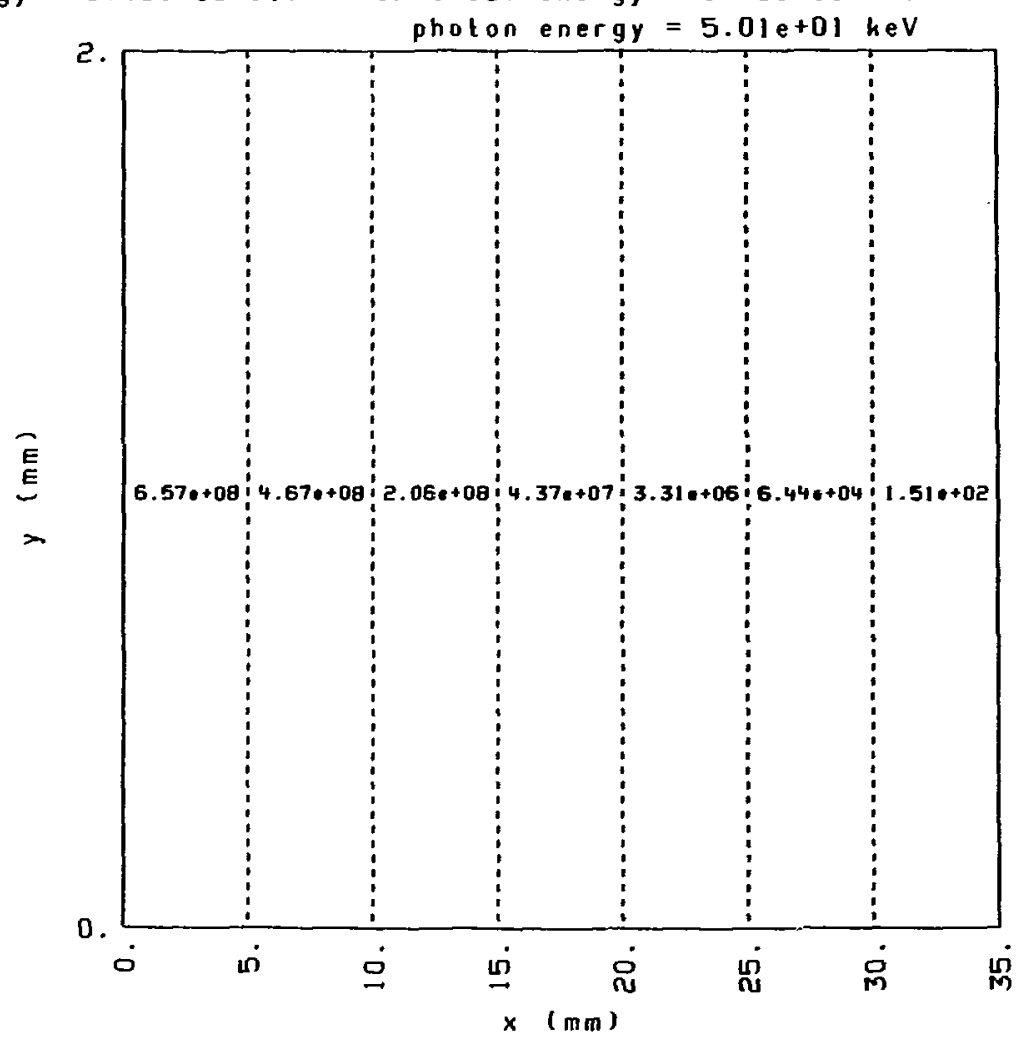

perpendicular

polarization

photons/sec/mA in $0.1 \%$ bandwidth 